$17-2.105 \times 2$ - Int

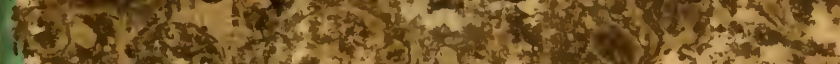
the

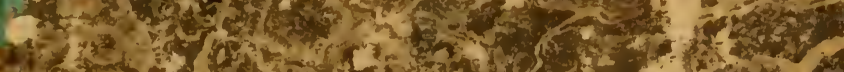

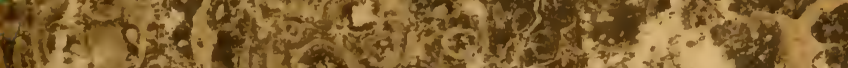
1.

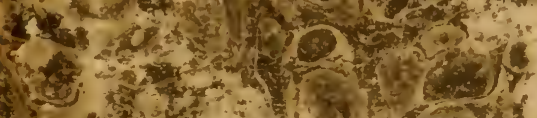

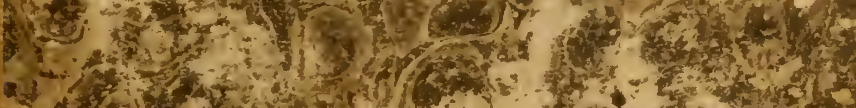

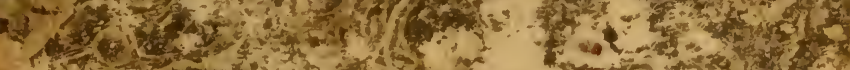

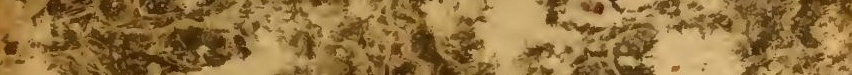

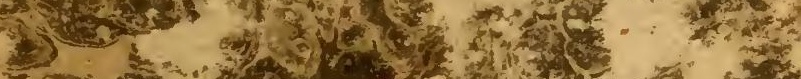

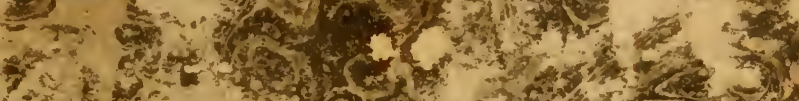

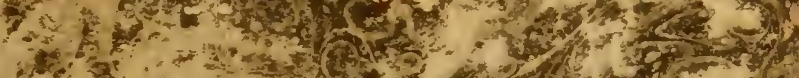

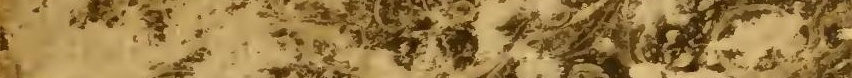
3 (3)

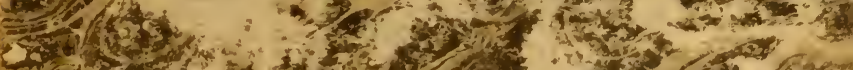
(1)

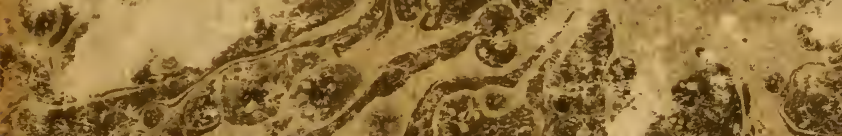

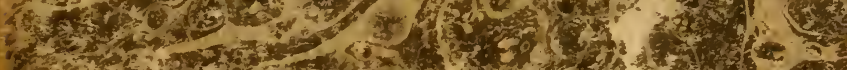

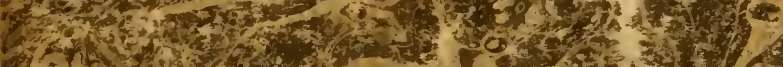
(3)

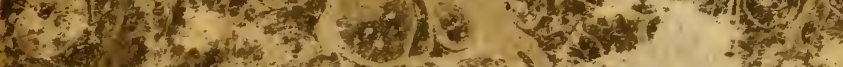

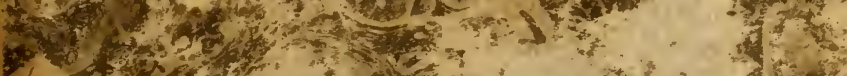

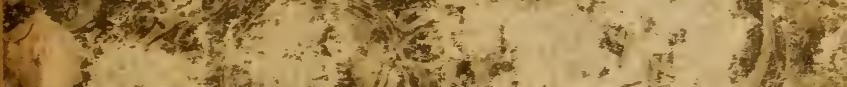

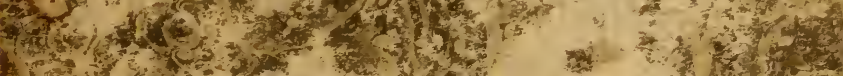

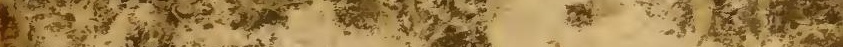

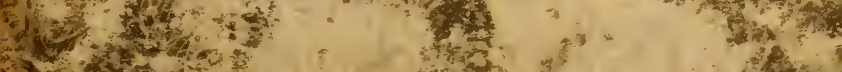

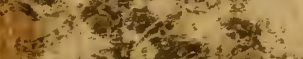

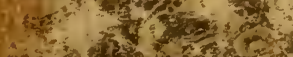

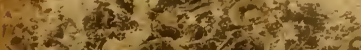

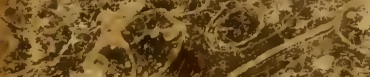
n. * ${ }^{2}$. the

ind

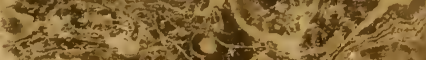

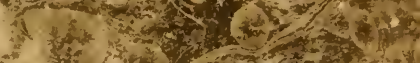




D ie

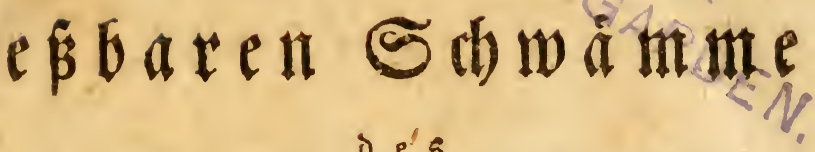

D $e^{\prime} s$

Defterreidifdet Maiferifaateg.

$\mathscr{B} \mathfrak{d} \mathfrak{n}$

ReDold Trattinnia.

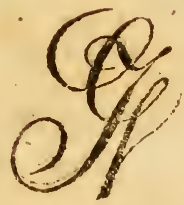

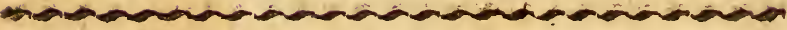

殁ien und irieft,

in Beiftingers Buकbanolung, $880 g$. 


\section{$\checkmark T 7$ \\ C. 6}

4113 il

Errando discimus.

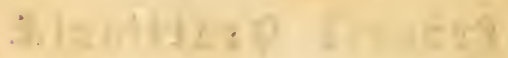

11

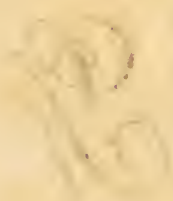

$1+2$ \& 1

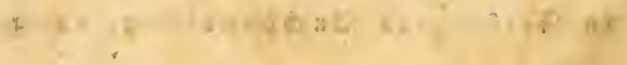


C c incm

\title{
geliebteiten Baterlande,
}

d e m

\section{glúcéliteden 22aterlande}

\author{
fo nieler
}

thatigar und tugenobafter $\mathfrak{B}$ ưger,

to großer und ausgezcidneter Bielef̧rten uno Siinitler,

To erfiabencr und unubertrefflicher Sclden,

\section{Der Stiege deg AdelB,}

Der Scimath

Der heiligften Religion uno Det reinffen Sittlidpert,

\section{$\mathcal{D} \in \mathfrak{m} \mathfrak{S} i \mathfrak{t} \mathfrak{e}$}

ber gerechteften, weifeften und beften

\section{$\mathfrak{M} \mathfrak{D} \mathfrak{n} \mathfrak{a} \mathfrak{l} \mathfrak{d} \mathfrak{e} \mathfrak{n}$}

miomet Diefe Edrrift,

fo unfedeutend fie allti immerfin penn mag,

a $\backslash \mathfrak{s}$

ein geringes Meremabl

feiner feurigften Riebe, feiner unbegränten Berehrung. und feiner wabrbafteften 2tmbänglidfét aus unbefangener Esele uno rauterftem 2untriebe

Der Berfaffer. 
$45 x^{2}+5020$

molth yis

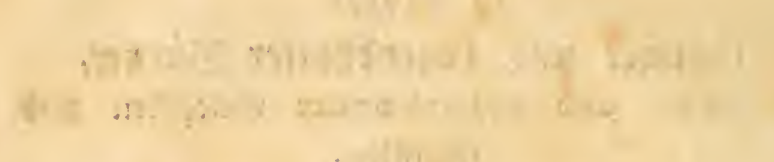

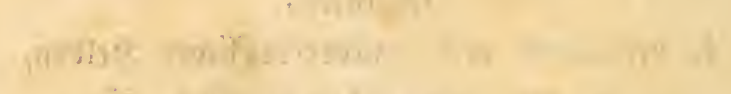

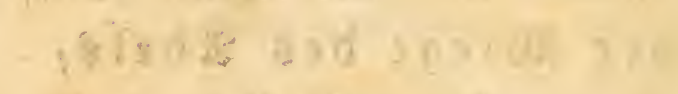

dhrtiaz

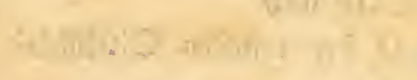

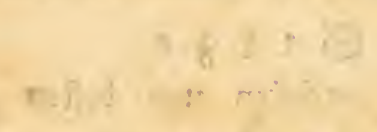

$11: 62502+40$

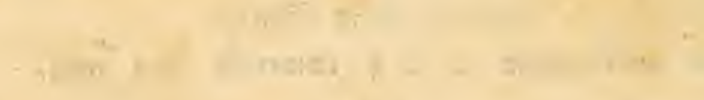

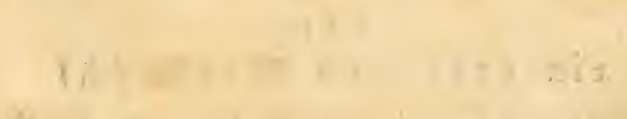

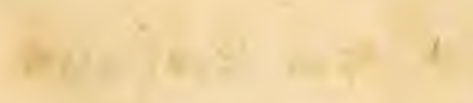




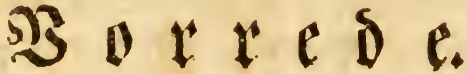

93

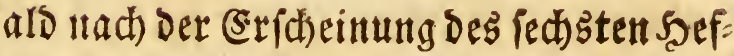
teg bon meittem gróf̈eren Schwammmerke,

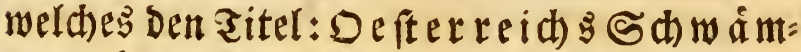

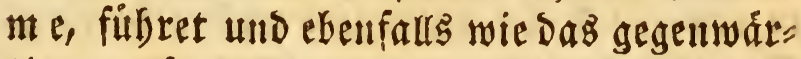
tige auf Roften Der jofeqlo seiftinger:

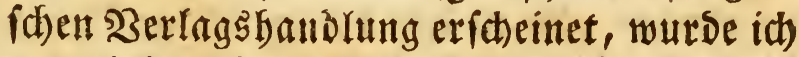
bon einigen fefre anfebnfichen Mántern auf eine nid)t weniger bumane als nachbrücflis che $\mathfrak{S}$ Beife aufgefordet, ein eigene von Den (Siftíd)wainmen Des Baterlandes zu fidreiben. Meine tiefe Berebrung für bies fe Sarren, Daz Éfrenvolle ibres Zutrauens und meine warme Theilnabme an Den Drang: falen der leidenden Menifhbeit und vorzůg. (ich) meiner biedern und guten Mitbürger lies

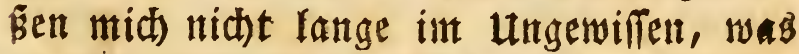
ich) thin follte. (Es wurde mir zwar fober, 
VI

Jild foltete mich fo manthes nicht unbeben tende $D$ pfer $\Im$ dh muste Daruber cinen aro= Ben Sbeil neiner angefangenen und für mid) meit vortbeilbafteren Irbeiten frad) liegen Inffen, id) muste ben 2 Bunichen und Furbes rungen meiner Freunde auf die Dauer dies

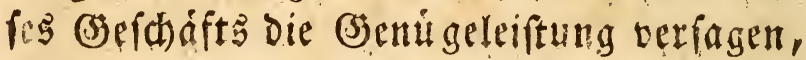
iof) mufte meinen Thesaurus botanicus in Det Fortiebung unterbreden u. f. w. Dod) mas fur ein Spfer fónnte auch einem gutgefinn ten etaatshurger zu thelter fenn, menn es barauf anfommt, Inglictśfalle ju vorbúten und Dem Baterlande felbit cinen reellen Dien ft zu erweifen? sch entichlof mich alfo mein Móglidhites zu thun und mein @chifflein fott zu machen um in Den sit erarifien Dcean binausulegelt und Dann, nit edjeken reid) belaten, in mein licbes 2 aterlerd mies Der zuricfuferben. stber die gide: gerade bon (siftichwómmen zu icherben, wollte mir nid) bebagen! "sd) legte meinen (sion. nern Die (Srunde bor, Die mid) fectimmtell, liber dif eśbaren Echwamme abzubandeln

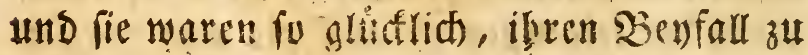
erbalten. Ins arsentlide viefer Grinde

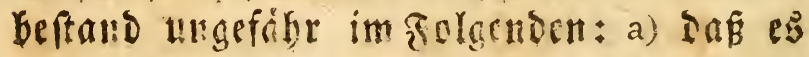

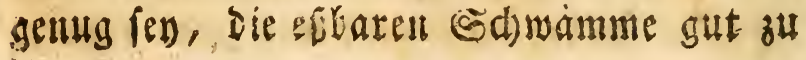


Fennen um fich vor ben giftigen zu bủten, weil bor ber Sand alle iene, dic bier micht mit aufgenobmen morden, fur forblich uno giftig zu balten waren; und bas b, Die Zabl ber eigentlid)en (3iftichwainme no(b) nidht

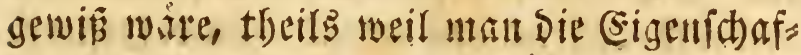
tell fo vieler Sumbert Sd)wámme nod) gaL nicht beobaditet bat, theils weil jelbit uber Die als giftig berufenen bie Neinungen ber Gad) fundigen uod fifr getheilt, und wirk = lich viele (Sirube vorbanden find, ifre $\mathfrak{a b}=$ folute $G$ d)ålid) Eeit zu bezmeifelu, wie man Diefes aus Dent, was am Ende nteiner Einleis tung gefagt wird, zแt (Senuge erieben Eam!

शRan bat die 2mitalten getrofien, Daf aud) bon Diefen eŝbaren Sdwåmmen eine zwenfache 2luğgabe, nåfnlich : Die eine mit Den Sadbofiguren und bie andere mit illumis nirten Rupfern feilgebotben werden Eonnte. Da aber in Der gegenwårtigen Sammlung e $i=$ nige Arten wieder aufgenofmen werden mus= ten, Die in Dem ålteren Sdwantmwerfe id)on abgeliefert waren; fo bat ber berr $\mathfrak{\text { Berle }}$ ger mit meinem Einberfitånonis befdlo loen, fiur Diejenigen Abnefmer des Sabinets (Der 33adigfiguren) Dielelken Strifeaug diefer ges 
VIII

genwårtigen હollection zurůåunebmen uns vom Preife abjuzieben, weid)e bereits in Deme nucologifden Sahinete Der Defterrechifden Sd)wámme abgeliefert und gefertiget wor: Den. Sn J̧infunft follet aber in Dem nunmefo wieder fort 3 ufesenden mucol. Eab. D. Defterr. Sd)w. Eeitte von Denen 2trten wieder ericheis nen, weld)e in Der gegenwårtigen Sammlung Det eşbaren Sdwámme enthalten finto.

Sein $\mathfrak{Z}$ ufteag bezielte cin $\mathfrak{B}$ ere, weld es einerfeits Durd) tribiale Sad)enntnifie und populåren Bortrag zur praftifchen 2nmen= oung tauglid); aber aud) zugleid fo einge

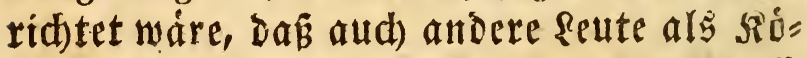
d) und Marttridter Dariul ibr Sntereffe finden Durften.

Dicfer 2 blicht bemilfte ich midh, fo gut

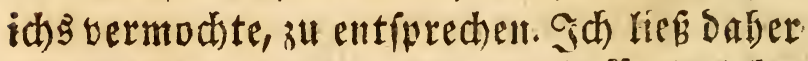
alleg dasientige weg, was nur allein firt (sel chrs. te von Profefition verbaulich $3 \mathfrak{u}$ fenn foten. S23as abor wefentlid) zur Renntniés ser Sadse geboret, und follte es aud nod fo neu unt noch io ernthafe fentl, sas fuchte id) alles int bundigiten Zufammenbange zu geben. F̧u

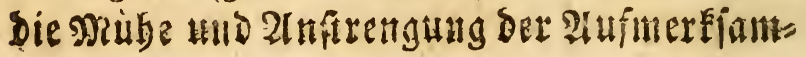


feit, Die bielleid)t manchent meiner Refer siefe secture foiten mag, fudte ich ifn burd) ei= ne gefállige Sareibart, Durdh pingentengte, vielleid)t nicht ganz unwirffame (Evifosen, Durch) originelle2nficten berDinne und surd) fo mandhes Blumd)en Der Rederunte (d)ad" Iog zu balten. Ja) boffe nofimlid) Dabur(t) zut bewirfel, Das ber zeritreung vorgebeugt werde und das man Das Sroctte und das Ernfthaftere um fo biel aufmerffamer ftudi= ren werde, da id) meinen (S)áten bas (serid)

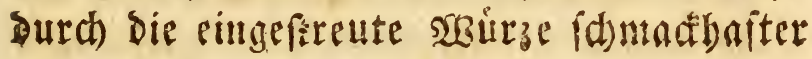
zu machen getrachtet babe.

Die cinleitung ift erit und $g a n z \mathfrak{b o l}$. fenbetem SBerfé gefd)rieben worden; und Da id) Dag Errando discimus vorjigfiab) auf mich felbit anmende; fo bitte id, ausiener bie $\mathfrak{F e}=$ richtigung zu fobpfen, went etwa in Dem Werfe ferbit ein wiberfpruch auffopen foilte.

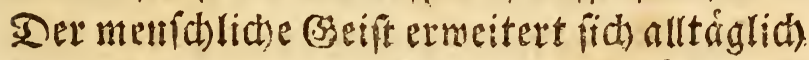

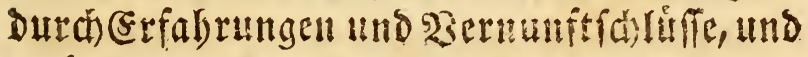
ro anderte (id) auch in bent meinigen man=

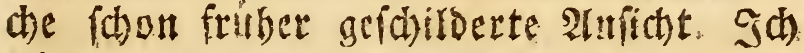
batte now febr biel zu fagen gehabt, was

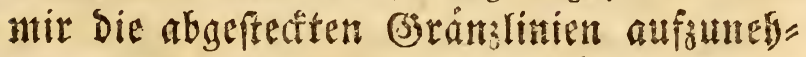

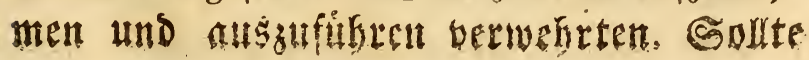


X

ich aber erfabren, Dak man Diefes mit aufriebenbeit aufgenobmen und nod) mebr uber diefe Materie zu lefen muniche; fo founte id nich wolgl einft nod) entichlinfell, Den $\mathfrak{B u n f}$ den meiner Freunde su begegnen, ifuen uber Die Ratur Der Samainme et= was febr 2 (usfitgrlides und ein mit jablecis chen Belegen aller ant terfebenes Sand buch, ou riefern.

WSien Den 6. Dešmber 1808 . 


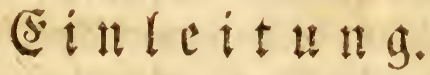

unom

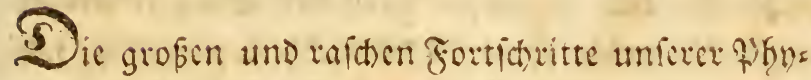

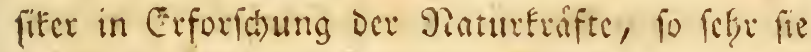
aud) immer beyfall und Ereftaunen verdienen mos gen, fie begrânzen fich nod inmer mit Dom 4 m fange des giaturreides der anorganiidian Sioracr,

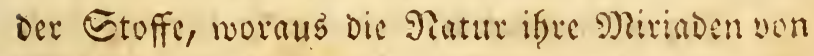
230 cien comptruit, und in die fie, nath gecnoigs

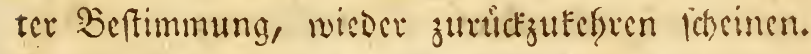
Wangt es aud mandhmal cin füfhner bicift, Die Befrche Der verganifden פiature entziffern zu wol= Ien; fo zeigt fich nur zu bald oce große atbitani

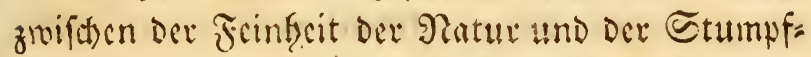

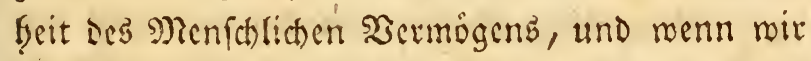
eine Zeitlang mitteidig zugeferfen, wie er dic $2 \mathfrak{B i t}$ fungen Der unż gånglití unbefannten Rebenstraft

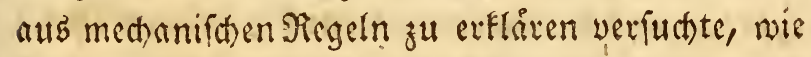
er Da Der Ratur, von einfeitigen beobad)tungen

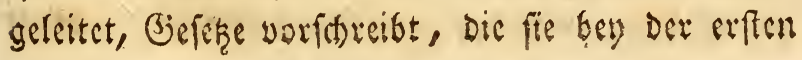




\section{XII}

Untcrfud)ung ourd Ungefiorfan verfpottet; fo exs rothen wir vor Der Sdhande, die unfere Fitelfeit auf eirmal von bem bod)fen (Sipfel des Stolzes.

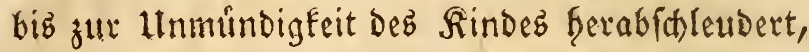

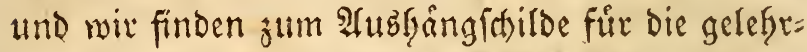
ten Shirngefpinfte Diefer medsanifaen. Pfy fiologen eine Devife, Die allen zufunftigen Gopfiften Jur Warnung Dienen follte und die uns in vier

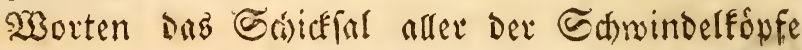

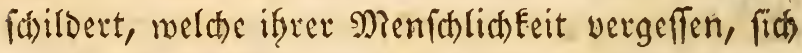
in Die fyofen Regionen ber Feffellofen Beifter ver= fteigen, uno Galb fwottelno, farl6 bednuerno rufen wiv ifnen nact):

\section{Icarus Icarias nomine fecit aquas!}

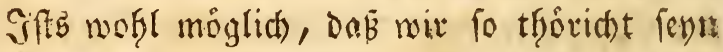
Fơnten ung cinzubilden, thum uno Sebengkenft Bienugthutng uno भaf: (a)luffe zu erfalten, wafgrend noir nod feiner im Stande find, aud) nut die innere Natur ber Me: Infle, ocr Ealze, Deb Feucre, der Ervftallifation, ber demifden Sermandtid)aft u. D. gl. zu exfla: ven? Mit welden Miafinen wurben wir woht ben Mann bezcidnnen, ber es wagen mollte, vielleidht von ber bloffen Arnalogie gelcitet, die innere s.in = rict)tung und Die Raturprodufte Der andern $\mathfrak{B B}_{\mathrm{B}} \mathrm{lt}=$ Forper uns finnlich Darzuftellen? und Dennod ift Der Corung von Dex demifh = medtenifden 23 elt 


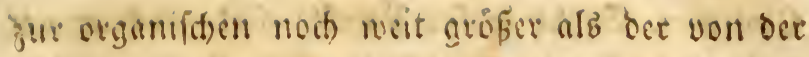
Eroe zu Den (jeftirnen! Bnar bat bie 9?atur, wern id) fo fagen barf, iffer Jiufe, voer vielmefre s(n= fangrountete, von weld)en fie auggebt, gemiffe Sirafte, geniffe Formen, gentife "Erfitseinungen

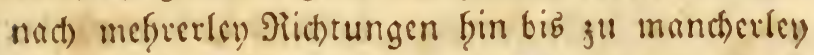
bald föfgeren bald niedrigeren Giaben ber $\mathfrak{S e l l f u m = ~}$ menfeit in $\mathfrak{B e r b i n d u n g ~ m i t ~ l e f ̧ r ~ b e t e r u g e n e n ~} \mathfrak{S e r}=$ foffeniffen zu werbeciten, uno fo ift zum (5r. Dab alleceinfact)fte If̧icr uno die unvollfommenfte $P$ flan= je jedes in feiner naturlicten Dronung auf einen fold)en Punft geftelfet, nelate cinander feffer nabe Fommen; aber won ien cinen aubentfaltet die S?a

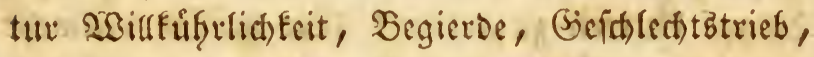
Şunger und Durft, Eelbffuct), Preigung uno Afbideu; von Dem andern fingegen nur Reizbar=

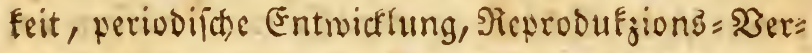
mógen, Dauerfiaftigfeit und 2Biederernecfung Der Fortpflangungz = Frfectionen; won Dem einen Die= fer Punfte fteigt fie finauf biz zum Diangutang, bis

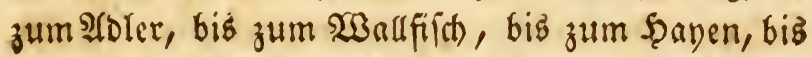
zur Sabilofrote, bis zur Riefenfalange, bis zum

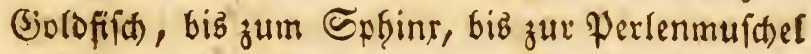
11. T. m.; von Dem andern erreidt fie die Şóhe Der Hedona, Dę̧ Hibiscus, Deş Antirrhinum, Des Hypericum, Der Musa, Der Thapsia, Des Cyno.

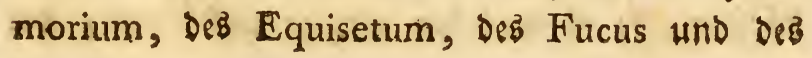




\section{XIV}

Clathrus. Selpoe biefer Punfte fino nod immer wefentlid) genug verfeticben, mie zmey Aleten cinet (5)attung (und in ber That fino fie and) nid)t an=

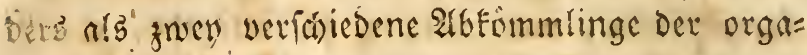
nifwen Gethopung!) fie mogen Demnad cinandet, nie inmer nate fommen; fo bleibt doch immer det

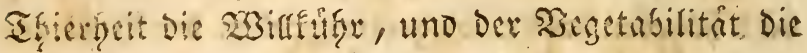

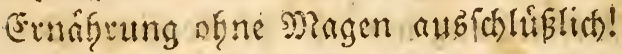

Bey Der Betractung Der organifitten $233=$

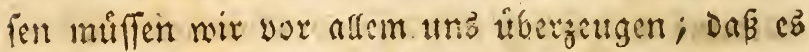
cin grober Fefsler foy, wie auf ciner feiter yon Dern Stufen Der gemifoten Fiorper zu ben nies brigften Giraden Der Ifficre und Der Pflangen Ginauf Flettern ju wollen! 23 ix múffen vielmefie guerft jenen Anfangşpunft auffuchen, von mel= bem die গatur ausging; als fie Den Plan ber organifach Sobopfung ergriff, wir múffen am allererften die cinfachern und, wenn id) fo fagen

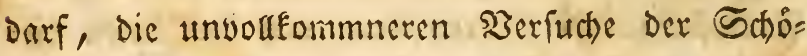
pfung ftubiren, auf welche in auffeigender Reife beffere, funftlichere, zufammengefestere folgen, uno bey welcinen fich Die Ratur nidht felten wies

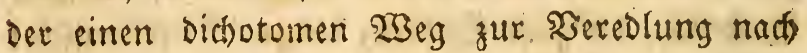
mancherley 3wedfen gefafint Gat! Und menn es ie gu Koffen ift, Das mir auf irgend einem Pfade in bie Beffeimniffe der Drgnnifation eindringen burften; fo if dies ber einzige unfeflelbare, Der. 
9iatur felbeft an beften angemeffene, weil fie im= mer nur vom Einfadien jum Zufammengefebten, vom-Nicbrigen zum Erffabenen, vom Sleinen jum Brofen fortjufdreiten wifeget. WBafferend Daf fie unz Dann bey Der unterften Stufe dicfer (bic=

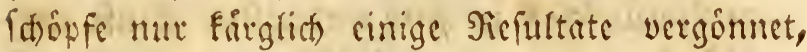
und irgend etwelcie unferer Zweifel entraitffelt, gelarigen nir defto geniffer, indem wir iffe Epu=

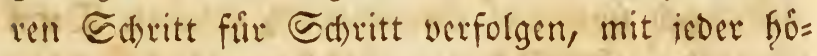
feren Etufe zu neuen uno fobgeren biefcimnif. fen und am (snde nofil felbft ju ben föchfent uno wichtigiten, die man nut irgend in Dem Sucte ber 9iatur zu finden fid fitmeid)en barf!

Schon bey Dem exfen blicke auf bie orgas nithe $23 \mathrm{clt}$, wir mogen fie won nas immer file. einer Ecite betracten, unterideiden wir jwo

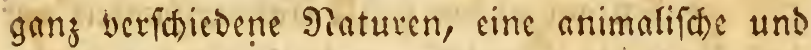
eine vegetabilifhe. Jene nicht etwa cin Gloker atbefommling Der lebteren, bat viclmefre iffen $\mathfrak{H}$ t: fprung, ifre niedrigfte Etufe gang nafe an bet Seite Der vegetabilifacen, und die animalitat ift bafer nicht etwa nur cine erfiofte und veredelte Negetrabilitåt. Die Negetabilitat mag noch to Johe Etufen Der Boufommenfeit uno Bered lung erreiden, fo wird fie fich boch niemals der Annimalität nåfern. Ulno wirflich lefret unz bie Ere

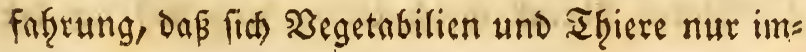




\section{XVI}

mer befto neiter yon cinander entfertien, iemefir fie

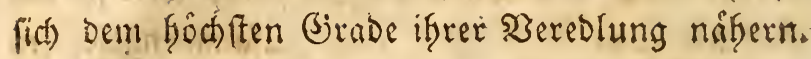
Ein Drangutang und cine Hedona find unliug= bar einander meit unaifnlicter, als ein Fadenbilz und cine Wolypengattung. Dennod hat bie Thies rifite Datur foton in ifree niebrigften Stufe eini= ge \$orzúge vor ber vegetabilifden, uno wir múf fen Dafier bey einer Naturalmetfode bet ganje

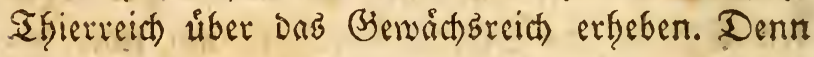

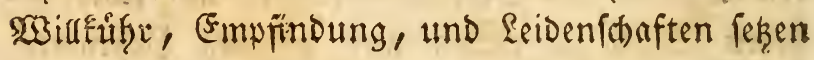
immerbin aud eine feinere und finftlictere Sorgas nifation voraub als bloß̧e Reizbarfeit! und wir fe= Fen liberdies, Dấ Die ertremen Stufen Der Ánima= litât weit erfgabner find, alz fich jene der 23 egetabi= litåt nur jemafilz erdenfen laffen!

Raffen mir nun Daz Tfierreich in ber Ferne, und wenden wir unfere ganze $\mathfrak{A} u f m e r f f a m f e i t ~ a u f$

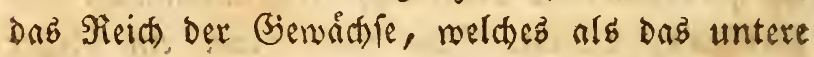
und einfadere uns aud eille frúfere Aerndte vont Refultaten veriprictit, Die Denn einft unfere Rad)=

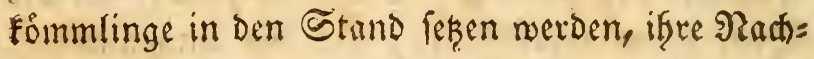
foridungen auth bis in bie erf̧abneren Bebietţe Der 2fnimalitä fortzulesen!

Dod) Eaum fiaben wir bie Edfmefle betrets

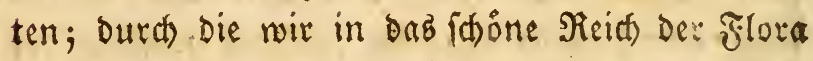
sintreffen; fo bemeiken wir auch fiton, dar fich Die शatur liberall gleich bleibe, und Dap fich bie 


\section{XVII}

SBegetubilien eben fo in whänerogamifale tho crys ptogamifac, wie die organifirten fiorper úbev Gaunt in Strimalien uno 3 egetatilien abtjeilen.

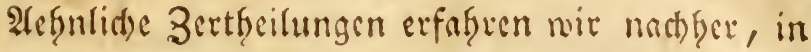
auffeigender Reife fowofl unter Den Phanerogas men wie unter oen Eryptogamen. Uno alle biele Ecitentfeile oder Etraflen auङ Dem Mrittelpuntte Der anfangenden 2iegetation find von folder $B_{e}=$ fdaffentert, Daß fie eben fo liber cinander gefegt werden fonnen wie Thierreid) und (bieneid)greid), ofne thbrigens einen eigentlid)en Bufanmentang und eine Siemeinichaft als Dic Der Negetabiliteit, Det Wgånerogamie u. T. w. z"l Gaben. Denn Die \$hainerogamen find allevingz coler als die Ciypto= gannen, Die Sompleten coler alö bie Serwortenen, Die Normalpflanjen eder als Die Nermanden

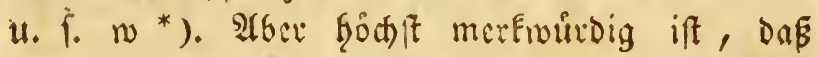
Die grokęn Malien Der naturlichen Familien des

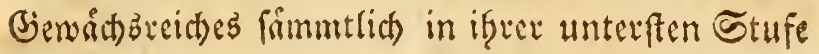

*) Эฺ besiebe mid bier aufmeinen Methodus naturalis plantarum, an beffen $\mathscr{A}$ (usfubrung it) fiton feit vilen Sabren arbeite, wodon aut) bereits im $\mathfrak{Y} 1802$ ein fefre undollems mener Crntwurf erfitienen ift, und welden id́) nun bald in einer gan: Deránderten B̧eitalt dell Freunden ber SotaniE vorlesen zu Eounen boffe, ungeadtet id fefor uberzengt bin, baf man now utenistid oiel zu verbeffern finden werde, ebe sin foldyes Werf als complet angefeljen werben fanu. SAbee eben diefe Mängel miffelt erft befaunt nerben, um iurd

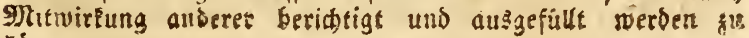
tonnen. 


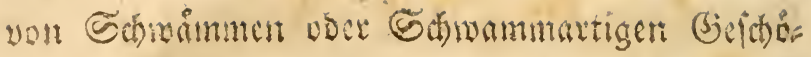
pFen beginnen; Dent Die Stermortenen Gaben z. 3. Cynomorium tutb Balanophora aufgumeijen, 10

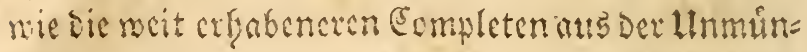

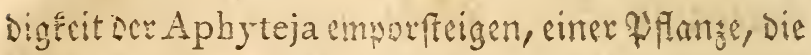
fo gan: Eromamm ift, Das fie fogar den (beruch uno (Befomak mit Dicje: Familic gemein Gat, Die naw iffer Scftalt ju Den Sntothecien, nad) Den Ne= ben= Dignenen zu Hydnum gezafilt merden müpte, Die aber Demod fo vorfferndig gebaut ift, baf fie Sielo, Brtumenthlitter und Ncctarien, Staub= - gefalfe unb gollen, Marben uno Frudtfnoten, entfaltet, fo wie fie aud am Ende cine grope Frudt, eine Deutliffe Bece bevworbringt!

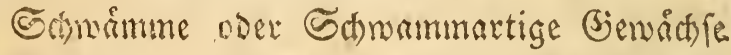
find es alfo, in welden die Ratur ifre fimpeln Sinfinge Det Sigetation unb vor Die Aligen ge= fteffet, in benen fie dic geringfte Eomylication

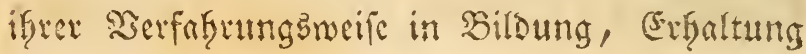

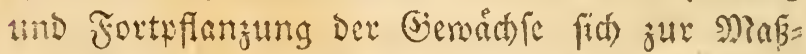
regel genommen, und in weldsen fie es unz fo= Dann an leibteften gemarbt bat, iften Gefgeima inffen nuf bic Epur zu fommen, und fowogl itber şegetation a!s Drganifation ůberf̧nupt ci= nige nd)! fo whinf(b)

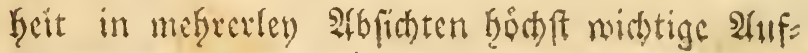
fdluffe zu exhalten. 


\section{XIX}

Sin ftrengen Sinne find ez zwate nidyt tie Ed)wamme felbit, fondern dic Sylfubartigen $20=$ getrbilien, dic ich für die sinfactfien tind Dem Sinfangstuntte ber Negetation am nádyiten an=

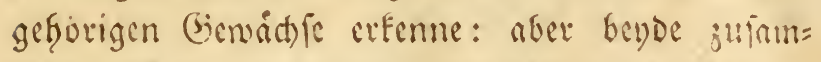
men mathen in meinex ?aturalmetfode cine be=

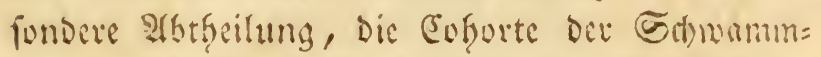
artigen (Fungosae) aug, und umfaffen zuram= mengenommen Die genje Aththeilung Der Perfoun= fiben Fungorum Gymnocarpium. Heberbick fiat es mit Den Syffusartigen (Sienádjen hod) ein be=

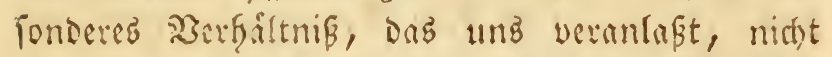
fie, fondern viclmefre die eigentlidyen Eabwan= me in Die Eateguric ber cinfactyen und uran=

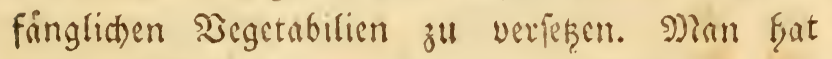
niffmlid) bereitz an mefreren Derfetten die (Ev faffrung gemadht, daßj fie unter gemiffen llu= ftânden, Die ifre weitere Entwieflung begünti= gen, fith in wirfliche Schwamme, in Theraepfio: ren, Boleten u. D. gl. wermandsln; tund man zrmeifelt alio mit vielem Birunde an Der Weecent= fich Éeit biefer Familie, die weelfeidst nictits ande=

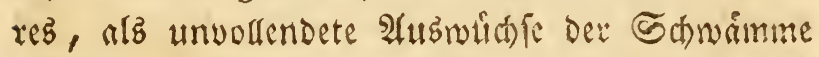
enthålt, die wegen Mangel des Sidts, Der Luft น. f. m. gefindert waren, ienes Drgnn jur Sesife zu bringen, Das bey ben Edwaimmen zugleits Gie Stelle Der Błtumen und Der Früdte vertritt, 


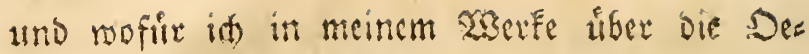

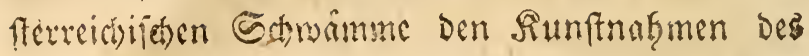
Jrudteriptis (Encarpium) feftgefest frabe. Dematium bombycinum unv Mesenterica argentea fint nat) Der Scobad)tung Des Palisot-deBeauvois (i. Annales du Mus. d'hist nat. Cah. 46. IV. Ainnée p. 334 et seqq.) uno fogar falon nac) Dex (Exfafrung Des Vaillant (5. Defl. Botanicon Parisiense p. 41.) nid)tz meiter ald Stu= fen eines unausgebiloeten Boletus, ja fie fonnen fogar in ciner gewiffen Lage zux Himantia mex= Den. Jah felbft Gabe Diefe ß̌cobachtung ofterz wie= Derfoblet, und id Gabe cin Eremplar yor mei= nen Sugen, in Dem man eb Deutlich fieft, wie Die fich Dureffruzenden Fenden dez Dematium fiá) gleioffam jufammenfricfer, um die sód)et eines Boletus zu billoen, melder nidjts meiter a!z Boletus (Poria) Fimbriatus Pers. iff. Rhizomorpha subcorticalis wito nat Palisot-deBeauvois (a. a. D. p. 338.) zu einem Boletus, und die Himantia domestica fabe id immer als

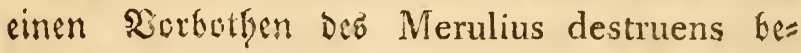
obachtet, fo Das ich fie fư nichts weiter als fư Dab Mycelium Deg lesteren erfennen fann. Racodium cellare entfteft in fefre feucten Rels lern und auf wollen alten 23 einfaffern, verwan= Delt fich noer in Dematium Bombycinum, menn 
eb tructner mitt. Racodium Corium frabe id

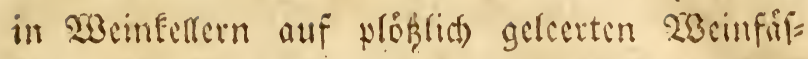
fern, vorghiglid, aber an ben untergelegten Sal= fen (Sattel) atts Dematium Bombycinum wer=

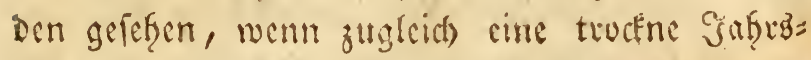

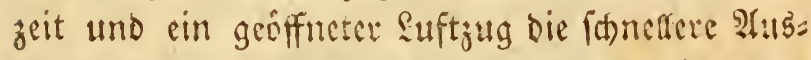
trodinung Des leştern benitéte, uno cine yor miv neuentocefte weise afut won Rhizomorp a,

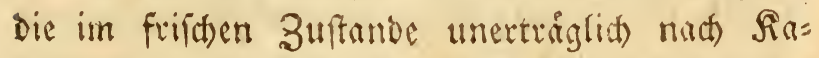
Benurin frinft, und in Brunnrofgren uno tiefen

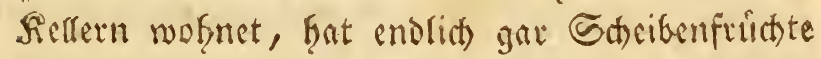
(Orbillae Achar:) getringen. Sit) babe fie cinfta meiten abgebildet, und ifir, ba fic eire gans netle Species iff, ben Nenfmen: Cornicularia subterranea beygelegt. Miefreere beypicle forinen woe

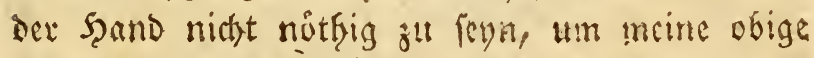
Affertionen zu befrafrigen und uns gegen bie

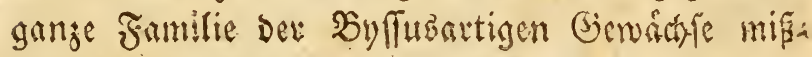
tranifh ou madten.

Son allen $\mathfrak{B e g e t a b i l i e n ~ f i s c i n e n ~ b e m n a c h ~ D i e ~}$ Sdymamme am beften gexignet fu feyn, uns in Die Bjeryeimniffe Der Segetation cinzufufrecn unts

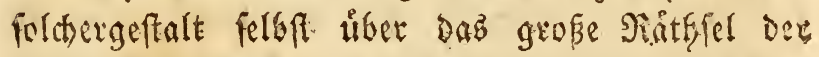

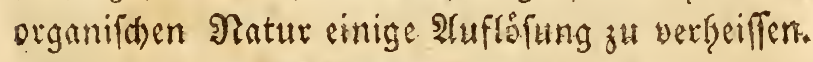
Ittein ez frópt uns bier eine Jrage auf, die notf̧wendig efer beantwortek weroen murs,

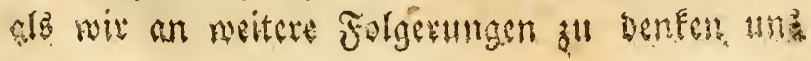




\section{XXII}

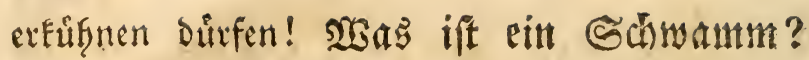
In Soigts Magazin Der Wfyfif und Faturge=

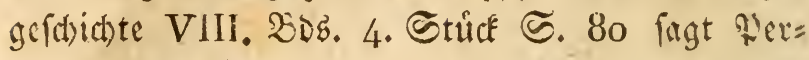
foon: Ein Sobwamm ift cine Pflanze, sie fich bloßs alb nadete FruEtififations= Theile Darfeclt; IInd in friner Synopsis fungorum edit. $1 \mathrm{ma}$. De= finet or 1 inn mit folgenden $2 \mathfrak{S o r t e n : ~ F u n g u s ~ e s t ~}$ vegetabile simplicissimum, solummodo partes fructificationis autpotius, dum ut plurimum jam maturescens invenitur, fructum sensu latiore sumtum, nudum exi,ibet. In beyben biefer Defi= nitionen feflt cin mefentidter SGsil, Der Gchwantm felbft, Denn die Frucht Des Schwammes ift eben fo nenig : baum. Beffer und wolftandiger ift bafer folgende Des sern. Dr Saterle (G.Deff. Commentar, z. D.

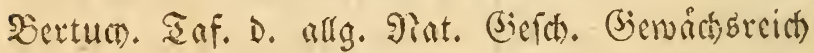

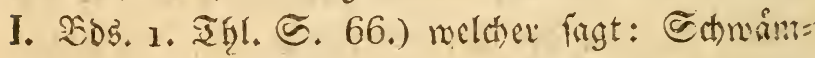

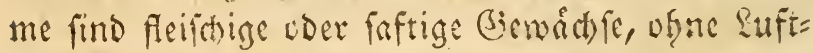
gefirbe uno Gamenlappen, fo wie ofgne laut

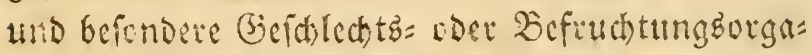
ne, Die ifre zur Fortpfingung Dienende brut

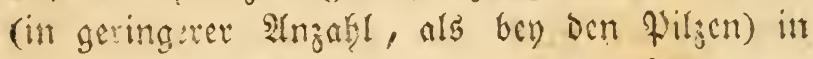
cinem frentiegenden fräutchen - Dem Edury (Hymenium) craengen. Itrein aud Dicfe Defint= tion Gat einen Fefles, id meyne ton ber su gropen 23 cithuftigfeit. Ianf id es magen, an iff 


\section{XXIII}

ter Stefle cine antocic neue gut werfuctsen; fo ift

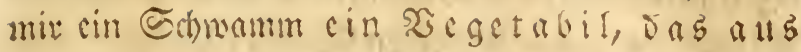

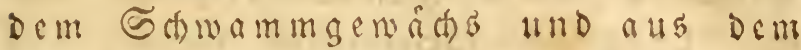

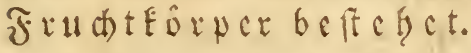

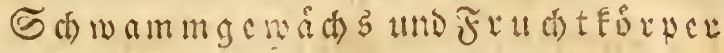
find aber zuely won mix neu cingefifflte fiunft termini, mowon eg alfo meine Pflidst ift, frict einte beftimmte (EvElárung mitjuthcilcn.

Unter $\widetilde{6}$ d) mihi, oder Carcithium Neckeri) verftefe id) ic= ne Siallertartig = faferichte Subftang, melda meis frenthcils mux untcrf̧alb ifices Etandortes auşge= goffen, bloß dic FunEtionen Des $\mathfrak{B}$ ad zind Der Ernáfung of verridten beftimmt ift.

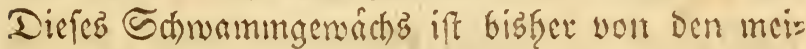

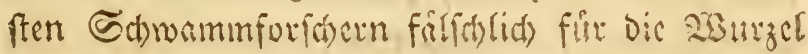

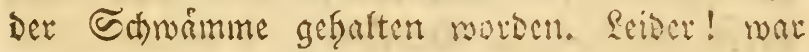
Diefer Jurthum die litfache, narum man ç bisz= ber faft ganglich vernaclalifigte, Da ç Doch ơt Unterfacioung Det Sleten eben fo refintlich und notfruendig ift, alb bey ben wirnerogemiflen

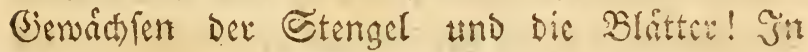
Dex Shat ift es aud weit mefreten sicuinocrum gen unterworfen, als Miandibe fïb) cinbilbet! (5) ift cinjaffrig, ober percnmirend, es liegt ent= weder ganz unter Der Erde, oder Hocrfrigt fei= nen Gtandort und biloet cinen Eshwammits 


\section{XXIV}

(Symphoresis), ein Biegenftucf yom $\mathrm{R}$ hizoma dee Cerualpflanzen, oder cinen $\mathfrak{S u l f t}$ (Volva), soer ein Mittelgenád (Scleromium) wie beis dem Bunderfotwamme u. f. m. Ifuch diftinguirt fid bag Mrycelium ourch fefte mannigfaltige Mlosififa: tionen bes (jerudbes, Der Farbe, Der Eonfiftenz,

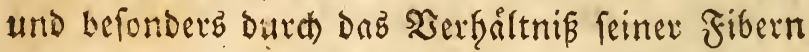
ju Der Malfe Der Baffertartigen Feuthtigkeit, $\mathfrak{B e r}$ bialtriffe, morauf es ganj befonders anfoimnt, ob bie Atet foneller voer langfamer vegetiren, anfeftn= liche ober nur Gagere Frudthorper Gervorbringen folle H. D. gl. शach meinem Sinne ift alfo gernor Das Mrycelium felbft der Gorinamm, wag fonft

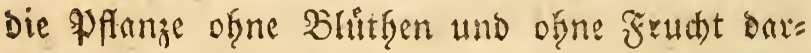

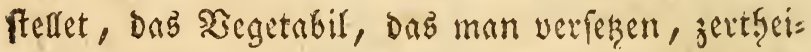
Ien und propfen fann, und welehes man witefich

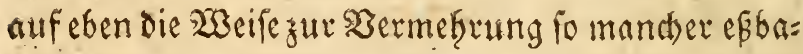
ren Sdnumme, bifonvers der Egampignons (Agaricus Pratella campestris $P_{\text {.) }}$ vernendet, Dab vft

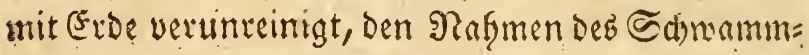
fteinç (piatra fongaja) fitferet, uno wodurct) Det Frenferr v. Jaquin Der jungere Den Boletus tube-

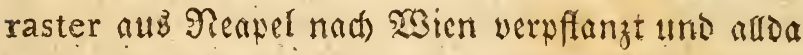
mie eine anocre Pflanze im Giewádabfante ers

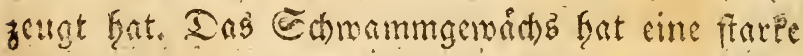
IInafogie mit geniffen Fleátengattungen, befonders mit ben Gafertartigen (Collema Ach. D. i. Dor ö. 
Interabtheilung Der (3)artung Parmelia!) und nod) mehr mit gemiffen Pfycren, oder 23 afferals gen, alş j. B. mit Ulva, B atrachospermum u. D. gl. Decen Eubftanj eben fo Gallertartig uno mit Fibern Durd)webt ift. Da nber die Flectiten bloß über Dim Etandorte uno dic Phycien blos un= ter bem saffer vegetiren, fo ift bey ben erferen fein Giund vorfianden, warum ifre Fruchtor: gane von Den vegetativen fo frare verfeficden feyn forten, nie die Fruhtorgane Der Edymanme. Dagegen fructificiren die phycien ganz unter Dem 2 Baffer, und wit fonmen ifinen defrwegen noch) weniger Şeterogenitåt zumuthen alz ben Flecteten, Da iffe functification nicht einmafil aus Dex s)?affe dez SEegetabils ficrvorbricht, fon= Dern nux eine Art von Geidnulft fervorbringt, ưber Die fich Dab (jemách Durd Prolifiention

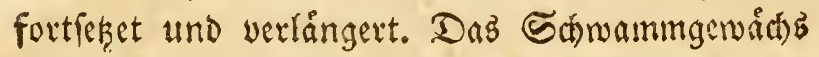

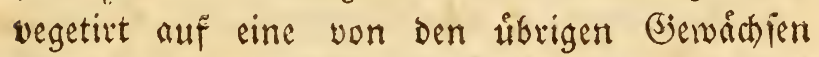

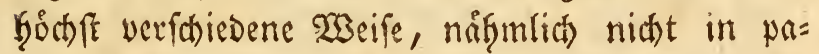
raleller Ridstung nach aufwärts, fondern viels mefri in Safangenlinien und fich ourd) freugen= Den Beftricfen. Die Fíbern Desfelben Durd)weben fich im Fortwad) fen, und Gåfen fith unter ges wiffen Himftinden wie cin bidter Wolfter an. Diefes Zufammendrángen vermef̧rt aber, bey fort wrifrend günfigen Hmftinden, Die Begetationb= 


\section{XXVI}

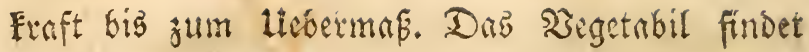

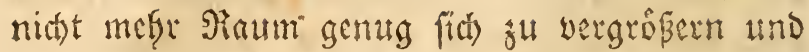
feine Nafirung fü fich felbft su verwenden. (5) fondert alfo, um fin gu erleid)tern, cinen sheil feimer Eubftanz aus finem Snnerften aus (uno

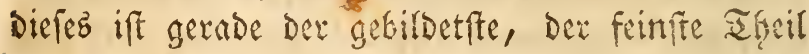

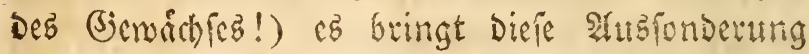
nn Die freve \&uft, afrwo cin neuer Sicis Dicform Drgan citte scronderte Siá)tung mittgeilet und eb Ju Denienigen Functionen beftimmet, Die nir Die

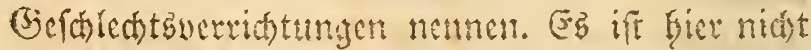
Der Drt, Diefe IGcoric nod weiter ins Detrí

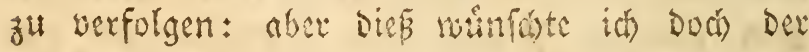

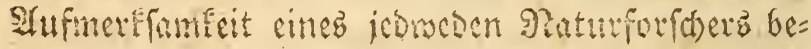

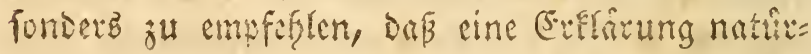
licher Erforeinungen in Da: organifhen 23 elt nie= mable fo wie der Giang cinet pendeluór aus Der Jick)anif, fondern cinzig aub Den yon Der Seand

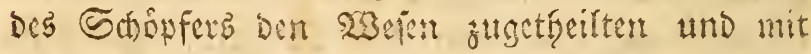

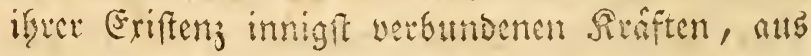
Eimnitung Dex fuffertichen Dinge tmb begen= wirfung ber RefengEnft erfert werden miffe, nichtauz der Attraction, Smprtctrobilitat, Somcre, Einficitat 4. F. n.

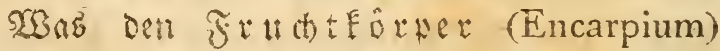
betrifft; fo Fabe id juar foron im Novifergetgen

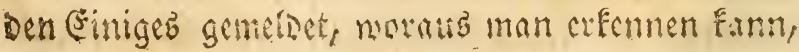


was id Sarunter werftefre. Aacin man witd es

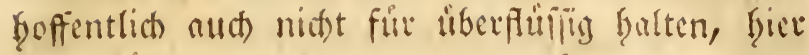
noch Einiges nachjutragen, was uber bicfen (bie= genftand nod) mefer licht ju verbeciten taagen Dirffe? Der Hrfprung Des Frudteforpers ift, wie nix foon miffen, aus Dex innerfen Eubftanj,

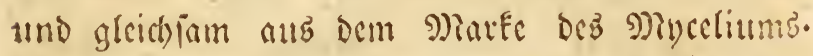
Da cr feinen Bunachs nicht unmittelfat von ausen, fondern aus Der fobon fomegenifiten

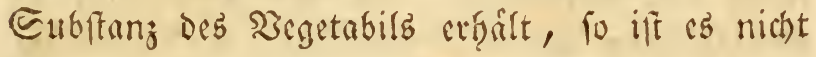
fdower oll begreifen, warum er immer biel com= pacter, feiner und reiner ift, alb Das 9lycelium.

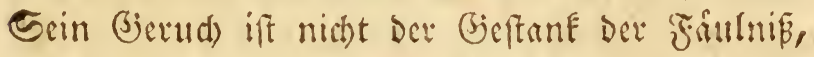
fondern ein geiftiger Saut des Sebens, uno nenn Das Sivcelium wic immer Din befannten fatalen meptitifaen Edbimmelgerud) verbecitet; fo uber= wiegt in Dem Encarpium Der (Siruth) DeE $28 \mathrm{C}=$ benmefles, mit armmoniaf mefre oder meniger ge= mifdst; mit einem şorte, fein ganjer Egarafter gleicht rem Der Earmenfeuthtigkeit, die bey Den PFangen in Den SElesschen Des Pollen uno bey Den Shieren in Den Seftifeln enthalten ift. Da

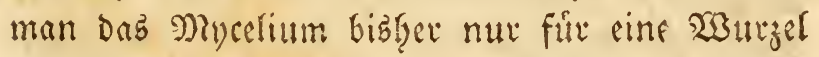

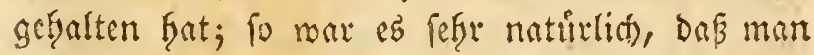

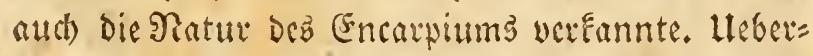
Gaupt zu fagen, betrad)tete man Dicics als Den

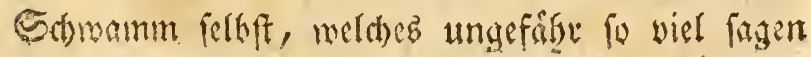




\section{XXVIII}

will, alb wenn man z. 25. Gey Der gemeinen Bechex flectle (Baeomyces, Scyphophoron, pyxidatus Ach.) Den Thallus fur Die 234 izel, Die Podetia fur Strunfe, Die Scyphos fur die Sorgane der Begetation, uno nut bie Cephalodia fúr bie Şlumen oder Fruofte exthicen nollte. Die. Gier zum Augenmerf genommene Analogie bes 5 (t)wam=

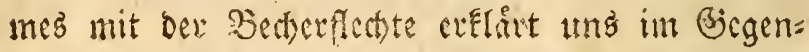
theile fefre anfourtion, onfs bas Mycelium in Nergleidung mit Dem Thallus, dic eigentliche

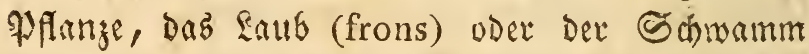
felbft, fingegen der aub Demfelón Gervorgedun=

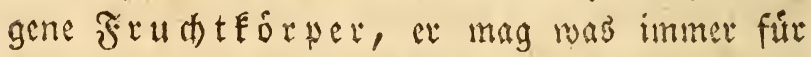
eine Biloung Gaben, nithto mefre uno nichts weni= ger fey, alb die fichinfrubt vermandeln:

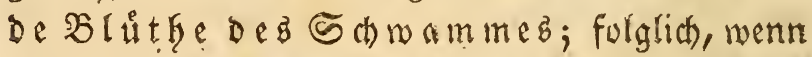
ein Ctrune vorfanden ift, io fey diéz ein Blu= thenftiel (pedunculus) mie Die podetia Des $\mathfrak{B}_{\mathrm{C}}=$

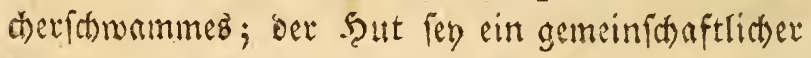
Blumen= oder frutbtboden (Scyphus bey Scyphophoron) uno die Lamelfer, Hoffen, Richer, Cracheln; Tubeveln u. D. gl. Reven an Der Etelle der Ropfonen (Cephalodia) bie Alusthei= rung ber sout in ifre finther. Die melentli= (b)en Theile Des Fructifirwerg find: a) Daz Sandammfleifh (Perisarcium), in weld)em die fibern aber nidst mefre in geftrict wie bey were 


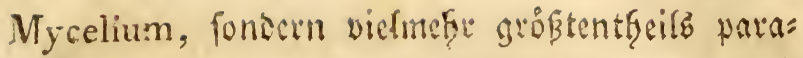
lefl laufen, und fich ebendefroegen diditer und fefter an cinander anfalicisen. b) Die befruatende Sarmenfendatigfeit. Diefe wird crft da aug̈ge=

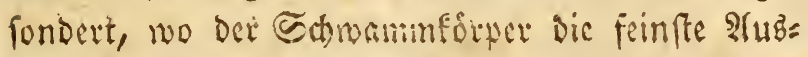
bildung exlangt bat, und iff eigentlicl) Dex reinfte Errtact won Der geiftig= garertartigen Subftans Debfelben. 2)?an Fann Demnach in Den meiften Fillen Den Frudtforper aud) mobl cin Etaub= gefißs (Stamen) nennen, wowon Der Ctrunf Dab filamentum und Der Şut Die Anthera ift. Det Woflen befindet fich innerlid) an Den Eriten Der Ramellen oder De: Röbrichen, uno entfialt die aura seminalis, Deffen Rifgel(ben jedoch) nicht erfit auf Den Rarben Dex Fruhtfnoten, fondern meis ftens faton an ifier Bjeburtsftefle zerplasen, und Durch) Den (jeift, Den fie ausfrauchen, die ganze meibliche Anlage bes (Encarpiumb befurtiten. c) Die Eøuำ (Hymenium); unftreitig ber aubgebildet= fte won Den feften SGeilen Des Frudterorpers. Gie ift, fo viel Dayon biafier befannt ift, bey als len Gabaammen androgyn, und alfo im ftreng= ften Cinne der Thallus De: Edtroinme. Der reele Unterfwied Der won iff ferworgetriebenen mannlict)en und weiblidisen Drgane fabeint allen Beobachtungen zufolge Darin zu beftefen, Dafs Die mannlict)en Slägden vorzuglid die geiftigen 


\section{$\mathrm{XXX}$}

und riefienden Etoffe, die meibfithen lingegen, nur cine concentritte fpeciffese Ballerte enthalten,

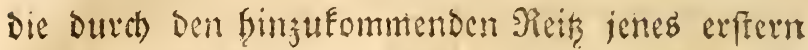
zum neuen Seben errect nird. Denn wir mif

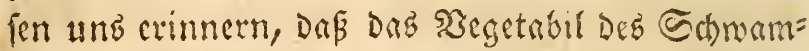
mes cine Ballerte fey, die mit Fibcrn gemengt

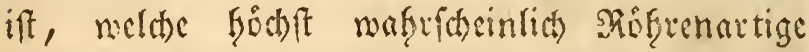

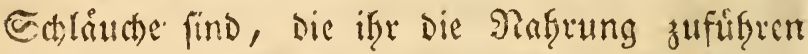
und bereiten. d) Endlich Dic Srut (sporulae) fribit, bie, wic gefagt, mit bem poffen untermengt aus bem Hyrrentum Gervorfcimt und fur $\mathfrak{A}(\mathrm{n}=$ barung neuer Snoividuen beffimmt ift. (5) ift,

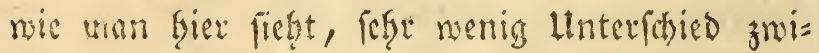
foran Den mafren Gamen Der Phoenogamen und Der Situt voer den Ectinammfrimen (Sporulae) Der Ectburmme. Fiur Sinofpen fann id aber dic Єdymammtcime nidjt mobl fyalten, weit

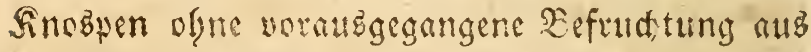
Der Cubftanz Des vegetativen \$yeils als mirfli= the Infinge neuce Jndividuen Gerworkommen, Fier aber ein Hymenium gragegen ift, Das immer

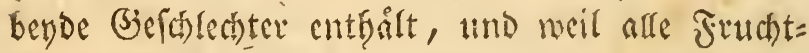

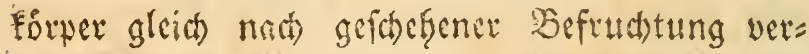
wejen doev verwandelt nerden, Da boch) jut (Fr: naffrung und Yabbildung Der Finofwen cine viel lingere Dauer Der Communication mit Dem $\mathfrak{b}_{0}=$ Sen, aus dem fie fervorfommen, erforderlict, ; is 


\section{XXXI}

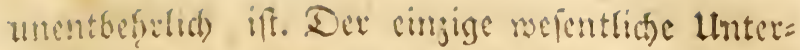
forco in Den Gamen Der Edtwaimme yon Den

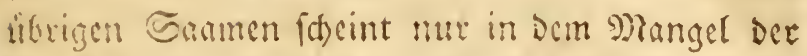
Butillesenen ju beftefen, nelebe aber nitht nothig fini, da biex bas Corculum cin purer fluffiger Sionter ift.

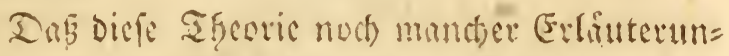

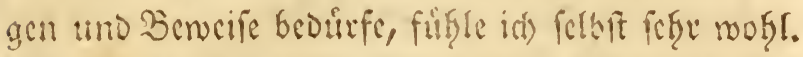
Fich) merbe auch noch (jelegenfseit finden, einiges

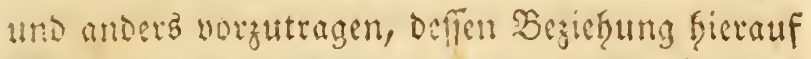
firi: werentliob ift; nllein mici) in cin weitlüuftiges Intail cinzulaffen, daz crlauben mix die Biranzen

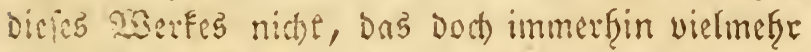

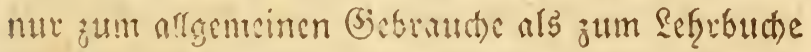

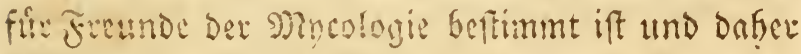

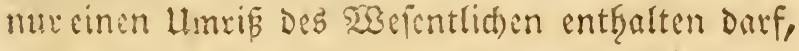

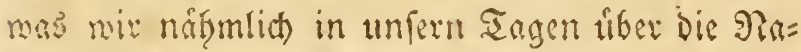
tul Dex Gobramme fagen Eommen.

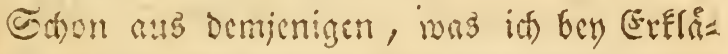

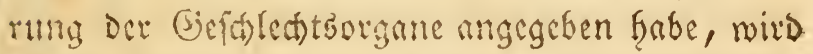
manmane SGeoric über Dic Seugung uno jortpflan=

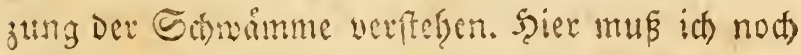

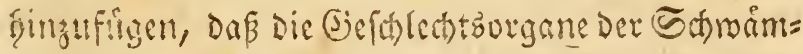

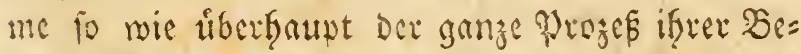

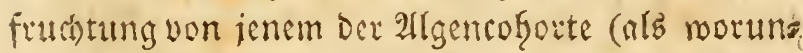
ter ich in meincm Methodus Dic Phyceas, Licheno= sas, Mycenas, Sarcocarpas und Gasteromyces 


\section{XXXII}

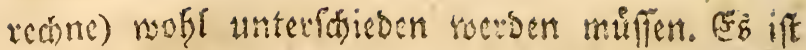

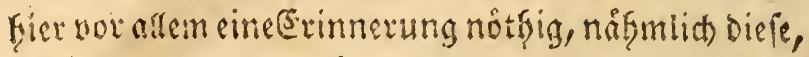

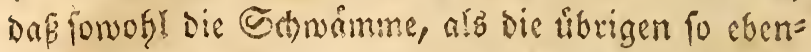

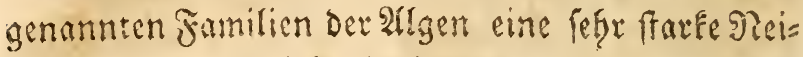
gung faben Broillingfgeburten Gervorzubringen. Es firt dafeer ferie oft meffere Sporulae gufammen von cinem Batutbef̧âlter (Gongylangium Bernh.)

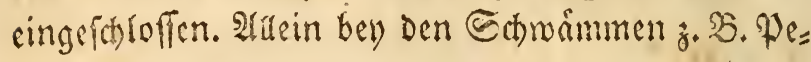
zizen, fint Diefe Gongylangia nichtz weiter als ver= madfene, dem Hymenium einverleibte Sporulae. bey Den allgen bingegen vertgailt fith bie Sarthe ganz anders. Şier find die Gongylangia abgefon= Derte Srganifationen, Dié aus dem vegetativen Iflei= le Der $\$$ ffanze ferwortreiten, mandimafil nod) mei= ter zulammengefefit fint und ein Receptaculum secundarium oder einen polfter (Stroma) bilden. Alud befinden fich bey ben algen bie benden Se, rualorgane nitht fo burch einandex aubgegoffen wie bey Den Edtwaimmen, fondern gróptentrietls in ges wiffer idon etnas ausgezeichneter (Entfernung von cinander.

Gleichen, Bitfrato u. m. a. befgauten, baF eine gerinfe Portion der fifermatifaen Fluffigereit von Den Stigmaten cingelogen und zu Den Embryonen Der Saamen in bem Frudtefnoten fingefradt wers Den milfe, menn diefer fuudtbar werden folle. Adein Diefe ŞGeoric ift offenbar mieder zu materierl uno 


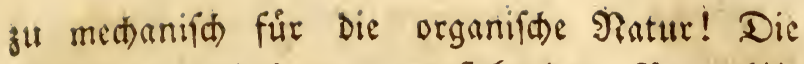
(jorundinge jedes neu entfethenden Negetabils oder Thieres ift cin ausfhliefendes Eigentţum Des weiblidten Rórpers, Dem es einjig an Der Miaterie fefflet, melde erfordert wird, um in Denienigen Drganen, melde die Entwicklung Des Embrno bewirken můffen, eine folthe Tgåtigfeit Durch ifgen Reis ju crwecten ald fiegul erforder= lict) iff, cine Ighâtigfest, weldhe mit jener Der Selbfterfaltung im $\mathfrak{B i d e r f p r u c h}$ ftefert, und die Dann meictitig genug ift, um jene entweder ganz

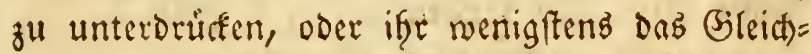

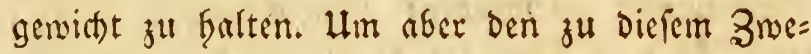

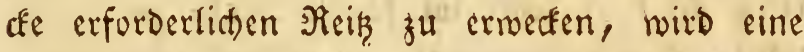
viel feinere Speration erforbert, als man fith gewo̊g̨nlic) vorzufteden pfleget. Eine Flüfligkeit Fann nicht to meit Durd Diefe úberaus feinen

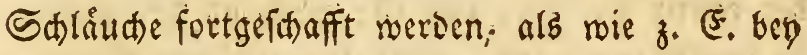
Den Biefáffen eines Girifela bis zu Den 'Fmbrnos nen im Frudtetfnoten, werigitens nicht, of ne Durd) Beymifatung anderer geinglid verándert

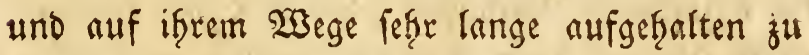
merden. WBir fetgen in Begenttheile fo woffl ben Den Pflanzen a!s bey Den Tffieren, daß ven Dem Atugenblicke an, als Die sefrudtung gefatefen ift, in Den Drgnnen Der Geburt fo großße כerinde: tungen vorgefen, bá wir geftefen múffen, Die 


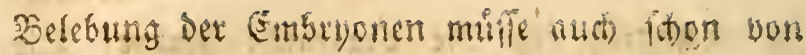

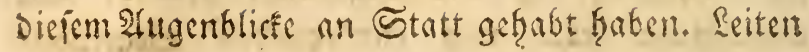
wir Dagegen biefe großen 2 sirktungen ven bem geiftigen feauche hex, Dev nub der fpermatifón Feuchtigfert fith entwidelt, uno bey Den Thies ren die Aerven, bey Den Pfanzen die feinften und reibarefen Gibilde berufteret, und fie Durcts Dice beruffung augenblictlit) zu ciner verefefre

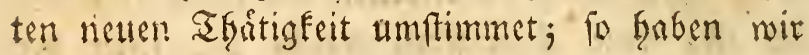
einen Begriff, Der uns Benüge leiften und un= forn weitern Unterfuchungen fut Drientirung Dienen Fann. Go fornnen wir z. (5. gleids) an Den Sobwaimmen veriabiedene Einribtungen bame: Fen, die Diefe Sefrec im Fohen Brade gu befta:= tigen, allen andern aber fu miderfarectien fohe

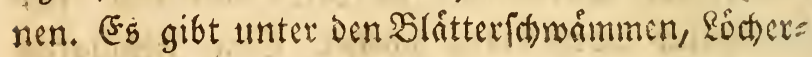
und Etachelfownammen mefreere, Deren frut in Der erften Periode, das ift: naifecend Der Perid= De Der Befrudtung, ring um mit Dem Strun: fe Dureh eine fraut bergeftalt verbunden ift, baf fonofl Der Zutritt Der inkern Suft, als auds Die alfulanelle Derduftung Des von Dem Hymenium ausbuftenden $\mathfrak{A} e t$ hers badurth verfindert

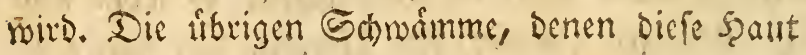
mangelt, Die man bald æing (Annulus), balo Sorfyang (Cortina) nennet, pflegen bald iffere

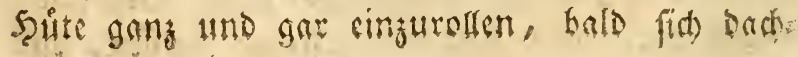




\section{$\mathrm{XXXV}$}

jiegelformig und feft anliegend fut bebecken, ober ourit) einen befonderen Filz, wie Feufde Mlid: den iffen bufen zu verfalevern. 230 zu diefe Sorficist, menn es nicht Darum zu thun feyn follte, ben belebenden Duft fo lange aufzubef̧al= ten, biz alle brutfeime won demfelben belebt und zut Entwieflung neuer Sndividuen bifponits

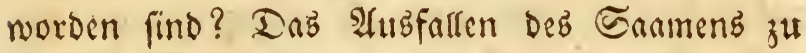
verfirten, Eann nicht Die Itbficht feyn, Da diefer Damafiz nod) nicht reif und auf ber Dberfacde Des Hymenium Fnum zull bemerfen ift! . Sicle Sotwormme, 3. 2. alle Ifrtenvon Clathrus, Phallus, Amanita fino wáfient Diefes Zcitraumes yon einer 23 ullftiaut (Volva) gónglid) eingefकlof fen, und bey diefen ift aud wirflid) dic aura semi-

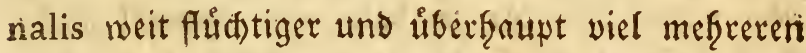

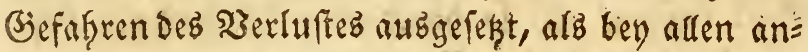
bern Battungen Der Schmaimme. Die Pezizen id lie= fen iffe Mlundungen, die Srelvellen und Mlordedlen falten fich fefre enge zulammen, andere, wie bie Ela = varien, Spatfularien, Reotien u. f. m., verbergen fich unter fremben Fiórwern, z. 20. unter den Mloo=

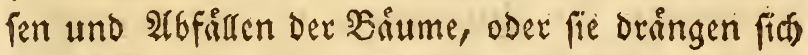
to feft zufammen und wachen fo gefertig, bas fie

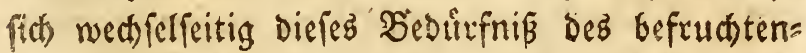

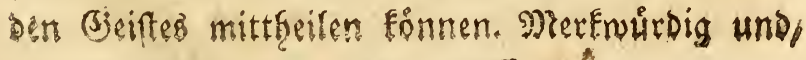




\section{XXXVI}

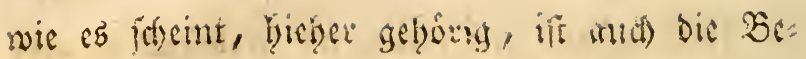
vbachtuig, Dap bey Denjenigen Ed)wammen, die in groben Saufen bebiammen oder aub cimerlen Diaffa vom Mincelium berwormadten, afle mit= farmen, fo wohl grope als flcine, in eirrem glei= ben Beitraume bie Function Dex Befruct)tung verridhten. Eoute man nicht ficrab den Eibluß jiefen Dưfon, baß̧ iencr fo wunberthatige afe= ther, indem er autb nur in einigen Steilen ci= nez fulammengefesten Frudtebrpers Belebung ernirfe, Demnod) anf Den ganzen cinen fo ftar= fan Einfü Gabe, Dap alle weitere Entwicklung auf cimmafl auffiovet, und nur aflein dic $S_{c} c=$ gưffigung Der befrudisteten Brut won bex gan= gen शialfa bes Encappiumb betrieben werde? Dit cincm $230 r t e:$ fo balo alb eimmabl diefer

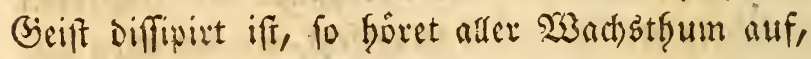

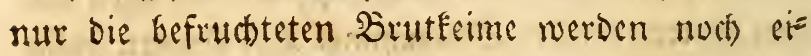
lig fum Biel gebracht, und Der ganze Fruci)tfor: =

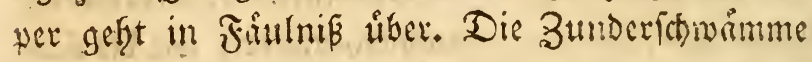
fonnen meincr ß̧ęauptung nicht entgegen gc= ftellet werden! Denn ifie Wiebcrauflebung und Erneterung Der Functionen gefdieft niemafle aนร Den nafimliden Srganen. Der alte Frudt= Forper verbartet nur, anftatt zu verfaulen, uno wiro jum Etandorte, ofne mef̧r cin Echromm

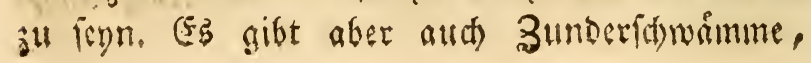




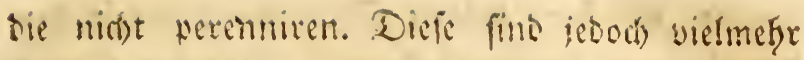

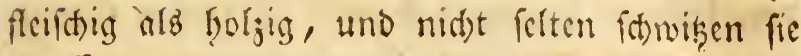
23affertropfen aub, wo man fie Denn audh, jes Dod) nut uncigentlid), TGrånenfdwaimme nennet.

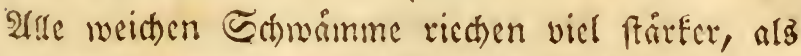
Die Sholgartigen. Bey gennuer Unterfuchung wiro eb fid aud zeigen, baf bie lekgtern weit meni= get won Denienigen b̉lésd)en bervorbringen, die Dem Polfen Dex Antheren entwechen. Bey Den umgetefiten Sdurummen, j. 35. bey Poria, fann! ferner cin fur alle Mathl Fein flüfigez, fondern

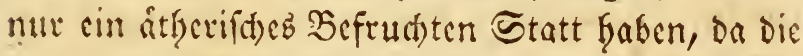
Eage Diefer Gabramme der Sage Der ubrigen ent

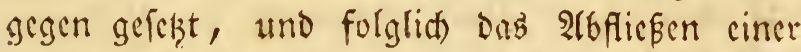
foldhen Feudtigkeit nidht geocnfbar ift. Hlebrigens liefe es fich ja fogat mathematifd) Demonftrien, da cine Fluffigkeit, die fo fein ware, Daßs fie wor ocn গarben Der Sibrammbrut eingejogen me: ben Fonnte, fdon menigfteng bis zut Eubtilitât cines Dufres ycrdunnct feyn múpte - und ir Diefem Falle verdienet fie alfo auta nicht mifge sin Liquor genannt gul merden. Sif es aber ein Duft; fo finto wit fabon an unicrem Biele, uno eb formmt nun mus auf Den Dit ait, welder yon Demietben berufget uno gersig̨t werden muffe? Der fieim felbft, oder nur sas if̧n entfaltende Drgan? Da aber jener fowohl feinen exfen. 4he 


\section{XXXVIII}

fprung, als feine fortete Entwicflung eingig von Dem lesterteren ferleitet; fo fefge id feinen (biund ein, warum wir Dem Sieime diefeg Stimulans

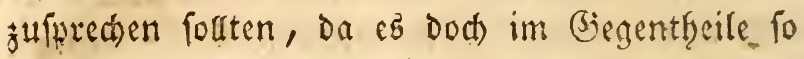
nothig ift, Daß daz mitterlid)e Befraitnis cine erfiofte febfaftigfcit crfyalte, um cinen neutn Siokper zu bilden, und aus feiner eigenen Guto= ftanz zu crnatgern, und ba eb ju dem ficime auf Eeinem andern $\mathfrak{X B e g e , ~ a l s ~ D u r c t ) ~ D i e f e s ~ w e i b : ~}$ lictye Drgan gelanigen fann! Die Niefultate, Die aus Diefer Theoric entipringen, find wen Der. grópten Saichtigkeit; aber es ift fier nid)t Der Drt Dazu, fie alle augzufúferen und ju beneifor,. und id muß es Dafer einftweilen der Hettgeilg= Fraft meiner Sefer felbft trbe:laffen, fie Gerauz zu zieffen und zu verfammeln. Э?ur ein Waar Davon. fer) mir exloubt, asforiftif zu ffizziren, weil fie mit der Sienntnis der Canmeimme überf̧aupt To feft verfniwft find, Dak mir Dadurch in Den Cramb gefest merden, mittelft Derjelben unfer Uttheil Darưber mit Demienigen, was unz von ben Wfornogamen befannt ift, in ein ebenmâi ges. Nerfiailtrib gu bringen. Die Edwaimme ge= bionen Demract)

- a) nxt Der Strenge bes Sinnåaniften Seruals Eyftemes in tic Gynandria Monandria. Dod mup man nicht vergeffen, Dap id fier nur von 


\section{XXXIX}

ben sigentlithen Cobraimmen wese, uno alfe bie ictson bben genannten algenfamilien, beren einige man bigfact ben Eanwammen beyzuzåglen ge= nognt wat, savon ausjibließje.

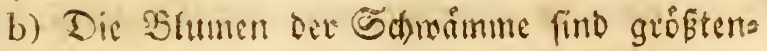
theils nackte Blumen, D. h.: fie Gaben feimen Ficld) und Feinc Sfumenfione. Dod machen Clathrus, Phallus, Amanita u. 5. gl, bievon cine sis nigftens eben to gut fur eimen Calyx zu falten, uls Die Edthligen Der Aphyteja Hydnora. Der Echniamm felblt lit ein. Staubgefás, das mit Dem pouen wermengt, die J゙ruthtfnoten ausfeckt.

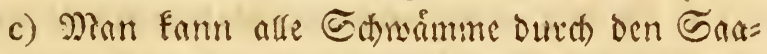

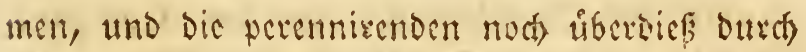

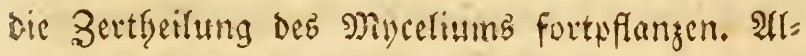
lein ein Fruchtforper, Encarpium Fann nid, ver: fest, genfropft oder geftecft werden; benn ex ift niăts weiter alb ein Etaubgefóf, ober eine

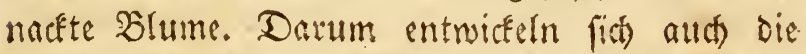
abgefdnittenen Edowamms nicht meiter, waikrend

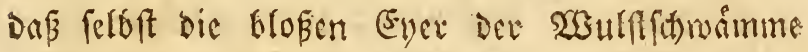
(ich) aud now aufer ifrem Ctandorte entfalten.

d) 23 enn wir bie fier gegebene Definition rines Sannammes Gintanfegten; fo mäkten wir mand) Pllanzengattung Dem Siebiethe Der Schwaim:

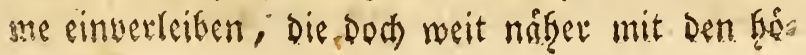




\section{XL}

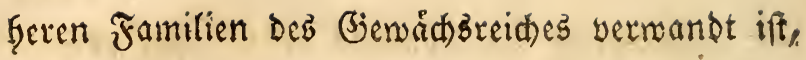
j. b. Cynomorium ift nach feiner ganjen $\widetilde{S u b}=$ ftanz cin wafrer Solnamm, und hat nod uber= Dieß mannliche und weiblid)e (siefólectstzorgane fo Durct cinamber gemengt, wie eine Clavaria. (5t) wab weiter entfernt waren bann aud die (bat= tungen Arum, Caladium, Pothos, Calla, Ambrosinia, Zostera, Dorstenia, Ficus, Mithridatea, Cecropia, Zamia, Typha, Aponogeton, Artocarpus, Cytinus, Monotropa, Bromelia, Araucaria, Banksia u. D. gl. AUtein bei mafiec פaturforfder begnügt fid nidet mit fold)en cin=

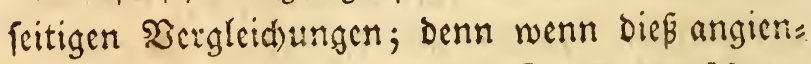
ge, fo míste man auch) bie Battungen Marcgraavia und Eucalyptus, um Der Calyptra millen, ben Laubmovier, Die Fópfigen $\mathfrak{A}$ ten von Siatice wegen Des ßinges Den Agaricis und to viele Arten von Ruscus, Xylophylla, Phyllanthus, Platilobium u. b. gl. Den Farren ein= verleiben.

Mrit biefen wenigen ๔åken zufrieben, werfe id) mir nunmefir eine neue, nidst meniger inte= reffante Frage auf: $\mathfrak{3}$ ie pflansen fid bie Sd)wånmte fort? Die Intmort bierauf ift mit groben Sómierigkeiten verbunden, Dennod) Goffe id, Einiges und Anderes Davon erflaren $\jmath^{u}$ Eonnen. 


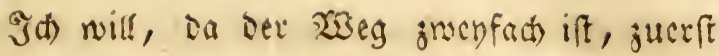
dic Fortpflanjung Durch Das Mycelium erotern, und Dann aud) uber Den andern, die Derbreitung

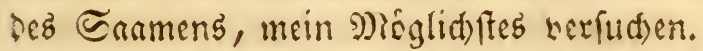

Edroimme, Deren Mycelium nur annued iff, mie bey Den meiften 2 trten von Ag. Mycena, Omphalia, Coprinus, von Phallus, von Peziza, yon Clavaria u. ๖. gl. Fonnen fith offenbar eben fo menig als andeve fogenannte Sommergemaidie, fo menig als eine Euphorbia Helioscopia Dutd)

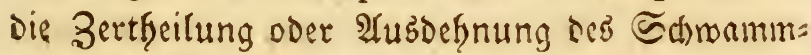
gewåchfes vermefren! Dicjenigan fingegen, dic nie cin Agaricus campestris, ober Boletus Tuberaster cin perennitendeg Mycelium Gaben, fon= nen auth Durch bie Bertfeilung Desfelben, wie eine perennivende Plange Durd die Bertheilung

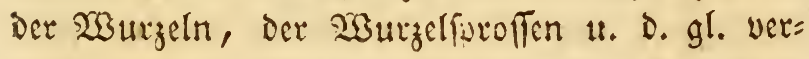
mefiet merden. Diefes gefareft Denn aud wirflid iefgr barufig, theils abfictstich, theils zufällig, leks= teres Durch Heberfeß̧ung Der Barume, Durch Ner= füfirung Der (Froe, Durd Den Nerbraud Dez MToo

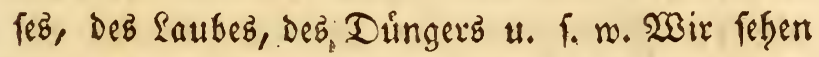

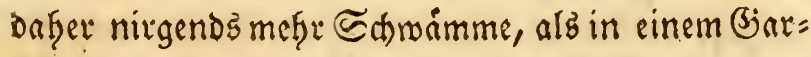
ten, in melden die Biewaid fe aus feffe verfobiede= nen Biegenden mit fammt bem Sallen verfekst move Den (Werfteft fich, menn Feine andern Sinderniffe,

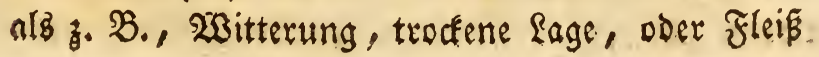




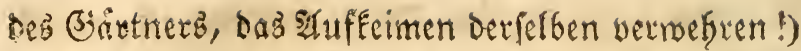
uns ber f. F. Puftgarten von Gobonbrum ift cin reonder Seweib fiewon! Die parafytifden Schmåm me Det SBaume fónnen nut auf Dem individueffer Baume fortwudern, Dem fie angebofren wurden, und, Der Boletus fumosus Fommt in oem movern= Den SBeidenbaume alle Safre an einer andern Stelle mit feimen Encarien zum গ̧orfacin, bis eimmagl dex Cramm abgefguen oder fein Myce. lium Duxh einen fefre ftrengen 2 Binterfiof $\mathfrak{g e}=$

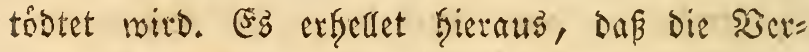

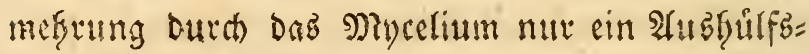
und Piebenmittel iff, modurch Die Ratur die Sdownmme vermefret, aber lange kein foldbes, nuf weldes fie bie fichere Erfialtung ifier noth=

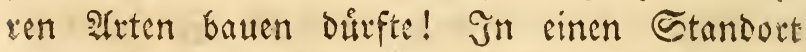
ribertragen, ber feinet Ernábrung zuwroer ift,

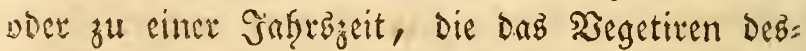
fitcen verfinbert, fann ez fefgr lange rufen,

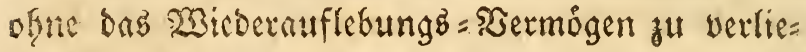
ren, und nut ftarte Shikge, oder ftarfer Froft,

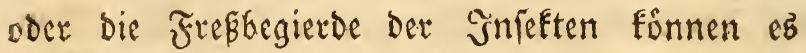
gånglich verridten. Fâulnis und 2 Baffer verder: ben es, aber (chwerlict) ganj und gar, uno bie

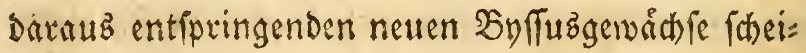
nen nur corrumpirte und monftrofe Eonforma= tionen zu foun, die nid)t mefir Rraft genug ba 


\section{XLIII}

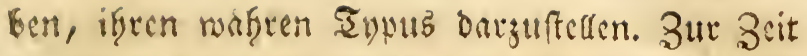
'Der belebung vegetirt es mef̧e oder weniger rafh, je nadbrem bie Fraft ber feutsten siarme ffir: fer oder minder ift. Seboch faseinen die meifen Mincelien riberfantut nur feffe tangian, uno in oft unterbrod)ener Igatigfeit fortjuwad) fen und fiit) allazubreiten. Sie Dringen in Die Froe, uno vermengen fith mit ben Partikeln berfelben. Dod

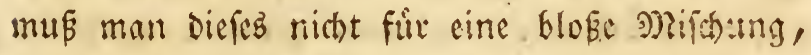
fonbern als eine Erfosinung anfigen, dic mit derienigen ganz cinerley ift, mann wiv Etüct=

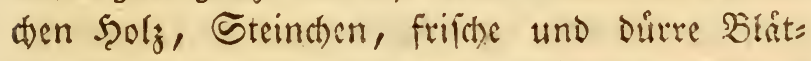
tev, Brabfalme, Sogelfobern, Frưthte und Saa= men, Noofe, Gnfeften $=$ Cabaver und Gundert

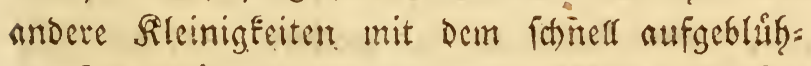

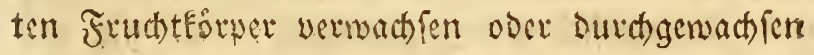
erblicken. Sft ein Mycelium Parafyt einez leben: Den Baumes; fo lét es entweder in Dem Mros Der Debfelben, wie jene in ber Eroe, ober in ges fieften uno abgeftorbenen Shógern, ober cb ift fo gang und gar Paraint, wie die Miftel, und nafget fich von ben unverdorbenen Saften des

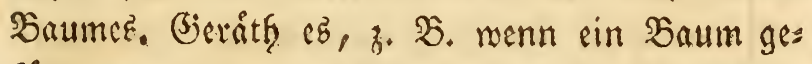
fillt wovden, Durd Hefertragung in eine foldte lagge, in welcher es nictit alfe bic Scbingniffe feiner Entwicklung im vorten Maße antrifft; io

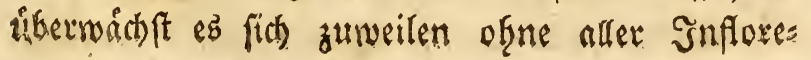




\section{XLIV}

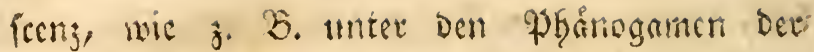
Sopfengl Dor Epargel, Das sitterlúp uno wie Bemaid) je, Die von ciner finftern Etelle cinc lan= ge Strecfe fin fortfriechen, um fich Der Epalte zu neffern, nodurch die Ruft und Des SEnges: lid)t einbridst; oder fie bringen zmat eirten.Frud)ts forper, aber nidft iferen eigenen, fondern ein Monstrum, eine Peloria! W3ir fefyen Dafer manct): mafl mit Ereftaunen auf Dem gefoilten Şolge in Der Selgfammer ganz andere Schwammgattun=

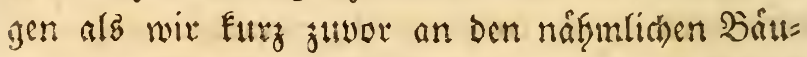
men, da fie nods in Walde ftanden, bemerften. Pe!orien Der Pfionogamen entfetgen, wie id) felbft beokad)tet babe, menn fremoartige Pfan= jen fo Didst Durcheinander mad) fen, Daß ber Filz

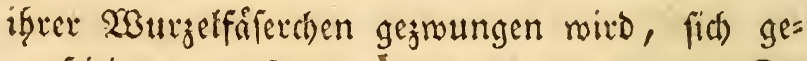
genfcitig zu umfangen "und zu Dutchmeben. Da Die Mrycelien Banfertartig und faft flüfig find;

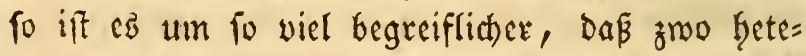
rogene Mineclien fich - nid)t mifhen - aber Dod), wie wir oben yon ber Erde gefagt fanben, surctinachien fonnen, und in bicferm Falle (wets

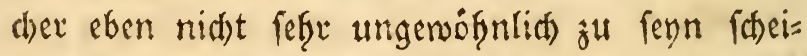
net!) mógen mogl aud Die Salwåmme, io gut wie Die \$gonogamen zu Pelorien werben. J(t) faf nidft felten zmey und Dreyerley Arten son Sd)wimmen aus cinem Stridfe bervorkluffen, 
sber foft inuner nur won ber gleitinntigmigen (3attung, 3. 5. 2 Ágaricos oder 2 Boletos. Dee âltere, Der hier fdoun linger Domicilirte, fetien Die Dferfiand zu gewinnen und Den jungen 2 an:

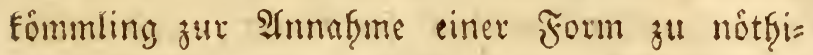
gen, Dic ber feinigen affnlith war. Dft mögen

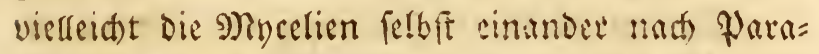
fotenart ausfaugen, und bey Den Gathámmen fann man biefer Эaţrung allerbings cinen viel mirtigeren Einfus auf Die Eunformation Des Encerpiums cingeftefen, als bey Den Pfionero= gamen. Denn wiv fefen fithon bey Den Barum= f(d) wâmmen, mie viel von ber \$erfdeiedenfeit Der

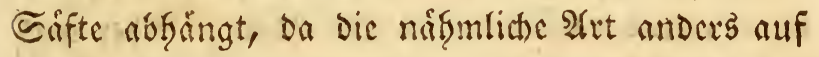
Der Buthe, unders auf der Eithe, anderg auf Dem $23 e i d e n b a u m e$ und mieber anders auf bem Mingholderbaume fith entmicfelt, wie wir diefes Fefir leidt an Daedalea quercina, Boletus fomentarius, Boletus citrinus, Boletus hepaticus, Sistotrema versicolor, Agaricus ostreatus and fundert andern beobadten fornnen. Waun= Dern wir uns alfo gar nicht, uno futhen wir die $\mathfrak{H}$ rafacten ja nicht in großer Ferne auf, menn mir auf cinmafit im $\mathfrak{W a l}_{\text {ald }}$ neue Sd)måmme be= obachten; Denn wenn ber Saame won einem Bo-

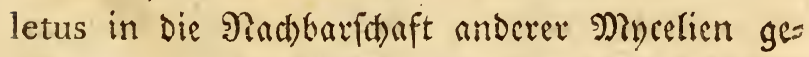
follen, fo mubte das Daraus entftandene jüngers 


\section{XLVI}

Mycelium feiden, und allenfalle ju ciner That lephora werden. Afber biefer SBaftard miro bals micber veridwinden (aufer, wern er aufa nelse Durct) gleide Zufáfle erzeugt wiro!) uno die $\mathfrak{U} r=$ arten merden daneben fortbauern, uno immer Die nåfulithen bleiben.

Die periodifat Erifheinung ber Sthmamm= Forper beweifer uns indeffen ganz beutlid, Daf wir nidt algzuviel auf ben Einfluß ber 23 itte= rung anrecinen bixfen! Die Sinwaimme fommen auch bey einer nictit gan gunftigen 2 Bitterung in ber beftummten Jaf̧rézeit zum Norfacin, nut blciben fie Eleinet, nur exifheinen fie etwaz fwat:

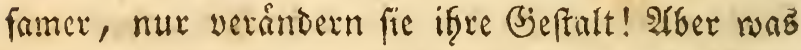

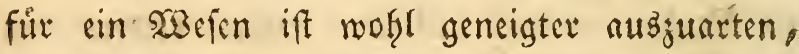
als die Canmamme? Ja) folluffe Gieraus, Daf

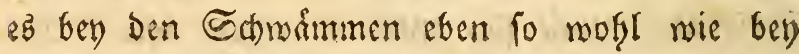
Den $\mathfrak{u} 6$ rigen Pfanzen mafre fpecifife unteriatiede getbe, bie in ben innerlidien Eigenimaften iffer siegetation gegrindet find, und das Mycelium,

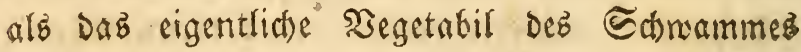
binfinglict) verfd)icoen feyn mûffe, um ung fü Die BuFunft grundlide d)arafteriftifithe MerEmafile jut Unterfdeidung Der $\mathfrak{A}$ rten anzubiețen, Da wir von ber Form ber Frudttiprper, wie aus Dem Norigen etffellet, nur affuleidt) betrogen

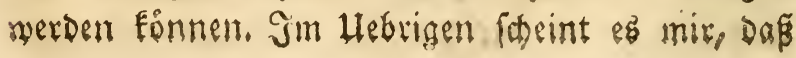




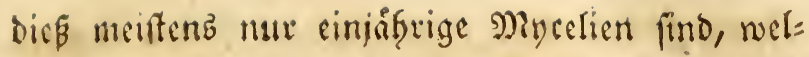
d) logar-genau die Weriode Des Emporblúfens beobactsten und die ferennirenden baften ifge $\mathfrak{P}_{\mathrm{e}}$ rioben lange nidt fo punftlid); aud) (d)eint es bey Dicfen wcit inefgr auf Dic witterung anzu= Fommen, und wenn fie alfo audb einiger Maffen

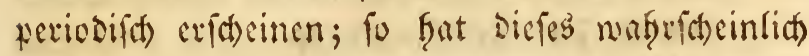
nur in Den siethåltniffen Der ben Jafrejecten cigentbumliden 3 Bitterung ifge (jirunourfad) und nicht in einer angebofinen periodifaen 2 bwed) lung won Ifgátigfeit und sube. In wie ferr Die Mrycelien frember Simmelaftrithe fid an die Fatur eineb andern geroobnen und accimatifi = wen laffen? Dieß fann negen Mangel binlägli: der Beobadtungen fut 3eit nicht entfáieoen werden. Die Parafitifasen, fheinet mir, würden meit fdwerer Dafin oju bringen feyn, als bie Erofanomme; weil fie won jef̧er Etandort uno Nafirung mit Der Mutterpflanze getfeilt Gaben, und alfo ein शaturel fraben muffen, das Dem

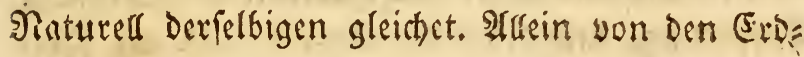
und Moderfdonammen, dis eiren Differn und feuctiten Standort Gaben, und Deren Mrycelium vielfeid)t niemaf̧] Die Mad)t Der Gonnenftraflen exfährt, won biefen, meine id, modsten mofbl

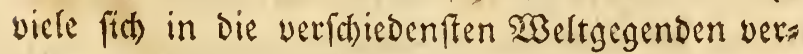
pflangen laffen. Beftatigung fux dief̧e నermu: 


\section{XLVIII}

thung finien wiv, menti nic bie Flora Danica mit Den floren vom füdictsen ("uropa und felbft won Cibirien, Dem Drient und Effina verglei= wen. Sefir nuturtid)! Die Sawamme leben ia eingejenft in ifrem Etandorte, wie die Tange im Waffer, und man weís ja, daß geniffe Tang= arten Durat Die Edrifffafiet in ganz entfernte Meere úbertragen uno alloort zu einfeimiften gemacít morden!

Die andere und bey meitem die genoifnliá: fte $\mathfrak{A}$ et Der Fortpflanzung Der Cothroume ift Die Durch den Sammen, doer bie fogenannte Sthrommbrut (Sporulae). Dic Samen ber Schnåmme zeidjnen fich von benen der úbrigen (jewaidble Durd) Die Eriftaunungzwúrbige Zarts Geit und Durch Daz Corculum aub, weldes eine pure verbidstete Gelatina ift, und fid felbft die Stelle der Sutyledonen vertritt. 23 as den erften Punct betrifft; fo fheinen ifgnen zwar Dic Algen (in ber won mir oben angegebenen aubुgedef̧nten Bebeutung) und felóft die Moofe und die Far= ren nid)ts nadsugecuen; alfein bicfe entf̧alten Dod) immer iffere viele beyfammen in einem $b_{e}=$ fialter (und in biefem Falle find aud) bie San= men Der Pfönogamen immer viel Eleiner alb an= Dere!), Die Camámme bingegen tragen faft lau= 


\section{XLIX}

ter cinjelne Eammen, uns bennod) find Diefe ven Der Alt Der allerEleinften!

Dar Siemen, Der Eamen glaube id), menn es ja exflatt werden folle, auf folgende $\mathfrak{A} r t e r=$ fliren ju fonnch: Seber Sawammianme ift ein nacter Reim, Der aber Dura Den Butritt Der Suft in feiner Sberflache vertrocenet, und foldac: geftalt ein uneigentlitises Eammentorn bildet. (jes= raith ein foldes fiown an Den Drt, wo J゙eud =

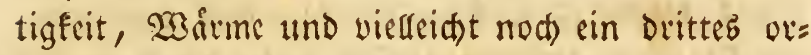
ganifa)es Anflofungsmittel diefe Rinde Durabeis d)en, fo exgiest fich Dic (salferte und tritt als Reim in Das Meer Der (Semente, dic fie füttern. Atrein bey einem Raijonnement úber die 2 Birfungen oder Eriderinumgen Det organifitten 2 befen follte es นnz genúgen, fagen zu fơnnen; roarum etrons

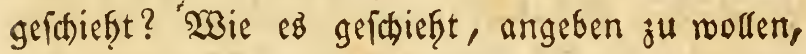
beifst abermafi die Drganifation zum tobten Ples ()anismus fieraburbigen. Das Gaaman= ober Srutforn bes Sabuammes fann fich unftreitig feffe lange, ia wohl viele sange exfbalten, bis endid einmahl alle die bedingniffe gufammentref: fen, weld) zu feinem Ifuffeimen exforderlich find. Fines biefer Sedingniffe fheint benn aud die Jafres zeit zu fenn, und diefe perivoifare Reigung zu Fei men ift eine Eigenidgaft, die nois nicht anders als 
aub Der angeerbten Lirtraft ber feciffen Miateric jober Ift Gerleiten fonnen!

Die Srutformer ber perennirenden Mincelien mogen vielleidtht auf Der Stelle Feimen, wic fic ausfalten, und nur exft bann ausblutgen, wenn

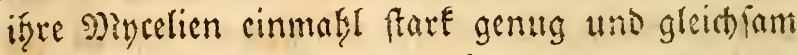
mannbar fino. Siellcicht gefforet Daju anth nod

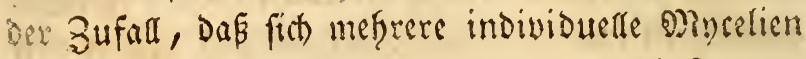

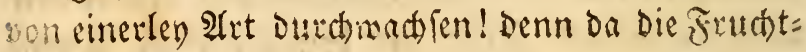
forrer mefereret Sotwammarten aus verfólede= nin Srganen jufammengefekt fino, die bod alle sie Soruterner abrerfen, wie z. B. bey Den Mor= chein ber F̧ut und Der G Strunf; fo fann

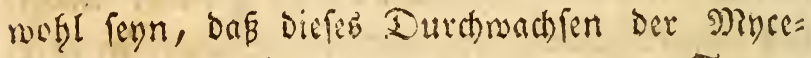
lien yon den Fiornern Des hetutes und des Gtruns feb erforberlich, ili, menn wieder eine Morche! nufwachen und bende Sogane entwickeln foll.

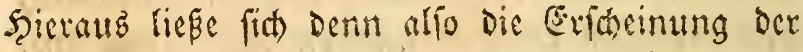
unvouffommenen Silbuningebilde exflären. Sá) Gabe mefrumaflen Boleten ofgne alee Sinlage von

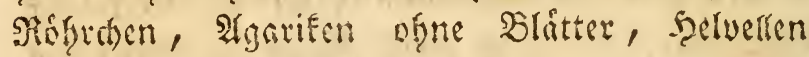
ofgue fruite gefefen, sic bod librigens fo gut aubzgefildet waren, Daß̧ man die 2 trt genau be Primmen Fonnte, jui Der fie gefforten!

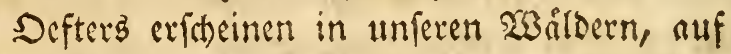

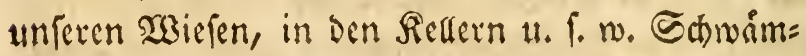
me, won denen man bisg̨er Feine Spur in Der 
ganjen Biegenb finden fonnte, und bie ber zufils ligen Befdaffentecit biefer Standorte fo angemeffen zu feyn icheinen, oar vielleidst in taulend gafien feiner ba werdesen fonnte, uno in wieder taujeno Safieen Feiner mefre da madyen durfte. Sind etwa Die Ect)wámme Segetabilien, ofne alle beftimm= te Form, und faingt diefe etwa ganz von bem (sinfluffe der åuferliden Umftinde ab? Der wafje Iffrinenfdwamm (Merulius destruens P.) waid)ft nur in Bebriuben uno fommt unter gemiffen $\mathcal{H}$ !n ftanden Dafelteft zum 2orichein, wenn in Der शiafge

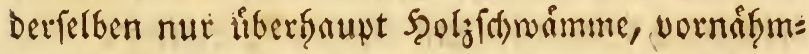

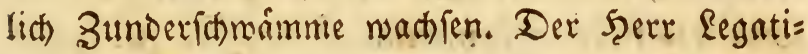

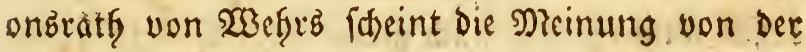

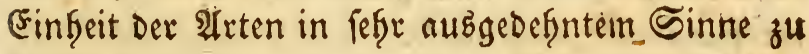
begunftigen, wenn $\mathfrak{e r}$ in leiner $\mathfrak{A} 6$ fandlung $\mathfrak{u} b \mathrm{er}$

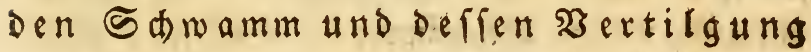

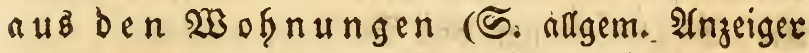
Der Deutithen, Jafirg. 1806 U. Nro. 28g bis 292) zmifthen ben Schwammen ber Wailder und Dem

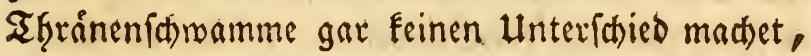
fondern Die Entftef̧ung dez leşteren von dem Saa= men berleitet, Der mit Den Balfen und Dielen

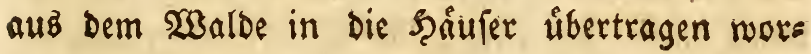
Den fenn foate, und wenn er in biefem 2tuffakge ůberfaupt nur vom Sanamme redet, er mag den Tfrainenidomamm doer nas immer fü einen 


\section{LiI}

andern bejeichnen. Shiafer frat aber noch niemants

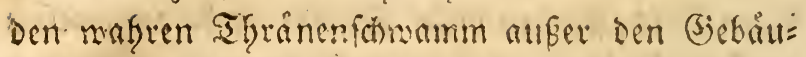
Den, Sergwerken, Fituinen u. D. gl. im $\mathfrak{B a r t s e}$ getroffer. Man findet gwar in fefre warken ino

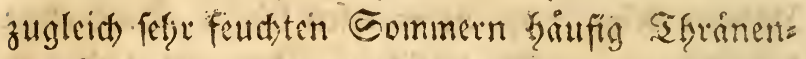
fdowme, affein biefe find nicht nom Merulins destruens, fondern angefogene und ficifdig ges moidene Yffarten som Boletus fomentarius $P$.

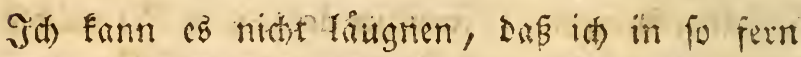
gan ber noffmlitfen meinung fey, menn man

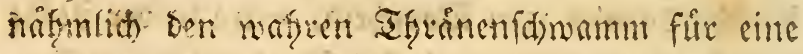

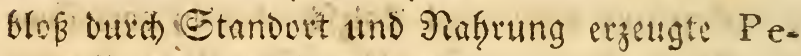
foria bez Roletus fomentarius aubgibt. In Zolge Deffen thus ich abet aud gleid einer Mienge ande=

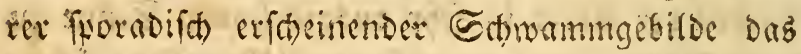
jus speciei abfuret)en und hiemit Die wliklit)e $(E x=$

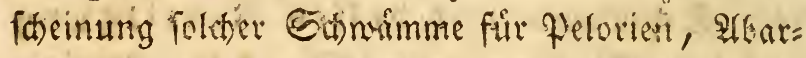
ten, Misdifationer und Baftarde erflaren.

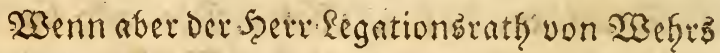

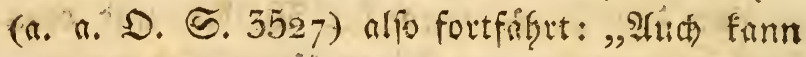
eE feun, und wer nito ez yerneinen? Sab dex Saa=

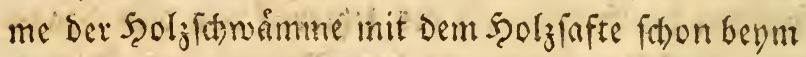
Itufwachen Des Solzes in dicfes fommt, und fo range darin ftill liegt, bis ifgn bie frolzfäulung

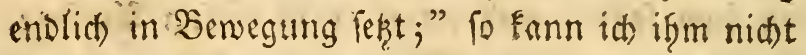
benfurfen, obgleidy es mir nidt entgangen ift, wie 


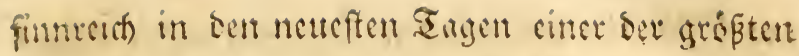
Sotunifex, Decandolle in Dem Vemoire sur les Champignons Parasites (f. Annạles du Mus. V. Annè, Cah. 49. p. 56 et seqq.) von Den Aecidiis, Xylomatibus, uno Den fibrigen ât gen diefer Frmmilie ungefabr bas nifmliche be= Gauptet. Fine Sntubfufcetion Des Sarmenz jus geben, fiebe nad meinem Hetfeile fen Rieis= Inuf ber Gafte in ben organifoen Siowern fur: einen furen Mectanismus cteliven! Mic fann ein frember Rorper in den Drganen eincs an: bern jugcaen feyn, ofgne ifn cntweoce ju jerlto: ren, ooer von ifm zerffort ju werden. Ia a mas Durch Die (Biefifie eines organifthen Rörpers. burchgefet, wird in bic Subfanz wermandelt, fomogenifit, uno mern es unfigig ift, fromo= genifict gll merden; fo entfeft cin 3ney Eampf ber Raturen, unio ber frembe Siorper, ift ein

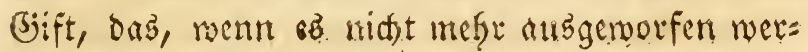
Den fann, Den entfaltenden fiurper widernatix:

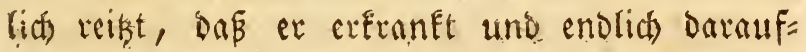
gef̧t. Man muß fid) oie Eaftgefaßpe Qex Pflan= zen, die dicfe Ganmen fortbewegen follen, niaht wie Sd)laudbe vorftellen, in benen ein fold)ez.

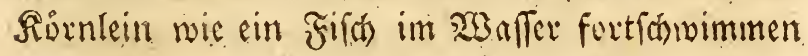
Fónnte, man muß an die engen Wâfe benfen, ano fid crinmern, sap man fich Die Circulation 


\section{LrV}

Der Crifte in ben feinften Srganen nidt ander alb Dutch Die Afuflofung in cinen uiberaus feinen

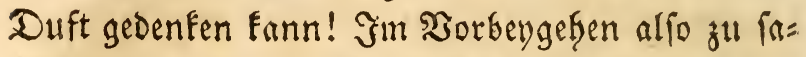

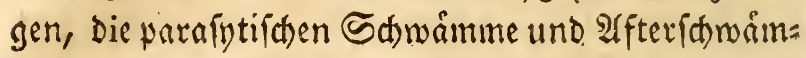
me, die alle Jahre auf ben Slạttern ber båus me und anderer Pfanzen erfheinen, módten moff? rher den zu gleider Zeit unit den Bewsidglen mie: Der auflebenden Jnfeften oder Der fpeciffifhen Reich= tigfeit igrex Sarmen, vermog weldter biefe hoch in die Suft aufferigen und nur bey biendittern von Dem Regen prácipitivt werben, Dab Vehiculum ifrer periobiften Waiederanbauung zu verdanken

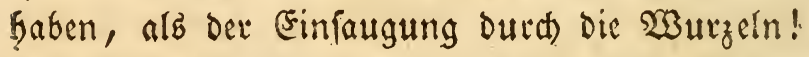
Man erinnere fidi) noch zum Ueberflü, daß die

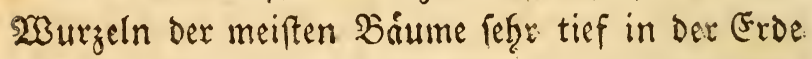
verborgen liegen, lind daß Dirfte, zu erfleiren, wie diefe Sdimammiamen einige Fuß tief Durch Den Froboden cindringen und

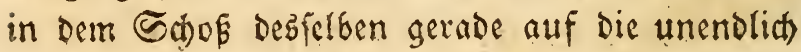
feinen Gaugfwithen Der 2 Surzeln zutreffen folten!

So wofl fier, bey der fueciellen Abgandlung

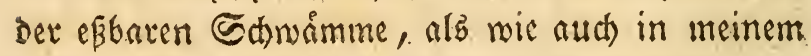

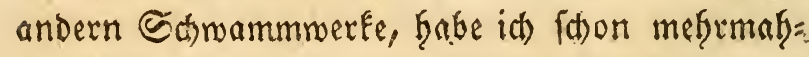
fen bie Unzuláffigeseit der meiften unferer nux pro= viforifá) eingefügrten Binttungen und $\mathfrak{A}$ ten Det

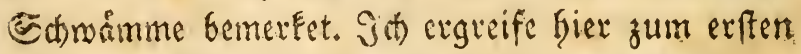
Diafil bie Bjelegenteit, meine Bjeranfen über cine 
Heffere Girunovefte Derfelben mit thrbefangenţeit Dentienigen vorjulegen, meldbe Dutab Dic Ectirfe ifres (jeifes uno burd ifye gropen Paturente niffe berufen find, bas siditeramt in folden sin: gelegenţeiten aแร่วuน์

Stre claffiften Theoretifer Der Botanif ftim= men in dem Sirundáąe úberein, daßj man die (Battungs = Eharaftere von Der Slutbe und Frutb), Die Egaraftere der Arten aber von ben bloßs we= getativen Sorganen ber Simadife Gerleiten fulle.

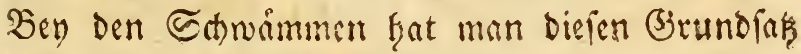
bistger nod fefre wenig befolgt, man fat bab eis

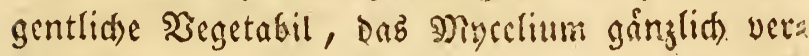
nackleffigt, man Gat bie Battungen von ber mei= fteng fefr zufirkigen und mandelbaxen Sieftalt deb Fncrrawimb, Die Arten aber von Deft Miodificas tionen berfilfen entlefret. Da wir nun aber, nach Demi, waz borauggegangen ift, Die wafres?a. tur Der Gednomme beffer fennen gelernt Gaben; to ergift fich, oas in Bufunft die Shnamm gattungen rut nath ben wefentlitben Sexfacheder. Geiten Des Encaxpiumb, bie Alten fingegen nach jenen bez Miycliums beftimmt netoen muffer. Der mefentliafte Theil bez Frudtfórperb ift ons Hymenium, Die Edurge, uno in feiner Sber= fliche die Staubblęzd)en und dic Srutforwer:

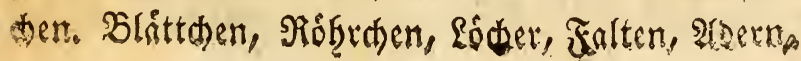




\section{LVI}

Galiken, Pfriemfáden, finootchen น. F. m., bas alles find nut foldte bildungen, die won gerin=

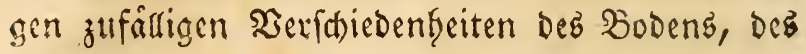
Etandortes, Der Witterung, Der Beferlfthaft u. D. gl. Serânderungen erleiden. Wenigftens dư: fen Diefe Eonformationen nur mit ciner viel gro $=$

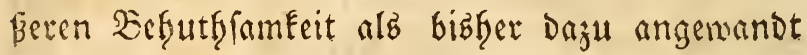

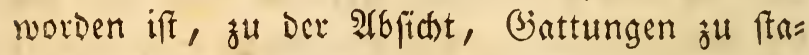
tuiren, benukt werden. Mian Finnte meines (5ra nôtens viel ficherer auf Die Cubftanz und den Sit des Symmeniums bauen, und fonach alle Battungen Der Schnaimme vor Der Şand auf folgende Dreb, nafmentlict): Lithothecium, $\mathrm{Hy}$ menot ecrum und Kaematothecium, weldbes ber) Perioon familien find, rebucien, of ich gleich Goffe, dap man in Der Folge micber viel mef̧ere, aber ganz andere, alb bie bisfyerigen Genera einfifferen werbe. Die Binttung Naema= tothecium begreift zwat foon bie fogenannten

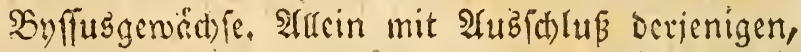

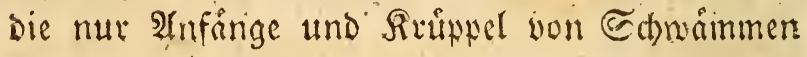
find, modten benn bod die lubrigen viefleidt noch) einft Der Familie ber naferen Sctinumme angefiorig befunden merben! Iath der Sot dez Shymeniums gibt einen braudearen Efarafter!

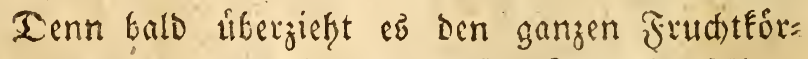
per, balb nut einen geniffen Igeil debfelben. 
Sald ift eg eingefthloffen, balo offen. (Ez unter= liegt zumeilen gemiffen Dermandlungen; es pros liferirt uno maihf mit Dem Perisarcium fort, nder bat auch wobl feine beftimmte Dimenfion

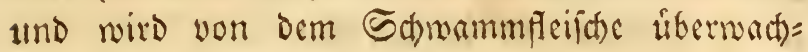
fen u. F. w.

Die Brutfirper (Sporulae) Der Crtwimme fonnten zumeilen nach ifger (Beftalt uno Farbe, cin anderes Mrafl fingegen nad) ifere Minge, nad) ifferm Etande, nadh ifrect $\mathfrak{2}$ (t) ju reifen und abzugethen bifinguirt werden.

Begen Die werfügrerifsen Pelorien faben nir Feinen andern G(d)u, als bic Scobachtung in ber Eultur uno Die 2̧ergleid)ung Der unver=

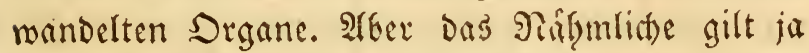
aud) won Den Welorien Der PGónerugamen!

Die Arten, babe ich gelagt, find nad) bem Mucelum (berfteft fich in Der 3 ufunft, wenn ein Miafl mefirere Brobachtungen Darüber ge= madit fern werden!) zu unterfheiden. Der Zeit= punt, in Dem man Diefes beobaditen und unter= fucten for, where nach meiner spreinung am be= ften Dann auszuwiffen, wenn es cben im bes griffe ftehet, Frudbtforwer augzutreifen, und nod effe, als fid) diefe entfalten. Die Merfmafl:, Dic man Datan finden Fonnte, lim \&eten zu un= teriferion, fonnten aflenfalls in Der Confiften 


\section{LVIII}

und Zähigkeit, in bein bemangiel mit fremben siorpern, in ber Entipinnung ber Fibern, in ber Snge, in Der 23erwandlung, in Der Farbe, in bem Beructe, in Der Nerbreitung, in Der Daucr, in ber Beftalt und in Den Sazmulafen úber bem Standorte gefucht und aubgewaifit werden. Die= fer $233 e g$ ift benn freylid fefre foldwer, aber er ift aud der einzige gur grúndliden Fenntnis dee Sd)wainme.

Unterdeffen bedienen wir ung aber zur $\mathfrak{A} u \xi s$ Gilfe Der bló erfunftelten Battungen und âten. Die Folgezeit witd ung (nofl nict)t mit Riefens (d)ritten!) zur Renntnis Der wirkficten uno nall: ren geleiten! Wir múfen unz indeffen begnugen, bie verifhicdenen Enwanmgebitbe, feven ez nun Irten oder blope Paturipiele fragmentarifón fen = nen zu leinen, und won zeit ju Beit neue $\mathfrak{A} u f=$

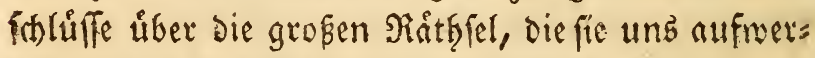

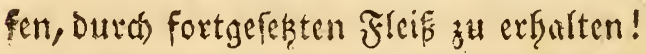

Unter ben neucren Futforen, welche in ber Miycologie den exfen Siang befraupten, frat Per= foon, ber exfte und youjuglidste derferben, folgen= De Binttungen feftgeferst; Deren mefentliche Shara É tere und Merfmưrigigeiten idh nun meinen Sefern in Umriffe Gier Darğufteren gedenfe.

I. Der Bitterfamamm (Clathrus) beftegt aus cinem eyrunden, fitellofen, gitterartig = fleifiogigem 
Sute. In Der Jugend ift diefer Fórper mit $e$ : ner meflid)t = Flebrichten Mlaffa angefưtet, im als ter fingegen ift ex ganz Gohl. Eine meiblithe

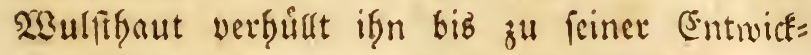
lung, uno bevor dicfe zerplakt, billet der Edhwamm ein weictes leberartiges (5y, wie die der folgenden

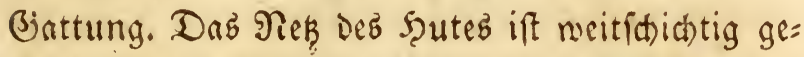
ftricft, und befteft nus dicken, in die Runde ju= fammen lnufenden, anaftomofirenden, faft cinno= berrothen Aeften, die mit Dem Hymenium befleidet

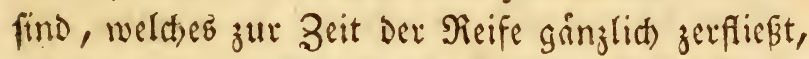
unb einen unertraglichen Sabaverófon (Beftant ver: breitet. Man Fennt bisher nur 2 squten bavon, Die in Den rorimften Provingen Des fúdicten (5u= ropa zu şaule find. Eie gefgoren ju den aubge= zeidneten Sabinfeiten, aber nidt zu den esbaren Ed)mammen. Einigermaken finden wir in Der $\mathfrak{B e r}=$

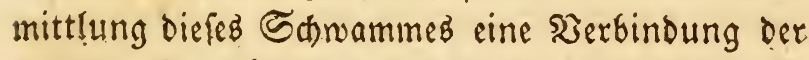

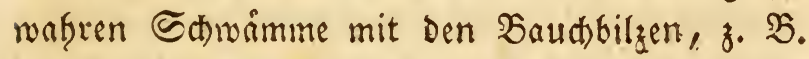
mit Lycoperdon bezeidnet, uno wivklid Gatten Sinnée und feine Beitgenoffen verfaciedene arten von Trichia, Arcyria, Stemonitis 4. 0. gl, mit Diefer. Battung veveinigt.

II. Der Bittrtidroamm (Phallus). Der Şut ift geftrunft auf leiner ganjen enformigen Dberfläche zediat; Das gallertartig = íleimichte Hymenium, getflefst in eine jaudse, in meldber oie Borutforn= 


\section{LX}

d)en fhwimmen. Sn ber Sugend ftect toer Edtwanm in ciner 28 ulfitaut, uno gleitht formlich sinem

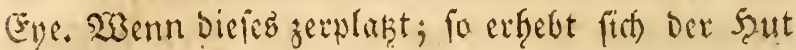
auf frinem Strunke mit fithtbater Eatherligfeit. Go, wie Der cingefchloffene Chwwamm alle Theile Des Eycs in Der Pachafimung Darftellet (f. meis ne Naturgefor. Der Defterr. Schwoimme. I. S. 94

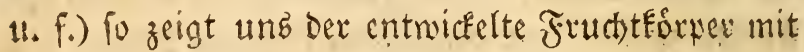
eince wirklic) benumberngnutroigen Ânalogie, bie

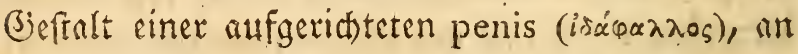
meldhit Die corpora cavernosa, bie Urethra, bie Glans penis, Dả̧ Orificium und fogar Daz̉ scrotum ein jedes an feinem Plake zu erfennen fino.

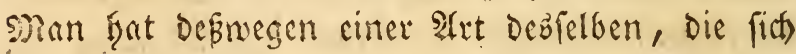

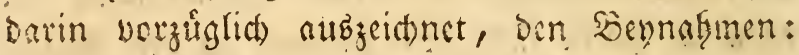
impudicus gegeben; und es iff auffallend, dấ die

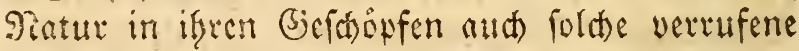
Dinge nadifnet uno fogat die Polngamie in geniffen Familien des (jewaid)äreidtes begưnftigt.

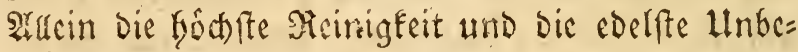
fungengeit Darf won Eeinem Inblide errótgen! Die S3itrde Der gatur ift weit ifer affe menishlide

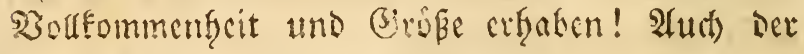
Unfduldigfe unter Den Gterbfiden ift Dod wes nigftens forfig, Dutci) thictifte Iricte crniedrigt zu nerden; Die Nature entefget fici niemaffen und Enn niemaflen entefiet merden. Darum bildet 
uno bronet fie mit Dex grofsen Frenfeit, und ife

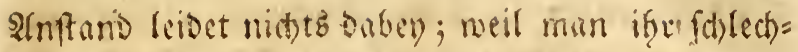
tcrdings feine andern, nls cole unto tadeflofe fichten jumutfen Fana. Sfyr gleithet einigermaß̧en Der menfonfreundide 53 undargt, ber mit wohl tfertiger Intarmtergigfeit ben leidenden fiorper

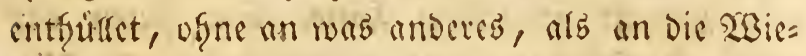
Derferfferlung feimer verlesten Befunofert ju ben,

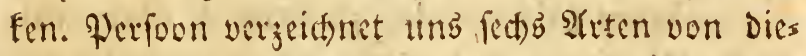
fer (jattung, die afle fur die Slrgeneyfunde witbtig zuleyn faseinen, und eine genauere Wrifung vers Dienten. Mian hat vordem Die Mrorct)elaten (Morchella) mit biefer (5)attung vercinigt, blop weil De: Şut Derfelben mit Dem Şute Des (Bia)tidhnammes

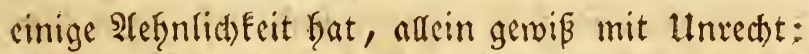
Denn Morchella Gat feine Volva, fein füffigez Hymenium uno die (Er.fdeinungen Der Mietamo: whole find in gochften (Sirade verfateden! Bum (sie:

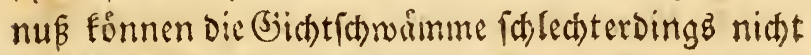
vermendet merden.

III. Der Gadeffwamm (Amanita). Şat ei= ne $\mathfrak{B}$ ulfthaut; einen fleiftigen, meiften Sheile getâfelten Scut, georángte, faft einformige famel= len, einen meiftens verlangerten Strunf und of =

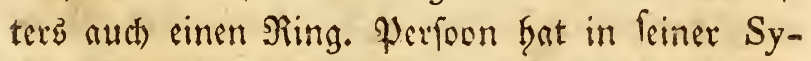
nopsis fungorum 16 2rten: aber es find feitfiet fibon wieder mefrele neue Siten entbect morben, 


\section{LXII}

3. 5. won Aftbertini und Echneinits. (5) gibt

3 atstgeilungen in biefer (sattung a) mit einer

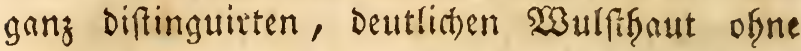
Sing; b) mit einer Deutlid)en 233 ulfffaut und ei= nem $\Re$ inge;c) mit einer undeutlicten, am Strunfe vernodfenen und fid) zerpfluckenden 230 ulftgaut, uno marzigt getifeltem Şute. Die Arten Der

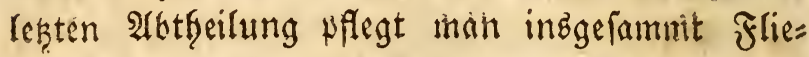

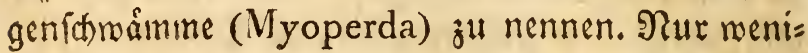

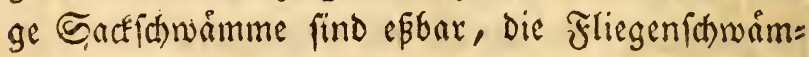
me aber ůberfaupt fúr giftig, zul falten. Die

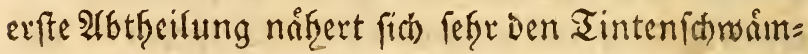
men, Coprinis, einer 2 bbtheilung ber Slatter=

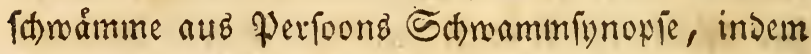
fie faft eben fo zait gebauct find, fefre enge (ges Draingte) Eamellen, einen 2 nfangs glocécenformigen f̧ut Gaken, und in eine Jaudhe zerflesßen. In ber 2ten Ibtficilung find mefreve, wie z. 20. Der Rai= ferling, epbar. Dods wirb audi) die Amanita in-

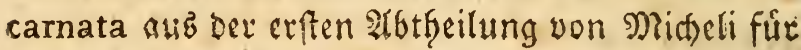
einen Edwamm angegeben, welcher man in Jtas lien ofgne গachtteil veripeifet. Dic Gattung Amanita (fo mie wir fie fier nefimen!) ift offenbar nux eine erfunftelte; Denn auber Dem $\mathfrak{B}_{\text {ulfte }}$ ift fie

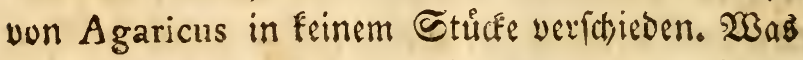
nun aber biefez Srgan betrifft; fo finden wir in aud in Den übrigen Agaricis f̧ånfige Spusen un๖ 
Hebriginge bavon. Bey Den erftgenannten Coprinis ift fie an vielen 2 liten in Dem erften $\mathfrak{A l}=$ ter gar nicht zul verfennen, meffece Agarici

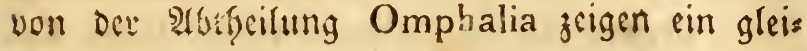
()

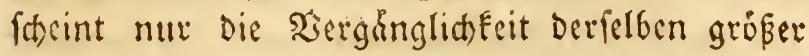
und Die হerwachiung an Den Strunt ungefáfer wie bey ben friegenfómmaimmen fefter z" feyn. Şicr ift nod) ju bemerken, daf La Mark nach Dem Seypipiele des Dillenius, und F̧aller unter A manita ganz waz anders verftef̧, alz ben Sack=

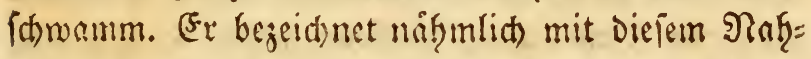
men alle jene Areten bes blatterfhwammes (Agaricus), weldte einen fleifdigen frut und einen Strunf Gaben (ङ. Encyclopśdie méthodique; Botan. T. I. p. 104 et 694). Unter Agaricus fingegen verfteft et die perfoonfden $\mathfrak{A}$ iten von Boletus, Daedalea, mef̧rere Merulios + Sistotremata u. D. gl.

IV. Der Slintterfdomamm (Agaricus), Der Şut Gat Blåtter, oder Slåttáen (Lamellae), D. i. aus einer Dupplicatur des Şymeniums beffe= bende und voin Eentrum nad Dem Rande Deg Şutes bin @irafilenformig auslaufende, fdarf= randigte Sappen. Unberectinet Der neueren $\mathfrak{A}$ rten und Der ungeffeuern Menge Der zmeifelfaften Onrietåten, Dic vieuritht (in Der Mganier, mie 
mant bisher Die afren beftimint fat, zu verfate= ren!) nod Dem großsten Theile nach) für eigene sireten in Der Folge ausgegeben werden Durften,

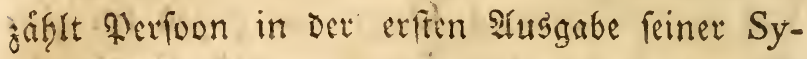
nopsis 447 Species. Fis find Die Amanitae Def

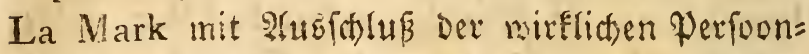
fthen Amanita wno mit Snbegriff der meiften $\mathfrak{A} \mathfrak{r}=$

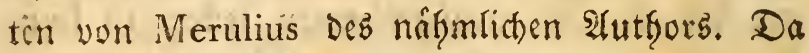

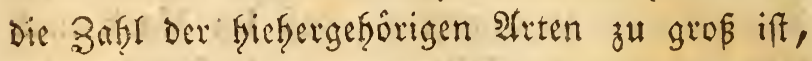

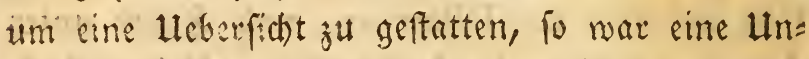
terabtfeilung in gemiffe Familien goochit nothwerk Dig, bie Denn audt Perfoon wirefict) mit fefre vielem (j)fucte genacht fyat. গut an bie Stefle Der weiteren llntertfeilungen ber Familien feltfft waren mandt)mafl nod) andere und forarfer af. gejectonete jul minichen. Die fo eben erwähnten Familien Der Blåtterf(bnaimme wåren Dann nat) Werfoons Synopsis folgente:

A. Lepiota (ভticfelfdmamm). Die Ramel: len find etwes trodfen, unwandelbar, Der StrunE

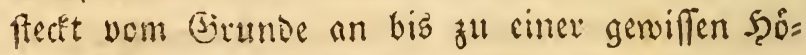
be in einer feft verwathenen $\mathfrak{S}_{2} \mathfrak{a}$ t, bie im jủn=

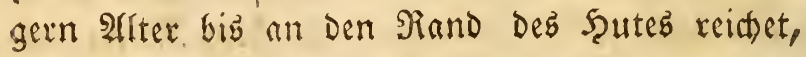
und alfo die Ramellen ve:fhlüft. Diefe Sraut reist fid) nachfger um und um von Dem Şute lob, und bitset ben Ring, welcher bey einigen Irten fefge lange dauert, bey andern fingegen 
fammit Der Decfe dez Strunfez fef̧r bald ver: mefet und veridjwindet. Agaricus collinitus, meynt Perjoon, fornnte in Der Jugend leicht für ei= ne Lepiota gefalten werden, ba er boch in reifes rem 2 (ltex fid) alb eine beutlidfe Cortinaria zeiget. Dieje Familie enthålt mefreve eşare Sanoimme, aber aud meffrere verdådtige. Sie fraben daz

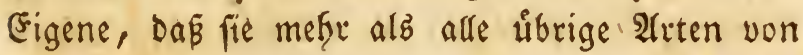
Agaricus geneigt find, jede $\mathfrak{A}$ trt furr fith in Dec Bróke ftarf zu Differiren.

B. Cortinaria (ङdeleyerfhmamm). Der Şut ift meifteng fleifdsig, Die Eamellen an Der Bafib eingeidnitten, genoógntict mit bem Srute und Strunf von einerley Farbe, merden jedoch an Ende mefir oder meniger zimmetfarbig; Der Strunf,

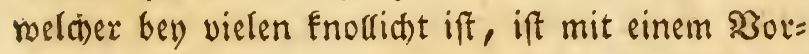
hang verfefen, ber fich faserergin in ein Spinne= rebenartiges Giefáfer zerpflucete. Die Unterthei= lungen diefer Familie ferzt Perfoon nad) Der Farbe feft. Âtein Diefe ift bier nid)t ganz to zuverleffig,

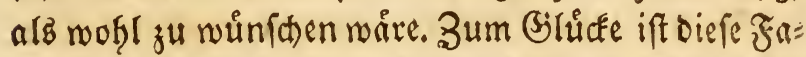
milie nod) nicht won ben allergróßten! $\mathfrak{X a b}_{\text {aber }}$ bier vorgůglid viele Schmierigkeiten fervor bringt,

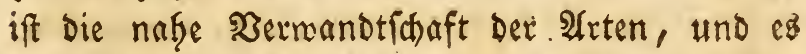
fheint, Daß in ber Folge mohl fefte viele Davon rebucirt, und fưr blope Spielarten erflät wer:

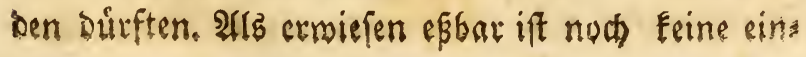


Jige von Den 53 Arten Diefer Familie befannt

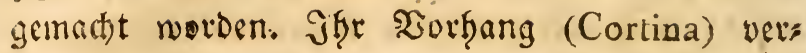
fdlügt in Der Sugend die Eamellen, und oft bleiben an Dem geoffneten Fiande Dez Şutes nud Sxuren beb̧felben in Eleinen Fezen und Fafern ůbrig.

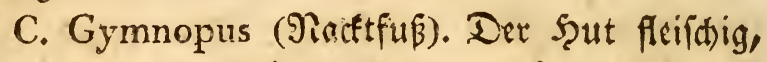
wo̊llig rund, geroólbt, Die gleid)farbigen Samels len vertrodfnend; Der Eentralftrunt ofine Sor= fang und ofne अing, alfo nackt. Sm Stande ver Jugend find bie Spute diefer Samammarten am Manie fo ftarf eingerollt, Daß Einrodung bie jungen Sumellen to gut beidusht werden, wie bey ben Arten der yorigen beyden Frnmilien, Durd) Den Ring und Durd) Den $20 r=$ frang. Dicfe Frmilie ift bie itarkfte von allen, und entfrailt nidt) menigev als 138 2atten, die freylich) woght Dem grópten Ffeile nad), nur Mo= Dificationen und 2 barten zu Feyn fitheinen, aber Dowh in ber gegennoritigen Mlanier als Arten ges trennt und unterfhieden werden musten. Die viclen von Perfoon feibft nafinfaft gemadeten Siten andever familien, als wie Ag. Mycena roseus, Ag. Myc. strobilinus, Ag. Cortinaria mudes, Ag. Omphalia adustus und felfeft ande: xe aus ber nafimlichen Familie, die wie Ag. fertitis, grandis etc. in andern, als benfelben 9 fo 
theiltrigen gefudtet werben burften, in welden ex fie

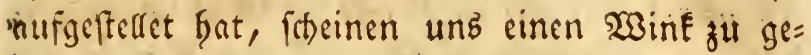

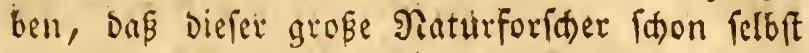
Die nafe anverwandtadaft gewiffer aud in ber Synopsis entfernter Sdirammarten gefüflt Gabe, und Das unicre obige Befrauptung von ber Erfchei=

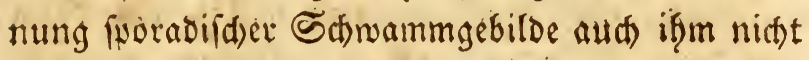
ganz ungeafindet aufifoffen bưrfte. (FE ift no(t)

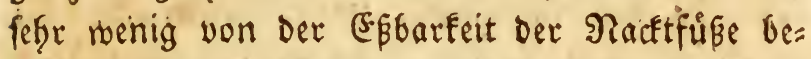
fannt, Denntod idseint ez, Das gerade in biefer Fantilie Die meiften eßbaren $\mathfrak{A r t e n}$ Der Edwånme aufgenofimen fenn Dưrften. (ङz fommt alfo nut nod)

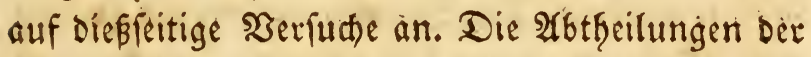
gegenwertigen Familie in Eleinere Eectionen fino von Derioon ebenfalas nad) Den Farben benannt worden; aber fie find aud eben fo unfider mie bies felben ber vorigen.

D. Mycena (Nagelf(h)amun); Zarte; fleine

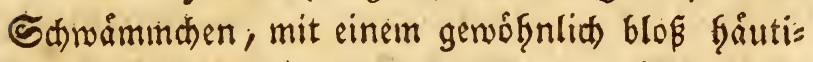
gen, geftreiften, burd)f(t)einenden, gewobloten und aub̧baurenden Şute, eintrodfnenden gleidjfatbigen Eametren, und einem meiftens boflen, verlanget: tèn, nacten StrunE. Die 39 Arten biefet Fami=

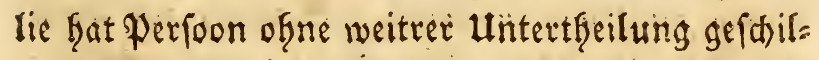

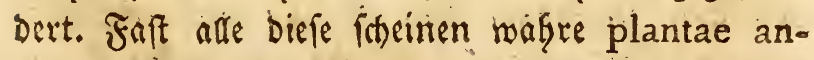
nuae (Sommergerwådfé) zu feyn. Sie bauern.

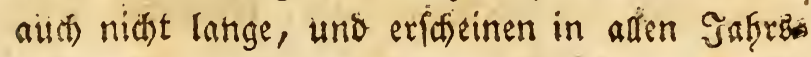

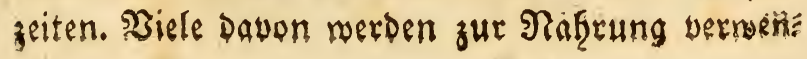




\section{LXVIII}

Det und mit bom gewófnfichen epbraten Ragete formamme verwechfelt. $\mathfrak{B e n n}$ fie faul uno verbors: ben find, mogen fie bie siefundferit verderben, bee fonders menn man fie im Hefermanke genießzet.

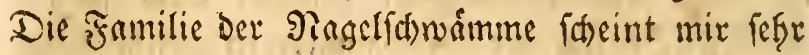
nenig mafie ateten, fondern nur Spielarten $j^{u}$ entfialten, aber biefe wenigen mögen fid bann

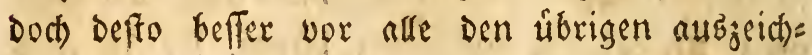
nen. In ben innerliden Eigenidhaften modten

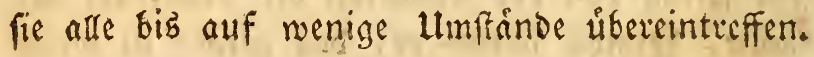

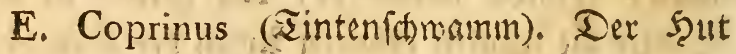
bautig, verweslid), ober etwas fleifthig uno am Ende jerberftend. Die Samellen nerben am \$unfte

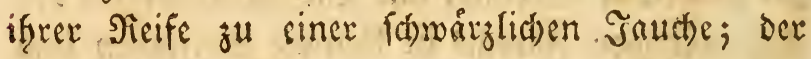
Strunf if meiflid mit oder ofne Ring. Die fief̧er gefforigen Ectswaimme wathfen alle auf fefte unicinen Srten, z. $\mathfrak{B}$. auf faulen brettern, Dinger u. o. glo; fie find von feftr verfotiedener Briópe, Gaben aber faft nur 2 frauptformen in Der Bjeftalt. Die exfeen baben cinen Bjlocfenfor: migen, am Etrunfe faft anliegenden Şut, eine weise Sirundfarbe, in Der exften Sugend rofen= rothe lamellen, bie aber an Ende ganz fofle fdomarz merden uno vorrig zerfiesen, mo Dann

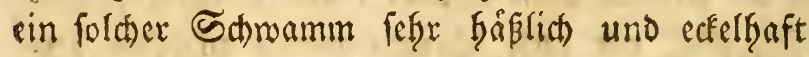
ausfief̧t. Die Der 2ten 2atheilung faben mebre

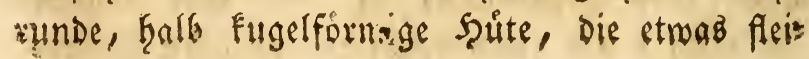




\section{LXIX}

pithig fino. Die immeften werden in ber lesten Rebensperiode flectict), angeloffen und gleict) fam

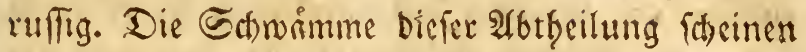
faft dex folgenoen Familie anzugefieren. In Pex: foons Sobuamminnople befinden fict 41 2reten Tintenfonamme. Afle find für giftig zu f̧alten,

F. Pratella (2rBandelfchnamm). Der Sout ift fleifdig ober aud faft brutig, glatt, Dauer baft; fino feine sameden etros angeloffen, fo merden fie zulezt forwarg und fdomukig, find fie aber einfarbig und nux etwas mafferig, fo nex: Den fie Durch das Earmenpulver blok verbuftert. Mandamafl fat Der Etrunf einen Ring, mand): mafil auch Eeinen. Unter Den 24 arten, Die in Diefer Familie bey Perfoon vorfommen, gibt el. sinige ebrare uno vorzuglich bie Efammignonz. Die meiften aber foreinen ungeluno ju feyn. $\mathfrak{X B}_{e}=$ nigftens forte man fie nut in ifrer exften $\Im_{u}$ geno (im jungfirulicisen 3uftande) verfpeijen. \$er foon nuift die Frage nuf, of nicht Ag. Lepiota haematospermus und Ag. Cortinaria viscidus et Gomphus mit mefrerem गist) te fieffer gezafilt merden forten? Die 2 Bandelfatroamme Gaben perennicende Mycelia.

G. Eactifluus (Brọtling). Dex Sut ift flei: foig, faft bey allen vertieft. Die Ramellen trâ

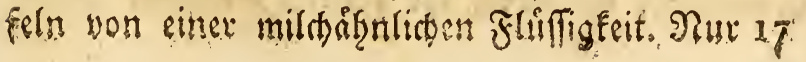




\section{$\mathrm{LXX}$}

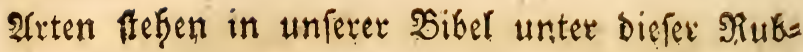
rife. Sie find epbar, in fo fern ifgre Mrild nictst

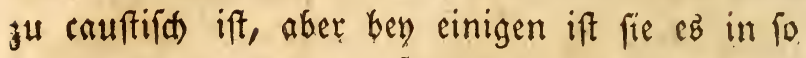

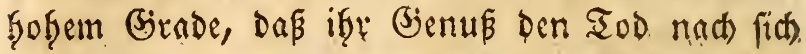

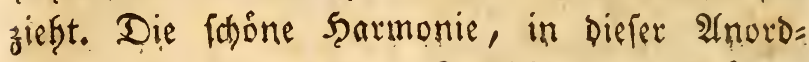
nung Der Perfoonf́ten Familien Der Slåtters fanomme ift fier befonders auffallend. Şir bas ben Den Hebergang yon Coprinus zur Pratella bemerfet. Siele yon Den Âten differ Familie find fdon Dild triefend, nur nicht in fo fof fem. Girabe, wie vie Drattinge. Diefe Lactiflui end: (id) nifigern fich and fiton mieber Durch bie gee

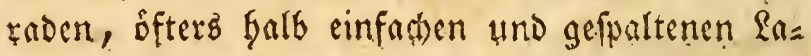
mellen den Trublingen der nådfffolgenden zafine, uno greifen fogar auch fdon mciter vor, naifumlich Durd) Den genabelten niebrigen squt, bis an den

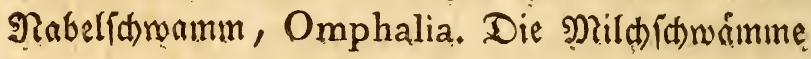
Gaben alle untereinander eine fefre nafe $\mathfrak{\text { Sernand: }}$ fdaft. Dennod, da fie beftimmt an genviffen Dis. tẹn zu Şaufe find, uno alle jabre exfdeinen,

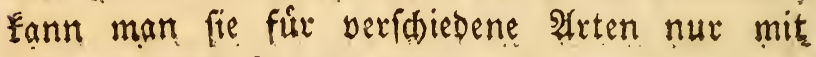
einiges (Einforónkung Galten.

H. Russula (Tánbling). Der fleifhige Şut ift genosf̧nlith niedrig, die eintrodfnenden \&amel Jen 'fino gleidlang. Der nacte Strune ift ge= moffntich meif. Die Tarublinge find gefáfrerlicte

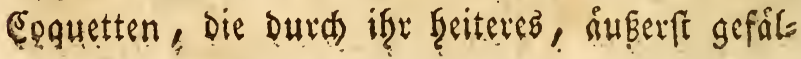




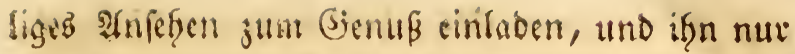
jul oft mit dem Sode bezalilen liffen. Dennod werden fie bafufig weriseifet, befonders die grau= grunlicten, die man (jitülinge oder (jivemlinge nenmet. Eie (t)cinen Durch. Einnutglung in Den exften 2 Begen diefe lethalen 23 ivfungen Gerworgu= bringen. Daxum mogen dic jungen am wenigften fat)aden, wenn man fie gut reinigt, 233 welanfaks und fameffen abjonoert, milo fie lange genug auf

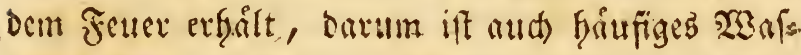
fertrinfer und Ealte, raffe Hmfáláge das befte fifecifife Begenmittel. Perfoon bat 24 aten ba won, und theilt fie nad) Den Farben cin.

I. Omphalia (Nabelfóncumm). Dec Fefir.

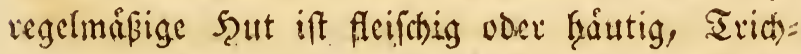
terformig oder genabelt, bie uagleich langen Safts

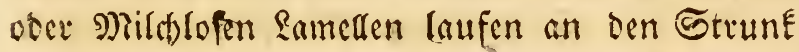
Gerab. Der Eentralftunf ift nad. Die Scauptaba.

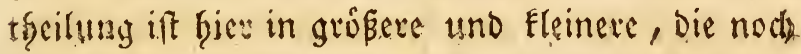
meitete. Intertgeifung nach den Farben. Son berk 50 Arten diefer gropen Familie ift noct) feine als epbar befennt geworden. Dod fidsinen mefreve Develben misflia genubber zu feyn. Mandse vor

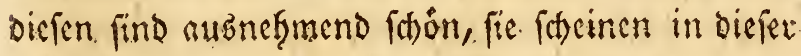
Sorde bas zu feyn, was die Papageyen unter ben Rogeln, die Sametterlinge untex oen Șnecten, 


\section{LXXII}

und die filiaceen unter ben phonogamifhen bies wådien find.

K. Pleuropus (Sdhwimmer). Der Şut ift fleiffig, nicorig, folef, ganz= sder fralbrund, Der Etrune nacét, excentrifo, dur Seite einges fügt oder gar nidst vorfanden. Diés find faft Inuter Parainten Dex Båme, an Denen einige Derfertben oft in gropem biedrange hervorbechen, uno fich Dartziegelformig bedecken. Ëb gibt un= ter Der Biattung Amanita, und unter der Fa: milie von Lepiota mefreere, die zll Der gegen=

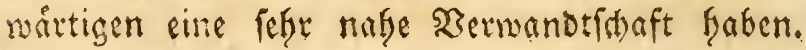

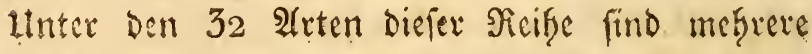
effar, now feine-aber giftig befunoen worden. Dod) fino vicle Daven ganj unfatmactifaft, les Dermetig uno trocken.

V. Der 2 deridniamm (Merulius). Der Şut ift fleifchig oder friutig, sie Schủrge aberiat, mit oberfladigen, aufgedunferien Sroern ober Fals ten. Diep (5attung umfast fefre ungleichar=

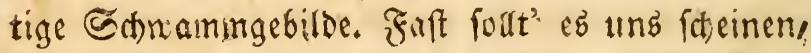

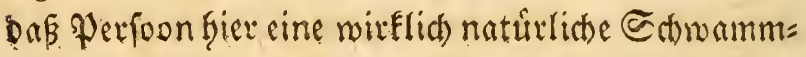
gattung Gabe bilden wollen, weel er bie form Der Frudtefforper babey meniger, alz bey irgend eis ner andern, in 2 rbficht genoismen, und feine"

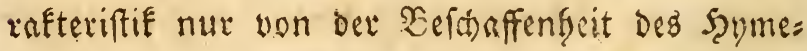

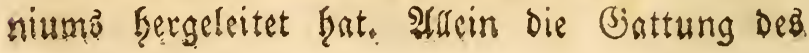




\section{LXXIII}

2doerfonammes ift beffen ungeaditet nod immer zul gefunftelt und zu gemengt. Dafhin fdeint auct) Fton Die Erfafirung zu Deuten, Dak es fo fatmer ift, bie Braingen jnifhen diefer und jenen von Agaricus, Daedalea, Sistotrema, Thelephora, Helvella uno Peziza zuf finden. Dic 9 atbei lung der fiex aufgenofgmenen 25 Arten in 3 Familien war um fo nótfiger, Da fich biefe wirk: lich fo refentlich unterfacioen, $D_{a \tilde{\beta}}$ fie in bem Bielfe der Methode jebe fur fich zu einer eige= nen Gjattung erfoben zu nerden verdienten. In Der That aber fibeinen Die Merulii ber 2. und 3. arbtheilung nur Monftrofitaten, oder Pelorien zu fenn; bie exfe fingegen fid) ganz befonders

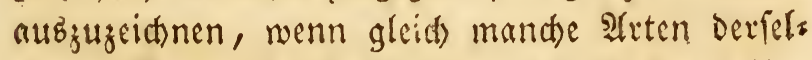

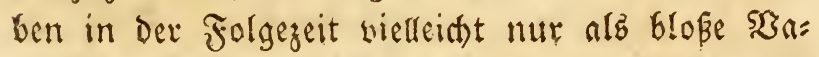
vietaten vereinigt merden Durften. La Mark vers fteft unter Merulius die Familie Pleuropus Der worigen Binttung und unfer Merulius Cantharellus ift bey ifgm eine eigene (jattung, die Can-

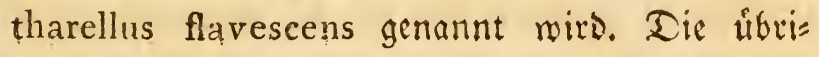
gen-Arten unferes Merulius find bey $\mathrm{La}$ Mark theils unter feinem Merulius, theil' un= ter Agaricus in feiner bedeutung zul fucten. Sinne, Juffieu น. D. glo Gaben noch Esimen Merulius, fondern fteren die Arten diefer Giattung unter bie Fafgne von Agaricus und Boletus. 


\section{LXXIV}

Saller ift bet Etifter Derfelben. Die oben ers

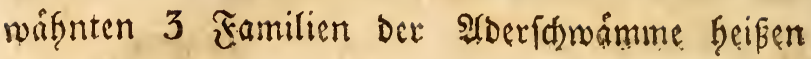
nach \$erfoon :

A. Cantharellus (Pfefferting, Rôtţling). Mit cinem vôflig runden, meiftens becher = pper Trichterformigen Şute, und. cinem Eentralftrunf. Einige $\mathfrak{A}$ rten von Der Framilie Omphalia, als z̧. $\mathfrak{b}$. Agaricus involutus, gilvus,' squammulosus, lobatus, cochleatus u. D. gl. haben fieffer eine fefte mefentliche beziefyung. Fine 2 te anonymifitse Ultterabtheilung Diefer Frmilie, die man feffre leidt überfefen fann, waßk gar nicht ju der gegésenen Definition und entfialt lauter zarte, oft ftrune:

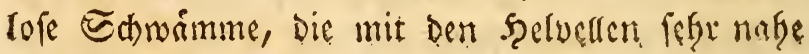
virwand find.

B. Serpula (Ţfrẹnenfomamm.) Dicfée 4 Er(t)mammarten wưrde ich) liefer mit Daedalea und Sistotrema zufammen in eine Gattung ver=

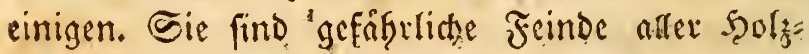
fabrifate, und baben falon offterb ganze Şrufer ju (jirunde gerichtet. S(ch) faf die Şauptart, sen Merulius destruens $P$. in Dem vormafgligen Bies. båude bez Giefigen Univerfitåţgarten Gerríten, und ez mußte eingeriffen merben. Ifud traf ids

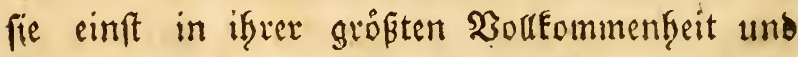
\$erbreitung in ciner 2f(penfưtte, auf Dcm Serge

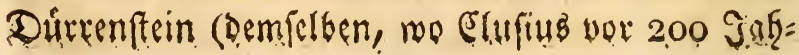


ren geweien, und deffen Bipfel feit jener Seit, meineb 23 iffens nod) von Eermem andern Bota nifer erftiegen worden!) in ciner betraintlid)en Sobfe auf dex fogenannten Sermalpe, wo ex bas ganje Biebrube fo iefir foton angegriffen batte, Dap ich bey jedem (S)ritte in cin aubgefreflenes Roch Des Fubbodens verfant. Rangz Der ganjen

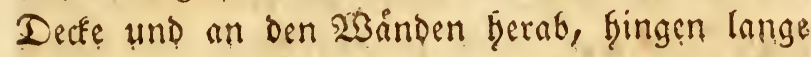
Botouren nom Ectmamme mit fancerocifen, bict angeloffenen, faft freifichigen Randern, die gegen

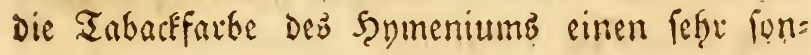
Derbaren, ecfelgaften Contraft Darftellen, welchers id) męis nicht Durd) was fire cine SDeenaflocia: tion in init ungeffint eben benfelben afbftisu, mie der anblicf yon fefr fetten Epinnen, Rro: ten uno Eipechfen ermecte, Das $\mathfrak{B a f f e r}$ tropfte

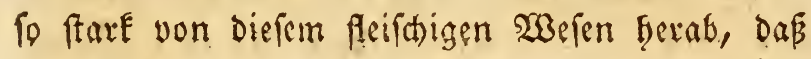

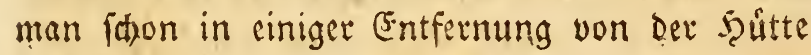
Dieß Stilliçidium vernefluen fonnte. (jeruch max noch das alferunangenefomfte, was ic) Dawon empfend. Fr gliech geniffer Mapen Dem (jexud) einer Sodtengruft, es mar ein muffender, etwas s)laufeartiger, mepfitiliber (Bis= ftank von foldoer seftigfeit, Daß id) nid begrei= fen Fann, wie ifgn die Menifhen, die ba rophn= ten und fadiefen, extragen fonnten, ofne frant

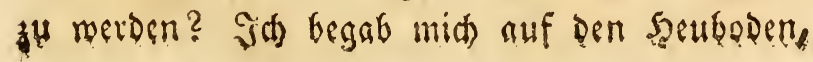




\section{LXXVI}

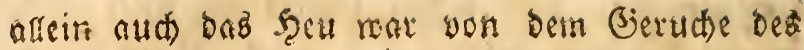
Edjrammes verpeftet, uno obgleich ber $23 i n d$ nur gelinde max, fo bemerfte id Dod bie ganze Racht bindurd mit groper Unwufe das Sirachen

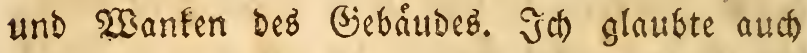
in Der Dunfelfat einiges. Phospforefcicen baran bemerft zu baben; wenn eb nicht etwa cine Saufdung wax, denn es gab da fo viel whoz: whorefcirendes Sgolz, Daßj ich mich an leinem 2 nt blicf fư die : voorig entichábigen fonnte.

C. Gomphus (Şafenofhrlein). Sin Feulen= formiger Sdnormm, mit fefre fcinen 2 doen an ben Eeiten. Diefar Eshamm, welther von Eamie= Del und $\mathfrak{B}$ ulfen in 9 thbildungen und Seforeitun= gen befanm gentact murde, ift ein fefer munder= bares und nuffullender Saftard. Ere ift nábmlich feiner ganzen Geftalt macb) eine Clavaria, zu= weiren, ment er nafmlich cinen unvollfomme men Şut Gernugtreibt, eine Helvella, und nach feiner Eeitenbedcfung mit der Sdurize ein Mie= ruliub, Dex mofl aud) ber Daedalea fid $n \mathfrak{n}=$ Gert. Scine Subftan im reifen Alter ift fork, artig, Die Farbe rathleaun oder violett. Dben ift ex ganz eben abgefonitten uno gleich fam ent= Samptet. Man Eonnte fich vielleidst vorftelfen, páp cin Brutforn von eimer Dacdalea, wann sद 
in Der (Frbe zum ficimen gifommen, foldte Futht: forper fervorbringe, Deren fonft immer angerwadz:

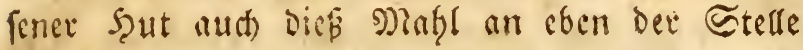
flach) und ofine bedecfung verblicben, an ber ex fich fonft anzufeften pfleget, oa er Dicß̧ MTafgl Eci= nen Giegenftand getroffen, und man fönnte von Diefer Soec auggefgend, Den Durchgang Des Şutez in Den Etrunf und cine Menge anderet Fradaei nungen in ber Siloung und Entroidelung Det Edhråmme begreifen und exffiren.

VI. Der \&abyrintffithmanm (Daedalea). Det Galbirte Seret= oder Forfartige Şut ift auf Der Un=

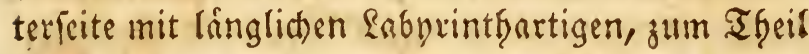
Rocherformigen Sertiefungen Durchbrochen. Die (5jattungen Daedalea und Sistotrema laffen fid) mofl in feinem reefen Bfyrafter yon einanber un= terfheiben. Beyde find in iffer Jugend Boleti.

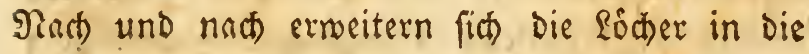
fange nad) allen Sidt)tungen, wie die Laufgråben bey einer Belagerung. Alm Ende gleict)en einige

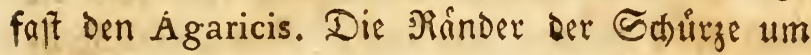
Diefe (Sjange Gerum, nerden naturlicher $S_{3}$ eife eben fo verfdiebentlich auggedefinet, und weldbe derfel= ben macbien, befonders am Grundftuce in ver= (w)iedene faft F̧yonentige Fesen und Spiken auz. Perfoon f̧at nur 5 arten von Daedalea: atrein gift mofgl nom mef̧rere, und sinige find won 
fold)er Shonfigeit, bap man nidft fo leidt etwas gleichez unter ben phónerogamen finden mag.

VII. Der Gódterfthwamm (Boletus). Der

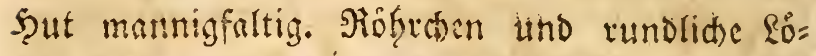

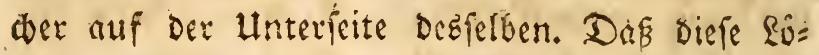
ther cine ungerthlugte Miunoung Gaben follen,

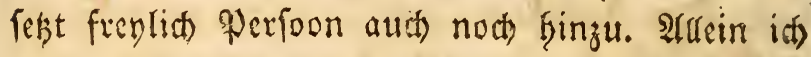
finde, daf alle Boleti, merin fie alt werben, nur mefre oder weniger, befonders in Der Naffe Des Cetrunfes oder des Birundftúfés, momit fie

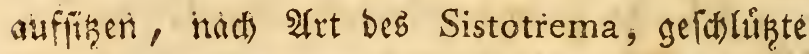
Mưndungen befommen! In \$erfoons Sdhwamm= finowe finden fidl 93 Irten von Diefer (Sattung aufgersfinen. Seil La Mark beipt diefe (jattung nad) Den Stten Agaricus, und mernn ber Frey= Serr won Sqquin in I. SDes. Feiner Flora Aus-

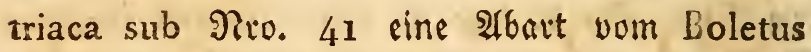
lucidus P: Agaricus Pseudoboletus nannte; fo muß man dieß nidet fư cin 2 erefefen, fon

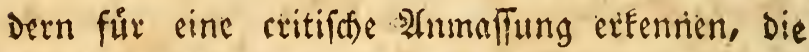

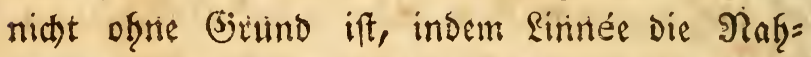
men Agaricus uno Boletus gegen ben Bebraud und gegen die Brundrakne der Philosophia Botanica verwedfelt b̧at. Dod feut ju Tage find nun cinmafl bie Sinneanifoen Nafymen alfge= mein angenofimen, und wir miffer fie berbes Eqalten, um Esine Bernirtung zu ftiften. Hnjer 
Elaffieer theilt biefe Bnttung in folgende Fami= liin ab.

A. Suilli (Bilflingi). Der Sput ift gepolftert

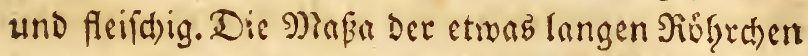

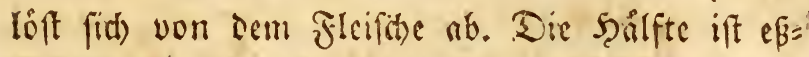
bav, bie Şrffte ift giftig. Jur giftig ober Dod verderdtig find hale jene zu Falten, Die nach bem

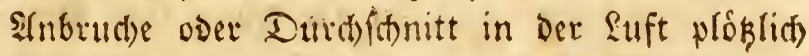
ihe neife doer gelbe Girundfarbe in Slau, Bivin oder Slenfarbe vernandern. Fie find eine vorgugs lide Bierde Der $2 \mathfrak{s} a$ lder.

B. Diatrepi (Etid) (d) wimmc). Det Shut if fleifdig, Rederartig oder nodi) ofter Rorfirtig: Die

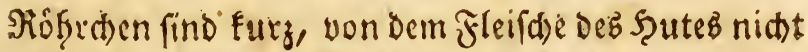
abgefonorrt. Dieß fino bie eigentliden normalen BoJeti; Denn die Suilli módtèn wegen ber Robgebung Der Sódermaffa wohl cher eine befondere battung aus̀maden? Da die Familie fefre groß ift; fo fzat

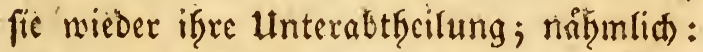

a) mit einem ganz runben Sçute und einem fenf: reaten centralen, oder ercentrifaen Strunfe;

b) init einem f̧albirten Şute uno sinem ธeio tenftrunfe;

c) mit einem falbirten Gtrunflofen, o. is auff fikenden Seute.

In ber lekten diefex Ünterabtheilungen fom: men einige arten vor, bis zufammengenofimen eif 
ne eigene Gattung ausmaden bưften. Dergleident waien Der Boletus fomentarius, igniarius u. f. w. nafgmlich, bic Zunderichnaimme! Die annuellen Sunderid) waimme betrad)te id alz corrumpirte 23 af ferfüdtige 2Garten!

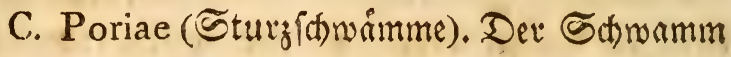
ift unigenendet, D. ift: auf feiner Dberfeite Siches rict)t. Die meiften Gaben faft gar feinen .̧ut. Per= foon fheint biefe und dis folgenden Familien faft fir eigcne (jattungen zu Galter, und id) waive allerding Damit einverftanden.

D. Polyporus (Narfenidiwamm). Det Sonwamm theiit ficí) in Sapwenfơrmige $\mathfrak{A}_{\text {efte, }}$ bie son allen Geiten mit ziemlich groben Söchern vers feffen find, und gleidffam Blatternarbidt auşefenen. Die einzige Giefger gefforige $\mathfrak{A} \mathfrak{\text { th }}$, Bolramosus, mách nut in Sergwerfen an ben faulenden Balferr.

E. Fistulina (Flcifthiftramm). Faft mócht?

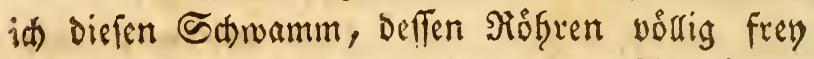
fino, aub Dem ganzen biebietfe der Sdynaimme Geraugiesípen, und ifn an eine andere Stelfe un= ter die Pgónogamen $\mathfrak{z}^{4}$ Cynomorium bringen. Die Reld)e jenes anfơngliden zuftandes fdeinen mirflict) Blumen zu fenn. Daz Mief̧rere fievon Lfe man in Dem $2 \mathfrak{B}$ erfe felbft sub Lit. V. शRro. 21 , 
LXXXI

VIII. Der \&̊d)erzaf̨nfdruamm (Sistotrema P.) Dex Şut ift mannigfaltig; vie Schưrge ift anfangz rundlódericht, miro aber nadber in un= formliche Zånne jexfhlükt. Diefe Bjattung miro wafiridbeinlich in ber Folge wieder eingefgen můffen. Die Saftarden, Monftrofitaiten und Pelorien $a b=$

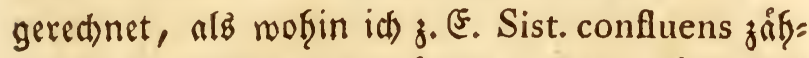
le; fo fdeinen mir bie ůbrigen Arten fämmtlid niđ)tż weiter alz Boleti uno Daedaleae zu feyn. Sie Synopsis entḩâlt 12 Arten Dawon. Perfoon fayt, fie madien einen Hebergang yom Boletus zum Hydnum. Man muß aber nid)t allez Heber: gang nennen, was einjelne Aefrnlid)feiten bars

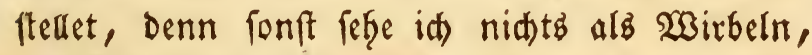
unter Denen Dev serftand exliegt!

IX. Der Eradidfarmamm (Hydnum.) Det Şut ift mannigfaltig, Die Sdưrge Jgelförmig D. i. in gange Pfriemartige Zånne geformt. Ein Stachel

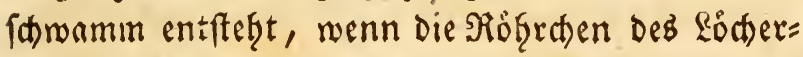
farmammes fich ganj von einander abjondern und an ber Spize gefaloffen find. J̈m Norbengefen

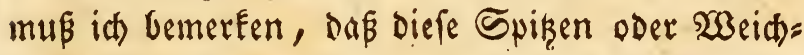
facheln nidht immex ganz einfads fondern viel mefre fef̧r oft in 2,3 und meţrere Spikgen zerţeilt find! Die Stahelfanamme faseinen nidsts meiter

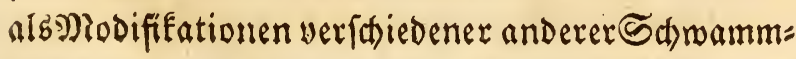
gattungen z" feyn, vorguglid Der Boleten uno 


\section{LXXXII}

Ber Slavarien. Son biefer Baitung enthait Per= foonz Synopsis 26 Itrten, Die ex in folgende Dronungen ahtfeilet:

a.) geftrunfte mit einem vơrlig runben, Deer mofhl aud) etwaz̉ aughgeidtweiftem f̧ute.

Diefe fommen ben Soleten am neidfifen. Mef̧= reve fino esbar und einige won augnefimender

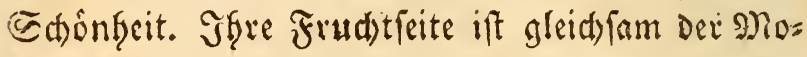
Del, in Den die Diatrepi geguffen morden: denn bey ifgnen ift gernde ba Reere wo die boleten yoa find und গ্sólle no diefe leet find.

b.) mit einem Ģalbirten meifteng Gtrunflofen oder nut magvedt geftrunftem Şute.

In biefer atgtfeilung Gat \$erfoon auth Hydnum Erinaceus, Der nid)t Gieffer gefforet, fon= Dern sielmefir eine eigene (Sattung ausmad)t, Die bey La Mark und Jussieu Hericius (Urchin) geift. (5s if sigentlich zu fagen ein Satmamm, Der einen Strunk, aber feinen Şut Ģaf. Statt Des Şutes jertheilt fich der Strun unmittelbar in eine grope Menge von Ieften, Die aber zu Grä fig und zu gedraingt find, als Dap fie aušnadjen fönnten und Dafer Den Pfriemfádden bez Stactel= (d) wammez åf̧nlid) verbleiben.

c.) $\mathfrak{B}$ loß außgegoffen, verfeftrt d. i. oberf̧alb. Jgelformig mit runden Stadgeln of ne allens Şut. 
Dieß finto alfo die Biegenftưfe oder die Sius= formen uon Poria! Perfoon foreint fie fur eine cigene bjattung zu falten, und nennt fie Odontia.

d.) Dhne aller Sinalogie cinez Şutę̧, ein Reulenfatwammformiger fiórper, neldser won allen Eciten mit Stadeln befest ift.

Aut) Diefe $\mathfrak{A}$ btfeilung Diftinguirt Perfoon mit einex $\mathfrak{A}$ (นz子eichnung und nennt fie Hericium, weldtes wohl zu merken ift, damit man fids nicht mit Dem Hericius der franzofen confundice. Die menigen fief̧er gef̧origen arten fino mirflich $C_{0}=$ rallenformige Reulenfanåmme und ifre $\mathfrak{W}_{\text {eid }}$. ftadelu (d)einen nidbts weiter als unvolfommene Anfoinge von fortfåken zu feyn, die zu einer Beit austrieben, Da Dab Hymenium fajon gebil= det ruar.

X. Der Rnótchenfanamm (Thelephora): Der Şut ift Sederartig, auf feiner IInterfeite mit ख̇arjden, zumeilen jedod) nur felten mit ver=

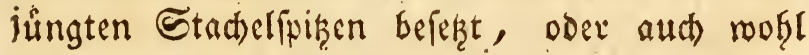
ganz einformig und glatt. Sa्due sarten Diefer (S)at= tung tragen ganz Deutlid) Das (Siepråge Der un: vollendeten Bildung. S(t) fterle mir die Gade un: gefåfir folgendermaffen vor. $\mathfrak{X}_{\text {enn }}$ ein Mycelium von einem Boletus, Merulius, Daedalea oder Hydnum rogen Mangel Deg Sodeng nidt genug 


\section{LXXXIV}

Nagrung exf̧ålt um feine Dodftindigkeit zu ex= Galten; fo fann ez zu jener Zcit wo eb die Ein=

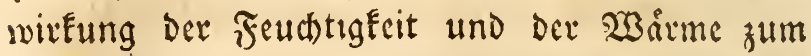
fructifiziren aus Dem Standorte Gervorlocfen, auch) Feine volfitindigen und mit allen Sorganen Der Regel verfefenen Encarpia hervorbringen.

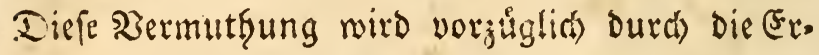
fafrung beftåttigt, oas Dergleidicn Thelephorae fid) nidst fo ordentlich von Jafre zu Jafir propa: giren, fondern nur fporedifh) eridssinen und wie=

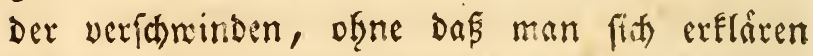
fonnte, aus meldem Sezirfe jie fergefommen, und wof̧in fie mieder genoandert fenn mogen. Bey Den maffren $\mathfrak{A}$ iten Der Sdwaimme fann man in allen Fillen ben 3 ug ifrer Propagation fo gut nie bey Den Pfionerogamen bemerken! Die 47 Arten Diefer EGrtgartiften Battung, welde mit

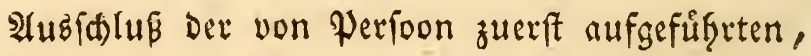
bey Bulliard, La Mark, Sowerby 4. D. gl. unter Peziza, Helvella und Auricularia gefudt wev: Den mulfen, theilt die Synopsis in folgende Saalb= gattungen ober Familien ab:

A. Craterella ( Şof̧lf(f) wamm). Der Şut ift vơlig rund, oberf̧alb auszefgofit, ober Irid)= terformig; Die Sdbeibe ftriegelidst - ftruppig. Die benden fiefere gefgorigen arten módten wofl ber:

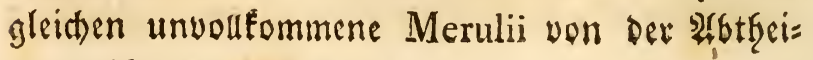
lung Chantarelli feyn! 
B. Stereum (Deffrleinfowamm). Der Şut if Galbirt und julegt wagrectst. Fith balte midh Durd) zaflreiche Seobachtungen fur ibergeugt,

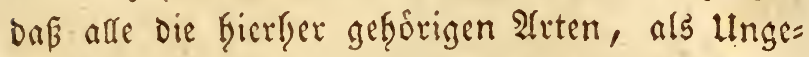
ftaltheiten, Dem Banderid)mamme (Boletus versicolor P.) mit feinen Ytbatten angeforen.

C. Corticium (Şautidimamm). Blok auggge= goffene, umgemendete, verfotedenartige $\widehat{\S}$ dhwomm= maffen, Die mit Sinotchen verfeften find. Die Arten diefer Familie find nact meiner Meynung nidstz weiter als werfiartete und vertecknete aus ifferm Soden bervorgemad fene Mycelien ofne Inflores= cenz. Sie find beftomefre Byffuartig je finfterer Der Sot war, in welderit fie entftanden find. Jm Biegentfeile find fie (jadertartig, und Durd) Die Sertrodfnung endich Sederartig. Die Papillen, wenn fie fid zeigen, find blope unvoufommene Infänge von einem Hymenium.

XI. Der Afffdfmamm: (Merisma). Der Sothamm ift aftig, Rederartig, zurammengedruckt, glatt, nur an Der Spike ift er offters f̧aarig. Die Merizmen find nach ifferer Subftanj Ieleptoo= ren, nad ifrere Geftalt bingegen Reulenfhno womme. Sie find bafer, midh freymútfig zut erflaren, mirffide (Slavatien von einer trofnen Subftans und fpezifild verfftiedener billoung, to mie fie aud) wirflid) fdon frůfee mit biefen vereiniget 


\section{LXXXVI}

maren. Siefeidht verfiart fich nuth Merisma ju Clavaria, mie Thelephora fu Boletus? Arten finden mir in perfoonz Synnpfe fieten.

XII. Der Reulenfowramm (Clavaria). (Fine balb einfadse, bald åftige Reule auf einem furzen nur felten Deutlichen S.trunfe, oder mit einem folden von ziemlider Diffe of ne Segeidinung ber Biange. Perfoon Gat 62 Irten, wovon mandse

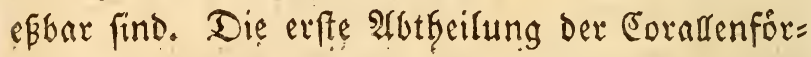
migen oder Aleftigen ( Solmbłioldz Ramaria) Gat Dep meiften $\mathfrak{A}$ nfprud auf bie Trennung und (Ex: bebung zu cincr eigenen Bjattung. Nur focinen

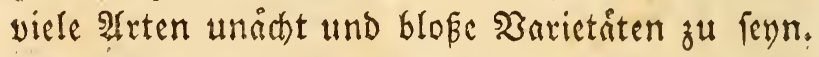

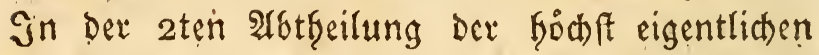
Seulenidnorimme find einige Irten Parainten von Juffeftenlaruen. Wras abe: won den bevoen Enol= Iidjten $\mathfrak{A}$ rten (Clav. granulata uno Phacorhịa) zu Gyalten fey, ift mix zur Zeit noch ein grobes

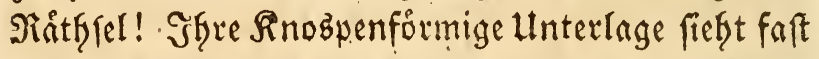
auz wie bie Zwiebel jener lebendig zeugenden Moożart (Dę Hypni annotini Web.) auz nel= d)er bie vormaflis von Roth und Seffimann foges nannte Trentepohlia erecta entipringet. Biefbt ez etroa nod) gar unter ber Reulenfdwoimmen nire: liche Sebendig gebaiterende d. i. Sinozpen alinerfende Biemaidter?

XIII. Der Erbtuingler (Geoglossum), Eine 
flcine, Furje, meiften's flact)gebrưcte, fleifhige fieuli mit cinem Flugelranss, Det aแb Dem Etrunts berauffeigt. Die fiefen arten dicket (jattung Denfe man fich als ctwas Flcine wenig åftige (d) lanfe Sieulenfdir amme mit flachen faft ebenen feft antiegenoen in oen Etrunt berntalaufenien Şiten; alío eine Aunáberung jul Mordbcl!

XIV. Der Epatelfdwamm (Spathularia). Reulcnithromm mit cinem breitgeoridcten, bâll= tigen Şute, weld)er ju 2 entgegengefersten Cei= ten fenfred)t nu dem Etrunfe bernbliuft. (5) giebt nut cine einjige art won biefer fefre aus = gejeidheten (battung.

XV. Dex spúthenfd)namm (Leotia). Ein Sipfiger Ectoramm, beffen rundlicher oder fie= gelformiger Şut fich ain Siande feffe einmaits umbieget und feinen Etrunf feft cinfdriefst. Diefs biattung welde aus 9 fefpe fritifaen uno werig befannten Arten beftebt, nafgert fich befondere De: non Geoglossum, obgleid) Die frute bier fres find. Áter es giebt ja audi unter Morchella Arten mit angemad) fenen und mit freven Shiten.

XVI. Dev Foltenfamamm (Helvella). Şat einen baukigen aufgeblafenen, fort unformliden, beyoerfeitz Gerabgebogenen Şut. Die Helv. acaulis ift wohl eine wafie Peziza. Die hbrigen? arten ftelfen eine Bintung bar, Die jwat mit 


\section{I.XXXVIII}

ber folgenden fefge nake verwande ift, aber dens nod) eine eigene zul fenn fheinet. Sie find mafre (cheinlid) alle espar.

XVII. Die Morthel (Morchella). Der ge= ftrectete Şut ift rund und voll Birůbden mit ges gitterten Raindern. Man Gat vormals mit gros Bem Unrectete diefe Srattung mit Phallus vereinigt. Selbft Bulliard uno Ventenat begiengen nod) bie=

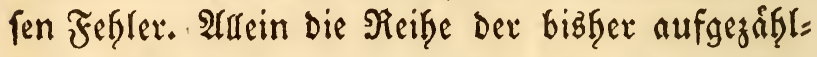
ten Biattungen wird zmeifelsofine den großen $\mathfrak{A} 6=$ ftand von Morchella und Phallus einem Jebree Den fưfifar madten; 8 Arten find in ber Sy= nople nufgenommen.

XVIII. Der Batertíthamm (Tremella). Eine Gingegoffene, Baallertartige, Rreisformigge= faltete Maffa. Die mefirften Gaben feinen eigen= thưulicten Şut. Man Gat bereitz Die Wafferge= waiche von biefer Bjattung aubigefbloffen und fie Den Pfycreen cinverleibt. In ber Folge modten woh! noch mefiecre von den 25 Arten diefer (jats tung verlofien gefen, und theils in andere úbers tragen, theils zu bloken monftrofen und ferilen

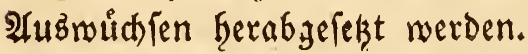

XIX. Der $\Xi_{d}$ úffelf $d$ wamm (Peziza). Det Galbfugeligte wertiefte, etwas angefthoollene, Sdufffelfórmige Fruct)tboden (ber Scut, ober bie Brutbef̧ålterbud) fe) fưfzet feine Brutbef̧âlter in 
LXXXIX

Der vertieften glatten Facibe. (Sektere find b̧ău= tig und Dem unbenaffneten $\mathscr{A}$ uge faum fidtbar, und fliegen gleich Crtabbeton Dawon, die meiften enthalten 8 Brutfornden). Diefe (battung bedarf nod) vieler Unterfud)ungen und bidftroafridein= lich ciner Bertheilung in mefrere. S(t) bin aud aflerbings der nafymliden Meynung, die fhon Şaberle a.a. D. ธ.73. geåuffert bat, daßj nåfm= fich ein grofer. Ifeil bavon gar niat unter bie Chwarmme, fondern unter die a!gen (in Die Familie Sarcocarpae in die Na(t)bariat)aft von Cyathus) fingefore, und das die (jattung Octospera dez̉ Sebnig nod) ferner beybefalten merben Diufte. Perioon Gat 151 arten, die er unter folgende Siubriquen eintbeilet:

A. Tremelloideae: (Bjaflertartige) find mefre oder meniger von der Subftanz des (3at=

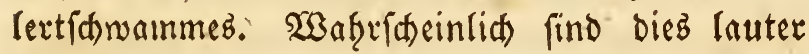
Sctopporen!

B. Helvelloideae: (Faltenføwammartige) find etwas gropker, fleifdig - Gåutig, jerbrechlich, auffen meflicht. Şief̧er gef̧ort bie Helvella acaulis!) $\mathfrak{A}$ (ud) unter biefen find viele Octosporae. C. Mreiftentfeils Eleine. Die Becter find yon auffen ftreifig, Gaarigt, ftriegelich, oder mollig uni feinfanrig. Seiefer gef̧ort unter andern auch die prådtige Peziza coccinea. 
$\mathrm{XC}$

D. Bians glatte, 23 arartig - fleirthige; mei= ftentfreils Fleine, Die wiederum in geftrunfte und in Strunflofe untergetfeifet werben. Die crffern find fefte artige Dingerdyen von ber Form einez Siboriumb oder Relerglafes aber zumeilen won suf farfer Zartbeit und fo Elein, bas man fie Eaum be= merfet.

E. Seterattige, trodne, glatte dier ftaubige groffentfeils of̧ne EtrunE. Diefe fobeinen an we= nigften in bicie siattung o $u$ waffen.

F. Stictis: (Napfpil?) eine eigene Battung, Die unter bie $\mathfrak{A l}_{\text {Igen }}$ ju ben Sarcocarpis in bie Rad,barjhaft von Naemaspora gefforet, und aud mirflich kereits bon mefreren glycologen nach bem

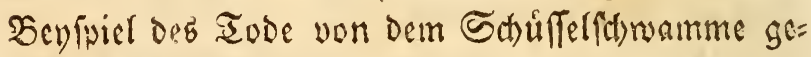
trent morden ift.

- G. Solenia (Trinfglabfhmamm). (sin faft Gäutiges geftrecktez, Schlauchformiges am Birunde

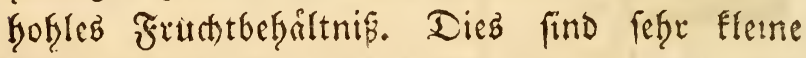
Schmainmchen, die viefeidt einzig gecignet fino in Diefer Bjattung und in Der Familie Der ádten Sdjmåmmic belaffen zu werden.

XX. Der $\widetilde{S}$ taud herfer (Ascobolus). Det Fruatitboden ift faft Papfformig, Shalbfugelrund, fleifdig. Die unverfenntaren Brutbefálter, ragen aus Der Brutbef̧ålterunterlage Gervor, werden zulekgt abgenorfen und fino meiftens mit 8 in 
einer Feudtigkeit if wimmenden Brutfornd)en

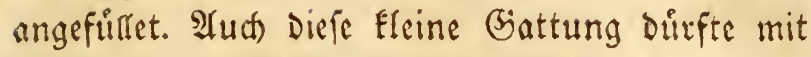
Sorred in Die Familic Der Sarcocarpium iber= tragen werben! Uebrigenz geben unz Diefe đatham =

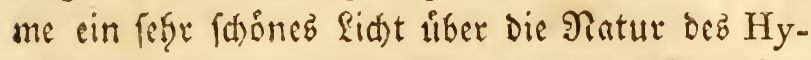
menii : Denn bey Diejen Pezizenartigen Schwân= men ift alld ber Frudtboden mit einer analogen Şaut befleidet, weldhe wie die placenta Der Dorstenia, von Den Piffifen Durdffochen wird und

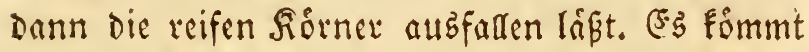
nun vorjughlid darauf an, of wir biefe herauzs getriebenen Saamenidhláude fúr wafire befgalter, coer nur für Zwirlingggeburten, wic bic 4 ङaa= men bey Der Didynamia gymnospermia Galten follen? (Fine Aufgabe fừ Die zufunftigen Beobact)= ter Der Edfwoimme!

XXI. Der Ropfichnamm (Helotium). Der Frudtboden (ein Şut) ift beftrunft, regelmaisig erf̧aben, fialbfugelig, unten und oben, wie bey Den Faltenidwaimmen, glatt, und fuffet feine brut an Der obern Fraethe, wie die Pejizen. Diefe

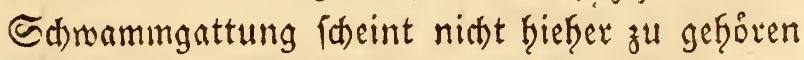

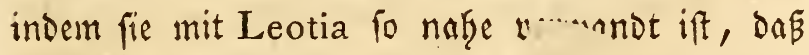
La Mark und Decandolle mefirere Arten Damit in eine Brattung vereinigt Gaber. Die úbrigen find vielleidt mahre Şelvellen! Ëz formmt f̧ier auf Die Fruhthaut bes Şutes an, ob fie auf Dex 


\section{XCII}

Skerfeite oder auf der untern gut Sónúrge wird! Perioon Gat nur 7 Irten.

XXII. Der Shimmerithramm (Stilbum). (Fin Sdimmelartiger beftrunfter Sdwamm, deffen volfes und rundes Rópfoten anfangs von ciner mâferidten und faft Biaflertartigen Eubftanz ift, ben feiner Reife aber undurd)jichtig und trúbe wird. Einige von Den 16 Arten biefer Biattung merfen das Ropjfthen ab, andere befalten eź, Doch vermeciet ez feftr bald auf bem Strunfe.

XXIll. Ier fióneridnamm (Aegerita). Strunfloie, Roornerformige, voufe Mef̧lattige Sdmámmaten. Diefe beyden resten Battungen Der Schnámme find den Sdjimmel und Soyfusar= tigen Biewaedsen fo nafe verwand, dak fie in ber Folge fefge maf̧rifheinlich bafgin uberefegt werden Durfter. Cie gefforen alfo zu den allereinfactften Bildungaformen ber Segetation, Die vielleint barinn beftegt, daß $\bar{\beta}$ cin abgetriebenez Giaffertar:

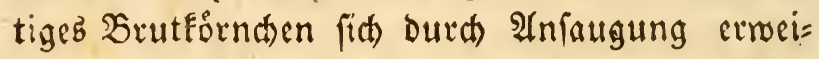
tert und endid) nieder ein neues aus feinem on nerften Gervortreibet.

Dies find nun bie fammtliden von Dr. Per= foon aufgeffellten Battungen der eigentliden Sdwnomme. Denn da mir die fungos Gymnocarpos für $\mathfrak{A l g e n ~ e x f l i r e n , ~ D e n e n ~ D e r ~ 6 t e n ~ D r o n u n g ~}$

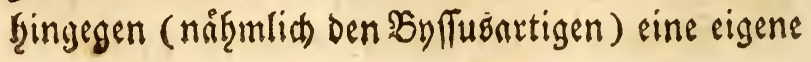




\section{XCIII}

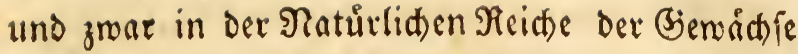
bie allerunterfte uno lekte Familie anmeifen; fo blieb unz für die Familie der Sthnomme in dic= fem ftrengen Sinne, wie fie hier genommen mers

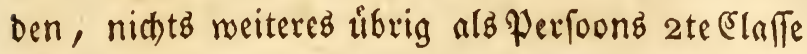
(Fungi gymnocarpi) und zmav aud) von biefer nux bie 4te uno 5te Dronung feiner Sabroimme, ne̊tzmlich feine Lytothecii und Hymenothecii.

$\mathfrak{u}_{\mathfrak{m}}$ aber in Den Bisift Diefer Methode beffer einzudringen, wollen mir jekt Diefes ganze biebieth Det eigentlid)en $\widetilde{S}$ dwaimme in einet tabetarifden Darftedung ůberfdauen.

momanomeneremenerem

\section{Familie der S屯wammartigen

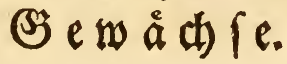
Erfte Familie. भืabre Sఝmámme.

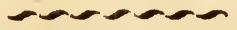

I. Aufiofungbgeburten: Ly tothecii.

1. Bitterfdramm: Clathrus.

2. (jidttidfwamm: Phallus.

II. Sdurirgeburten: Hymenothecii. A. Straf̧lenartige: Agaricoidei. 


\section{XCIV}

4. Blâtterfatwamm: Agaricus.

(3.) Sacfidmamm: Amanita.

a. Stiefelfđmamm: Lepiota.

b. Eoleyerfowamm: Cortinaria.

f. SOandelfdowamm: Pratella.

c. Tintenf(dramm: Coprinus.

d. Ragelifhnamm: Mycena.

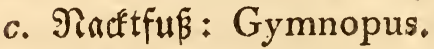

g. Srritling : Lactifluus.

$h$. Tribling: Russula.

$i$. Nabelifanamm: Omphalia.

k. S(t)mimmer : Pleuropus،

5. 2(Derforwamm: Merulius.

a. Pfefferting: Cantharellus.

B. Sôderartige: Boletoidei.

6. Sabyrintffidmamm: Daedalea.

5. b. Tfrånenfomamm: (Merulius) Seřo pula.

5. c. Şafenơfrtlein: (Merulius) Gomphus.

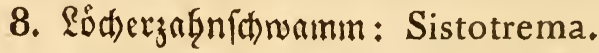

7. e. Fleifhlfhnamm: (Boletus) Fistulina.

7. Rócherfatwamm: Boletus.

a. Biffling: Suillus.

b. Stid\}[fomainm: Diatrepus.

c. Sturzichnamm: Poria.

7. d. Narbenifwamm: (Boletus) Poly- 
C. Nact thåutige: Gymnodermatoidei. 10. Finotctenftomanm: Thelephora. a. Soghliøwamm: Craterella. b. Defrleinichnamm: Stereum. 9. Stad)sifdwamm: Hydnum, (exceptis claväeformibus!)

10. c. Şautithramm: (Thelephora) Corticium.

D. Reulenfórmige: Clavaeformes. 9. b. Species $15 t a$ Jgelif)mamm. (Hydnum) Erinaceus.

9. d. Cornacnfotwamm (Hydnum) Hericium.

12. Reulenfdinamm: Clavaria.

11. Iffetwanm: Merisma

E. Faltentífte: Helveiloidei.

17. Mrorcticl: Morchella.

16. Faltenifinamm: Helvella.

14. Spatelfdwamm: Spathularia.

13. Frigưunglex: Geoglossum.

21. Rosfidinamm: Helotium.

15. Mưkedenfdtmamm: Leotia.

F. Banlertartige: Tremelloidei. 18. Ballertichnamm: Tremella.

G. Unåd)te und zweiferfafte Sdumammat=

19. ऽ๙uํfflfømamm: Peziza. tunger. 
a. Baulertartige: Tremelloideae,

b. Faltenidmammartige: Helvelloideae,

c. Sileine, fararidte: minores.

d. Silatte, $\mathfrak{X}$ Bactbartige: glabrae.

e. Sedérautige : Coriaceae.

f. গRapfpilze: Stictides.

19. g. Trinfglagfthrumm: Solenia.

20. S(t)lantherfer: Ascobolus.

22. Sd)immeridnamm: Stilbum.

23. Rórnerifhamm: Aegerita.

Die meiften Menfaten und felfift gefildete

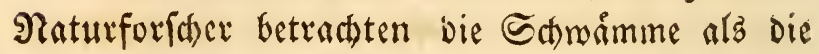
aderunvodfommenften uno veraidtlidften Pro= Dufte ber ganjen vaganifaen Sobjfung, und

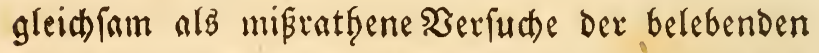
Natux, beren fie fich faft fiamen follte und bie fie alz ifgre verworfenften Stieffinder nur in bie abgelegenften und verborgenften WBinfel, in bie

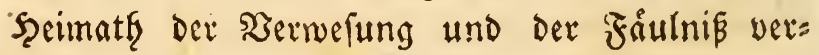
meifet. Die cha:afteriftifas Undanfbarfeit unferer Art geht fo meit, Daßj nir vorfeşlich oie $\mathfrak{A}$ ugen

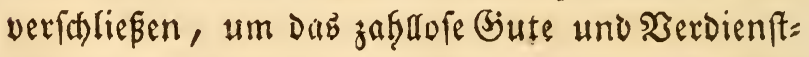
liche nidbt bamerken zu múffen, daz fie unz of̧ne

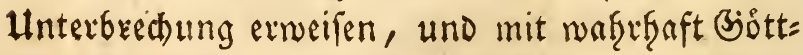

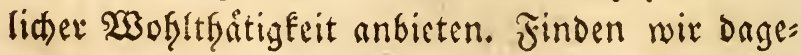

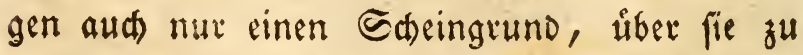
flagen und fie als unfere årgften IODfeinde zu 
veritbreven: 0 ! fo metteifern wir siner mit Dem andern, alle Bitterteit unfers Şergenz gegen fie aubjugieffen und fie alz Saftarde, als Samaroz=

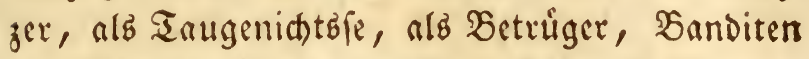
und Meud) ia nof̧l gat alz die gefäfrlichften Feinde ganzer menfhlid)er biemeinfpeiten zu laftern.

Wie ungeredt und grunolos diefe âdufäle und Angriffe auf Den Rubqm Der Sd) mámme find, fann man fich leid)t ủbergeugen, menn man nur umparthenrids genug ift, um folgende beyde M?o= tive ju (jemuth ju nef̧men, daß̧ nåbmlich exftens biz nuf biefe Zeit noch fefre roenig gefalefen ift, und nux fefre menige Menfiden fich die Muife ges nommen Gaben, die Ratur und (Figenid)afften Der Canromme zu ftudiren und ifie beftimmung in

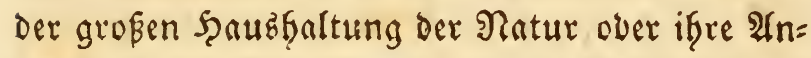
menoung zur 3efriebigung unferer ßeburfniß̧ zu erforfhen; Daß anderntheile an Den 习act theilen die wir von Diefen Befápofen exlitten f̧aben, nur unfere dicfe Unniffentgeit, unfere unverzeigliche Frågheit und Etumpfbeit $\sigma_{\text {chuld }}$ (ey, die uns

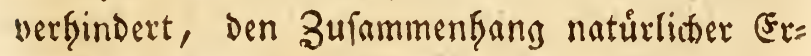
fheinungen mit ben 9 (bfichten bez biob)ften Bieiftes, Der affes nur nach ben weifeften und wohlthątigften Abfid)ten einleitet, zu errathen und zu begreifen! Und mas fie nid)t Eennet, was fie Eennen zu les: 


\section{XCVIII}

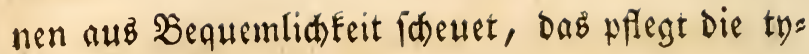
rannilabe Ulnwiffenteit jedergeit als unnůş, alz verdådtig, als gefáfgrlid) ju verrufen! Wrie follte man wohl yon unferm Zeitalter Auftlarung Der grósten uno intereffanteften Naturgefecimniß̧e er= warten, oa es nod) felbft unter ben 2 Xeifen biefer Periode fo irrfinnige Sopfiften gieft, melde bie Entfethung Der Solnåmme von ber Biąhrung Der

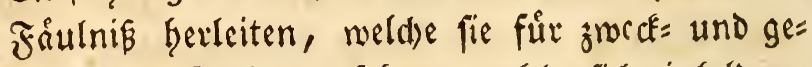
idhlechtlofe (jebilde anfefen, welde fith einbilden, ifre Bildung aus Der Mechanif, iffee Beftandtheile aนร Der Ehemie und ifere Mannigfaltigkeit aus Der Miatfematif ableiten uno exfláren zu fonnen! Woir Fennen fie alfo nodh gar nidt toder bod zum we= nigften nux fiod fit unvoufteindig biefe unfere fo ver

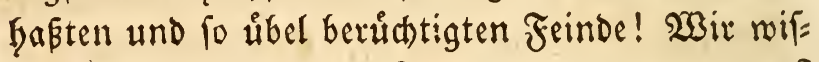
fen nidjt, wab fie find? nid)t, warum fie find? nicht, wie fie find? 23 แs wir aber von ifnen wif fen, ift nid)tż als ein purez

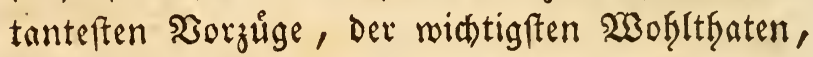
Der liebenenưrdigften Befáaligfeit - und von un= ferer Seite entgegen ber áufferft brutalen Ctupi= Ditât, Die mitten im Benuße ben Freund verflucht und verleffert, Der fid), offue barauf zu adten, nur Mrủbe giebt, feinen blinden Biegner mit Bjut=

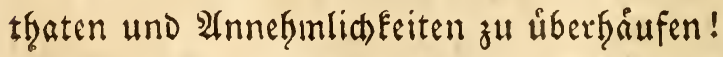

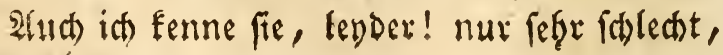


und, it) geftefee ese offentergig, id bin nidst wes niger einer won ben vielen llmwifienden, de fefre oft, gleich ungerat fenen Sindern, weld)e fict felbeft verderben, um nur bem woflmennenden Ermfte Des lef̧enden 2 aters iferen Muthwiden entaegen z"l femmen, fich an den edelfen Paturgaben Durdh Mişbraud) oder falife Zumutf̧ungen veriundiget faben. Dennod) wag idl es jekst, um einen Theil Des begangenen llntechts wieder gut zu machen, Die Efgere biefer Sdjopfung zu retten, uno aud Das $\mathfrak{X}$ enige, was mein Unucrmógen auf gen im Siande ift, mag viefleidht finteiden ifge mandsen $\mathfrak{D e}$ effere zu gewinmen, und fo manchen Diftatorifacn Y⿹anenoorfer mitten im Donner bes

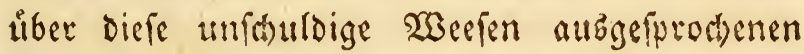
Inathems zum Sabweigen zu bringen.

Jich wial von Dem Biebraude beginnen, Den wir von Den Sdyaimmen macten. $\mathfrak{B}_{e n n}$ es auch nut menige $\mathfrak{A} r t e n$ find, Die wir mit Sicherffeit uno mit der forgfältigften $\mathfrak{A}$ uzmagl zum (Sinuße

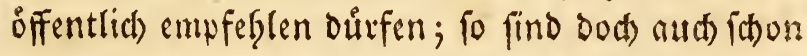
unter biefen mand)e fo ergiebig und fo fdrabbar, Dak wiv fie fiur nichts anderes als für cine Biabe Der Gimmlifasen Bilde unferer atemutter Ratur erfennen und annefimen Dưrien. Sis Eann mirb fier ber bündigften Rürge bedienen, Da id) bey Den ehen in defem Buthe felbft vorgetragenen Dlerfnut: 
C

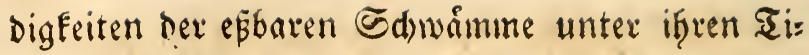
teln allez $\mathfrak{S}$ eefentliche gefagt zu fraben vermuthe. Şier muß ich) nur nod) die arnfidten vereinigen und meine Sefer barauf aufmerffam maden, Daß ç unter ben eßjbaren Sabroimmen folde giebt, bie (iic) Durd) Haut gout und Delicatesse ganz be=

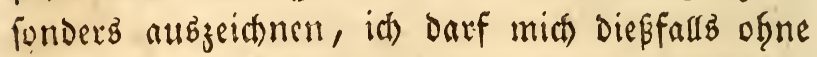
Zweifel vor aften Den ůbrigen auf bie Iruffel, nuf ben Raiferling, auf ben Egampignon, auf

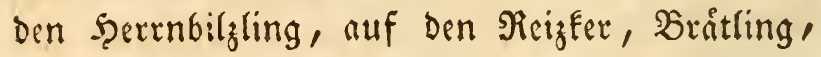
Rapling, Mrordel u. D. gl. berufen. Bey einigen andern $\mathfrak{A r t e n}$, wie bey Dem Rothlinge, Dem Şal=

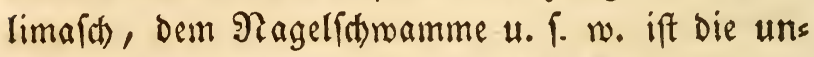
getheure Frengibigkeit fefre merfwurbig, momit Die Pantur fie unz in Den Sd)ook mirft. Die ês= baren @droimme fommen nidbt allein zu allen

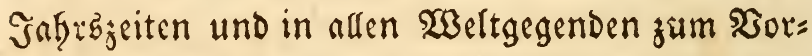
facin, felbft Bronland und Ramidatfa nid)t aus= senommen; fondern fie laffen fith aud in unfern

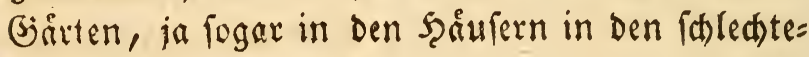
ften $2 B$ infeln, in Rellern und leeren Tonnen er= jeugen. Sie find Dafier millfommene Biafte, die

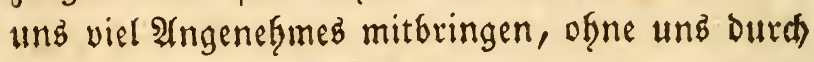
viele Foderungen von $\mathfrak{A}$ ufmerffamfeit zu geniren. Mit manden von biefen ङajwåmmen wirb großer Şandel getrieben und ganje Nationen leben faft sinzig von Den Sthmámmen. Man Eann fie zu 
afferfiand Recferfpeifen vermenden, man fann fie

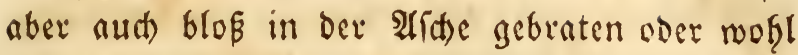
gar roh verfweifen. Man fann fie getrodfnet fư

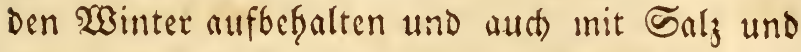
Esig voer mit Şaumol oder mit Zucfer condiren. Das nber bie fier nufgefúfrten Echrommarten

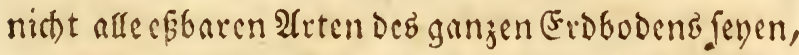
verfeft fich non felbft: Denn Die Poglen, die RuF fen, die Morgenlånder und die Amerifaner Gaben wieber ganz andere Arten, wie wir aub Den $23 \mathrm{Cl}=$ fen Der Seifenden j. B. aนż Pallaz, Şlirbaum, Midbaur u. D. gl. abnefimen Eonnen.

Die tieffe 9 remuth findet in ben Tagen bes Mangels nod 3 uflucht und Rettung in Dem Full = Goin der Natur, wenn fie in Den 2 saldoerts Бd)wamme auffuchet, und felbft im SBinter giebt es nod Pláke und unterirbifa) (jeirten, no fie mit můtterlicher $\mathfrak{B o r f i c h t i g f e i t ~ d i e f e ~ G o n d e r l i n g e ~}$ fingepflangt und fur ben fdumachtenden ungluck =

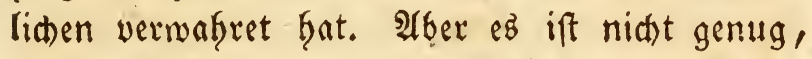
Daßs wir fier nur ber menigen $\widetilde{S}$ )nammarten $5 x=$ waignung madsen, die bereitz allgemrin fưr eßstar exfannt find. SBir fonnen vielmefre im Siegentbeile befaupten: (5) gebe nur febr menige gif= tige 5 droimme und a ud diefe waren c nur fur $\widetilde{\sigma}$ dwådlinge und $\sigma$ d) wel gex. 
cll

Da id) auf Den Punft Der $23 e r g i f t u n g$ in Diefer (sinleitung nod) einmafl zurieffommen werbe; fo will id midl) yor igt nidt langer pabey. auffialten.

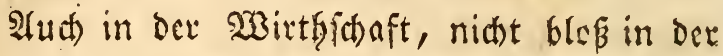
Siuthe, leiften uns bie Ect)wåmme erfeblithe Dienfte und fie wưrden uns genis noth viel meth= reve leiften, menn wir nur fleißiger barauf bedadit mairen, fie zu unterfuchen und zu beobactent. Die Zunderfhnormme, bie aller Drten fo ủber= (i) wenglich bånfig mad́len, fie liefern unz ein

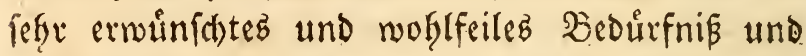
man fonnte fie nod) ưferbies zu alferley anderen Salbfithten verwenden. Bon allen záf̧en und Reder= artigen Sd)måmmen fann man Papiev mad)en. Itufferdem fonnten fidher viele Edtwåmme gleid Thicrifhen Tgheilen zut zabrifation von $\mathfrak{A}$ in $m o=$

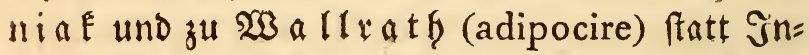
felt zu Seife uno lidetern benuk̨t merden! Sief̧e

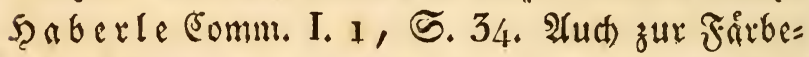
rey) find mande Satwamme anwendbar. Die Ed)wimmer, bie Tintenfanaimme und mefrere

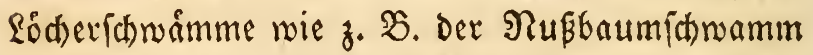
Boletus platyporus P. waren in diefer $\mathfrak{A}_{6}$ ficht

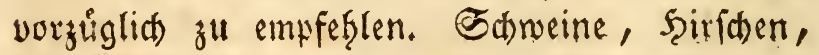

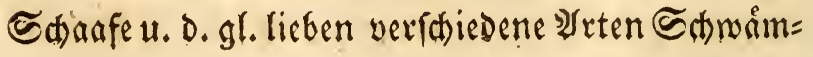
me und nafferen fith Davon, und in ber $\mathfrak{A}$ ragneys $=$ 
mittellefre verbienen bie Bunderfdynamme, Dex

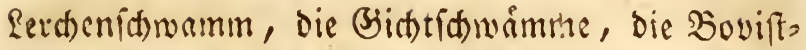
arten, Ser wohlriechende $23 e$ idenfhromm, (Daedalea suaveolens P.) Die Şirfabrunft (Sclero-

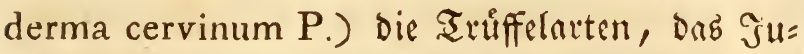

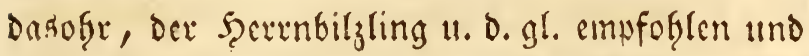

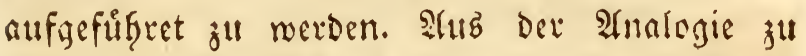

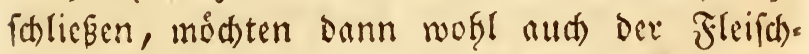

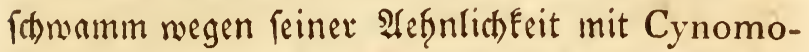
rium, ber Fliegenid)wamm, der Epeytrubling u. D. gl. midtige $\mathfrak{A}$ rzneyen abgeben fonnen und eine

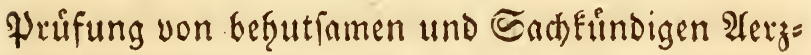
ten versienen.

Dod) wit molfen nun aud Der Beffinmung

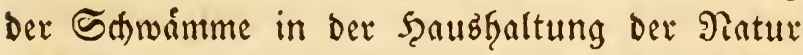
nachforfden! Frenlid mofl intereffirt biefe Unter: fuchung nur ben gebildeteren Theil meiner Refer: aber biefer ift's nud gerade felbft, an beffen $B e y=$

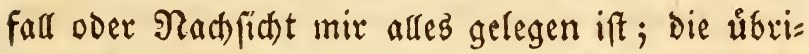
gen mogen mix bie fromme 9 (bfid)t zu buten hal=

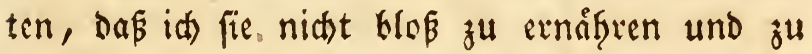
bereichern, fondern auth zu beffern und minder finnliden Meniden zu madten, dak ids ifren Beift aufaugeitern und auf cine ede des Menfthenran= ges mưrbige Weife zu unterf̧alten mưnfde. "Die "Menffen, fagt haberle* ) find oft wie bie

*) (Eomm. zu Berłud's Birberb. I, 1. p. 33. 


\section{CIV}

"Elcinen Sinder, fie wollen alles was iffnen bie "Natur Darbietet, nur immer ins Mlaul ftecfen.

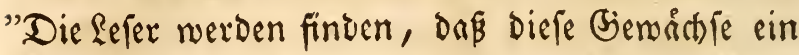
"Fefer ernftgaftes Spielzeug für die Mleniden wer= "Den fonnen, wenn fie die Paturtfátigkeit bey de= "ren Entftefiung verfolgen wollen." Dod felbft alb= Dann; menn feltnere shifíne die Şarmonie Der Sdopfung zu jerftogeen fiteinen, enoigt fie mit

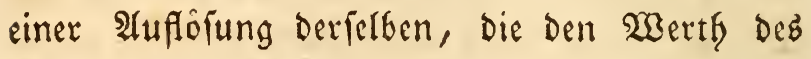
Saonen ůber fith felbft erfight und die felbft biefe vermeintlicten Diffonanzen in $\mathfrak{S O H}_{\text {olflang }}$ und b: jaubernde Anmuth vermandelt. Der Tfrainen= fidwamm, von weldem id oben geredet Gabe, foll unz bicfes durch sin Beyfpiel begreiflict) mas d)en! $23 e n n$ wir alles mit gebúfrender $\mathfrak{A}$ ufmert: famfeit in Siedanfen refapituliten was ich von den Eigenld)aften Diefes fo metfmürbigen Edmammes gemeldet habe; to ift won den vielen Infictiten,

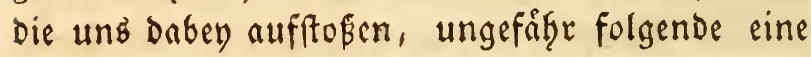
Der erften; Daß́, Da derfelte nun cinmafgl beftimmt ift, bie Gibaiude, won welden ex befith genom=

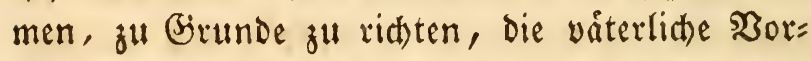
ficht Des Sdjopfers an bemfelben zugleich unver= fennbar fey, indem er bie Mienfichen, die fie be= wofinen, Durd) feinen faft unertrigliden Bieftanf bey Zeiten marnet, ja woffl mit Bienalt zwinget; fie efger zu verlaffen ald nod) iffr Einftur Dab 
Reben Derfelben bedrofet. Inderfeitz formen wir uns für ubergengt balten, das bie Plage dieles Satrammes nid)ts noiter als eine erefutive (5in: quartierung und eine woflverdicnte Strafe ley und dns fie mit zu den vielen God)ft weifen sinftal=

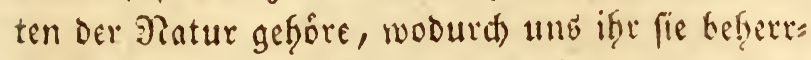
fdender Bienius zminget, die Echoprfung, aus ber wir unauffortid) unfere bedutrfniffe cottef̧nen,

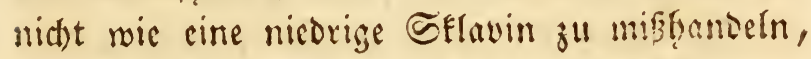

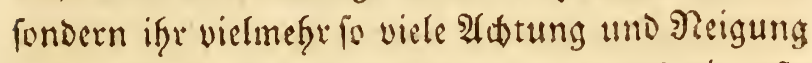
juzumenden, onf $\mathfrak{e}$ s einemieden von unz die erfte uno die intereffantefte angelegenfgeit fenn forlte, ifge Berfaffung, ifge 3mede uno ifre Drittel, su Diefen Zwecken zu gelangen, mit âfufwand allev unferer Breiftesfähigkeiten zu ftudien. raeitere Fortianritte in Dex Naturfunde, nur Diefe fino das Sedingnif und mir merden unfere Bebåude fo an:

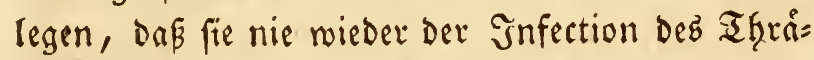
nenichnammes unterworfen find, und neder feine nod) Die Iflianen der Unglưcfliden merden bie (je: måder Der Eterblid)en beneken, wenn åd)ter (Sie= braud) Der Sernunft uno grundlide Renntnip Der Natux ifge Şandlungen leiten! Denn, wenn

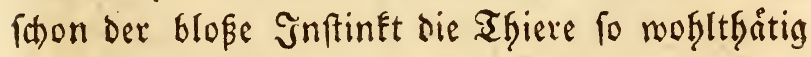
bewafiret, wie wir es aftågli(h) beobadten fónnen;

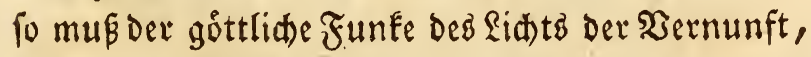
Der unfere $\mathfrak{B}$ eefenticit fo meit iffer die Shierwelt 


\section{CVI}

exfeft, unb nod) weit fidserer und meit mirffamer bemactstigen, aus dein Eabyrinth Dex Serfailtniffe

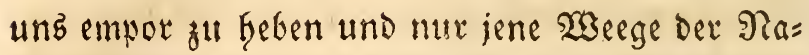
tur zu ergreifen, Deren Sitd)tung Das Zier unferer Beftimmung unfeflbar erreichet! Bulliard berect)= net, Daß Sdnoimme in einem beftimmten Zeit= raume ungefábre 600 Mal to viel 233 affer cinfaus gen als andere (jewaidse, bie in bem Eroboden 23urzel faffen. Ex foließt hieraub: ,que la $\mathrm{Na}$ ture emploie ces Champignons comme autant de régulateurs pour le maintien de l'équilibre, si nécessaire entre les fluides et les solides; il me semble voir un contre poids d'un effet prompt et sûr, lequel est toujours prêt à être mis en action, des que le cas le requiert." ${ }^{*}$ ) Heberfaupt verdienen die Sdynamme von diefer Seite, fo wie bie Myriaden von Jnfeften und Wourmern, als unfere geffeimen $2 B o f$ !thăter und als fefre thåtige beamte ber natúrlid)en polizey betradtet zu merden. 2Ales mas fault, allez, was mit feinen mekfitifhen 2 (uzbunftungen bie ?uft, Die mir unaufgorlid einatfmen und erneuern múf

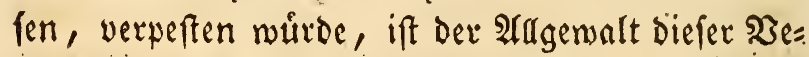
getrbilien unterworfen, die ç in furzer Zeit auf: zeffeen und umwandeln. Und menn aud welde von Dielen geldaftigen Polizeyfoldaten felbft wieder zum.

*) Bull. Hist. des Champ. I, p. 64 . 
(Senuß unbraudbar find und, wer weís aus nel dien Urfadsen, nicht braudbar feyn follen; fo bat die 9atux an ifnen bod alles getfran, was fie thun fonnte, sm fie uns fofihbar und mexterux: sig jul machen, da fie biefelben bald sureh bie Anmuth ifree (Sieftalt und Durch Die ausgefuchte=

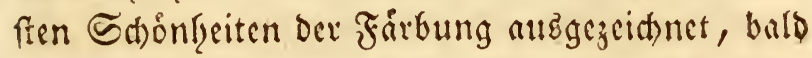

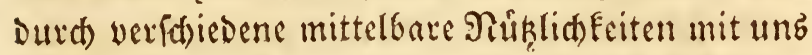
wicber augzufofnen verfuctst Gat, Da fie nafonlich entweder alb Arzneymittel, alz Fabrifationgftoffe

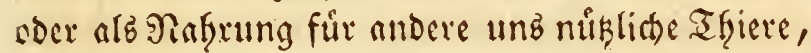

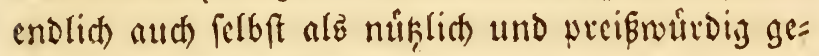
xulfint ju werden verdienen.

In Dex Igat, es ift ein xuffrender und Serz: erf̧ebender Anblicf, die faft an Eitelfeit grånzende Bemuffung dex Natur geroafir zu nefimen, womit fie fich ereifert, allez şấliche binneg ơ fanffen,

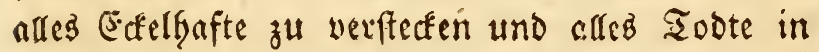
neues jugenolirbes Seben zu vernandeln. Wer die

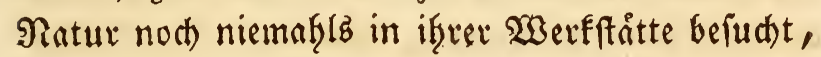
id) modnte licber fagen; bey ifrer Soilette uber= rafat bat: Dex gebe hinaus in Den $\mathfrak{W}_{\text {ald }}$ und be= trad)te die reizende (jiuppirung Der Erofanwamme, vornaf̧mlid) Die Der Bilglinge, oer Säublinge, ber Prâtlinge u. D. gl., Der fefge wie fie im aubgefaul= ten Pappelbaume ungef̧eure J)iaffen vom Boletus citrinus nufgetfiurmet, die eine Pradt baeftellen, 


\section{CVIII}

Der alle Slumen Indienz weichen; ber unterfudbe

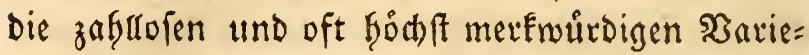
tåten Der Sainderfdwaimme und alle bie 23 under: geftalten yon Echwormmen die die (5id)e und der WBeidenbaum Gervorbringer. 23 er aber nut allein

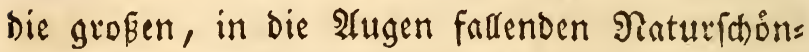
Geiten feiner $\mathfrak{A} u$ fmerffamfeit mưrsigen noute, Der wurbe fith einen großen, ja bey meitem ben gróz= ten Theil ifreer Ergóbungen entziefen. Daz \$ers gróßerungżglab entbeçt unz nid)t allein die (jies Geimnise Der Srganifation, fondern audi folde

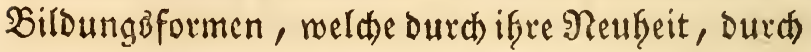
Zeidnung und Farben, Durd) Enntraft und $\mathfrak{A}$ na logie und fefar oft burd) geniffe unz ủberrafdende Erinnerungen und Ieffnlidfeiten mit ganz andern Dingen in ser Seele dez Beobadters wafres Ent= zůcten ermecten and ifm einen Beidmact ein= flópen, Der feinen Neigungen auf Die Zeit feinez

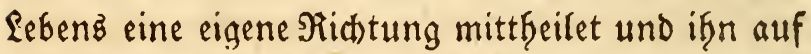
emig vor allen verfefirten, thoridten und Ratur= wibrigen begierden vermafiet, $2 B e r$ einmafgl mit Erfolg in Diefe Biefilde ber optifaen Schoufung eingedrungen, ber fennt itr Zukunft nur eine einzige feidenfant, bie allez úbernoindende liebe

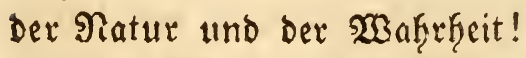

Sm Wald und Feld, auf Bergen und in Bruinden 
CIX

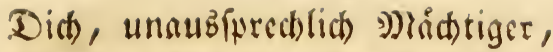

Unendlich) Buter, Seerrlicter,

Bott, aller 23 elten Biott! Did futhen und Did finden;

Selbft ba, noct) ba, dich finden, nie bu bift Und wie Du mirkeft, Du, Deß Raf̧me Geilig ift, Woffin Dem edflen Stolz den Blict zu riditen grauct,

W3ofin logar Der (jeiz nid)t (d)auet, WBeld)' eine Secligfeit!

D Ruft, Durd) Fein Bieniffenzkagen, Rein Ungeftumm im Şerzen und im Mlagen, アidat Durd) Trambjendentalititen und Feinen Qualm Der Jnterpreten Beftumpft, verbittert und entwengt!

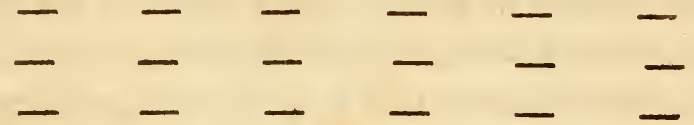

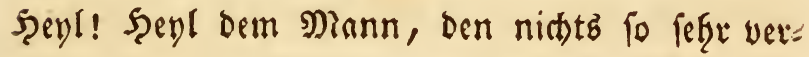
gnuigt,

Alb ůberall nach biott zu fragen,

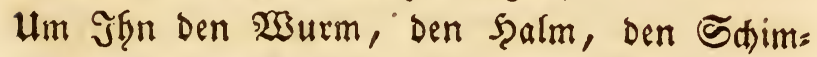
mel zu befragen!

Denn fonder antwort merden fie Fưrwafir ifgn nie entlaffen; aber wie,

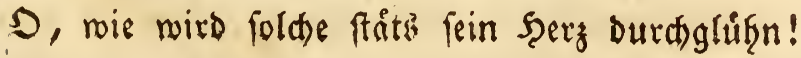
wie wird 


\section{CX}

Den milloen Forft, Den ex Durdirtet

Sin einen Tempel fdineal vermandelt ex erblicketr!

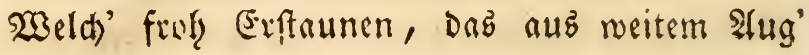

ifim quiat!

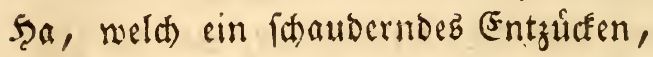

Das mit bes nafen Bott's biefúf̨l fein Scer' Dur(d)Dringt!

Tode.

Dech nicit allein Silloung und Farbe fteteen Der গratur bey Der Familie Der ๔a)noimme zu (je: botfe. Sfgnen ift aud Die lieblidsfte Parfumerie ber eilien und Rofen nid)t frembe. Der Duft bez moflriedanden Weibenidnammes fo wie dee bez Rautfiffnammes (Boletus fumosus) affmet Dem Savian (Illicium anisatum) nad) und farint ifn nod) an Feinfecit zu ubertueffen. Fs ift in Der That fefr frappant, fo elegante(sichoopfe in ber falechteften Sceimath faulender und ganz vermoridster 23 eiden= båme ju finven. So cntwidfelt fich aber audt) zu=

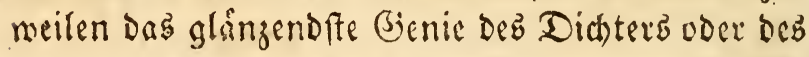
Fyelden in ber elendeften Dunfelfeit einer armfee= ligen Esrohgutte, obcr nofhl gar 5 Irepwen foct) in Der Dadfufube bez Ednneidera?

Dic Ceratophora Fribergensis deg Şum= bolot (Werfoonz Boletus odoratus verbreitet einen fefle angenefimen $\mathfrak{B e i l ( t ) e n g e r u t h}$, menn fie vet:

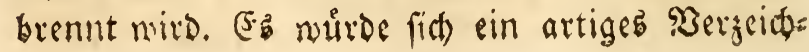




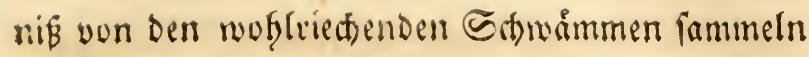
Iafien; aber id muß abbrect)en um nictst uber die

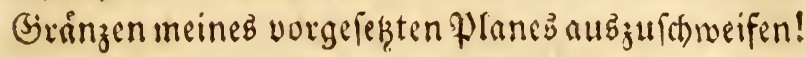

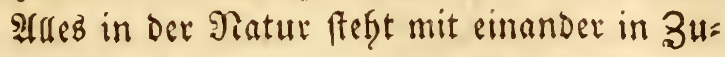
fammentyang, eines ift um des andern wiflen da. (jâbe ez z. B. Feine jnfeften; fo wurben fo viele

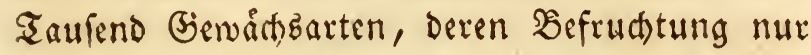
won ifrex Bevfưlfe nobångt, aubfterben, und viele unferer nüliaften Şausthiere wurben vor Şunger verfamadten. Wir Fennen indeffen diefe Nothmendigkeit aller Exiftenzen uno ifrer $\mathfrak{X}$ ex richtungen im Detnil noch fefre wenig: aber mir fennen genug Davon um uns zu ưbergeugen, Dnß aud) Die ưbrigen Arten, Deren Seftimmung in Der Şaubgaltung Der Natur wir nod) nid)t exforfat Gaben, eine affnliche, wenigftens eben to meife und eben to wohlthatige Beftimmung faben múk fen. Unter ber zafllofen Menge der mifrofozmi-

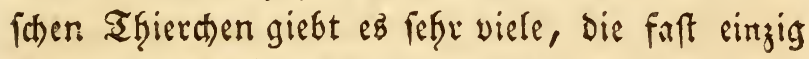
von Den Edyraimmen zu leben fateinen, in man murde fogar vor nicht gax langer Beit in Den Jre mafin gefůfrt, daß die Shroimme feloft Iffiere,

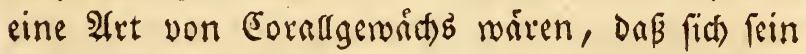
Saus Pflanzenformig baute, wie fo vicle andere Boopfyten Der Mecre. Diefer Grotfum nurde

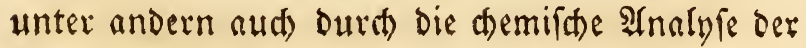
Sdhormme befrefiftigt, da man fand, dap fie auf 


\section{CXII}

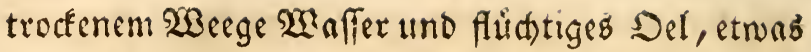
Rofilenfaures bias abor nur bidhit menig Rof = lenftoff geben, sie worguglictife Hrfache ifres fonellen 23 adththumes, on fich dieles immex im ungefefrten Sirfâltniffe gegen die Menge bes vorbandenen fioflenftoffez jeiget! Ialein die (Fi: fafirung muste uns auch bald micder eines beffern belefren, dof roffmlich alle diefe Sfierden nut (3iafte und Feine Seftendtheile der Echnarmme find; zumafil Die Arten biefer Parafnten ofterz in einem und bemielten Ectromme verfachicden uni zun Sheile auth fhon von unfern (Entomologen benannt find. alle diefe Shierd)en nun leben Şordenneife

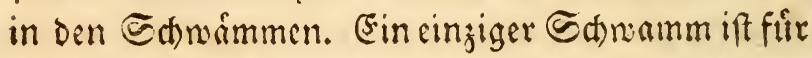
fie nicht sin Şaus - nein! eine großje volferidte Sauptitndt! fie nåbren fich Davon, fie berwotgnen diefelben, fie finden allerler Etoffe zu ifren ander: meitigen Beburfniffen in benfelben. Daßj aud) an= Dere Ifiere die $\widetilde{S}$ hroemme lieben und fie mit $b_{e}=$ gierde aufluctien, ift theils fiton gemeldet morden,

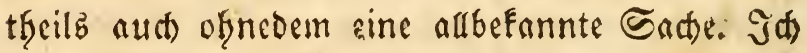
finde es demnach ůberflůfig, fierorts nod) mefir Davon ju erråfinen uno begnůge mid mit ber blopen Erinnerung an Diefe Beftimmung ber ธdi)nåmme.

Die meiften Eamammarten foreinen 2 fafro: Difiacalifate Rrafte zu befiken und Durch Den (jies 
nußs mitjuţeilen. Non oen Irriffeln, wom (bid)t, formanme, von hem fliegenidnamme baten wil Erfafrungen, die diclez auffallend beweifen. Da

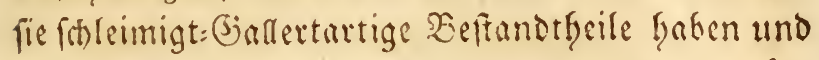
cine Suantitait mackten foulen entfalten; fo lifit

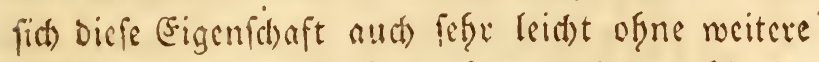
Exflarung begreifen. Fie mognen onfer noff vies Ien Antbeil an Der Bewolferang fomohl Der thieri= faben alb Der Penfd)enwelt neffmen und die weni= gen letfralen Fifle, die fie veranlast baben, find Demnach auf Der andern Ecite wicber reid) lid) Dur(t) Die geftarkte Bcugung $\xi_{\text {Faft }}$ ucrgutet!

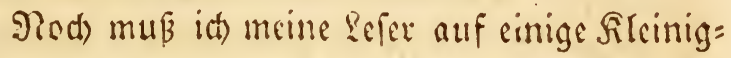
feiten aufmerffan mact)en, Die muhl einft alb Die Duefle midtiger und groker folgen angeref̧en netden Durften. Durch ocri (sictud) Der Ect)rumme merden viele ₹ficle, oft aus grofen Entfernungen, berbey gelockt. $\sigma$ ch a f fer erjâflt von Dem (jid)t= fhnomme, Daf fein fataler Bieftanf eine Mienge Jnfecten ferbey geloct, und baß er Demfelben den

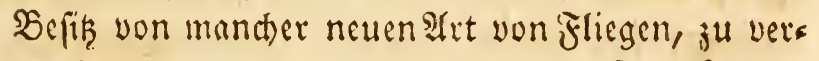
Danfen fabe. Wix fefen nun in Den Sabrommen ein Stimulans, Das die SGiermelt in Senegung

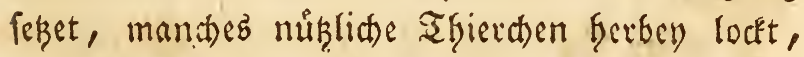
Das viefleicht diefer (biegend faflt, oder andere von ifrem Heberflube befieyet!

Die parafytifben Sobromme (Denn aud) Sุ 


\section{CXIV}

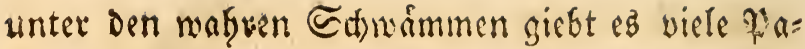
rafoten!) fonnten in Zufunft mofl aud ein leffe= reidies Rennzeithen liber bie Natur und Eigen=

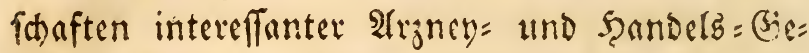
sorate abgeben. Denn menn wit aud nitflich unter Dem beißen Eroghirtel cinen banum antreffen forten, auf reldem bie nêfimliden Sd)mammat: ten wohnen, die mir in Furopa o. (5. auf unfern (Eid)en antreffen; fo wưrde ez feinem Bmeifel unter= liegen, daß er acelimatifirt werden fonne und daß ex úterbieg aud) afle bie Eigenifaften unferer (Fi= den befike. (Fin Meffr und ein 2 Beniger, Das bie Sathfundigen mofil zu unteridseiden miffen, ift bey sifen Dingen zu beobadten!

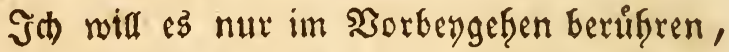

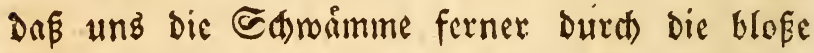
Miannigfaltigkeit ifrer oft fefre frappanten Formen

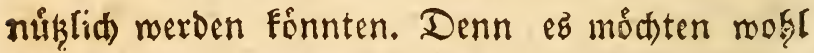
mand) Darunter gefdaffen feyn, in dem (jeifte irgend sines Miatfematifers oder cines Maftsiniften auf

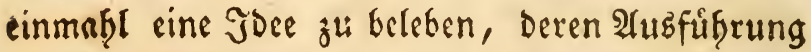

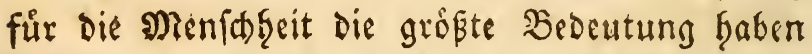
fonnte!

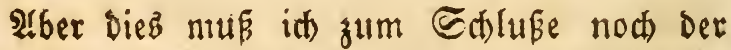
Heberlegung meiner Sejer Dreis geben, daßj ez die Shwaimme fino, Denen wir alle Seurbarung der Exde mittelbar und urfprůnglid ju verdanfen hăas 
ben. Rein Siena be findet Rafrung in ber roben yon aufgelópten Deiganifarionen noch ungefdona

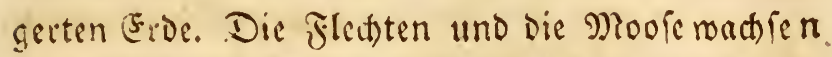
groai aud auf Eaflem Bieitein: allein geroofntich

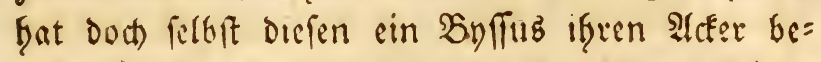
fellet. Gelbft da, wo roix nidjtz fefgen, mógen

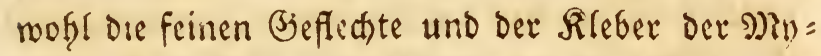
celien fineinoringen, und fo natb einer Reife von

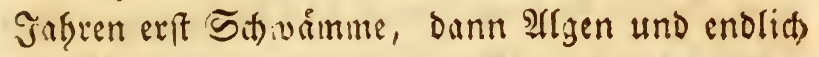
aud) andere (jicruathfe empor fommen.

sBenn wir nun biefes alles wobl befergigen und alle Norurtheile aus unferer Seele verban: nen; fo fefgen wir ung gegnungen, unfere úber=

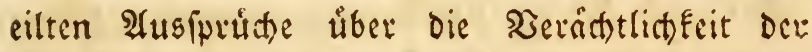
Echnåmme zutude ju nef̧men uno fie vielmefir fur die belten fleinoden im Diadem Det Blumen= gottinn zu exEennen!

গRun liegr es mix noch ob, alles mas mes: fentlich urd exprobt ift ûber Die (sicnubbrifeit uni ûber die Sifte der $\lesssim$ chnamme ju verjammeln.

Die frifhen, unjerlegten Beftandtheile Der

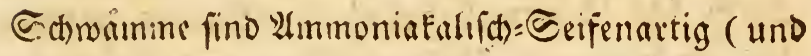
mante fatt lluinss) mef̧r cour roeniger ofglubt,

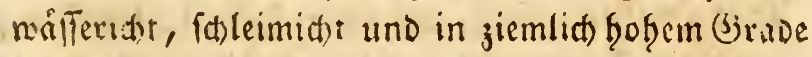
gallarta:tig. wab dem allen fönnen wir faliefen, baß fie Jur giafgrung geeignet und wenn gleich 


\section{CXVI}

weniget als die Fleifhlfeifen, Doch aud, mefre als alle die $\mathfrak{u}_{b}$ rigen 2 egetabilien nafibhaft find.

Da aber die Eubftang ber Ednurmme in

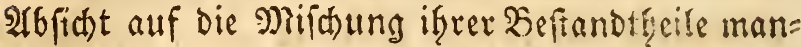
derley Serfacoenfeiten unternorfen iff; fo folget

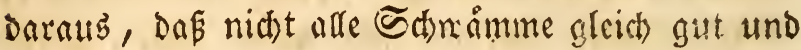
einige derfelben nur Dartum ungeniestar find, weil ifre esoerartige oder Giolzerme Diaffe fich meder weid) fod)en, no(b) neniger aber im Minger, ju einem

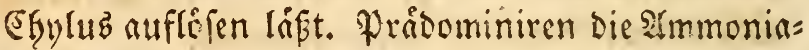
Falifhen Subftanjen; fo Fann ber Ed)mamm zmar meid) und angenefin, aber bennoch regen afzu=

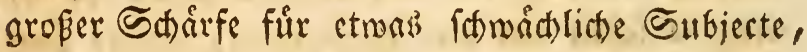
oder auth für die ftarfften, in allugrofer Menge eingenommen, (d) áblich feyn. Eigentlich narfotif(be Rråfte fobeint Fein Edwramm zu befiken. Die Wirfungen Der Sabrómme, die Den Enmptomen Der narfotifaen (Bifte affnlid), find, Durften siels mefir inmpatfetifa won ber Affection dez Magenz und ber Biedarme, alb von mas anderem ferruf ren. Frifhe und reine Eabramme f́chaden alfo nut Durd) Das Ueberma der angegebenen Seftandtheile, und in diefem

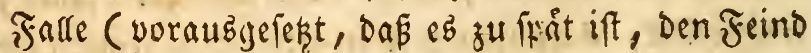
Durch ein Siredsmittel aub Dem Seibe zu (d)affen!) fann freylich mofl, wie $\mathfrak{F} x \mathfrak{a f}$ unz belefret, Dab pure falte Sorunnenwaffer, fowoḩl innerlic 
CXVII

genommen, als aukerlich im Ulmíd)lage angemen= Det, die erwuinfaten Diente leiften.

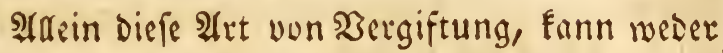
fefre gemófnlid, nod) von grof̧er Scocutung lenn! Sn Den meiften Fålen gefdieft fie, nach meiner voftligen Heberzeugung, Gloß Durch Den Misbraud und die Unflugheit Derjenigen, die fie aufnefgmen und jubereiten. Sabroimme, bic noch im unrei= fen, jungfráliden Zuftande gepflúft morden, geficn meder in fructification, nod in fralung uber. Sgaben fie aber cimmaft die Periode ifger vofligen Entmidklung erreidet; fo bringen fie Saa

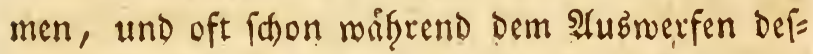
felben fangen fie aud an zu verberben. Ed)on der

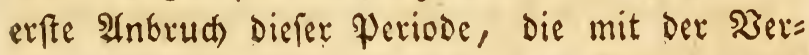
ftreuung Des Pollen oder mit Der $\mathfrak{A}$ usouftung Der in ifm enthaltenen Saamenfeuchtigkeit beginnet, lodet eine Mienge Ungeziefers fierbey, die fich refge fanell Darinn einniftelt und vermefret.

Weld)er von diefen Dreven der Sauptumftand

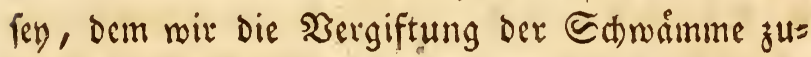
(d)eiben múffen, weif id nid)t beftimmt anju=

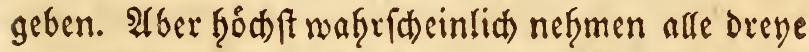
ifren Antheil Datan: Denn faule Sioxper, zumal wenn fie fegr mafferig find, exfennet die ganze Welt fúr ungelund. Das mit den Edwarmmen verfaluste Ungeziefer, mag entroeder animalifore 
Cxill

(Sifte entroideln, ober man ingerint fie nod les bend und fie bringen ourch iff Etrauben uno Sincipen in Den Eingemeiden einen Reis frervor,

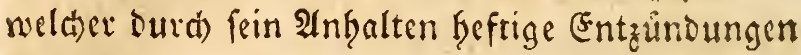

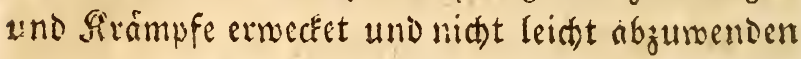
ift, indem riefe Ihierden fo flein find, bas fie

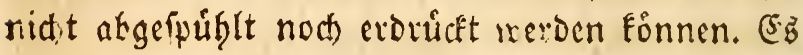
giebt aber mefreere Ifhiergattungen, von nelden mir miffen, Daß fie ein to zoffes Seben baben, da

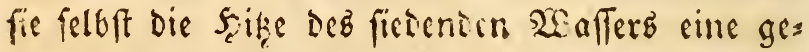
zaume Zeit finnurd) extragen fonnen. Folglid)

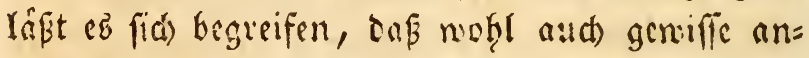
Dere ?aten nod sine Jeit lang in unferen (singe= weiben fortleben migen, wenn fie mit anoeren Zingen werishlucft morten, ober dap felbft ein ges Yindes Siod)en fie nidit notfiwendiger 23 eife tobten mufte.

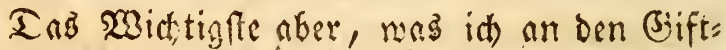
fdmámmen fúv Den angुreifenden Theil fyalte, find - Die Eaamen ser Ecthámme. Norfidtige

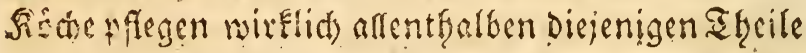
abzulofen wno finmeg ju werfen, die mir bereits aus scm 2ierlatife Dicfex Einlcitung ale dab $\mathrm{Hy}$ -

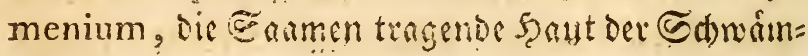
me fennen giternt Eqaken. Sirlein mandthal mag Felbft im Fleifine nod) ein Theil von diefen Eaa= men doer waht nud bom Polten ftecten bleiten! 
Dicje Eanmentrut mag ferter bey mandsen

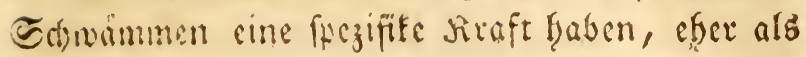
nndere in unferen Eingerveloen ju feimen, D. h. ju jerkloben uno ein anfangenteg Mycelium ju cotfalten! Da nun aber jedco organifathe 23 es fen, fo lange es lebt, in feinem anbern leben Fann, ofne mit ifm im Siamp zu fenn; fo ers

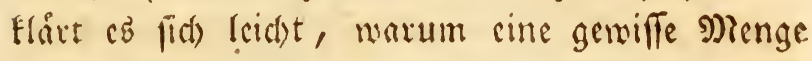
folcher Ed)wanmesime in unfercm Miagen grope Unoronungen amidsten muffe, on fie fich darinir feftzufesen, anjufaugen uno eingunifteln bemúber,

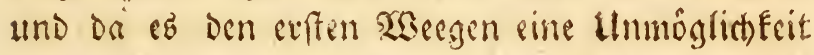
ift, biefelben, fo lange fie leben und entgegens wirfen, fortjufthaffen uno in uniere Subftans

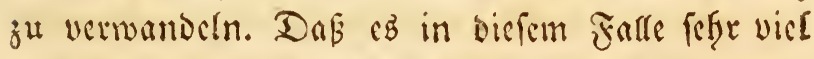
allf bie Integrität uno Die Siraft unferer Digeftion anfomme, begreift fitit von ferfften, und bic fucs

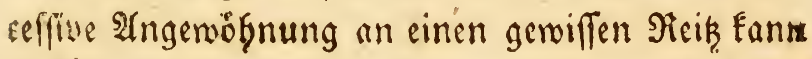
audi) beniefen, una mir ifn enolid) ertragen lers nen, ofne ifn weiter laftig zu finden. Die Empfin= Dung coftumpfenre Mittel (Paregorica) (d)eimen Dafier am meiften angegcigt zu fern, bey cince Sd)wanmuergiftung Diefer lebten $\mathfrak{A}_{\mathfrak{t} t}^{*}$ ).

Jil) babe aud) Des Polfen ermáfinet; uno Diefer mag manthmal mit Den Reits vermeţeren fiets

*) Jeboct nur in fo lange, als nod feine (Entgimbung rops

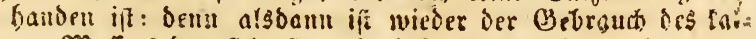
ten Maffers das fitherfie wno einfadfie Specificum? 


\section{CXX}

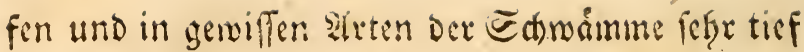

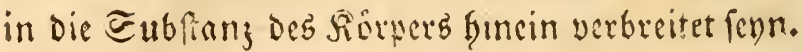
Dies vermuthe ith instefondere von jencn âten

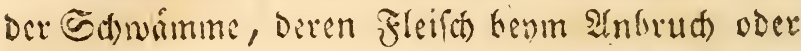
Durdict)nitt fraflenformig anlauft unv hruin oder livid mi:d, welthe Farbe wir iedegmath aud) an den fiey liegenden fifigelden ber beyden Eerual= organe in Dem Sgymenium bes Ectrommes ex= bitcten.

Wirl man fid alfo vor Sergiftungen Durd)

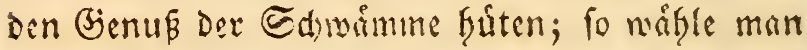
nor ber Sand feine anderen, als weldse fyier als ç̄oar und gefabrloz find aufgeftellet worden; und nill man ia aud andere verfud)en; fo refime man nux junge unentwickelte Sremplare, trenne $\mathfrak{Q} a$ mellen, Jiofgrden, Stad)ln und nab Dergleid)en mefy. Formen Des Şymeniums find, mit aller $\mathfrak{B o r}=$ fidt Davon ab, zetichneide ubrigens den Ed)wamm in Eleine Epalten, wafhe dicfe rein unv zu wic=

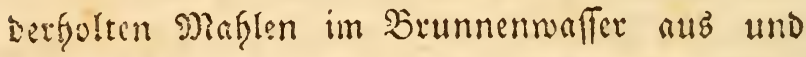

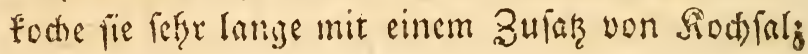
und 3nicbeln.

(5) giebt folledterbingż feine allgemeinen कo

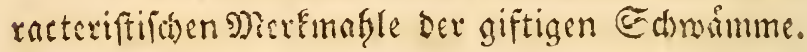
Doch find Dicjenigen, Die teym snbrudie fanelf Die Frotbe ifres Fleifás verandern und b!au oder

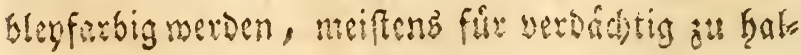


CXXI

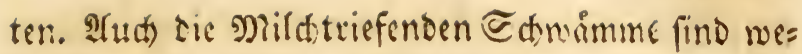
gen ifger Echarfe im Illgemeinen fitt betenflid) j"l halten.

Sisia man Edyamme trufen, ob fie chbar oder giftig kenen? To verdienen folgende Mietfoben als vorguglich empfoblen zu werben.

a) Man lege einen Eilberloffel und eine ge= fibalte zrviebel in Die Bruffe und laffe fie eine ge raume Zeit mit Den Ed,wammen ofyne anderen Zufas Focten. Sauft Der Soffel an oder nitd

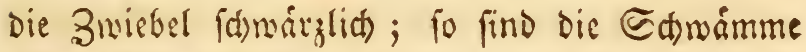
fur giftig zu gralten.

b) J)?an verfofte ein Etüfd)en Dawon gans wh und befalte es lange im Miunde. Zeigt fich

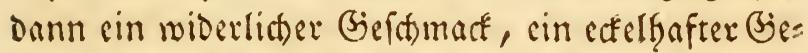
rud) oder sine Edhirfe, fo ift Die sirt, yon Der eg genommen ift, ebenfalls zu verneifen.

c) Mlan beobadte bie MletEmafle einmafil

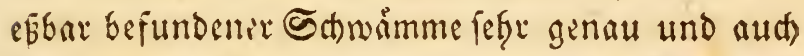
eine geringe 2 (bweidung in Der Farbe, made fie uns vetråct)tig!

d) Son Ectsmámmen, die man nid)t fef̧r wohl fennet, wage man es nicht, gleid die exfter Brafle eine grobere Dunntitít zu geniesen.

e) Dian fareibe nicht alif die Gauld be: Edrurmme mas etwa ein verdorbener Magen, cine úble Iiepofition ofer eine fefre wisernatir. 


\section{CXXIT}

Iide Sermifaung beterogerier Subftanzen zuneg? gebradt) bat.

Dnâ aber an oen ifblen Sirtungen ber Edroimme grósten Sheils unfere verterbte Satur und fraftlofe sijerdaunng nádit ter feflerbaft en

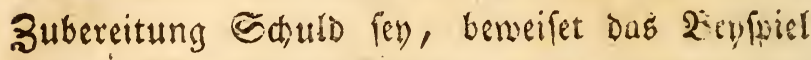

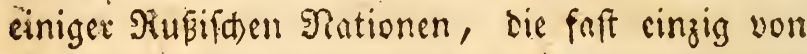
Sdmámmen leben und mit unter sud vicle bon Denjenigen vergefren, die rwir fur giftig halten, wie j. B. Die Silzlinge.

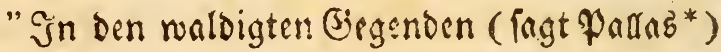

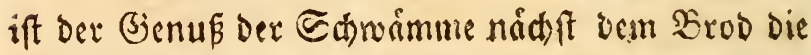
gemóhnliafte und faft einjige Faftenfpeife bes ax= men Sanovolkes, auf Den swinter roerden cinige Arten getrofnet; andere eingeinlzen, nufberoafret. Ueberf̧aupt geniept man in Mußland (den fliegen= fdroanum und einige fleine magern Pilze, auth die ftinfenden Driftidnoinme aug̉genommen) faft alle andere arten, aud), wenn fie faon murm= ftichig und Dem Untergange nafie find, und Dow f̧oret man nidst, Daßs diefe (Sieniád) fe, fo mie fie bex Lanomann fice ju genieken pflegt, neijmlids blok mit Ealj, eder mit Seflele gefotien, poer

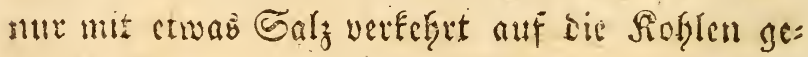

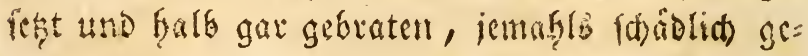

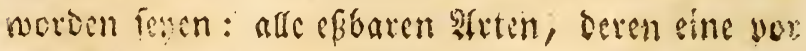

*; E. צuU. Meifn I Ib!. E. 43. 
Der andern brufiger ju entftefien \&flegen, meis

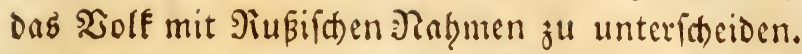
(5) find Darunter aud fold)e, weld the man in ans Dern Sendern als fhablich verwirft." u. f. w.

23ie viel die Berwofntgeit von Jugeno auf vermige, den Mlenichen felbft an Den Bienus der

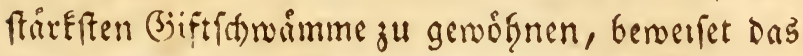
Beyfpiel Der Santíabalen und Der Roraten, dic Den Fliegeniatroamm effen, um fich zu beraufaren und in cine gerviffe 23 uth zu geratfert, wenn fie fu Feloe jiefen odex jemanden umbringen wollen. Ja die Begierde nach Diefem Benuj geft bey den Fioracen to neit; "que lorsqu'un homme en est yvre pour en avoir manger, ils ne lui permettent pas de pisserpar terre, mais ils luidonnent un vaisseau, et boivent son urine, s'imaginent, qu'elle produit le même effet, que le Champignon: la dose est de trois ou quatre, mais lorsqu'ils veulent s?enyvrer, ils en mangent jusqu’à dix $\stackrel{\prime *}{*}$ ).

* ) Krascheninnikow Histoire de Kantschatka etc. etc. trad. du Russien eic. Eie madien auch eine Irt von berauiten:

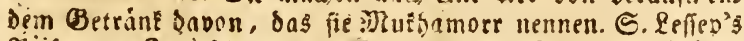

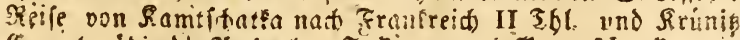

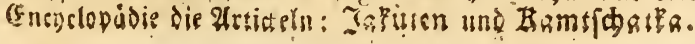





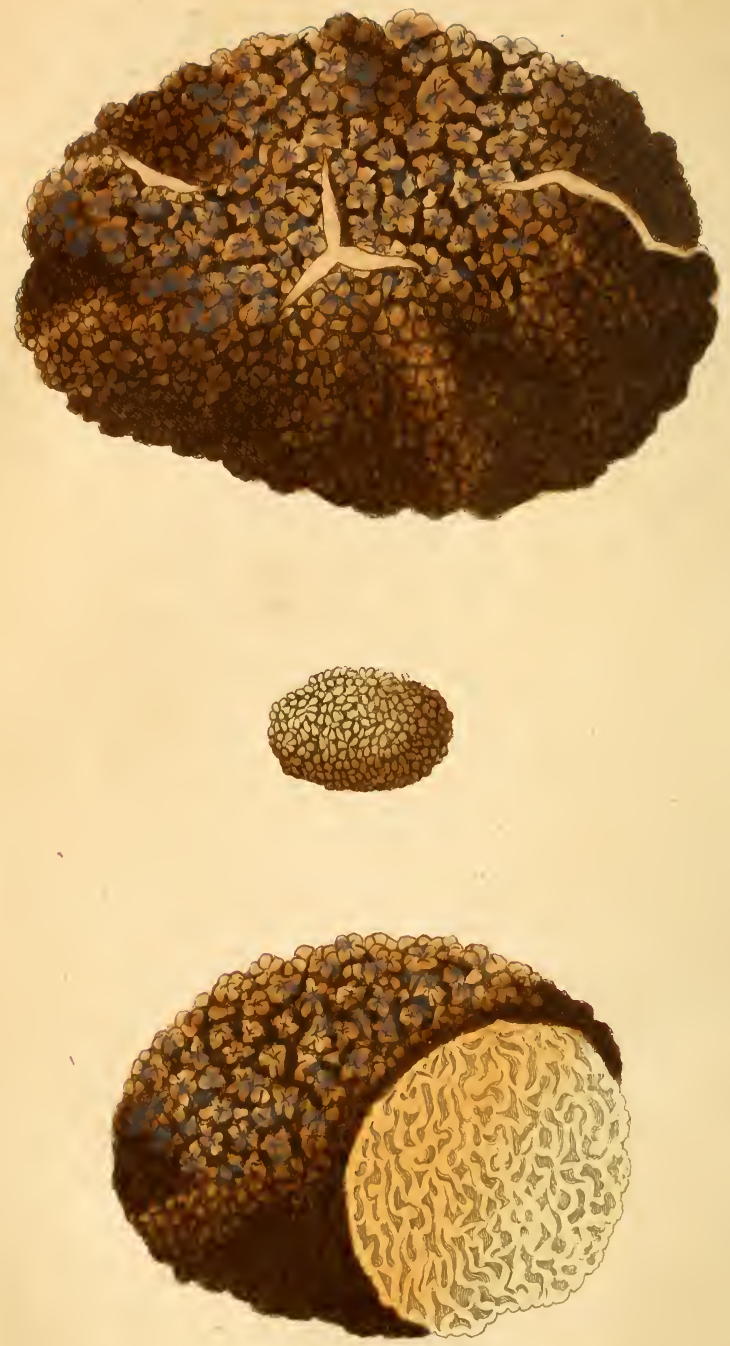

Tuber ritramenn. D. Vibth Die sichnaraye Trieffel. 


\section{Die Trúffel (Tuber cibarium Pers.)}

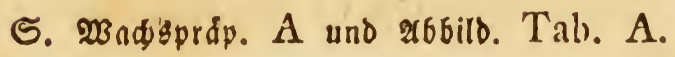

\section{(5)}

ie Trüffeln, von meldten fier bie Rebe iff, werden forlt aud die fitimarezen Trüffeln, Tars

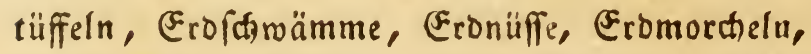
im Franjör. Truffes, Jtal. Tartnfi, Engl. esculent Puff-hall ober 'Trt:ffies, Span. Criadillas (Eeffifet), Dortug. Tortuthos, Şoll. Tartnffels, ooer auth Aardbuilen, Dän. Tröfo Ier, Søimed. Haräpple, Doln. Tartufule, Ruß̧. la Tartuffle, Ungar. Szarvas gomba oder Szarvaska genannr.

Ungeathtet fie nidtit ju ber eigentlidfen $F a$ milie ber Sthwämme gehören, weil fie ihre Forte pflanjungéf̈rner (oie Ṡtirammfeime, Sporu-

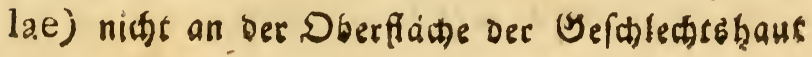


(Hymenium) Giervortreiben, fonbern fie inner, lich in einer $2 \mathrm{rt}$ von fruththülle berbergen: fo mijfen fie Dennow in einem $\mathfrak{B}$ erfe abgeţandelt werben, beffen şeffimmung Jopularität, deffen Eegentano Derienige 'sheil der Speilematerialien ift, welthen man im gemeinen leben mit bem Naksmen Der Satrömme ju bejeithen gewohnt ift, uno ben falt alle Sdriftifeller (bie allerneues ften allein ausgenommen) Denfrlben benjujäh̨len phegen.

Die Trüffeln wurben vormahls für sine ein. jige 2lrt von Rugelfowamm gehalten, unb bem zu Folge aud im sinneeiften Eerualinftem und vielen andern Berken unter ber Rener mung Lycoplerdon tuber aufgeführet. 2llsin mit vollem Pechte hat man in ben neuern Beiten bie Trüfel zu einer cigenen Battung erhob cn, und verfachiebes ne Irten berfelben nađ bem Berthältnifi iḅrer Bes fralt uno Farbe unteridjieden. So hat man bann jest 1) Dir Trüffel (Tuber cibarium), 2) Die meifie Trüffel (Tuber album), 3) bie graue Truffel (Tuber griseum), 4) Die Biefamtrüf。 fel (Tuber moschatum), 5) vie Sommertrüf, fel (Tuber aestivum), mit meldher lestern auch Die grünlidhe Trüffel Des 2llbertini und Sameis nit (Tuber virens) in eine einzige Art vereinis get zu renn ftheinet. Poch gibt é hier uno da Benennungen von Trüffelli, weldte einige andere 
bisher now unbeftimmte Irten anjuseigen fheis nen. Insbefonderk aber labe in voreinigen Jabs

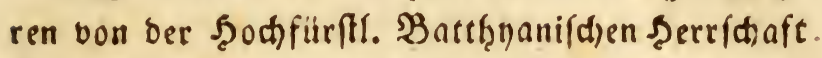
Enjereborf eine ämtlicte $\mathfrak{B}_{\text {enntwortung bes allo }}$ bcrt angeftellten $\mathfrak{W a l b}_{\text {, und }}$ Revieridgers und Walderaminators, Sęrrn Carl Meifters, auf 24 von mir entworfene die Naturgef(tict)te ber Truiffel betreffende Gragen erfialten, morunter bie I te 2lutwort eine neue $\mathfrak{l l}_{\mathrm{rt}}$ zu betreffen ftheinet, über beren näbere 2 fufflärung iđ mid biefter ver. geblich bemühet babe, genauere গadtricten ju

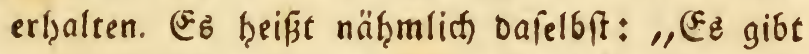
rothe (Triiffeln), welthe bie fleinften, meise, welche grüfiser, und fatwarje, relche bie gröften und (d) masthafteften find; bie rothen uno wels:n rachfen am bäufigften im Leimiकten (Lehmigen), bagegen die forwarzen nur im ithrarzen Bioden gefunden werden" :SBaE hier unter der rotheu Srüffel verftanden merbe, if mir gänzliat) untes Fannt; es müfite denn nur eine $21 b a r t$ ber foges nannten Sommeriruffel bun Den Förftern fo gea aannt werten.

Die Truffel ift ein unordentlich geformter, methrentheilo ziemlich enförmiger, sumellen aber auch ganj Eugelruniver Rnollen, an welctien man berfóiedene Einorüde und כertiefungen bemerkt, Die theils von dem Inneinanderlienen mehrerer Stücfe derielben, theils von dem Druck ber na* 
he baben befindidien Eteine, WBurgeln uno an, berer harten R̈̈rper hergutiąren fdjzinen. 2Lüer Diefen grüfern Ulnebenbeiten ift auch bie ganze Oberfiä()e berfelben mit geobängten niebergebricks ten und ganz frumpfáantigen Dyramiben, wie mit einer mineralificten Drufenrinde umgeben. Diefe find aber felpr unregelmiafig, 3, 4, 5 und mefrreitig, balo mit flacten, balo mit einges oritcten Geiten, und ithre Brößze varirt im Durd), meffir von I biz gu 3 \{imien, befonders nadj dem 2liter uno oer Reife ber Trüfel. Эiodh gifte ez jus weilen Silfe ober Epalten in ber Miinde ber Trüf, fel, wo bann bie meifliche Subftang hervurbricht, fo mie öfters 3. $\mathfrak{B}$. an ber 2tnanas die Edfale zerplatget, uno bas fotmacthafte Fleifth bervor.

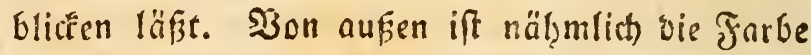

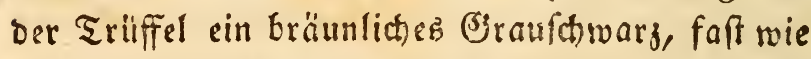
bey Dent Feberfarje Deer wie bey Derjenigen Zre

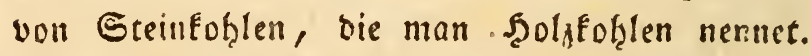
3on innen iff fie formuşigneí, mit zarten roth. bräunlichen 2loern marmorarrig burch zogen, unb freft baher im Durdfotnitt einer SRuǵtatmuß ziemliक ähnlidh. Im jüngern Buffanie find bende jarben, fowobl die äufere ale die innere, bels ler, und ourch bie $\mathfrak{B}$ :rweefung affimiliten fie fith entich wieder ber erde, in meldter fie entfanten.

Da bie Truffel nur unter ber Erde soäcthf, fo fotsint es nidbt nöthig zu fenn, fie mit alloern 


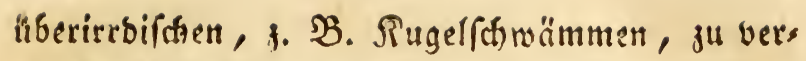

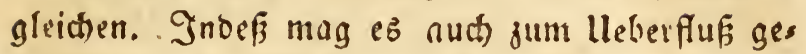
fagt Fenn, daß̧ die Truffil keine. Spur von einer

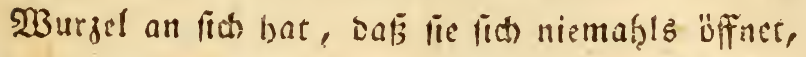
und dafi ifre Subfrant; fich niemable in Staub, Bren oder $\mathfrak{B}$ affer aufföjet. Ifm leid)teften fünnte fie allenfalle, jeooch ofme Bsefabr, mit ben iíris gen Trüffelarten oder mit der şirfthbrunft (Scleroderma carvinum) verwechfelt werben. Man barf jeboch nur bie rauţe brufenartige DGerflädse im Sebädteniffe behalten, fo ift cas bey allen übris gen $\mathfrak{B e r g e f f e n h e i t e n ~ u n m o ̈ g l i d h , ~ i n ~ e i n e n ~ f o l d i e n ~}$ כerffö zu gerathen.

Der B̈erud ber friffien Triliffel, weldher an

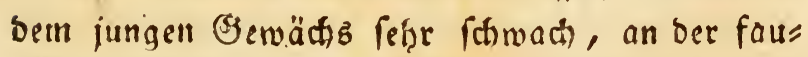
renden IJflanse bingegen abfheulich ift, fällt wätro

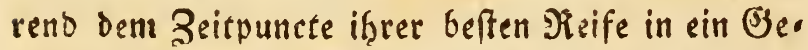

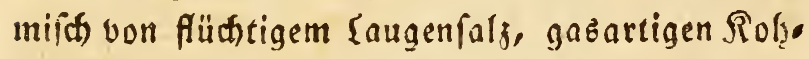
Senftoff unt ben Dämpfen von fodiendem Fleifáe. Der Bsefdmack ift methlicht, etrab reifenartig, und idh finte noch überbie in bemfelben eine bors

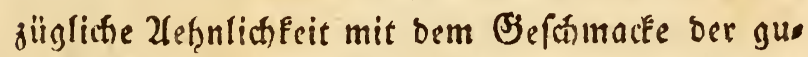
ten Raftanien, jeoot) gleidfan mit einer 20 ürrze von $R a l m u s$ und mit ciner fofmaden Spur bes

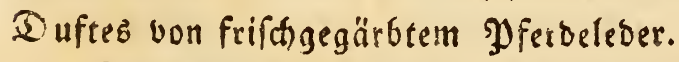

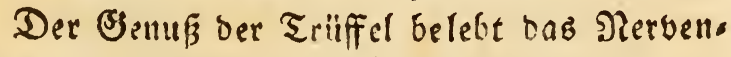
finftem ber Sayonopbagen mit einer wolliffigen RegGarfeit, gleidmie fo manche andere Jy fans 
zenprobucte aus ber Framilie ber Drdfibeen, ber Scitamineen, \{fifiaceen u. Dgl. Der Srunto bie,

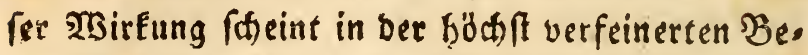
(d)affenteit ber nätrenden Subftanz zu beruhen, welche unveränbert von ben fymphatifich Eefä. fen in bas Seblüt abgefeftrt wiro, und bant ges rabe nur fo siel fu wentg homogenifirt ift, um in herienigen Drganen, weld)e aus ben leseten Enoigungen ber Blutgefäße ben Erraf̧ ber verlors nen $\mathfrak{B e f t a n t h t h e i l e ~ e r h a l t e n , ~ e i n e ~ u n g e w o ̈ h n t i d s e ~}$ Senfation zu erwedéln, obne fie jur gänzlicten 2B:rfftiefung bes Duthjuges zu reizen. Biels leithe hat auth wirtlid), wie Saller uno einige Intrere zu vetmuthen follitell, bie Subftanz bee

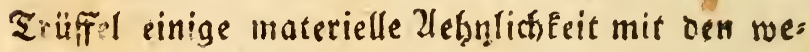
fentliden Subfranzen des thierifiten Rörpers ober mit osm Zeugungsffoffe. Bum menigften läft bet Se uh, unb felbit ber Befchmact berielten, eine foldte Bermuthung nicht ganj of he Rectitfertigung. IIno wenn es alfo iff, fo wiro es uin fo viel bes

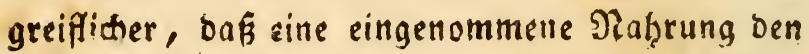
Seftelectitberieb ermedtet, weil fie in bem Rörper bażjenine Reizmittel lierbreitet, weldhes unter geo wiffen Umftänden von ber Natur felbft harvorges bradt werben follte, aber vislleidt) wegen irgent einer Stockung in Den Befäß̨̧n ober reegen Er. nüuung ber 2Rervenfraft mangelt. Die Trüfel ift bemnach ein 2tphrobifiacum, unto jwar von vors 
süglider Sottung, eine wohlthätige Sabe beg şimmeíz für biejentigen, bie iifrer beoürfen. Dee fo melar follet unto mögen fie aber audt) biejenio gen meioen, welde onju Eeine Berarraffung laa ben, bamit fie nistit in Gefolpe gerathen, mit einem innerlichen Feinde zu fämpfen, uno eine glamine zu vertilgen, bie fo leitht ein heillofes linglücf anriditen Fanu, genöthiget merben, ba es weit leidfter iff, ben eriten aló ben leşten Bes fahren zu entweid)an. Dogleid bie Trüfel, wie aus bem Bisherigen erf̧ellet, cin unfdäblidhes

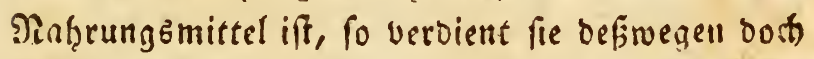
nimt unbedingt empfohten zu imerben. SMan hat Erfaţrungen, fagt Souttuin, baß seute burd) ben unmä̈̈igen Bebraud) den Tod bavon gegef. fen haben. Man Eam fid nun frenlid wohl aud

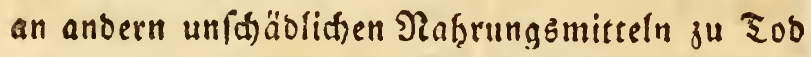
effen unb zu Tob trinfen. 2lber bod an mandien fribher! alle reigenden Subftangen find in oiefer

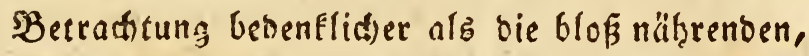

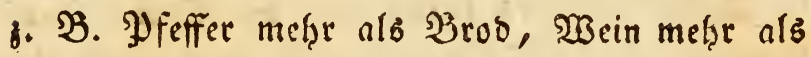
2Baffer, Salz melęr alz Şonig u. f. w. Zllein auch nodh aus anbern lirfacten ift ber Benuf́ ber Triiffeln, to wie ber Benuß aller 2lrtell von Sd) wämmen, minber empfeţlunģ̧⿻їrbig. Wenn näḩmlich bie Trüfel anti nur im geringften Brao be überreif geworben, fo pfegen firt zabilofe Sd) maroḩer auz bem seere ber Junecten baben 
8

cingufinden', bie ifere Laknrinthe mit haftlger Oes fdätigfeit ourctroliblen, und cie, iroem fie filtr fith nur \{ohn uno Befriebigung ärnoten, sugleict) ben ifgnen von ber SRutur angewiefenen $Y_{0}$ ftell beftellen, ullo eille ithres michtigfen Boprufeges fthăfte erfüllen, inoem fie bas Jaulende hinneg. fhaffen und die neue Ergeugung beföbern. Denn ba bie Trüffel von fich felbif fin nitht öfinct, und nod) ubrroief unter ser (Erbe begraben liegt, fo würbe fie fid weber fortpflanzen nody vermebren fönnen, welln nicht folde bienffertige Sebülfen beftellt wären, bie ihre fieim: befrenen und von eimer Stelle jur andern bringen müffen. 2lber eben biefe Infecten find es aud), Deren Begen, wart entweber bie Subfranj ser Sarwämme vers giftet, ober bie vielleicft, was mir Das Wubr,

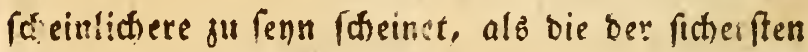
Beugen einer bercitb burch Alter uno Faulung gif tig gemorenen Neefenheit erfärt wetoin muf.

In fith Setrachtet, und mit Seintanfésuris al. Ier 2orurtbeile bes \{urus uro der Mobe, ver. bient eह bie Truffil bey weitem nicht, bon ben

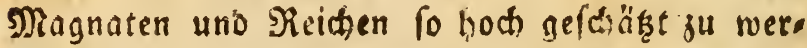
ben, als fie es von ben älteften ̧eiten zu fen)n pllegte. Man tönnte, ben einer gewiffen Buberei, tung, an taufens andern Dingen bielleicht einen eben fo guten, bielleidt einen nod) beffern Ses

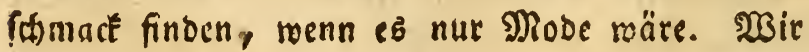


Pehen mitleibig auf unfere fraftwollen Stammuä. ter, wern wir im Tacitub lefen, Daß fie fid von

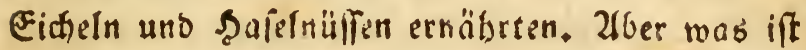
roohl für cin anderer llaterfthico unter den betus tigen und ben bamabligen \{ebetimitteln, ale cins zig in ber gröfern Mannigfaltigkeit uno in oer

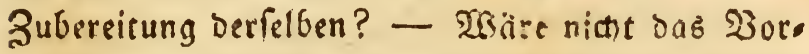
urtheil allmähtig, die beutiche Esctiel nütdo baib das arabiface bift der Eoffeeftande, und ber bas terländifte Salmus ben meftindifien Gngmer ver. brängen. Die Trüffel fet immerbin noblried)ento, ebel, föftlich, nabrínaft, gefuno, heilfam; -

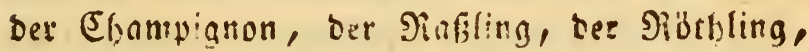
ber Bsolobrätling, ber siagelichromm, bie Sjior. Wel und der f̧errnpilzling fitid eह nitht un fo gax viel neniger. - Dod) cE fen ferne von mir, daßן ich) biefer \{ieblingsipcife einiger Tüufielfreunde itgo ren Barth befämpfen wollte! Es ift in fo mans den \{ämberil, wo nicht bie 马äger one Monopol

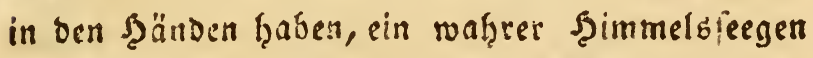
für bie armen \{anoleute, baj fie fith mit bem ohnehin müb̧amen Zuffuthen biefer Ifferfotwäm. me cinizes Selo veroienen mögen!

Man Eauft bie Trilffeln nat) bem (Sewidte. Die Jyreife aber find fehtr ungleid, nath 23eridice. Dentheit ber Jabrazeit und bes beffern oder fallectern Sebeibenz. 3u Marete pflegen fie bey unz nictst gebratit zu werden, fondern bie Epecrentgänder 
Ibjen fo!che in größzern Quantitäten benen Şerrs fhaften, Jägern oocr \{anbleuten ab, uno ob fie gleich bie meiften aus Ulngarn, Mäthren uno Sten. ermare, ja Feloft aus Defiterreid erbalten, fo milffen foldhe bemungeadtet $\mathfrak{b}$ enm Berfaufe fïr italiänifhe und franjöfifa)e gelten. EFhemable, als

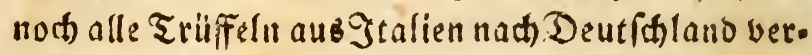
finrieban wurben, Eoflete bas \$fund 10 Thlr., und niát felten nod) mehre. Seitoem find fie meit über bie Sälfte im Dreife gefallen. Doch ift es fhwer, ben bem gegenwärtig to walloelbaren Merthe aller

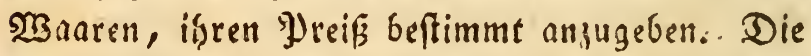
SRaylänbifhen in Delyl eingelegten Eofteten vor eis nigin Safien bas 2 fund zwen ober bren Thaler. Die 3inaigriers in Jyaris verfaufen aud Truffes marinées. 2tber feleft in Franfreich ift ifte

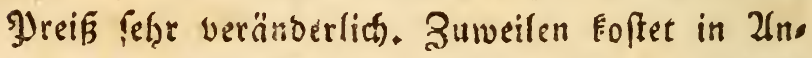
goumvis das Jjfund nur 15 bis 20 Sols (näţnulid) iun S. I779), ur.b wenige Tage here nath wohl Ico Soí, menn nähmlid farfer Froft und S. finee einfïllt. Für reiche Tafeln mirb auff) bort wohl cine Trifffsl, bie von vorzüglider Buite ift, und ein YJfunb miegt, für eitren \{ouiss b'or gekaufe. Tadt samburg fommen diefe Sd) wämme in Föffern ober Riften aug Bourbeauk und Marfille. (S. Bitkmanns Borb. fur Maarentunbe, 2. T(2. S. 72 ). Man pflegte übrigens die neiften aublänbifwen Trüffeln; forotgl bie 
eingemadien als dic marinirten, ill Deutiffland aus 2fir, 2tvignon, Bourbeaur, Eerte uno Diz. ja ju verfitreiben.

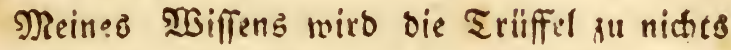
weiter als jur Speife verwendet, und juar nue für bie ausgefucteften Tafeln. 3war pfiggen fie hier und ba in ङegenben, wo fie bäufig gefunden merben, bon ben Sammlern felbit genoffen zu werben, indem fie folde blofi in ber 2fiche braten uno bann die Şaut abfकälen ober abfhabe:t, Man bringt fie nath 2rt ber Raftanien gefraten uno gefältt uno in eine Servicte eingervidelt auf bie Tafel, mo fie bann, weil fie marm erf̧altell wers ben, ben fo keliebten Duft nicht allein im 3im. mer, fonbern wohl im ganjen Saufe verberiten. Mrnnate pliegen fie nuch mit Butter ju röften, nachoem fie fie borber in sarte Sd)eibdjen zerfanit. ten. Die alten $\Re$ ömer pflegten fie mit Dehl, Pref. fer unb WBein zujubereiten. Zuf ben Tafeln oer 3ornefinen erfacinen fie ḩeut ju Tage felten als eigentlid)es (Serict), fonbern meiftens nur alb

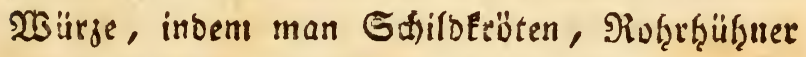
uno verfoticone Fifhe Damit jubereiter, uno its Berbinoung mit Ëbftidien Brüben, Eitronenfatt und allerlen Şewäzen auftiffier. IIIn vollenos die ganje 2Bürze Des Trüffelouftes in Den Serithten in erfialten, pflegen die Röbre die abgelofiten 
Stralen mitfutodien, und erft vo: bem 2lnridten Der Spcife foldte wieder herausjzunthmen.

Die alten Brriccten uno Siömer Eannten uno ver,

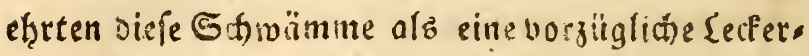

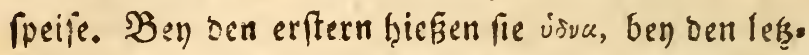
tern tubera terrae. Sie geben, vorgüglich झुlis niub, if̧re Rennzeidjen, itgren Stanoort, ihre Fars

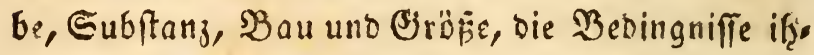

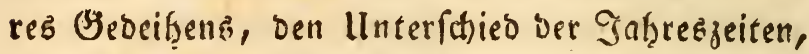
ifre Dauer und Berwelung, enditit auth fogac

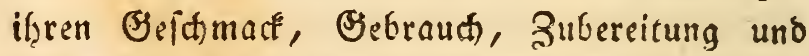
Berwahrung an. Zum âfiucien berfelben wußs. tes fie fith nidft, wie wir, ber Sounde und ber Sdiweine jul hesienen, fondern fie erfannten ithre verborgene SBegenwart aue gewiffen Erhelunngen Des Эoodens, aแz sen Riffen in demfelben, uno aus ber Ejegenwart gewiffer Jyflanjen, bie nuch

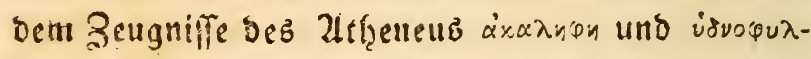

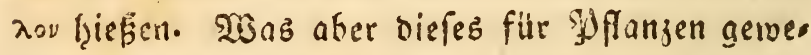
fen, barüber bin id nidtet im Stande eine befries bigende 2lutwort zu ertheilen. Şandel if allers sings aud) foton bamahle mit biefer 2 saare ges trieben worben, weil fie if̧re beften Triffel, fo wie wir noch heut ju Tage, aus bem Jnnern bon Iffifa, aus ben Эiumidifaen woüften erhielten. Die Trüffel bat bemnad Die Ęrre, mit bem গ्रBeinftocf und mit bent Roggen für eines ber äls teften und werbreitetfen Lebensmittel ber Menf(t)en 
unb für cinen ber älteften Şandeleartíel erfannt zu werben, obne jemable ibren grofjen 2unrerth ben ben Tafeln Der Mähtigen un' ber Reiden

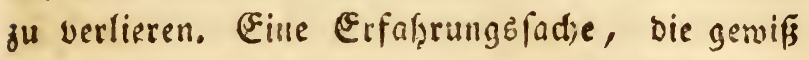
beruubert ju werben verbienet, viele hundert andere minder berülnmte şaaren gibt, bie fie fowobl an Ssüte als an ?iutbarteit iibertreffen. Die Belege der bier abyehandelten (Gefdichte findet man ben Becfmann a. a. D. S. 73 u. $f$

Da bie Trüffeln nid)t, mie anbere 巨屯mäm. me, frifd ju: Marte gebractit, uno auf ber Gtelle verfpeifer, fordern sum Shandel beftimmt, und oft über ein Jab̨r aufbeţalten werben; fo verfteht ez fich von felbft, dafi auth gerwifie Borfichtsregeln zu diefer ifrer 2 ufberoabrung er. forberlich find, ofne weld en fie in Eurjer Zeit berfaulen ober berborren uno berberben milisten. Sie beftehen in Folgendell: Man reinigt die fris fhen aus ber Erbe genommenen Stüffeln mit leis nenen Tühern oon nllem Sdimuţe, micfelt bier. auf jeoe befonders in ein mit mact) setränftes Papier, uno berwahret fie in einem glälernen hermetif(t) verfalloffenen Sefäfie. Diefes legt man fodann in einen 3uber, morin man von 3eit zu Zeit frifdes $2 B a f f e r$ eintragen uno Dab alte ausgiefien läß̈t. Undere tauchen die Trüffeln

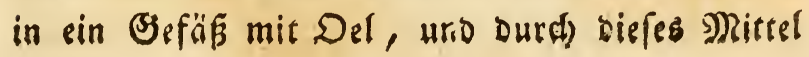


14

bewahrt $m$ an fie ain finerften vor bem nactithei, ligen (Einflup Der \{uft, ourch) welche die Trüffal entweder zu fef̧r auggeobrrt oder zur Sallerte werden würde. Die gewöf̧nlicte uno einfactsefte Theife fie aufjuberoafien beftebt barin, daß̈ man fie in einem unterirrbifhen Serwölbe in ein Sies milcte von Sand und etwas \{eb̨m vergräbt, jes

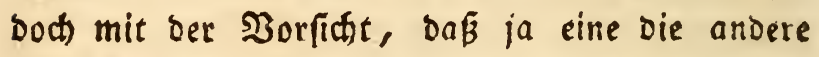
nicht berithre. San muß̧ auch üfers nahlitien, und alle biejenigen wegrerfen, an weld)en faule

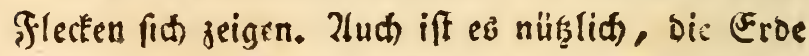
öfters umjumüh̨len ober mit frifcher gu vermechfeln.

Wenn anders, was fidf jeţt noch nidf ente fheiben läft, bie Ingaben oar Schriftfeller nidht -mehrete Irten unter eillem शabmen vermengen: To fino unter ben Europäifiten die Triffein aus Pisniont, aแs Snonterat und aus Maylano die

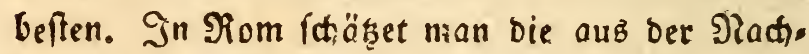
barfhaft oer Graot Norcia fur bie beften. In ben fublicten Theilen oon Franfreich fino fie gar gemein, befonders in \{anguecoc, Jyrovence, Daus phiné, 2lngoumois, Deérigoro, Suienne, auch in Bourgogne, Sorraine, Franthes Enté, Shams pagne. Llm zuignen merden fie ben Reifenden im serbfte in allen SEirthefäufern vorgefent. In Dem Yark von Billatneule, Een Der 2aten bon St. Denif, in Franfreic), traf man fonft eine fo grope slenge Trüfeln an, baß fie Defiroegen 


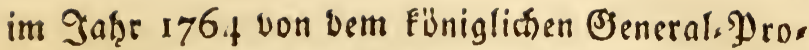
cureur, Seern von Billatneufe, an bie Doft. hänoler in $\mathfrak{Y}$ aris auf fects Sabre, für jäbrlich 250 \{iores und 10 D)fund Trüfieln, die fie in jes Der Gaf̧reEseit biefem Şerrn auछliefern mufiten, verpactet murbell. 2lus allen Triffell in ber 2 Belt werben aber bie 2 ffrifaniften, weldie ithon Dlis nius und Suvenal für die belicateften erElären, nod) jef̧t in Franfreidi für bie beften gethalten.

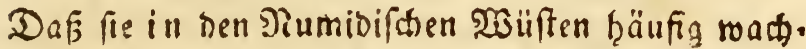
fen, und won den 2lrabern febr gern gegeffen was, den, erzäblt joh. Leo (Africae descr. Ant. verp. 15:6. p. 3c0), Der fie Terfej nennet.

Der gewölsnlidhe Standort der Trüffeln find lidfte, howftämmige Eichenwälber, befonders

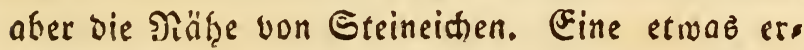
habene \{age und eine Deffnung für ben Regen

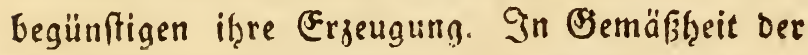
oben angefühţrten änutlictien 2luefage bes \$̧errn Meifter wathen fie in Begenden, wo Eictien,

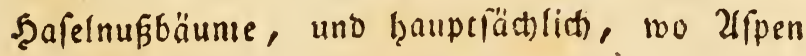
machen, an ber Sord uno 2lbenofeite, nicmables aber an ber Mittagefeite; ill feuchten Boden oder fogenannten futtigten 20 aloboden. Im lichten

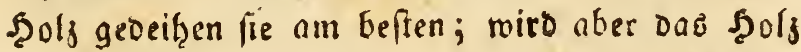
gefällt, fo pplegen fie ausjugeben. Diefe leąte Ers fathrung, welde forwobl in ber angeführten 2lus, age, als wie aud in Den meiften Schriftftellern 
vorËbumt, wilrbe unz faft ben Derbadit einfib. Ecn, die Sruffeln für Glofie ş]rooucte der Baum. murzeln zu balten. Ja man bebauptet fogar, fie

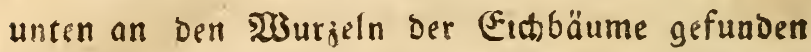

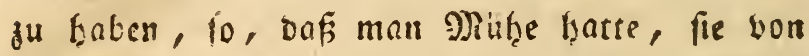
Den Sinoten diefer Wurzeln za unterfitsioen. Man bat enolid jumeilen feinartige R̈̈sper oder Ber. härrungen in oer Sulftang Derfelben gefunsen. (f. Souttuin. Sinn. WH. Enft. 3. Th. I. Bo. ( 53 ). Her alle diefe Enfubrungen bameifen

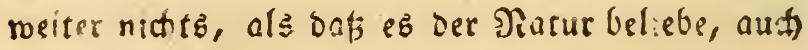
Die Truffel Da, mo es die IInfiande verftatten, lieber an oer Mutterpflanje jaţlojer 'y arajuten, und vorgüglid an ver Sabäbrerinn ber moiften Sctwammarten, oer (sithe, in Bermajarung ju leaen. Dern wenn wir mit diefer die Erfatrun. gen cines Ricteli, eines Brafen De Borch, eio

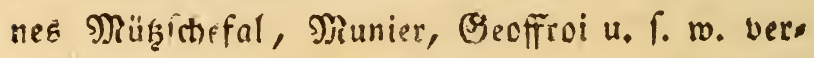
gleituen: io merben mir belefirt, wie behutfam man ben beralsiction Galufifolgen zu WJerfe gee hen müffe, uno mie leiche man in SBegentheil auf Irrtbümer uno Şirngefpinnite verfallen fönne. Diefen leğtgenannten (Semähremännern zu Folge lagt Beckmann a a. D. S. 59 u. f. über bie Stanoorte der Trüffel:

"Diefe unterirrbiltien Scträmme wact)en in einem locfern, fuschtóten, formárjen uno eto was feudten :Boosn, pen nir Rineralogen Damms 
crbe, Stanberbe, Humus, die Franjofen terre franche nennen. $21 \mathrm{~m}$ bäufigfen werben fie in Eichen, Raptanien, uno $\mathfrak{B}$ udienraaldungen gefuns ben, und man roll bemeret habea, bafi fie fid) wenn die Bäume abgetrieben werben, verlieren. linter 2lepfel, Sirn. uno 9iubbäumen foll man fie rogar in 2ingoumcis, too fie felcr ţäufig fino, nie gefunden baben. Dort bält man die, weldie unter Fichen gefammlet werben, für bie beffen;

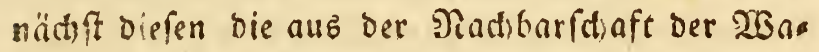
cholberfträuche. Ebenvafeloft triff man fie auch in subeingärten, auch im 2lcerlande zwiftien ben Stoppeln an. Atber im obern Gratien, und viel. leidft in vielen \{änberl, 100 noch nicht barnach gefuctit ift, fino fie auch in mäfig feutten Wies fen, beren Boben aud allerbings Stauberbe, Jumus iff. Sieforeinen in allen \{änbern oon Eus ropa zu fenn, unb fie find auch in einigen (Segen= ben vor afien und afrifa gefundell morden. Sinné fand fie fogar in Sapplano, Rämpfer in gapan, mo man fie eben fo begierig fuct und berfpeifet als in Europa."

S) Man findet fie I bis 6 ?oll tief unter ber Erbe, balo einfam, balo, uno zmar gewöb̨nlid in Slumpen bon 3 biz ? Gritcen benfammen. Eie liegen alzounn aneinander, uno orüten fict flad) an ben Seiten, mit meldien fie fid beriifs. ระn. Zumeilen, jedom filter, berauf jid bie 
3abl ber Stücle in einem lode über 20 Da die Trüffel in Zrofebung ver Tortplanjung mit ocm Sugelthier analog ift, und in if̧rer Sabftanj mehs rere Bererationen von Trüffín entbält, mildie Duct die Zafiöbrung uno Bermeefung oer Nut= terpflanje zu itgrer Trentseit uno Entwickelung ges langen: fo follesn mohl an eincm Dite, wo einft ein Trüffelfeim bingefommen, mit ber Zeit eine greß̉e Menge berfelben benfammen entfethen. 2flo lein es geht hier wie anberkwo in Der शatur; bie formächern müfron im erften Reim unterliegen, samit tie ondern defto vollfommner nerben fün nen! EE fornmen baun nur wenige zum Borfitein, incem die üfrigen entweder frübjeitig verweefen oser vielleidt mit ben erftern zufamm nwadfen. Sar findet zumeilsn Da, mo cine alte Tribfel erft vor furzen verfaulte, eine ganje borut von feftr jablreihen jungen $S_{d}$ mämmen. İerben nun diefe entreder von Infecten boer von ben Schreinen zeritreut, fo mögen fie fich vermehren uno viels leicht in oer indiviouellen 2lnziht fortwathen. Sleiben fie aber in einer Brube benfammen, fo

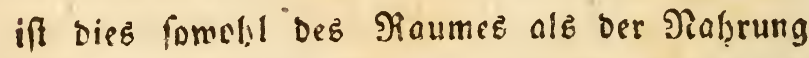
balber unmöglich. Donn bie Trüffel ifteine ben

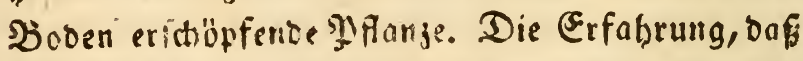
iiber iţr feine andere Fiflanje unmittelbar $z u$ want)= fen pheage, fcheint mett mehr aus biefer llefache hègeleitet roerden ju müffen, als aus bem Ses 
rutbe ber Triffel, ber freulich wohl auth ber fie umgebenoen (Froe fid mittheilet, aber bod nicht verhindert, oafi wenigftens in ber פläţe von ihr viele गffanzen gebeihen.

Man fammelt tie Trilffel vom halben Juguft big in ben Minter. WBenn Stinee und Froft ben Eroboben in feiner Dberfiäctse verfalięen, Donn hat ez mit ber Trüffelärndte natürlitfer $\mathfrak{L}$ beife fein Ende. Ein gelinder Froft ift indeffen berfel. ben nicht nad)tfeilig, er verbeffert vielmef̨r ihren

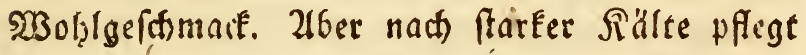
fie zu verweelen und ju verfáminden. Bey gan gelinben Wintern werben baher die Trüffeln bie faft jur Entftehung ber neuen, D. i. bis in ben april binaus, gefunden. Die ähte Trüffel, odẹ

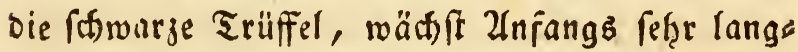

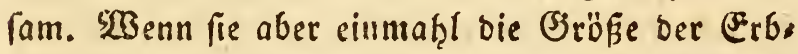
fell erreidit hat, boun foleint auch if̧re Begetas tion leblgafter uno raf́cher zu gedeif̧en. Der Beit punft biefer Sunal̨me fällt in De.l Julius uno in Den 2luguft. (E⿱一𫝀) ift aud) fefor leid)t ju begreifen, warum fie alzoann in einem Monathe mef̧r zuneh = me als vorher in orenen. Denn wenn es auts wirflich in ben früheren Momathen bäufiz regnet, fo ift Doch bie (ErDe nod) nidht hinlänglid) von den Sonnenftrab̨len ermärmet. Nun ift nber die Srüfe

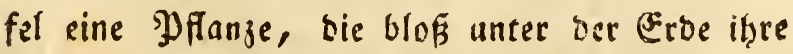
ganse Sebensperivo vollendet. Into da fie Eein 
Jarnint, fonbern sin wahres Erogewädis ift, weldhes nidht burch) Wutjeln, fondern ourch un= mitteibare (Einfaugung an ibrer ganzen Dberfläthe fich ernähret: fo muf fie frenlich woht in jener Zeit am beften gebeibell, wann näḩmld) Die ore ganifchen Theilchen ber Dammerbe, vermittelft ber 20 ärme, ill einen halb gaeartig halb tropf. baren Zuftano aufgelöfer morden *). Es gelïret übrigens jur Sartie, die B̉emetfung zu machen, bie reichfte Truffelärnote fen eine Folge naffer Sommer, und zumahl roarmer Regen in ber letg. ten Sälfte d:g 2luguft. Sn troctenen Sommernt bingegen merben faft gar feine ober boch rur fehr fleine gefunden. Im $9 a b r 17$ lo bat man in gums Sanfrich Eeine Trüffeln haben fünnen (wie offioi berichtet), weil ber vorthergethende ftrenge

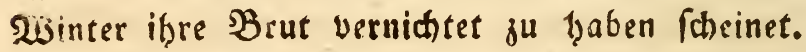
In folden Fällen mag es vielleicht mit ben Trüf. feln mie mit gewiffen Ennandriften geben, bavon man mand)mab̨l viele Jaḩre feine Spur gerwaḩe

*) Es pimmt mit diefer SBetrndtung ganj uberein, dafí bie weipe Iruffel forsobl als ote Sommer. triffel meit friber z̧u ibrer Meife gelangen, als

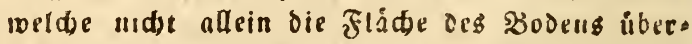
fergen und die frege Goune genieksen, foniern aud wirflud einige wurjelartige Pinịige aufous merlen baberl. 
nimmt, on fie both einigemahl an berfelben Stelle in arofier Menge erfacinen. Bielleictit werden oie erften fieine verbinjert, fid ju entwickeln, uns cine oritte folat unmittelbar auf bie erfte? Die fehr einfat): liutur dieler Degetabilien läf̆ bief won ben Srüfeln fo gut wie bon ben Jolnpen uno manchen andern felpr einfacten Stievern bes Sthier = uno DAanjenreiches vermuthen!

(Seröb̆nlich erreid)en bie Triiffeln die Siröfe einer खelfán ?huf; man hat aber auth Ben. fpiele von vial gröferen. Fauftgrofie find fonon eine grobe Seltenteit, und werben mit 5, io aud

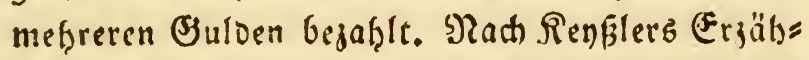
lung foll im Jahr I 729 in Safal eine S:üffel bon I2 Ifunden gefunden uno für 4 louigo'or vers Eauft rorden fenn. Einige gatgre früber foll eis ner Drinzeliinn bon Diemont fogar eine bon I 4 Jfuno uno von ber Grö̈ßze eines Fleinen Tellers überreicht morben fenn. (S. Bectmann a. a. D. (5. 70).

Serr פeifter macht in ber gerüfimten ämtlis wen 2luzlage zu mieberţoblten Mab̨lell eires ro, then Räfers Meloung, wel(her nat) ber Trüffel febre lüftern fenn foll, und ber fie bäufig befudiet, fobald fie ibre völlige Reife erlangt hat, ooer wohl gar bereitz faule Flecken befömmt. Ob nun bieźn ein Attullabus, ein Carabus, eine Cicindela ober wohl gar ein Acarus fent, Eann id nicht bes 
frimmen, ba idf bas Infect niemaf̧l gefergen han be, und ba in berfelben 2 ublage aud nicht ein. mahl bon ber Srö̈fe biefes Infects eine Meloung gemadt wirb.

Ran fammelt die Trüfreln bom Enbe 2 (u.

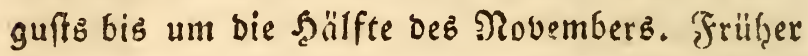
whroen fis gुu Elein utb aud weniger fomacthaft fenn. 2tllin bis vorzüglichfte Hrfache, warum fie erft baun gefammelt werben, ift moht ber Duft, burd) weldien fie fich erft bann ben' Sammlern und if̧ren Behtilfen, oen Sunden, verrathen. 200er ben Serud ber Trüffel genau Eennet, ber fann um biefe 3at, bey warmer 2 Bitterung in ben abenoftunten uno wenn bie luft if̧u fanft entgegenwebet, bie Siegenwart rines Neftes bers felben auf 20 Sthitte weit wittern. FE gibt leute, welwe fie feţr verläfing ju fuchen verfteţen. Frenlictwobl ift es weit fáwerer, in einer Ser geno Trüffeln zu fuकen, wo nod) niemahle wel = d)e gefunben worben, als wenn man bereits mit if̧ren Gtanöbtern befannt iff. Zllein biefe \{eute wiffen es fehr gennu, Daj̉ ber Drt, wo Trüffeln gebeifen follen, etwas erhaben, ber Boben leicht, etwas fandig und jiemlich farwary, bem regen uno ben Gonnenftrahlen geöfnet, uno ourd) bas

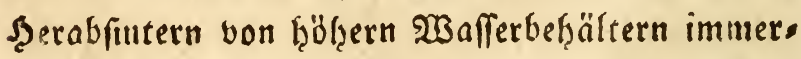

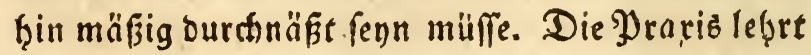
lie folde nur in liabten bodftämmigen 2 älbern 
bon to bis so gatiren su fucten, hie nur menig ober a far fein llaterbois baben. 2Bo viel Mocs oder Rräuter den Booen benecten, da koffin fie Eeine Sriffelärnte : geminnen. Sie mögen mohl fogar an Zusfehen ber Bäume, Eifonders in oer BSegeno der TBurzel, gemiffe Meremahle ahn. bell, bie fith aber leidter ourch bie llebung erfer, men als befthreiben Iaffer. Mian mill beţaupten, baßj es gemiffe गुfanien gebe, aus welchen firth diz Siäbe ber Trüffelnefter errathen liefse ${ }^{*}$ ). allein bizljer babe id now nienable in Erfabs rung bringen fönnen, was für 2lrten diefelben

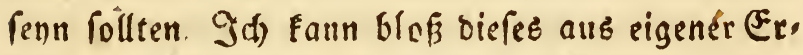
fab̨rung angeben, baj ich in ber פählge gefunbes ner Trüffel Cistus Helianthemum, Bellis perennis, Gnaphalium dioicum, Myosotis sylvestris, Viola arvensis, Anemone sylvestris, Inula hirta, Aster Amellus, Lychnis viscaria, Polygala vulgaris, Genista Germanica, Asclepias Vincet oxicum, Prenan-

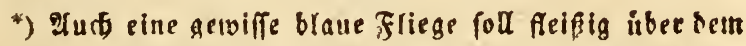
Drte fowpben, und baiurds bie Begentbact bee Triffel verratben. Bermuthlid leat fie ibre Evee Dabin, Damit isce 33 at in derfelben ibre $\mathfrak{N a b e u n g}$ findell móge. Dee (Sraf von Bocd) bat ziney fol. Wer Iriffitfieaen unter dem $\mathfrak{N a b m e n}$ mouche - de Truffe abgebildet, cine braue und eine fdowarze. 
thes muralis, Lapsana communis, Hieracium sylvaticum, Potentilla alba, Orchis Morio u. ugl. Geobachtet habe. 2lm näctiften ba. bel) fetien mir immer ein Thymus voer eine Tormentilla ju fenn. Der Boden forobr als bie Stämme maren in anfebnlidier Sienge von allerlen überirrbiften Sttrömmen, befonders aber von Jilflingen und Täublingen aller Zlrten gejieret. Daz geübte $\mathfrak{A u g}_{\text {ber }}$ Trüffilfucher ent. beckt mit \{eichtigfeit auf 3 bis 5 Fuf Entfer. nung bon ben Stämman ber Bäune gawiffe Er. böh̆ungen, bez Erobobene, weld)e von benjenigen, fo die Maulrürfe *) ju macten pflegen, ganz und ga: berfhieben fino, indem fie Eeine ourch. wüblte Erbe an ben Tag Gringen, fonbern nur eine geringe zuweilen bon einigen Rifien beseid). nete Erhebung ber oberften Rinde oss Bobens barftellen.

Zllein es gibt nod anbere Mittel, biefe wer. grabene Gääze der Pomona ben verborgenften Ehlupfwinteln zu entreifen. Da bie Sthmeine

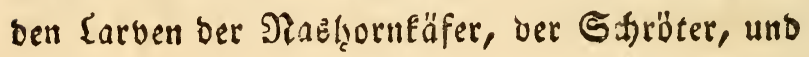

*) In Geaendell, tro es viele Ersmáafe, Walsrat. ten, Somfter oder M?oultourfe giebe, da ift es nid)t gut 'eriffell zu fudiell, denn diefe Tbiere pflegen iguen nadguftellen und fie nuszurotsen. 
ben Regenwürmern (ber fogenannten llntermaft)

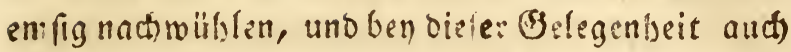
manchmahl Trüffeln hervorarbeiten: fo ift $\mathfrak{e E}$ (man meif nicht beftimunt mann?) ben liebba. bern snolich cingefallen, fid ifrer zum Uuffucteu Der Srüffeln z" bebienen. Sermuthlich waren bie Italiäner bie erften, welche fith biefer Intethobe bebienten. Platina, melder im Gaḩr r 4 s I farb, fagte fotion (in reinem sofue de honesta voluptate, von dem Şaller Biblint. B. I. p. 235 S2actricht gibt), fie würoen mit Säuen gefudi)t. Diefe Eerfitsung mag bann ungefähr um bie Nitte des isten Gabrhunderts ifren llrfprung gebabe haben. In Franfreich, befonberz in 2lngoumciz uno Derigoro, wie aud in Dberitalien pflegt man noch beut zu Tage die Trüfreln auf diefe TBeife zu fammeln. Die Shweine, weldhe baju gemäblt werben, müffen ungefäls 5 Monatbe alt, (क)Inne, uno jum Beb̨en gewöhnt fenn, um bie 2 rbeit vom Morgen bis zum 26

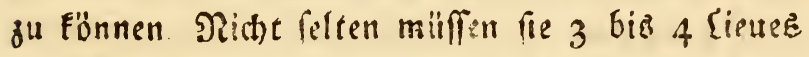
in einem sage burchlauren. Efben befiwegen bleibt ein Sdyein ju biefer Ifbitht nur ein Sabr taug. liđ), uno jährrlich mußz ein anderes dału abgerich. tet werben, welches aut) nicht viele Inube macht. sMan fuht ein folchez aus, welthes Truffeln bes gierig verfdlucft; benn mand)e freffen fie gar nidft, und biefe fino auch jum Suchen ganj un. 
tauglith. Tene fübrt man in Segenten, mo Trif, fell fino, over wo man Dergleichen vergraben bat-

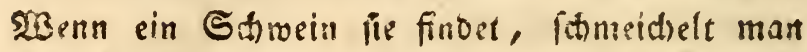
Demielben, uno gerwöhnt eह, feinen Funo geaen (Fitheln ober ein arberes nocs) angenebmeres futs ter fabren ju raffen. Das Suchen unit fold)en ab. gerictitsten Säuzn gebt am beften ben guter 2 Bito terung; nitht bey farker Niaffe, auch nicht bey beftigem winde. Dagegen ift ein gelinder $\mathfrak{W}$ Bind gut, uno albonnn fübrt man bas Thier gegen Denfe!tere. Sat es eine Trüffel gefunden, fo greift man if̧m ans $\mathcal{O}$ h̨r, jief̨t es juriick, uno nimmt jene mit der Sand heraus, morauf dem Sthreir

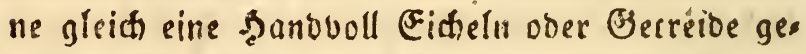
geben mird. Weil die Säue aud felpr begierig nach Der fogenannten llntermaft röblen, fo muß ber Führer fo gefdicft fenn, fie bavon abzuhal. ten. Er räumt aut bie Sicine hinmeg, welthe ben Shieren fobabll fonnen; benn oft frito fie fo hisgig im Sud)en, dafie fie fih ben Rüfel ganj runo uno Glutig wüblen. Un 2Boronien, Floreng uno in andern Segenten bon Italien foll man ben Säuen am Şinterfufie sinen Stricf binben, foldbe bor fith berlaufen laffen, uno fie, menn fie zu bres.

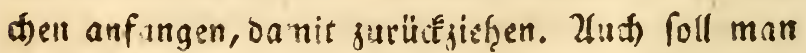

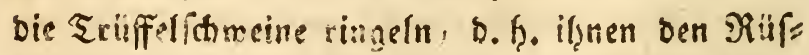
fel mit einem lebernen Rienen belegen, Dill man, senn fie sruffeln gefunben baben, abnimmt, 
morauf man if̧nen (Eid)eln ober Raftarien zur Belol̨nung gibt. 2ulfo ift es rebr notbmentig, baßj man in Segentien, welche Trüffeln haben, viift) ben Schmeinhirten mit ber Seerbe fommen läft, als melche alles aufjef̧ren mürbe. (BBeff= mann a. a. D. S. 64 u. $f_{t}$ )

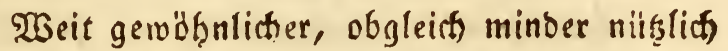
ift aber, jumabl in Deutftland, bas 2uffuctent ber Trüffelı init Şunten. Diefe Art ber Trüffels jago facint indeffell jünger zu fenn als bie mit Den Sdincinen. Nach Deutfoland fint bie erften Srüfelfunde im erften Biertel bez vorigen Sahre ḩunberts, jugleich mit Trüffljägern, aus Itas lien verfarieben morben. Diefes grofe Berbienft wirb verffiedenen grofiel Werfonen zugeeignet, und vielleicht find metrere fait jugleich auf biefen Einfall getommen. 2luguft II., R̈̈nig von Wo. len, foll ums galir 1720 zehn Tritfelhunoe, bas Stück für 100 Thaler, aus gralien baben Eom.

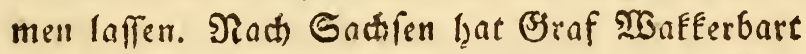
im gabr I724 die exfen verfarieben, natbem

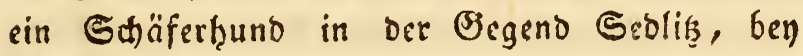
Dresben, im Dctober 1719 Trüffeln entbeck hatte. Jm $\mathfrak{B}$ randenburgifhen erbielt ein Italiäs

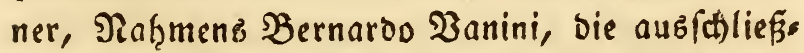

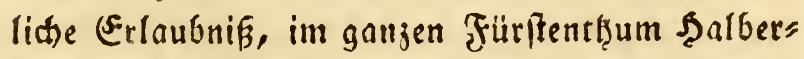
frabt srüffeín aufzufuchen, Dagegen er jährtich

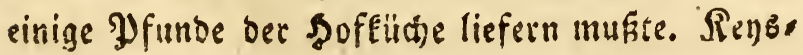


ler meinte, ber 2Dittembergifdie gefreime Finth bon Forftner habe in Deutíblano die erften fus then laffen, burch bie benoen abgerichteten feun. De, welche, auf feine 2eranlaffung, Dem Erb. prinjen boll visttemberg, den er als Dberbof= meifter auf Reifen begleitete, am Turiner \$ofe gefthentt twaren. (Bectmann a. a. D. S. 7.8 น. $f$ )

Man fann nllerlen Racen von Şunden zu

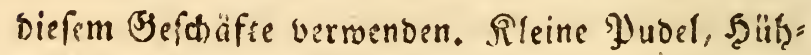
nerfounde, Spiģe, Bolognefer und Dahbhumbe fönnen die nähmlidien Dienfte leiften. Fs gibe aber mehrerley smetboden, diele Thiere ju bent Triffelfuctien abjuridten. 3. (5. man läß̈ fich rine S ïffel vom şunbe apportirell, bie aber in Scinmano eingenähet feryn muf3, bamit or fie nicht freffen lertie, als weldie llnart f́dfmer wieber ab. gugemöbnen ift. Sernach geht man mit betn Şun. De aufs Felo, verftect die Trüffel leicht, läft lie futten und bringen, und fo lernt et bie Runft Ealo, to baf man iţn im Serbfte in ben fübren uno ernftlid Trüffrin futhen laffen fann. Die Staliäner, meldie die Trüffelḩunde gewöh̨n. lich Nutta nennen, geban ihtren bes Morgenz ein Stüd Bron, melches in Trüfelöhl gefocht wor. ben, uno jiehen fobann mit if̧nen auf bie Jago, wo fie bon jedeemahliger Meloung mit Brod be. lob̨ut rerben, fo wie fie überhaupt nichts anbers 


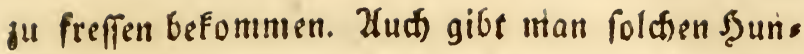
ben öfters in Butter gebacfene Trüffelfiältten jum Futter, und wenn fie diefe Poft föäz̧en ges lernt haben, fo begrabt man im 20 aloe eine ges bactene Srüffel anfangs fehr feitit, nad) und nact) aber immer tiefer, und läje fie futhen. So gea wöhrnet endlith ber Trüffellzund oen Serud, uno (c)arret aud jebe andere auk. (Fin guter Trüffels ḩund follägt an ben einem jeoesmabligen Funt, wie auf einen Şir/den. Sian muß nun berbeneis len, benn fonft wiro er viele Stücle verftiarren unt verberben. Man gibt bem Şunde fein Futter, liebfofet itgn, uno grabe mit eitrem gewöbnnlichen Bartenfpatel die Trüffeln felber aus.

In bes Erafen be Phord Letres sur les Truffles du Piemont, Milan. 1780. in 860., Bulliard in ver Histoire des Champignons, Paris 1791. Fol., in ber 3ritung ber Inouftrie uno Epeculation, swien 1804 in 800. , uno in

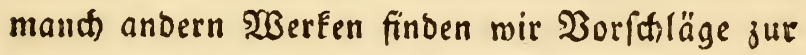
Eultur der Trüffeln. Da ich aber in allen biefen viel llnanmendbares und Fingebildetes finde, fo will ich an bie Stelle berfelben lieber meine eiges nen vorlegen.

Man erwähle ein ab̧ängiges (f̧hiefes) ges gen 20 eften geneigtes Stüd \{and. In Deffen obers ften Theile wird ein von Bäumen befhatteteb

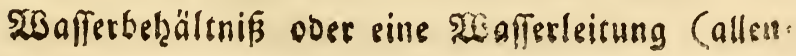




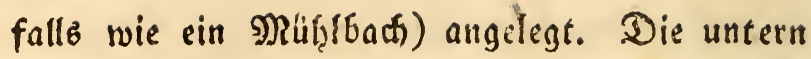
Theile bes Şügels mögen einzeln errad)fene (E) (hen, Raftanien (Castanea, nit)t Aesculus!) \$Badbolberbäume u. ogl. befhatten. Der B̉o. Den fen leidht, fifward, locker unb fanbig. 2lls lez Sebüh zen, Gefonders aber bie ftark wuthernben Eräfer, wie Renlggraz, Binfen, Sunderoggen u. Dgl. müffen vertilgt werben. Defteres Beftreuen mit Zfiche iff fowotel jur Bertilgung ber Moofe aís auch aud anderen Urfachen befonders zu empfeh. Ien. Berftreute grö̈̈ere Stilcfe Ealfartiger Steine fino nidte allein unfwäblith, fondern fogar ferce

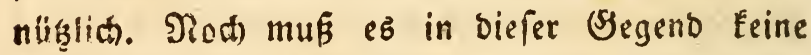
Mäufe uno Éeine Maulwülfe geben, und das Land muß niemahle zum Sumpfe werben. Man fucht bann im 2(pril ober Man eine junge frifte Brut von Trüffeln, jertheilet fie, uno legt zmen Doer bren Stüffwen jufammen 2 Zoll tief unter bie Erve, jeood mit ber Borficht, baß immer eine gute ภुortion ber mü̈tterlidfert Erbe an ben jungen Trüfeln fell Eleben bleibe; wefbralben es

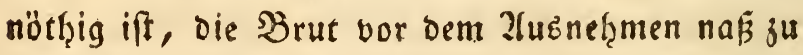
macten und nafi ju verpflanzen. Man hat nad). her nichte meiter zu tlsun, als das itber biefen Stellen aufécimenoe llnfraut fleißig zu vertilgen, und oafür ju lorgen, baf es Dem Baffein nie, maţls am 20 affer inangeln mäge. Frenlidwoht 
ift dieß Eeine Setḩode, die man fo leitht überall, wie die Culcur des Elgampignons, anmenoen föunte. Unterdeffen gitt eह boh) SSegendan genug, in we!d)en man eine fold) Truffelplantage anlegen Eönnte, ol̨ne eben großie UnÉften bacauf ju vers wenben. Bielleidit Ëbnte man aut Treibbeeten unter Slazfenftern anlegen? Iक wärbe rathen, die Saju varmendete Erte mit vielen halbvermits terten Theilen bon 22 aumrinden uno abgefalle. sem \{aube mit Dünger von $23 n$ torhieren, vor, züglith von St,weinen, wie aud von allerlin Infecten u. ogl. ju mengen. Das Beet müßjte erf̧aben angelegt werden, samit die હonnenftraf̧. len nicht blopis die Dberfäh), fonbern auth die Ränder berfelben ringsherum erwärmen Ënnen.

Mran hat nud SMittel fie zu bertilgen vor. geftlagen. Das నachlucten uno IImwühten der. Srüffelfucher, melde burch ibr (Seldäft man= bem Srumbeigenthümer Shooen jufïgen, war bie Seranlaffung berfelben. 2llein id) halte es für unnöthig, bergleicten zu wiebertiolen; benn in gebnuten Sründen wachen Éeine Trüffelı, uno Die übrigen (iold)e nähmlich, beren Boben nich nöthig hat umgeronbet zu rerben!) Fönnen surth Umzäunungen beffer als burkf bie unfidere uns zugleict) verfthrenderifite 26rt Der 2lubrottung ge' fiblizget werden.

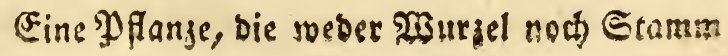


hat, unb bennoch kein Parafnt ift, beren äufere liche Beftalt einen mineralifthen Rürper nacholys met, beren Sufftanj hingegen ourch uno ourch sin pures 2lggregat von eingefchndtelten Reimen barffellet, ein foldes ?aturproduft ift in Der Shat cine meremüroige uno fehrs säthfelbafte Er.

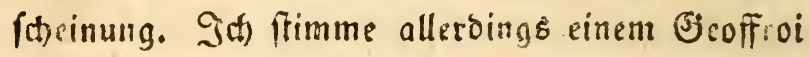

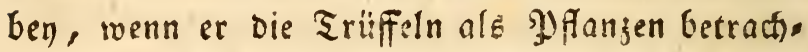
tet, welche zugleich Wourzel, Stamm uno Frucht fino. Эat bemerfe noth an ifinen in ben Verties fungen iherer $\Re$ unjell um und um gerviffe Filjare tige fïrper, welche vielleiht die Raf̧:ung wie

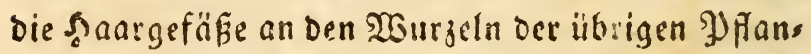
jen einfaugen, uno mit bem weißen Marke Ders felben in Berbinbung fielsen, das fich in fhichts förmige \{abyrinthifhe \{agen jroifhen bie 2 bern Der Reime zerftrenet. Diefes Markidite 2 Beefen biloes gleichfam fo vicle eingelne Riötchen (oaher auch in Der Deerfiätie bie Snotiate Drufenför. mige Beffalt ber Trüffel!) uno wenn diefe Rröts wen einen gerwiffen Erad der Reife erlangt has ben, fo fangen fich in bem Mittelpunfte eirez jeben berfelben bie Reime zu biloen an. Te ftär. Eer, je foftuoller bann bas Fleifh der Triffel in Der 3ait ber Entwittung geworben, Defro melpe rere Pitime werden fir in Der Jolge in Den Mit. telpunften Der . Rnötchen abfeşen, Defto größ̈er, refte bollfommner wirb bie gange Triffel geocis 
hen. Denn on bie Reime viel wäferiges $\mathfrak{W e f e a t}$ zwifchen fich baben, uno fith inezemein ben fphb̈. rifhen Eeftalten näbern: fo milffen fie natitrlich. Den Siaum bez Banjen Geträchtlich vergrö̈ern, da daz MarÉ vielmeţr aus faferictiten Drgan:n zufammengeweft ift. Soll bemnach eine Triiffel borzüglid grof meroen: fo mus fie nicht allein zur Zeit ber Reife von Bärme uno Feuthtigfét begünftigt merben, fondern fiton ifre erftes Ems porfeimen muñ das Bepräge eines Eraftuollen Stammes an fich haben, und Éeine wibrigen Esin.

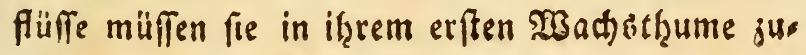

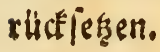




\section{Die meifße Trufficl (Tuber album Pers.)}

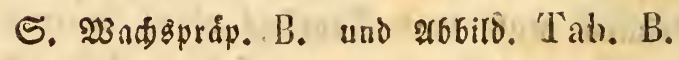

5)? trïffel im Begenfake ber vorigen, reíche auch bie Serbft, und Wintertriiffel genaunt wirb. Eigents lich wiro fre zwar nitht im frühlinge reif, jebodf immerhin, im Durdfhnitt, um ein paar Mo. nathe früher als bie vorige. Nan finbet fie aber auch viel früher, weil fie nidht unter, fontern über ber Eroe zu madfen gemobnt ift. Im Früh. linge, fagt B̉ulliard, füunce man fle mobt eher für was immer für ein anderes 2 egetabil ber Sdraammfamilie, als für eine Sruffel halten. Denn fie if bamabis nod) ganj weich, glatt, uno von reiner meifer farbe. Erft, mann fie

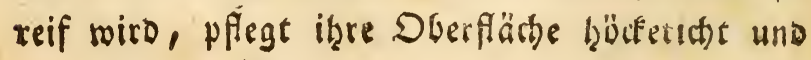




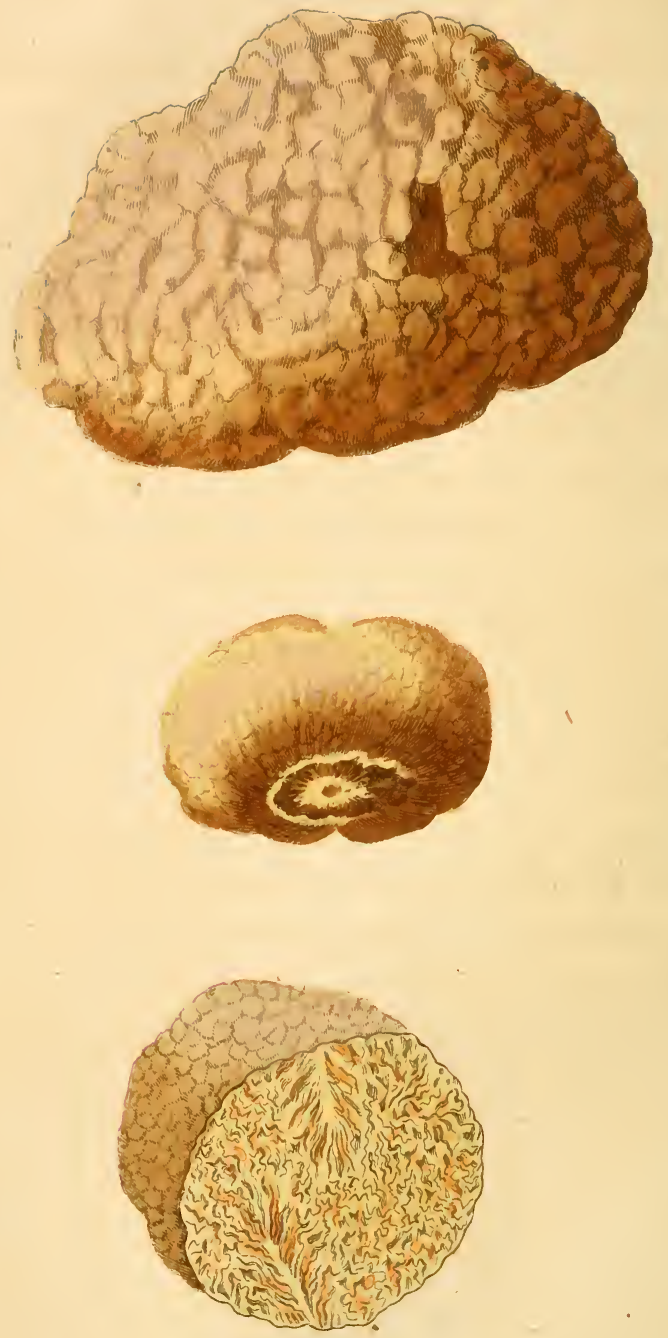

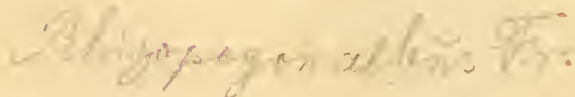

Tiliere allown. P

Clir ineipe Srüffel. 
Elof̧räunlich ju meroen. Eichat jeoow nicmabls foldie intbige und flachleitige Erbabenbeiten, roie wir fie an der äitsen Truffil becbactitet haben. Shre Schale wilo auch niemahls to hart. Sigre

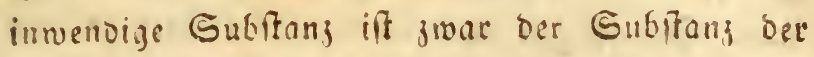

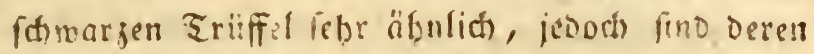
210er: won Den Sdmammfeimen uno die Sthit). ten beg glenfeg viel feiner und von einer ange. nethmeren rötblichen Färbung.

Die meifie Triffel bat jroar Écine Sasurgel, nber sod) einen (d)eibenrunden nurjelfürmigen

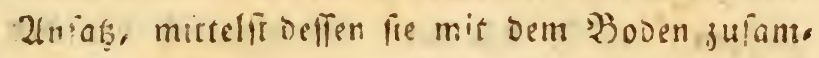
menbanget uno aus osmeloen ifre Sabrum ein.

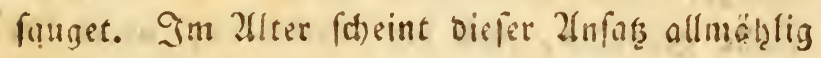
zu verifowinden.

Da die meife Srüffel bizher faif allgemein entweder für eine thofe 2lbart, oder mobl gar nur für eine mintier bolleniete Miodification oer

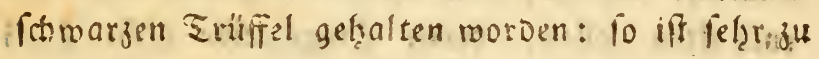
vermuthen, daj das I)ecite, was von diefer ges fagt worden, auch auf bie meifie angemendet mers Den fünne. Die meis: Triffel ift indeffen reit wea maer idmatfhaft, ibr Seruct iff etras unanges netrm, uno fälls ziemlich ftarE in oas Rnoblauchs artige. Sic foreint indiffen viel filtener als die

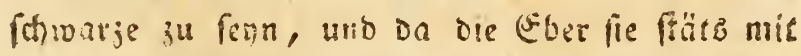
gröfser \{lifternbeit aufiubsen, aud biel leidfer als bie fobronrjen entosten, incem lie die fyähs

(5) 2 


\section{6}

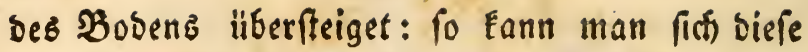
ihre Seltenheit um fo viel leichter erflären. Im Iller werden Diefe Trüffeln graubraun, und fes hen faft wie cin Tugelfánamm (Lycoperdon) aub. Sie pflegen auch itterbaupe fleimer zu fenn als bie fhroarzen, und ibre Beftalt ift im Ban. sen regelmäß̈̈ger uno rünber. Man findet fie meţe

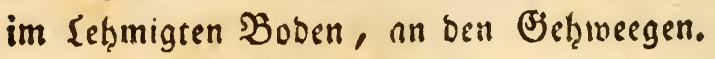

Im şandel if biefe Trüffel ungemöhrnlia. Man iffe fie marinirt, in Spalten jeridinitten. 2lis Sugabe ju Fleifthbrüfen bebient man fitt ber. jenigen, welde fich burch Wohtgeruch vor ben

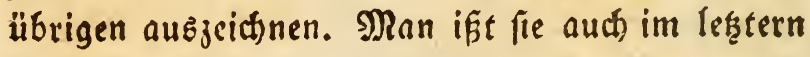
Falle gebraten, wie bie Raftanien, unter ber Gerbiette. 2aber es gibt aud ganz geructlore Stücfe von biefer 21rt Trüffeln. Sie find übris gens forwohl als bie fofroarjen eine etwas unver. baulidie Speife, von weldier fid alle biejenigen enthalten follen, beren Eingemeite nidit gans nach) श्ञunide verbauen. 

III. Den Saiferling (Aimanita Caosarea P.)

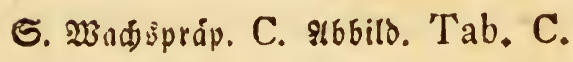

Der Raiferling ober Serruffiwamm ift ber evelfre son allen ebjaren Stwämmen. $\mathfrak{O} 6$ er wirfe

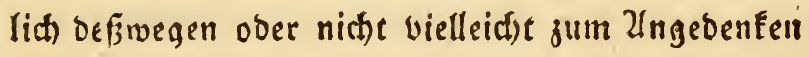
Deb unglüctlicten Tiaifer Elaubiub biefe to erthas bene Benennung erţalten habe, if frenlid) woht now bie Frage. Ess nennen ifgn zwar meţrere alte Sdrriftffeller ben Rönig ber Sdwämme; uno in Der That fifeint er biefen Piahmen forobl burd

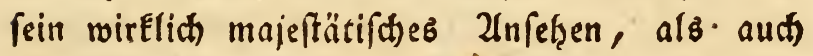

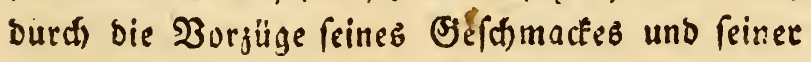
übrigen Eigenfthaften zu reditfertigen. Unterbeffen iff és bod eine Thatfadt), bie in ber Beffidite biefes Stwamm: errö̈h̨net zu werben verbienet,

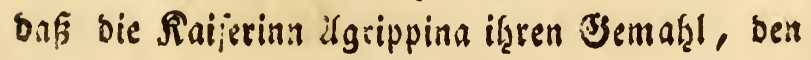


Rïmifonen Raifer siberius Slcubius, melder cin befonders grofer Freund diefer Epeife gerves fen, mit einem vergifteten Seridte dieles Strom mes bingerithtet habe, um iţren Kiebling Domio tus Sero, einen Stiefrohn deg Elaudius, auf

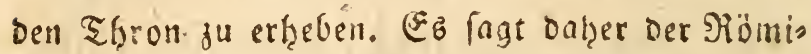
fide Dichter Invenal in der Sar, Vca:

Vilibus ancipites fungi ponentur amicis ,

Boletus domino, sed qualem Claudius edit,

Ante illum uxoris, post quem nihil amplius edit.

utio in ber Sat. VIta:

_ - - Minus ergo nocens erit Agrippinae

Boletus; siquidem unius praecordia pressic

Ille senis, tremulumque caput descendere jussit

In coelum, et longum manantia labra salivam.

Lind S)?artial binterliefz uns folgendes biecauf bejogenes Difficton:

Quid dignum tanto ventrique gulaeque precabor?

Boletum ut, qualem Claudius edit, edas. 
Eben fo pflegte auth, raut bem 3eugniffe dez Suctoniuz, oer Raifer Siero feloft über dies fen Sibramm fareaftích ju foberzen, und ben Bsletus cine siöteripeife fu nenuen, weil fein

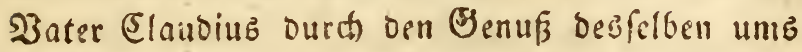
Leben getommen, mo weil ez ben ben Siömern Sitte war, die abgefhiebenen Raifer unter bie Sötter zu zäblen.

Die アïmer pfiegten näbmlid diefen Sthramm, welt)en diefe fo berït)tigten G(t)melger mit einer gailj befonderen Jubjeidinung beehrten, Boletus zu nennen; sod heifter aud benm Plinius Volva, und Cicero hat von il,m unter ber Benen. nung Elvela gefproctien. Die beutigen Fitaliäner ber Segend von Rimini Eennen diefe Schwämme unter Der Berennung: Ovoli rossi. Ju Toz. canif(t)en fingegen beifit ver Raiferling: Uovulo ordinario. Im Franjöffaten wird er l'Oronge vrai, auci l'Amanite Orangé, auch le Jaseran, le Laseras jaune, in Languedoc Roumanél, Dorghe genannt. Luf Şulländifth beifit er : Gouderverwige Kampernoelje, auf Dän. Den gulofarbede Blabfuamp, auf Sdrwedift : Sulofärgabe Slabfoamp, auf Enngl. The golden Agaric, Epan. uno Jortug. Agarico caesareo Ur gomba (Dominorum fungus) bief biefer Sthramm, wenigftens ou ben Beiten Des (s.ufiug, in llngarn. 
40

Da biefer eble Stframm mit bem giftigften aller Sڤ̆vämme uno mit vielen andern fä̈olio

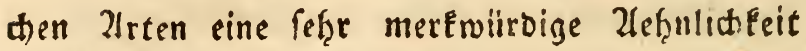

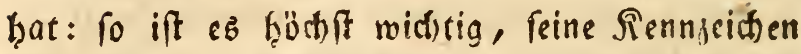
fefir geriau in Seö̈htniffe zu behalten. Taft alle bie übrigen Zirten ber aus ber Erbe hervorwat). fenoen 20 ulfffirwämme (Amanita) fino giftig. Ifls efbar ift wenigftenz nufer bem gegenwärtio gen fein einjiger zu empfeb̧len. Ifm leidteften möct) aber ser fiaiferling mit bem gemeinen flies genfwroamme (Amanita muscaria) berwechfelt werben; befonberz menn, wie es b̈fterz gefchieht, auf ber Dberfiäche dez Şutez häufige \{äppthen, als Ulberbleibfel ber abgeriffenen $\mathfrak{x}$ ulfthaut, bäns aen bleiben, utb fie wie jenen feinen unächten S3ater mit getäfeltem Ed)mudiner verjiersn. Ull: in oer fficanforwamm hat einen Durpurro. then şut, weifse Lamellen, einen vergängliánen Sing uno eine foft aniliegende in Flocken fich zero pflitc ande Mulfiraut.

Die vorzüglichiten Rennzeicten bez Raifers Irgee find semnach ein grofier meifier weit geöffs neter Wou!f, pin Enolliger, voller, von aufien Oafrgelber GtrunE, ein groker berabhängendet Ring, ein labaft Wumeranjenfaroiger oder ounfels gologelber Sut, beffen Mitte etwas niebergee

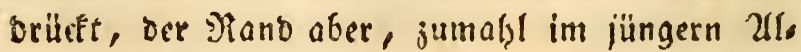
ter, mit Strahlenartigen Falten gejert ift, blof. 
golbgelbe, fef̧r breite \{amellen, und ein ïberaus feines, meiches, meifises fleifh. (seruch hat er resing, uno diefer hat einige deñillichét mit bem Dufte des fliedero (Syringa vulgaris).

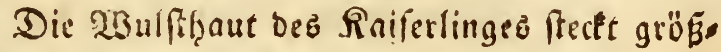
tentheile unter Der Erbe jwifhen Dem Moder von nogefallenen Laub, Baumreifern, Moofen, Flectiten uno Srätern; uno ba fie nicht felteniin ber Erbe frecten bleibt, wern man ben Stfwamm berauejielit: 10 muf man fich baourch nicht irre maden laffen; Denn ia babe eben befirmegen mef̧s reve vefentlictie fiennjeiden angegeben, bamit, im Falle bas cine ober bas andere mange!n möch. te, noch immer genug andere übrig wären, woraus man fich von ber Jfedtheit oicfer Strommart \#̈berzengen Ëbnte. Die Fa:be iff überhaupe bas

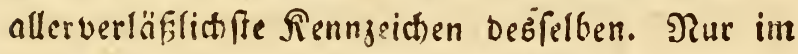
Ieteten Ulter wirb es Bronzfarben over braun uno Flecfig. Dann ifir er aber aud nicht mifre genuts. bar, weil fein weiches alsoann gelbliches Fleifd

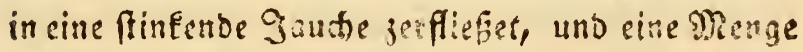

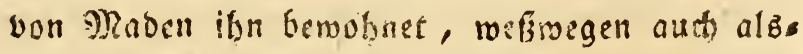
tann fein Strune nitht mef̧r voll, fondern ţoh̨ uno jerfreffen erEannt wiry.

Im erften Buftande ber Entroicfefung gleitht er einem Ey von weiflicher farbe, meldiez jes bud etwas größzer und oben viel dider ift als das

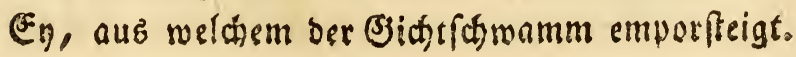


Balo barauf zerplatgt es an feiner Epitge, uno Der Dottergelbe Sut orängt fich hervor, moran nuch jumeilen, weil er in oer Jugento etwas Eles bricht ift, balo mehr balo weniger Stückcten bon Diefer Salut Eleben bleiben, wie biefez ben Dem Fliegenfarwamme viel öfter ber Fall ift, uno diefe reißen \{äpphen verzieren ifgn auf eine gan eiges ne wisife, Eobalo Der Scut ganj aus oer Seöhle dez Evyes (ribulitę) bernuz ift, fängt er an, fid) auszubreiten. Eine natürliche Jolge davon iff, Dafi fich Die untere Fruchinaut (Epicarijum in-

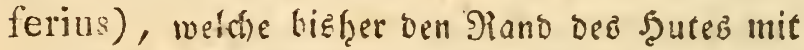
Dom Etrunfe verbano, uns bie \{amellen verbarg, um uno um van bem sute losreifet, uno ba fie fiäfer als nn anoern Etfrömmen ift, fo bleibt fie lange Beis als ein anfehnlich gefaltener ging an ibrem (Seburtzorte fithen, und neigt fit) nach abrörts. Sie ift gelolid) wie ber Strunt uno die \{amellen. Im reiferen Ifter läfift fid auch bie vbere Fruchthaut won bem Scute leitht ablöen. Dann bemerft man zumeilen audh eine gewilie feine gleidhfam gewirfte Faferngeidnung auf ber Dberfläche bes Şutes; und nicht felten zertheilen tiefe Spalten fein Fleift) bie zu ben samellen. Die 26 änderungen. beftehen, nufer ben Mros bificationen, in ber felcr mandelbaren Srö̈ze; benn an Söbe varirt er von 3 biz zu I 4 3oll. Der gröfte Durtameffer Des Sutes war mir auf 
10 Soll bemerfbar. Der Shut bfeibt mantsmabl etwas länger geroülbt, wird aber am Ende tm.

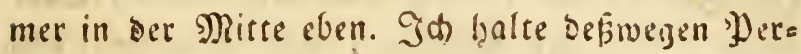
foons Amanica caesarea uno $A$. aurantiast nur fïr cine Specicb. Sonft mäfte ith nur die bon mir folfft brobachteten, auf einer Stelle bens fammen wachfenden, Durd) llebergănge vereinigs, ten Indiviouen für bende ber iben genannien 2 r. ten erflären.

2luf Den Marftpläßsen Wiens habe ith ifg now niemalgle angetroffen. Dinnot) fano id) ibn fowolit in der Sä̈be als andernärts. Dorjüglirb

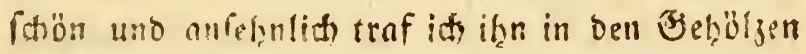

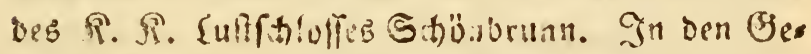

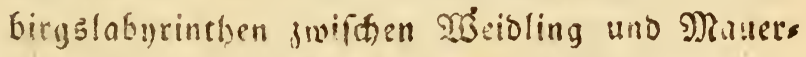
back ift er eben nidhe feltiam. In Mäbren, Ilns garn und an ben Rüfen des 2foriatifक?n Meeres wiro er bäufig gefammelt uno getroffen.

Man rübmt jum Siebrauch nur junge derbe Stüce, unis natioem man folche mit $23 a f j e r$ rein= lidi) abgefpiblat, bab linterfte reggeftuitten, uno die camellen abgrlopet: fo zerfotneiset man Den ganzen Shroamm in Spaiten, bercitet eine Brühe von quter Fleifoluppe mit Butter, Mebl und jerffofiencm sobaijenbeod, nimme tann auch

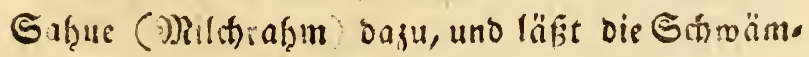
me bumit gar werben. Manthe lieben metor ben

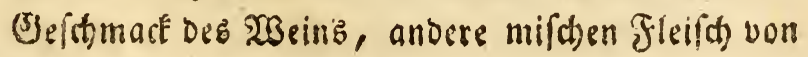


jungen şüf̨nern, Jröfhen ober Filfhen baruks ter. Zur Wö̈rze bebient mall fith balb ber Sar. bellen, balb bez yyfefferz, ber ŞerwürgnelEen, Der Muąatenblïthe ober ber Sitronenifhalen. Zud Duendel, Majoran, Jeterfilgen und Zmiebeln

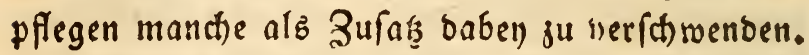

Der Faiferling wilft bie Seridite fo ftare, Daß3 man eine grof́pe Duantität Safran antenben müfte, um eine gleithe Wirkung hervorzubringen. Elufiub erzähletet uns nađ feiner cigenen nais ven 2 beife, er habe sines Tages bey einem llne garifaten Magnaten, Mahmmans Balthafar von Batthyan, auf beffen wohlbefeftigtem Sdiloffe Nemeth. Srgyinar geipeifet, als wohin er alliähr. lith einige Mable burdh ein eigenes Stiff von 2Bien zur Tnfel fen abgetiolet worben. Da nun Die Raiferlinge in if̧rer $\mathfrak{B}$ rühe aufgetragen mut, ben, er aber vorbem nod) niemahts fie gefpeifet batte: Fo funnte er fich nicht enthalten, ben cbs Ien Safffretnd in franjöffifer Sprace anjure. ben (benn, fagt er, biefer grofie Mann fprach) auser feiner Mutterfprathe auch \{atein, Staliä. nifd, Tranjöfifक, Spanifh, Deutich uno $\mathfrak{B a n =}$ balif(t)!), uno ifgm faine Bermunberung zu bes zengen, daf man biefes Eseridt fo gar fef̧r mit Snfran gemalizt habe. 2lllein ber edle Woirth manote fich zul feinen iibrigen Bäften, und fage te (auf lingrifat): Meifter Slufius hat fid) ged 
fanitten. Die ganje Befellidhaft erfyob ein laus tes Beläd)ter; benn es war Zlllen febr woht befannt, wie emfig er bereitz bie Sdroämme im 2 Baloe unterfuct batte. Ilno hier bey ber Tafel maren bow) fie feine Mieifer! 
$46^{\circ}$

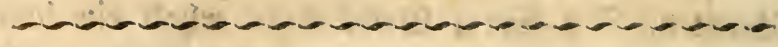

IV. Der Sallimấn (Agaricus, Lepiota, Polymyces. P.)

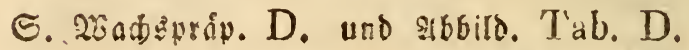

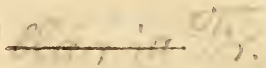

SIS

S- Sie biefer Sthamm in anbern beutffien Säns bern genanit werde, fate ith surdalis nidit in Erfahrung bringen Fönnen. Er ferint iberţaupe Den Deutiofen Eothrififtellern wenig befannt ju fenn. Rin uns miro er jumeilen auth wobl Gtocfs

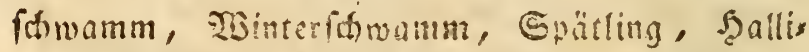
manich und Seckenithwam gefeifien.

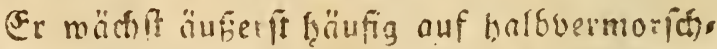
ten Şurzelitbefen won geföllten SHucten, Muften u. Ogl., wie auth auf ber (Frbe im Slober jon

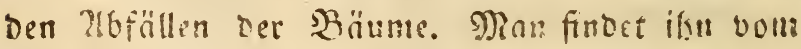
Ende ves Zuguifmonatbe an bis in oen Mosem. ber, ja ten aelinoer Ditrerung oft noch biel fpä:

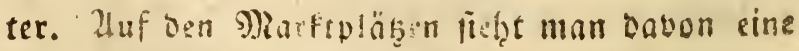
oft gans unglaubliche Dienge. 


Xuf $\mathfrak{B}$ aumftämmen roäch ft biefer S(f)wamm viel lieber als in ber Eroe, uno feine Sgaufen fint viel frïter, zu 6: und Dariber. Er beweis

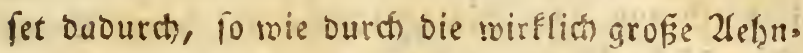
lich Eeit, feine nabe-Berwanotfict)aft mit Dem Stocfichramme (Agaricus, Lepiota, calidicinus Fers.), mefruagen ifgn senn auch ber engुs rifde sncolog Gororty Aguricus stipitis nennet.

Der Şallimafe tot Eeinen TBulft, wohl aber einen jiemfid) ftarken Sirng, ouref Deffen Aubdefnung in oer Sungnd oie camellen verbilllt werben, indem er bett Siano dez f̧uteg mit dim Strunfe verbindet. Diefe f̧aut fețt fich fichtbars lid) bis an ben Srund bee Srunfes fort, Into es frect bemnat) der Gtrunt in berfelben wie ein Jufi in Dem Strumpfe.

Zus oiefer lirfache nennt man biefe Sctwanms art, fo wie alle andere eben fo gebildete arten, eillen Etiefelftumam (Lepiota). Ben oem ges genwärtigen Stiffelfotramme if biefe Saut obs ne Filjlchuppen, uno hat nur einige leichte längs liche fralten, noourch fie fich fotion rosefentlich won bein Stocfichnamme untarfheidet.

Die Scute oer jüngern Indiviouen fino faft Runelruno oder Ropfrörmig: In Der Tolge Greis ten fie fith magrect)t aนร, boch brhalten fie in oer Mitte inner eine frarke, Duncier gefärote 
48

Sabefförmige Erbebung. Der Şut eineb voll

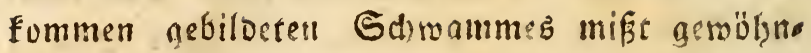
lith 3 Boll im Iur(f)meffer. Er ift geröbntim sunfelbraun ins Tothrgelbliche fpieleno. Spine Oberfiäcte if ctroos feudt) uni baber ein wes nia alänzeno; auch bat fie gevobhnlich viele buns felfärbige zismlid anliegsnoe filsfwuppen, rels we am grabel ferum viel fleiner und viel ge"

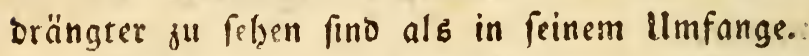

Die \{amellen fino weifi, uno haben eine fefir unrein rofenfarbene Epielung. Im alter werben fie gुelolid. Sie find von mäf̈iger Brei te in zwen, und brenfadien Reiben, uno laufen etwas am StrunE herab.

Der volle fleifhige Strunt ift ben 4 3oll ho(h), und wirb am Brunde etraz bicfer.

Der Sallimaid ändert in ber Srößje von 2 bis z" 5 3oll Der sëbe. Sein Sut fält zuweis len inz 2lfogrnue ober wohl num ine Driven. grüne, zureilen ift bie blafigelbbraune farbe mit einem röţ̧licten Sitimmer burdmengt. Der feuctite Slons iff bald fehr lebhaft, talo Eaum zu bemetfen. Die frilzchuppen fareinen aud zus weilen gans zu fehten. Der Rabel ift öfters feţ: niebergeorücft unb Eaum ju bemerÉen.

Der Sallimafd if angenebm zu verfpeifen. Sein Siefhmack hat einige Zehnlichteit mit dem Freild)e von sämmern. Seruch ift Eaum einer 
an ifne zu comerfer. Man Eutht ifne entroder als Bufaz ju gediinfterem Fleifhe, ober befons bers in Fieift)brïbe, mir einiger Zugabe von Mef̧!, Butter, Salgne. Zur 26 urze pflegt man Sarbellen, Jyfeffer und Zroiebeln ju zeb:auchen. हez ift Der robblfeilfie und am renigften gefährlidue Marftfotwamm. 
V. Der Etodfatwamm (Agaricus,

Lepiota, candicinus. Pers.)

5. Wad)รprạ. E, und stbbits. Tab, E.

รั)

Sil meiner Paturgefdichte ser Defretreichifden Sthrämme habe id unter $\mathrm{No}, \mathrm{X} / \mathrm{V}$. cine be= fonbere 2lbart bes Stocfifhrommes geliefert. Sier folgt er in feiner geroölgnlicten Biloung. Sch benilse zugleid dicke Belegenbeit, einige Febler zu berictitigen, bie mir Damable ent. wifdt find. Sich bielt nährmlitit zu iener Beit now ben sallimaid für eine blope labart bes Stocfichrammes, umb ruffe nicht, ba官 ber Ag. Polymyces Pers, unfer gemöhnnlider Sal. limaft fen. Siernuz flok bie unrid)tige 2lngabe Huber bie erftaunliche Menge bes zu Markte ge. brachten Gtodfichmammez. EFz ift näthmlich hie. von bie ganje Summe des Sallimajh abjuzie: 


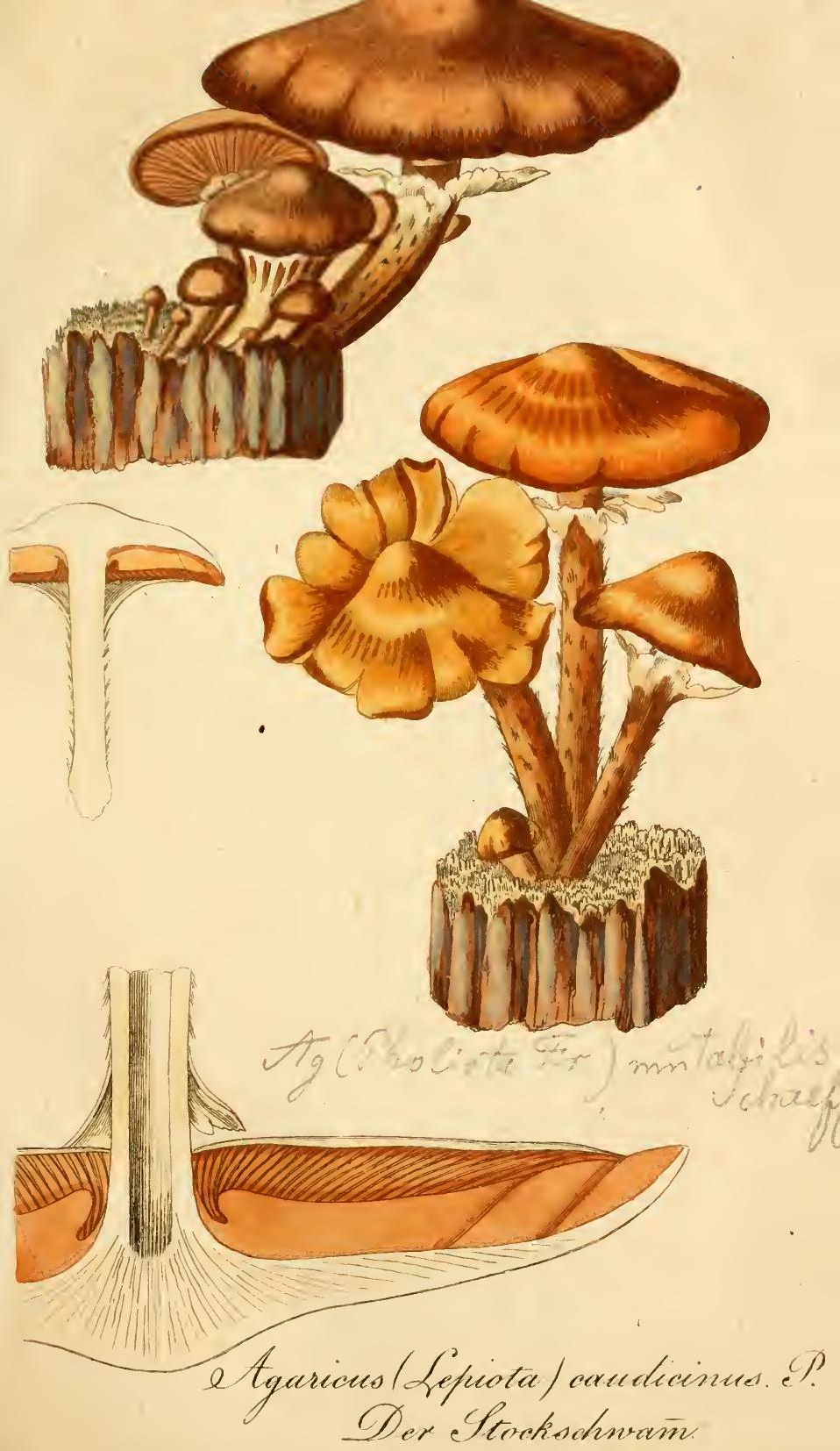



hen. Ifuch ift ju bemerfen, baßz man b̧ier nod) manthe andere Strommart!n, f. B. Den (aud)= fotramm: (A. Gymnopus alliatus P.), ben Fresling (A. Mycena, esculentus P.), ben Drehting (A. Pleuropus, ostreatus P.) uno noch meţr andere mit biefem Rahmen bejeidnet.

Unterbeffen ift es boh wahr, bas in man, then Jabren voll bem mirflicten Stocfifiwamm eine grobe Menge zu Markte gebractit wiro. Ifuch trifft man ihn oft untermengt mit oem

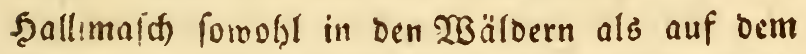
Diarte all, und formobl bie 3eit als der Se. brauch und bie Figenfitaften find unter benden ubereinftimmend.

Man zrennet Den Stodfidronam aus bem genabelten, meiffens bellbraunen, int 2llter am Rande etwas aubsefómeiften glatten und etwas feuttiten Şute, aus ben blaßz Zimmetbraunen, am Srunde ausgefdnittenen uno am Strunke etwas herablaufenden samellen, aus bem meis dien verweesliden Singe und aus bem ziemlid bünnen unterf̧alb iftwärjlichen Strunfe, beffen lleberzug fidf) auf und auf in locfere faferigte Sd)uppen serflücet.

Der in ber Jugerio volle maljenfürmige StrunE pflegt im 2llter boht zu werben. Der haut ift nieorig; zumeilen ficlägt er fith) aud) jurüct in bie 


\section{2}

Seftalt eincs Trichterb, ober jerplngęt am Ranbe f̧erum in häufige S.thliz̧en und riffe.

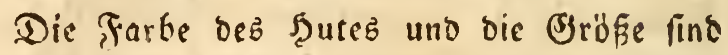
febre berïnderlich. Iluch Darin ift er Felgr unbes ftändig, vafs er, nath Zerbältnif́s feines Stan. bes, fich bald fhief balo gerabe aufriditet.

$E_{i}$ wäctift immer an moderndenl Solje, an - orgefforbenen sgaummurzeln u. ogl. Einzeln Eummt er äuberft feltell vor. 2lber fo grope şaus fen wie ber f̧allimafd bilbet er bod) niemahlz. Zluf ber bier bengefüuten Rupfertafel fin's zwen von bell gerwölinlichen Formen biefer Sd)wammart vorgeftellet worben. 


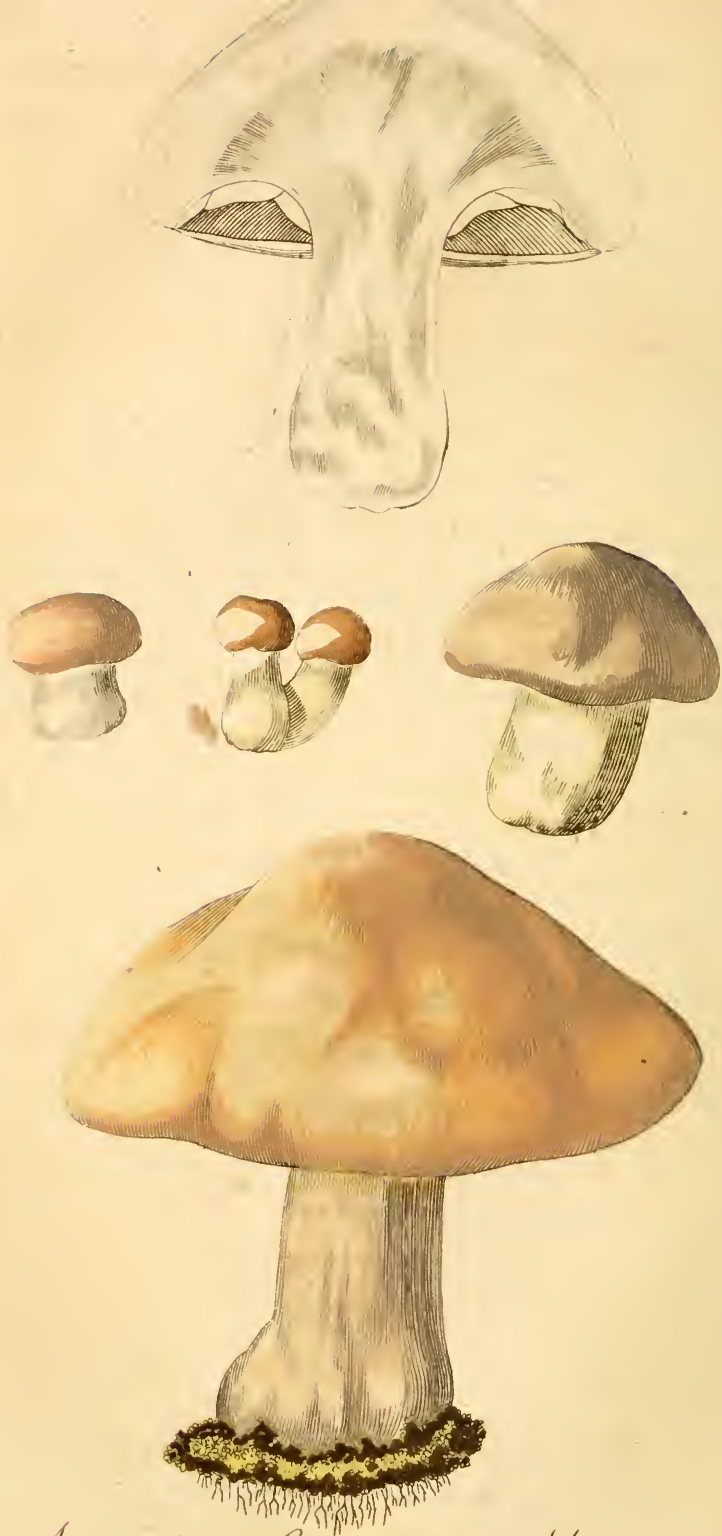

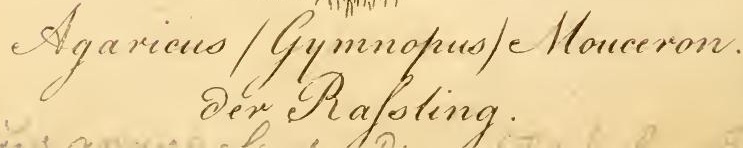




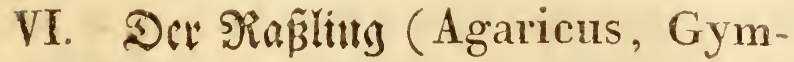
nopus, Mouceron.) ·

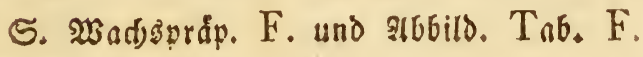

Ser Rafling, Niösling ober Moucerun ge.

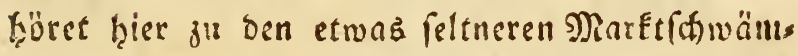
men. Man mús aber wohl merfen, dás biefe fämuntlidte Benennungen verfdfievenen andern effbaren Sthwämmen ertheilt ju werben pfigen. 3. $\mathfrak{B}$. Der కaudfinwamm wiro audf fo getheis fen. Dennow glaubse ith hier den Dialsmen פiaflling benbetgalten zu müffen, theilz weil er einsig unter bemfelben ouf unfern Märften bes Eannt ifi, theils auth, weil ich feine Naturges

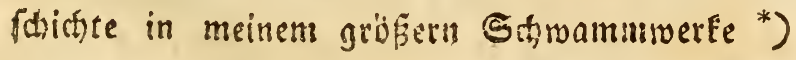

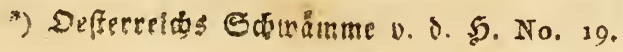




\section{4}

unter eben bemislben abgehandelt habe. Job werbe in ber Folge bie andern gleidnabmigen S.tmämme burch) andere feftgeleace Benennun. gen unterfoneiden.

Der ädte Rafling it ein Sdrramm obue Bulf unb ofine Ring. Bon Farbe ilt ex uns

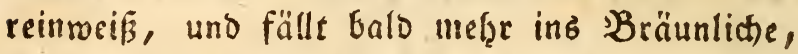
balo ins Braue. Er erreicht felten über $2 \frac{1}{2}$ 3oll an Şähe, uno fein om meiften auzgebreiteter Scut hat benläufig 2 Boll im Durdmeffer. Sos woḩt ber Şut als ber StrunE find nuß̧erordent, lich fleifhig, berb uno yoll. Die Samellen fino weiślich, überaus georängt, feḩr fómal uno

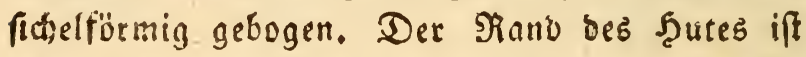
immer lef̧r beträ(t)tict) eingerollt.

Defters wadhen ifgrex mef̧rere vom lll. fprung an zufammen, allein gewöhnlitid lebt ex einfom.

Man fammelt bell Rafling zur Zeit ber Epifmordjel, D. i. im Nan uno Sunius, in Eebirgbraloungen, zrifáen Bucten und Ei, wen, allroo er aus ber Eroe aub Dem Nober ber Ifbfälle uno an ben verborbenen $\mathfrak{B}$ urgeln Der Bäume emporblüf̨t.

Err iff, befonders im jünseen Ilter, über=

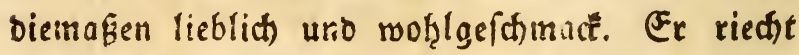
zwar nicht fo heftig, aber, wie es mich beudt), robl not) angenebmer als bie Trüffel. Man 
förnte feinen (Seruá) faft mit vem Brerube Der Iurifeln veryleichen, menn nicht immerhin ber gemiff: Schwammgeruch tengemifht wäre, wel. hen man jetod) aud an allen andern ef̧baren 2irten erfenutet.

ग) mäblt für bie Sïthe nur die jïngern Stücfe, und pflegt fie auch jum fpätern Bes brauthe getrodinet aufzuberobren. Zu biefell Ens be pfiegt man fie an einem Broirnsfaben sios. ternolferartig anzuiafien, und in oer freven suft nn einem trocfenen Drte aufjubiängen.

Derfoona Agaricus Gymnopus gravenleus fteint nicht bieber ju gef̧ören, meil er bie Dicfe bes fleifter bom Sute nur auf 4 linien angibt, auch hätte or bann gewif́ des Sowerby reinen Ag. graveolens anfübren müffen, wels wer alleroinge oer nälsmliche Raßjling iff.

Jd) soüfte zmar Eein Bevfpiel, vafi jemano

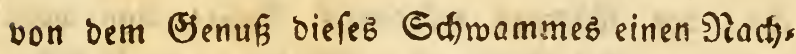
theil an reiner Seruntheit exfahren hätte. In. beffen fareibt ooch Janus गुancus an feillen Freuno ßBartarra im Sabre 1744:, Burreilen

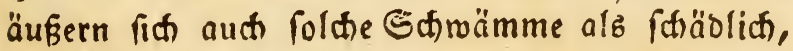
meldie man fonft allgemein für bie beften und fir bie unfhäblichiten zu balten gerootant ift, wies. B. diejeninen, die mir Prunuli ober Pruneoli (ans fer Raklinq) nennen, und romit die Tafeln bes

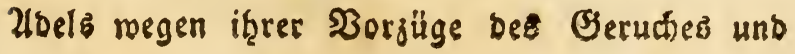




\section{6}

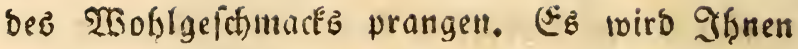
wohl noh befannt fenn, wie vormable einige un, ferer anfebulidfiten Mitbürger fict Durch ben Bes.

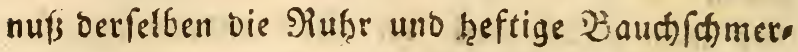
zen zugezogen baben."

It) erfläre mir meinerfeits biefe und anoere äfonlidte Erfoteinungen blof baburd, baf man auch) von bem gefundeften Schromme vergiftet werben fönne und müffe, wenn er zu alt, faulig oder von vielen Maden berwohnt ift. Dief́f fann nun bey fehr feudter uno marmer 2 Sitterung viel leidter als ein anberes Malll geidhehen. 2lud Ë̈nnen vielleid)t gemiffe beenmifhungen gefdehten von äbrnichen fäblicten Scträmmen, und elro= lich ift Doch Der Mienfich felbit nicht immer gleich

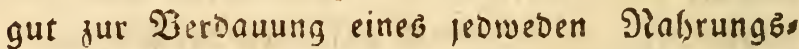
inittele vorbereitet. Whie viele Taufende find nicht fation von dem (Senuf ber Eroäpfel, ber झुfirfis he, oer Rirlchen erfranft? Sollte man wobl Defimegen die Eroäpfel, Pyfirfiche uno Ririchen für giftig oder für verdäđtig erélären? - - 


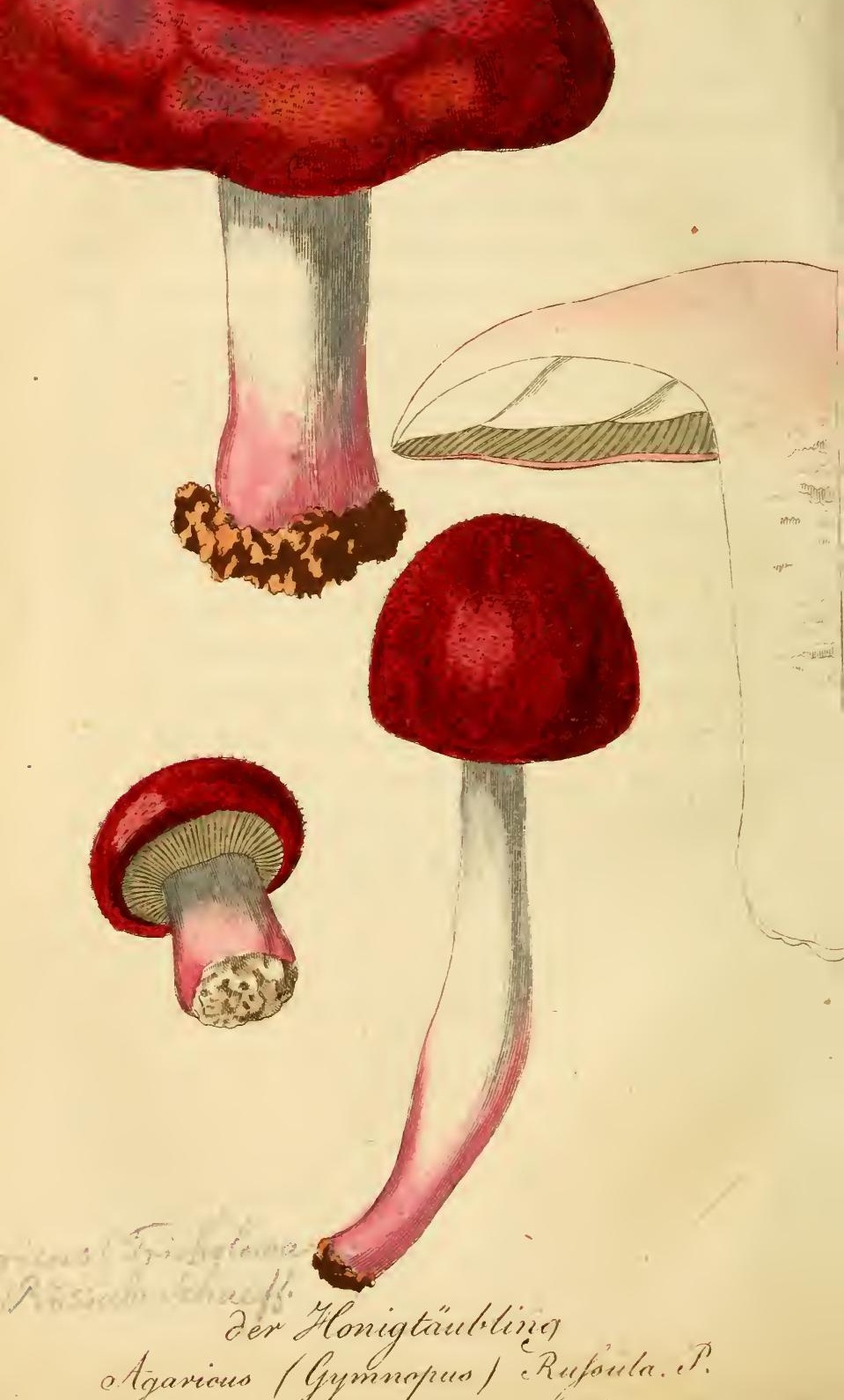

Q

Der Ylonigtruletiner

otgarious (Gymnopus) ¿Rufoulr. T. 


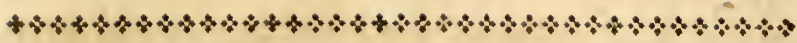

VII. Der Şonigtåubling. (Agaricus Gymnopus Russula Pers.)

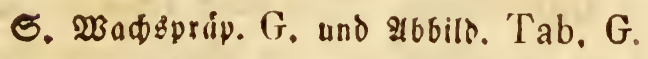

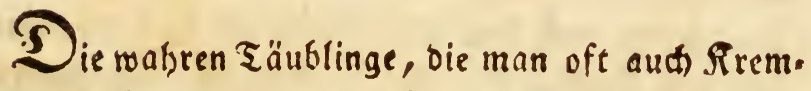
linge Brünlinge ober Frauentäublinge nennet, follten nach meinem Urthęle gänslid von ben Märften betbannt werben, da au由l die beften uns

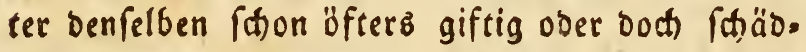

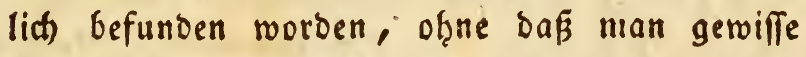
Rennzeidien angeben fünnte, aus weldhen fie fï

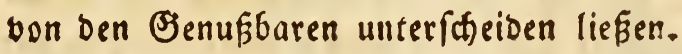

Jeerfoon hat frenlid wobl unter berienigen ZGtḩeilung ber Blätterf́twämme (Agaricus) bie er Täublinge (Russula) nannte, eine $2 \mathfrak{r} \mathfrak{r}$ d)aracterifirt, welder er ben BBonnaţmen Des ¿̨̧aren (Agaricus Russula esculentus) zu ero

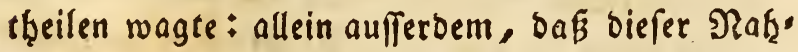
me nifit beftef̧en Eann, weil swen verifiedene 


\section{8}

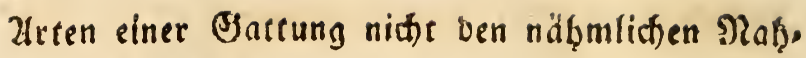

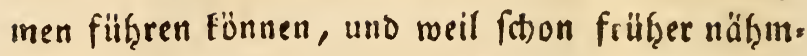
lidh unter ber 2lbtheilung von Mycena eine an. Dere 2frt $A$ garicus esculentus genannt wurbe; to ift auch sie bikherige Beftimmung ber 2 titen yon $S$ wrämmen uno inbbefondere von ß̊ätter. (đ)ıämmen zu ungervis, als daßs man bas lebes urro bie Befundheit ver Menfhen barüber aufs Spiel feg̨en fönnte. Man fann einen Sdmamm nur bann für gefund und bes allgemeinen Bebrau. des fähig erflären, wenn veriährter Bebrauch und fanbhafte MerEmale feine llnifhuld beweis

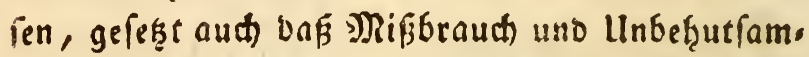
teit bey reinem Eenufe ie Schaben angeridtet bätten; wenn o. E. verborbene Stïcle genoffen wurben! allein bie ädten ₹äublinge find fo wans beltar in ber Farbe, daßz es woht möglich) wäre, DaE bie von Perfoon angenommenen Alten in ber Folgeseit vielleiđte nur fïr Spielarten geljalten werben bürfen, und dafi eben fo wie ihre äuser. liabe $\mathfrak{B}$ effalt auch iţre innern Eigenfhaften ver. haiebenen Mobififazionen unterliegen, bie balo in ber Intresseit, bald in ber 2 Bitterung uno in

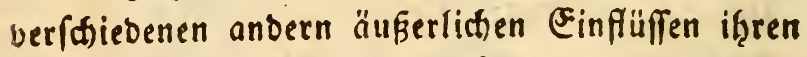
Brund baben. Und auf biefe 2frt mag man fid Die zab̨lreiden Wiberfprïde erflären, bie unв allenthalben in bes feel. Rrapf $\mathfrak{T a t u r g e ( d ) i d i t e ~}$ ber Täublinge auffioken, unb bie une fhon allein 


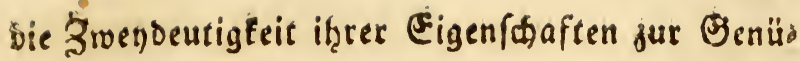
ge erflïren.

Der Şonigtäubling ift fein ₹äubling (Russula) fondern ein Raktfuß̧ß (Gymnopus) uno

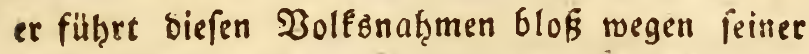
Zehnlidbeit mit ben Säublingen. Ẻr hat einen Heifhigen, vollen, meiffens glcichoicfen Strunk,

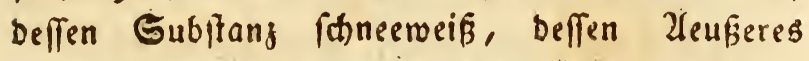

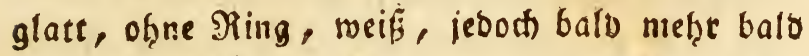
weniger ins Pofenrothe, ober woht auch in $R u$. pferfärbige gewendet ift. Es giebt aud berfhies Delle 2threichungen, fo, Daß ber Strunt baís unten balo in ber Mitte bicker ift, aud) fein $\mathfrak{B e r}$.

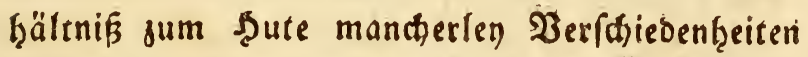
onrftellet, oa er jumeilen Eaum sie Dicke eines Sd)wanentieles übertriftt, Obfhon er gewöhn: lid) an $3 \int 4$ eines Zolles im Durdmeffer, und ete twas über 2 Zoll in ber Şöhte ju erreithen pfled get. Obsn erweitert er fid, obne merflictien 216.

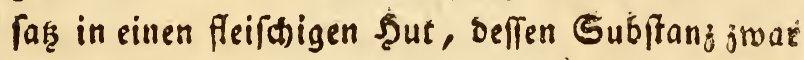

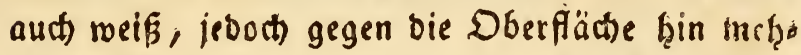
rentheils mit einem rötblichen gaucte gefärbs ift. Diefer sut ift im jüngern Zllter faft Eugelo förmig und am Ranbe herum febre ftark eingerollt. शa(t) und nad) ermeitert ex fid in bie form eines Shirmbahes mit einem Nabel in Bar s)itte, uno ver/dicoenen unrègelniäffigen tiefungen. Seine Farbe if Eupferroth, jeood 
60

gethe fre nidhe felten in eill felye ftarkes Rofenrots uber. Im Alter Der Berwefung wirb fie fhmubsig unb fällt balo mel̨r ins \$̧läulidie balo in Braune. TBas aber bas vorjüglidffe Rennjeicten biefer Shmammart aubmachet, ift ber feine fhuppen, förmige Eries an ber ganjen Oberfäthe bes Şus tes, welcher non ber Dberhaut beffelben herf̈̈mmt, bie fid 2lnfangs in Eleine 20 ärsaten ertiebt, nact. her aber in fothppenartige ungeffaltete Spisen zerplaşt, bie iḩree Feinheit und Nenge megen bent ganzen Scute ein gewiffes ratahes Infehen verfhaffen, weldtes jebo(t) in manden Inbivibuen viel weniger auffällt, uno überhaupt fo wie bie

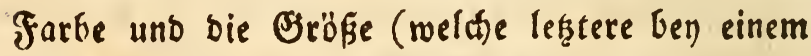
ganj errwadfenen, im Durdimeffer 2 bis 3 Bolle beträgt) verfatebenen Modifíationen unterwor fen ift.

Die Blätter (Samellen) auf ber Unterfeite bes şutes find weiß̧, jiemlid breit, son unglei, der \{änge, nid)t gefpalten, wie ben ben äd)ten ₹ั̆ublingen.

Der Schwamm if mohlfhmedfend, faft wie ein Shampignon. Man bereitet ihn auf mander. Tey) Zrten wie anbere Sd)rämme, insbefonbere pflegen bie \{andleute ihn flein zu jerfdnneiben, und mit Meht und awiebeln nebit Sals uno Mfef. fer in Butter oter Sd)mals ju röfter. Man muß vorfititig alle jene äthten Täublinge auछfonbern, 
bie gefpaltene Blätter baten, aud ift es nüblich bie Strünte der etwas erwadflenen ber länge nad) ourd)ju(d)neibell, und wann fid bie geringfte Spur voll Ungejiefer barin maf̧rneţmen läfit, folde lieber gänzlid wegjuwerfen.

Der Sonigtäubling, ben man aud) unäđten rautenen säubling nennet, findet lich jerftreut in

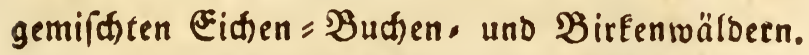
Man bringt ifin vom Infang bes Julius bis in den September zu Markte. 


\section{Dev Raudfofmamm. (Agaricus, Gymnopus, alliatus Pers.)

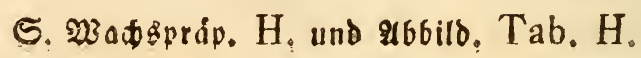

$\mathfrak{N}$ an nennet biefen Éleinen eßzbaren Shroamm auch Rnoblautforwamm, Rafiling, Biefen. forwamm, Mugeron und finder inn in gans Deutfhland, in mahrfateinlid auth not auffer Deutfaland und befonders vom 46. Brab ber nörol. Breite bis jum 50. Ere iff im Frubling und zrar im Ipril am bäufigften, jeood pfiegt er auch im Detober und Povember gefammelé ju weroen. In naffen uno Eüblen Jafiren wiro es jureilen aud) mitten in ben Sonmermonathen gefunben. Er mähif am liebften im Seioclano, o. 1. in feiner fandwarzer Moorerbe, bie aus ver wefenen 26 fällen von fö̆hren, Tannen, Seiben u. o. gl. entifanden if, too bäufiges SRoos bie fotnelle Auetrodinung bes Bobens verţinbert. Ex 

liebt ben Sthatten uno befonders die Brenjen ber $\mathfrak{W a ̈ l o e r ~ a n ~ b e r ~ \Re o r o f e i t e . ~ Z u r e i l e n ~ E ̈ ̈ m m t ~}$ ex aud in Sुrazgärten uno auf WBiefen vor.

STan erfennet ben [auchforroninm aus bem jarten, aber bennow etras fteifen glntten rotls. bräunlidien uno unterbalo faroärgliden, inmen, big hohlen, etwae flachgeorücten uno faft burd). fheinznben StrunE, roelder fich ju einem bis 2 1/2 Boll erbebt, aus bem renig nerölbten, nur etwas fleifdigen, am Ranbe fraḩlenförmig gefaltenen, gelb, oder graubräunlict)en im Mittel. puntte ounkelgefärbten Şute, ber genöbrnlich nur 1/2 ober $3 \int 4$ eines 3olles im Durdmeffer hat uno nur in fib̨r feltenen Fällen gröfer miro, uno

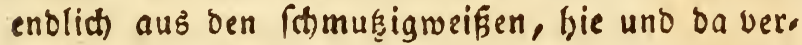
rondfenen mäffig breiten, im Ulter gefrauficen Lamellen, soldte fith leidt unb gan\} vom Sute ablolen und baber einen fef̧r ftarfen Brund an bie Sand geben, iffn eter ber Battung bes Joer. fámammes (Merulius) als oer Dez Blätter, forroanmes (Agaricus) benjujählen.

(E) if ein geroüryfiafter angeneţmer Stivanm: benn obgleid fein Bjeruch etwas ins Rnoblaubartige fällt; fo iff bod biefer fo fef̧r gemilbert, baßz es einen im Siefdmack vielmethe an geviffe antifcorbutifde Rräuter aus ber Fa. milie ber Rreujblumen (Sinnees Tetraonnamiften) erinnert, und graar um fo vielmetis, bo er auth 
64

wirklid etroas gefaljenes mit fich ju führen fafeio net. Man bebient fid feiner nah abgefdülten Blättern jur $\mathfrak{B}$ ürse ber Fleif( brüţen ben ver. fhiedenen Berücten. Man pflegt num ing auf, jufpahren, illoem man iḩn vorb̨er rein abgewas (d)en, une hernat) an ber luft getrocfnet hat. Eben baburch unterfheibet aber ber \{audfforwamm fich ganj befonder von gewiffen Eleinen äḩnlid)en Zrten bes Nifffamammes (Coprinus) ale mel. the fid nitht auftoknen laffen, fonbern entrober ganz in eine fdivarze gaudbe zerflieffen, ober wenigften farwar und faul weroen.

2luch bon bem grofen Nagelfaroamm (Ag. Mycena Alliaceus) deffen (Ëbarke eit rénigftens noc) unbefannt iff, mufi man ben (auch)forwamm unterfdeiben: Denn biefer Nagelforramm ift viel gröber, fteifer uno riecht weit ftärter nad $\Re_{\text {nnob. }}$

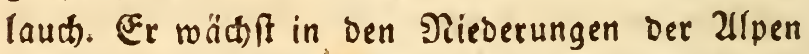
einfaidtig uno fömmt alloort nur in ben Sommer, monathen jum 20 orfhein. 


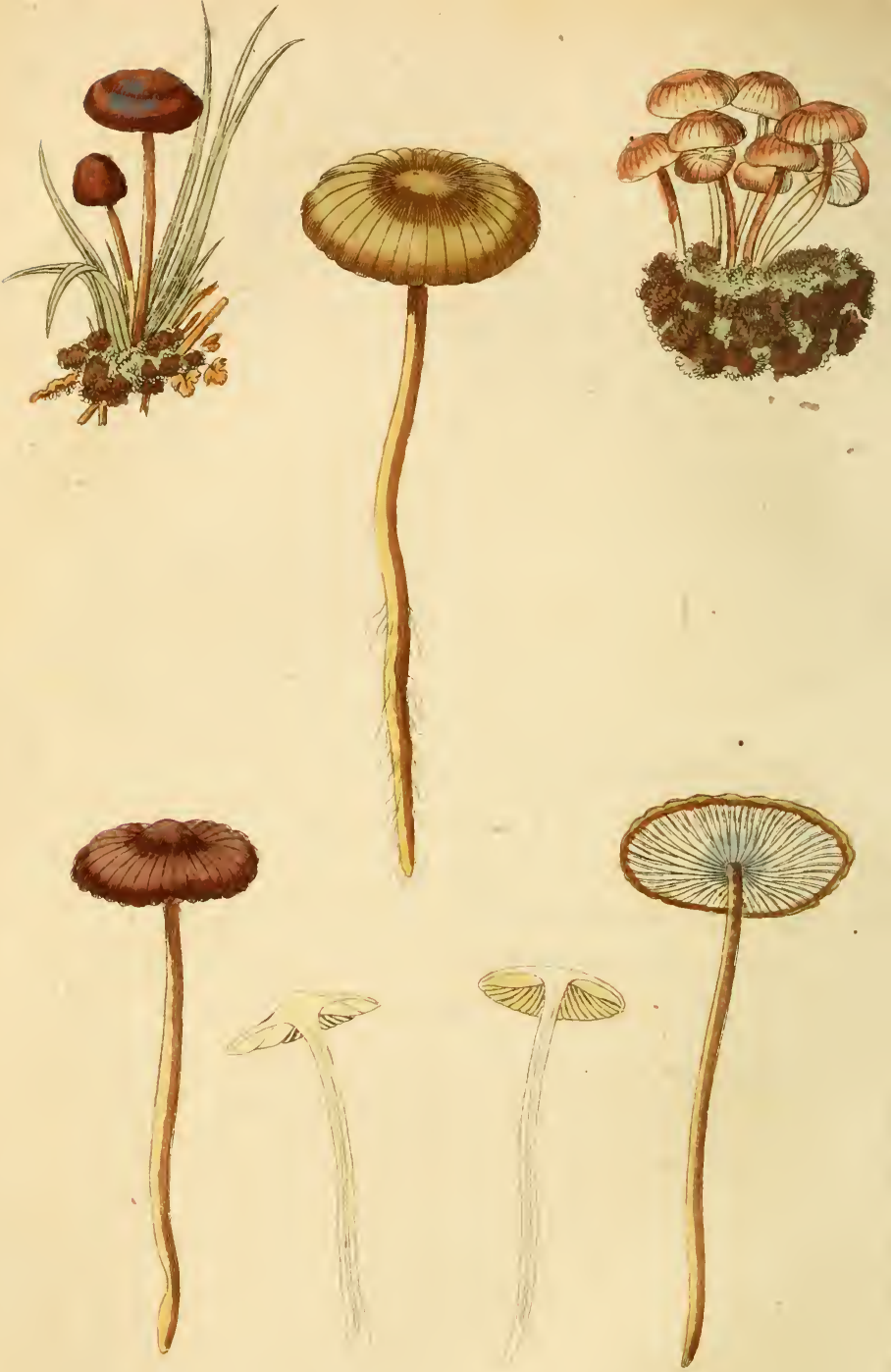

Dere gemeine Naqelochuarman? e Aquricus /ellycena/escutentus P.

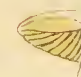

inis 


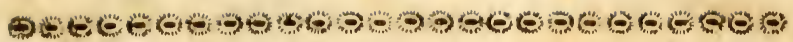

IX. Der gemeine Nagelfhwamm. (Agaricus Mycena esculentus Pers.)

5. 2Baゆspráp. I, und ఇเbbild. Tab. I.

Die ronft now üblidten Benennunger biefer Sđ)wammart find: Siręlling, Rröbling, fleio

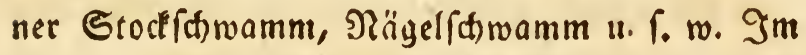
Frans. wiro er Agaric. Clou genaunt, unb Langftede äuffert im allgem. Botan. Reperto. rium I. 3BD. S. 42 bie Bermuthung, daß ber

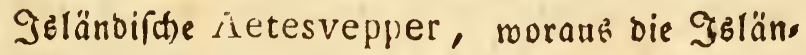
Der eine Epeife bereitell, bie fie Sveppekal nen. tren, ebenfalls bieber gethören möge.

Man findet illen im Trübling und im Serbfit

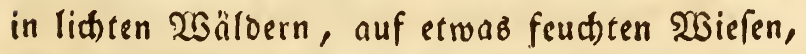
an ben Rändern ber Hecfer und zumeilen felt6ft an ben $2 \mathfrak{B} e g e n$. In Rärnthen bringt man zu Ens

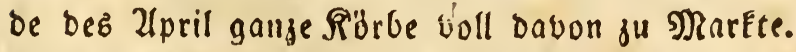

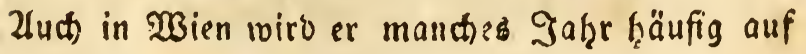




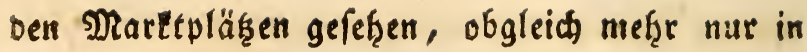
ben Borftäbten.

Es ift eines bon ben minber belicaten $)$ ro. bucten ber Sthammpomona. Man Eaun uno pflege zrar hie uno ba ben Nagelf froamm rof? fuberfpeifen: allein er ift auf biefe $\mathfrak{Z}$ rt elper uns angenefrin als Tecterhaft, beun fein grleifh ift äuß̧erft bünre, und fein S̈efhmact etras widerlid uno bitter.

Unterbeffen nimmt man biefen Sthramin bennod) fo wie ben borigen bur Bermifhung mit Fleiffbräţen uno andern Speifen, roo er in Berbinoung mit allerlen Serwürzen fïr ein gutes ङ̈erübtt geţalten wiro. De guftibus non eft disputanduin!

Er wäd)ft gefellig in lockern şäufden von 3 bis 10 Stücfert, zuneilen aud) einzeln.

Sein hob̧ler Strunk wiro gewöhñlith nur 1 bis $1, \sqrt{2}$ Soll hod, uno bat oft nicht ilber eine halbe sinie in Durchmeffer. Er ift Felgr fart, uno lange nidit to fteif, wie bey bem \{audfffwamme, ift übrigens nach verfofiebenen Ridtungen uno Biegungen aufredit.

Der zarte halbourch/dheinenbe şut hat feldr svenig Fleiff, und ift ber Farbe nadh meiftens jiemlid) helle, bod niemahlen reir weí, fon. bern fällt gemeiniglid ins Selbbraune, Doch gibe es auch Srilce, die ins Siraue, oder ins 
Raftanienbraune fallen. Sat unb Strunt fint immer gleidfárbig, nur ift ber Strunk jebes. maḩl um eill weniges bläffer nle ber scut. $\mathfrak{A n}_{n}$. fangs ift ber scut halbfugelrund, nit ber 3eit breitet er fich aber in ein flactes Servölb aus, Deffen Sitte mit einer Spur von Sabel bejeid, net ift, und eb if now) ilberbieb eine Sathatio rung um benfelben herum, bie faft wie ein 2lugen. ftern auefielst. Zu(t) ber Rano ift mit bunfleren Straf̧lentinien gesiert, bie von ber Einfügung ber Samellen ifren llriprung baben.

Die snmellen find ziemlid breit, lanjett. förmig, etras weitfhid)tig, von Farbe weißflidts, jeooch meiftens fef̧r unrein ins gelblicte ober bräunliche fpieleno.

Zluffer ben bereits angegebenen 216 weidums gen giebt es nod einige anbere 2 lubnalzmen, 3 . B. in ber Sröfe, ba bann einige wobl einen gan. zen Zoll ain şute im Durchmeffer haben. 2lud iff ber StrunE von ungleitier länge, und wirb woht gar über 3 Boll how, uno wäd er vols lenos zroiften faulenden s)oofen hervor; fo fin. Det man feine ganje untere saälfte mebr ober reniger mit einem Barte von äus̄erft feinen Șaug.

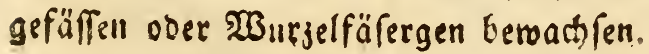


000000000000000000000000000000000

X. Die Ģugemuke. (Agaricus, Pratella, edulis. Pers.)

5. Wađร̊pråp. K, und qbbild. Tab. K.

5 ie Bugemule, roslde man aud weifen Ellegattling, Eb̧egürtel, Şeiderling, Traufalling, Dreitfoling, wie den Egampignon, Rudenmu. dêen, Ungerling, Zlegärtling; Uegerling, B̉rad. büls, Egerling, reeoling, Weioling, WBiefen. pfifferling, 2Biefenfdfwamm, Saioffrwamm,

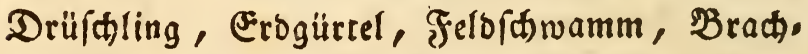
männtben, Şoll. Gewoone Kampernoelje; Brabant. Weyer of Weykampernoelje; Däu. Jordsvamp, Paddehat, Skurvhat; Sanreb. Champignon; Engl. the Toaditool; Franj. l'Agaric comeftible, Campagnoule, Vinous; Ital. Il Pratajuolo, i pradelli, Brife; Span. Agarico campefte; feta, 


xeta, jeta; Perrechicua, Oito; Portug. Agarico dos campos, cogumelo ou tortulho de

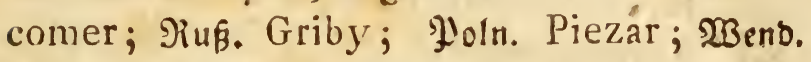
Kuckmack; Ungr. T'seperke Gomba; \{ett. Breedenes zu nennen pflegt, verfiält fich jum ähten Ehampignon, wovon bas nähtiffolgende Sapitel handelt, gerabe fo wie bie $\mathfrak{B a l b e r b b e e r e}$ zur Siartenerbbeere, ober wie im Thierreiche bie milde Raţe zur zahmen. (E) gibt näh̨mlit) aller, len Merfmalsle ber Biloung, moburd) fich bie Gugemule ziemlid frandhaft von bem Shampig. non unterfficibet, wie ber gewöbrnlid hohle Strunt, die Bläffe der \{amellen uno vornähtm. (id) Das Berţältnif́ ber Dicfe jur \{änge bes Etrunfes, inbem bie Sugemufe immer einen Dünneren länglidten uno unterhalb Enolligen StrunE zu baben pflegt, ba bon' ber Strunf bes Eham. pignon walzenförmig, ja jumeilen woht gar ver. fehrt fegelfërmig, uno überţaupt fehr Eur\}, voll uno feifhig iff. Demunyentitet giebt es doct) in allen biefen Stücken jurveilen 2lusnaḩmen, unto menn audi) biefe nicht wären; fo f̈onnte man fragen; of mobl nint vielmehre bie Berfhiebens beit bes Standortes feloft bie llrfactse an ben Beränberungen ber angegetenen Seftalten fenn bürfte, anfratt, baf man von ber lesteren auf bie Rothwendigkeit eines verfthiebenen Standor.

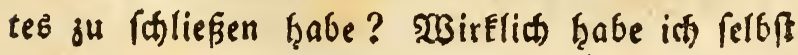




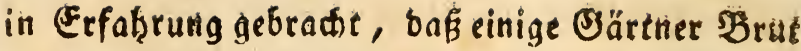
von ber Bugumufe ausnethmen, fie nach 2 lrt Der Ehampignonborut cultiviren, unb wahre

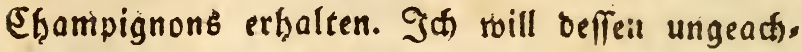
tet hier nidit entfaciden, ob bende nur einerlen oder zrey) verfatiebene 2rten fenn follen. Benug, Daf́ man im gemeinen reben, und bonm (jee braudie felbft Eugemule uno Elgampignon vont sinanber ju unterfheiben pfleget!

Da indeffen-bie Bermandthaft biefer bey, Den Shwammarten fehre grof ift, und bie meiftent Sariftifeller und Floriften foltie nidit bon eino anber unterfotieden haben; to if bie Raturges fhidte ber SSugemufe von jener bes Ehampig. nonb unzertrennbar. WBir fünnen baber nicht mit Servisheit beftimmen, ob biefer ober jener es fey, ben man auch in astoern 20 efttheilen und in Dem neuen Eontinent fo gut wie in bem alten angetroffen babe. Das Meţr des Düngers, bie fanftere Befdaffentreit bes Elima, und bie Ties fe bes Gdattens foleint eine Sauptbebingnif ju renn, weldte bie Bugemufe in ben Ehampignon verwandelt. So viel iftaugemact, baf bie $\mathrm{Su}_{4}$ gemule in iḩrer Öeffalt weit veränderlidier feq, lange nidft bie Siute erreidfe, unb viel öfter ungefund befunden werbe, als ber ähte cultio virte Efgampignou. 
Man finbet fie in gemäfigten Elimaten, im eferen sanbe, wohl auch auf sä̈geln, felten auf Şebirgen, auf Brabangern, im Secibeland, auf Bractälfern und allenthalben ba, ro Düns ger von $D$ ferben mit andern Dünger voer mit Erbe vermifat vergraben liegt, ober wo foldher lange gelegen und wo bie burd)gefinterte Jaudie Den Boben burdorungen. $\mathfrak{I}_{\mathfrak{1}}$ SGärten, auf Exercierplägen, in Sagern, ober feloft in Stäb. tell, wo Yyferdebünger vormahls gelegen, ift Daf̧er if̧re Errfheinung niđts felteneb. Shre Zeit if zroar ziemlich unregelmäffig, Doth Eomo men bie meiffen vom Gulius bis in ben Sepo tember zum 3 orfitein.

Der StrunE diefes Ectwammes ift balo meţr bals meniger aufrecht ober gerabe. $2 \mathrm{~m}$ Srunbe iff er immer etwas allfgetrieben, zumei. len Fehr Enollidit.

Er iff glatt, obgleich) unter bem Bergrö. ferungsglafe feine Dberfähe fehre feinfiljig ere fitheinet. Seine farbe ift weiflich, oder bodt wes nigftene immer um vieles blaffer als bie bes şus teช. Nur bann wenn ber şut auf eine monftiöfe Weife flein, ber Strunk aber fehtr lang ift, nue Dann ift die Farbe dọ Şutes und des Struntes

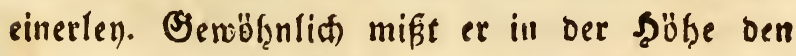
Durdimeffer bes Shutes zreymabl, uno feine Didfe beträgt in Mittel ben 5. ober 6. Theil 
oeffeltert. Zllein es giebt hicuon gerwaltige 2 us. naţmen. Es gilt feḩr mif̧rahfene Strünfe, bie verḩälınifmäffị viel länger, ober bünner, ober ronft verfruppelt, bucfelig ober baurtig find. Inrendig ift ex gewögnlith hohl, boch hab it feloft welthe gelehen, bie es nicht waren.

Der faut, welcter 2lrfange faft gan\} Eus gelrund geftaltet, und ourd eine ziemlith dicfe Saut nit bem Strunfe un und un berbunden

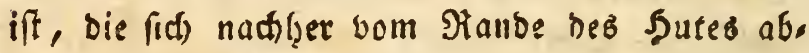
loft, und am Strunfe in Form einer Manfdette fisen bleibt, die man King nennet, ermeitert fich allmäblig, bleibt jeboch immer geroülbt, uno falägt fith niemahl in eine tricterförmige Beftalt jurück. $2 \mathrm{~m}$ Rande herum ift ex imnier feloft ben ben älteften und gröbten Inbivibuen eingerollt. Ex ift felor fleiffig, uno loff fich leidt queer über vom Strunte ab. Sein Fleifd ift feţe

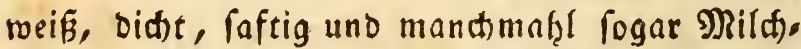
triefend, Seine geroühnnliche Errößze ift ein Durd: meffer bon 2 Bollen, Doit hatte id einft ein Exemplar in meinen şänden, belien Şut 9 ßoll iii Durchmeffer hatte. Es gieft aud allerlen Ubarten uno s) Jiß̈geffalten mit buckeligten, cono centrif( geringelten uno Regelförmigen Şute. Die merfwüroigfte aber bon allen, bie mir zu (Seficte gefommen, war bie aud) hier in ber 266iloung vorgeftellte Monftrofität mit einem 
5 3oll langen, 12 3oll bictin Strunte, einem Sute, ber nidit einmabl einen ganjen 3oll gab nebfi dem gänjlitien abfoluten Mangel ber Ring. baut. Diefer şut ift nun mit einer Sarut uber.

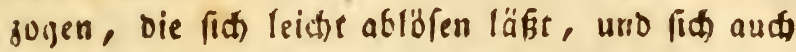
robl felbit jum şbeile abfiälet, renn ber Stronmm feine vollemmene Reife erlangt hat. Die Tarbe biefer saaut, oder fo ju fagen von ber Dberfeite bes sutes ift nad dem Standorte unb ber WBitterung verfitieten. Ie mefre Sorne, Tro.

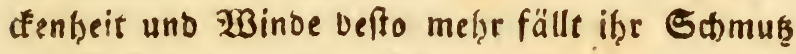
in E Sraubraune. Im Sthatten bingejen unb ben Eubler feudter 2 Bitterung iff fie nidte felten ganz Edincerecis.

Die ferge georängten ramellen finb in bem frühen Ilter, bevor nähmlich oie Ringlinut ser. plaset, fefir blafi fleiftfarben, Dod nie fo Ro. fenfarben wie ten bem äcten Shampignon. Tritt nun aber einmalsl oie Deriode oir Meife ein, wo bie Sthrammerime ausfallen follen, fo merben

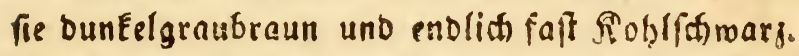
Daben werben fie aud feuct und geben juleste in Faulung uber, nobey fich ein faft unerträglio Wher SEeftane entruictett.

Um $\mathfrak{m e r w e c h s l u n g e n ~ f u ~ v e r m e i s e n , ~ f i u ̈ t e ~}$

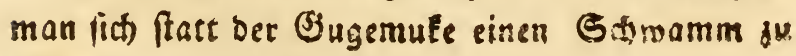
neţmen, 
a) beffen Shut niche weifs, fondern geib, röthlich, violett oder fahefig ift;

b) beffen samellen nidit anfange blafroth fino, und erfi im 2llter fótröriflin wer. Den;

c) Deffen StrunE Eeinen Siing hat, unb an. bers als meifs ober höbtfifens nur ein wes nig befdntust ift;

d) ber audi) nur bie geringfte Epur voll cio ner $\mathfrak{2 u l f t h a u t ~ h a b e n ~ f o l l t e , ~ b i e ~ i n ~ o e r ~}$ Iugend ben ganzen Samamm mit famme

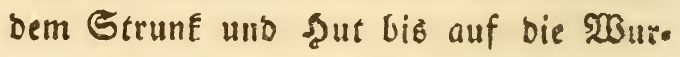
zol verbiullet.

Ilm aber auth bey bem Benuj ber maḩren Eugemufe Feine Orefahre zu laufen; fo rähle man nur foldie Etücfe, beren Şut noth fare gerobibt, beren \{amellen noch röthlich, beren Strunk von Eeinen Maben zerfreffen, beren gleifh uberbnupe noch berb und frifh ift. Man foüle bie Obers haut Des Şutes und den গing ab, verwerfe die Blätter uno foche mit biefen fo zubereiteten Echmämmen eine entimengefpaltene Swiebel oder einen Gilberliffel. TSird jene blau, oder biefer Dunfel fo iff es sin Zeithen giftiger uno föäblicher Eigenfoften. 2uferdem hat man nidis ju tes forgen.

Than pfleat bie unb ba fo!ctie auf obige 2trt fubersitete Erücte von SHugemufen uno Ehampig. 
nons in Elfig ju legen, uno fie auf biefe $21 \mathrm{rt}$ jur

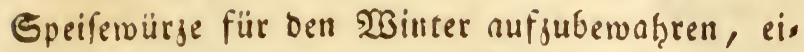
ne Methode bie weit Empfehlungswärbiger und fitherer iff, als bas fo gewüht̨ulidae 2luftroctinen und Dörren ber Sdwämme.

Der Befhmant ber Bsugemufe ift bem bes Ehampignon fo ähnlich, baf idh Davon fo rie von ibrem Esebraucthe bier gar nictits angufübren habe, ba id uIn Wieberḅolungen ful vermeiden meine sefer auf oabjenige verweife, was ith iiber biefe Sachen im nächliffolgenden 2 rrtifel von bem Ehampignon felofit zu fagen gedente. 
X1. Der EFampigron. (Agaricus. Pratella, campestris. Pers.)

ฮ. Madfarip. L. und suttitt. Tab. L.

15

(3) bat biefer Gamamm bie meifen Benennun. gen fowohl im Deutionen alo in ben fremoen Spra. Wen mit Dem vorigen gemein. SRan nennt ihn je. Doch Bo: gugerweife Ebampignon, Serrenich wamm, Sartenf(t)ampignen, Tafeifhwamm u. f. w.

Frenwillig beroormad) fend findet man ifgn in feḩr gemäffigten Elimaten . B. in Stalien, im Eibliden Frantreid, in Epanien u. r. w. Er wirb jebod, obgleid feltner auch in ganj Deutf(h). lano ia wohl in nod) nlebrigeren Sraben ber nörb. liden Breite gefunben. Er wädeft in ber शähe

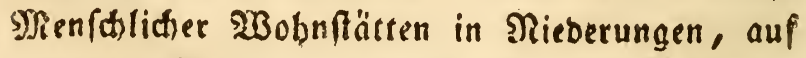
bebauten Stellen, wo die Strenge der Norbrin. 

o: burdf Rerge und Sebalube gebrochen wiro, und jwar befonders auf Yläken roo Pferoebün. ger gelegen, ro die \$ärener und Aferéleute ibre Miffifellen zu baten pflegen, in Deingärten, alteu Epargelbetten, uno nuf गु)tätzen mo offers ') Jerde gereidec werber, jesoct immer nur in etras fautticten Dertern.

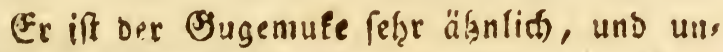
terfacioet fit) von felber $111 \mathrm{r}$ in folgenben Srü. dên :

1) Er if now Fleifdiger, uno frin Fleifh iff nodh famacthafter, faftreidher uno fainer;

2) Tein Gtrunt ift verhältniß̧mäß̧ig Eürjer unb bicker, indem er ben Durthmeffer des Şures in Der fänge nicht übertrifft.

3) Diefer Strunt ift ferner faum meţr als smal fo lang gegen firine Dicfe, auch ift er ent, weder gang Walgenfürmig, ober wohl gar nach untenju abnebmeno, niemabit aber frollig. Er ftebt immer gerade uno hat weber Bicgungen nod Uubrüd)

4) Der Sut iff immer felir geroblbt, olsne शa, bel und mache feine fold)en Mikgeltalten wie die Sugemufe. Bon Farbe if er anfange rein melf, wirb nachlger bräunlia uno feine \$aut ierpflufe 
(it) in fhuppenförmige Schlik̨en. Es gibt 2lbar, ten mić feḩr bunkeln Raftanienbraunen şüten.

5) Die \{amellen find in ber Jugend fetre fäbn, Mofenfarben uno nidte Fleifdfarb wie ber) Der Ëugemufe.

Der Эernectiblung uno Bergiftung halber ift galls bas näbmliche zu beobacten, was id im vorigen Artifel von ber Bjugemufe angegeben trabe.

Der (Serudi) Des Champignon ift zrar nur formath aber both angenebm, uro hat eine dehn. lid) feit mit bem Dufte von 2 baizenmetzl und meifo fen Srofen, obgleich audi immer etrab von bem Srunbgeruat) mit untermenge iff. Der Sjeftimad ift fü̧lich, faft Milchartiz mit einer felyr gelin.

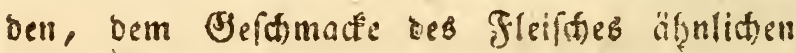
2Bürze.

Man bereitet bie gan wie bie oben befaries bene Bugemufe jugerictete Stilcfe Shampionon

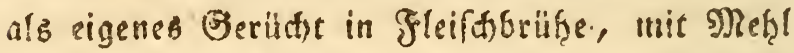
şutter und Mriftrahnt, uno rolirgt fie entweder mit Earbellen ober mit Simonfirialen, פुfeffer

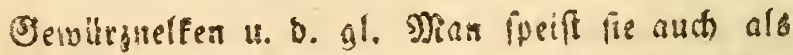
Sallat in Epfig und Baumöbl, aber weit gerwöhn. lidor if Der Bebraud fis nur als augabe ju bers 


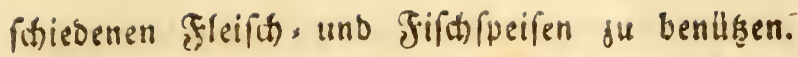
Man giebt fie in bie Suppen zum Eingemadten, unter das Zugemiis in Wafteten, Ragous u. F.w. un Den Befdamact zu werbeffern.

Da biefe Shroümme fehre gefhäţt und theuer bejable werden; fo ift man fthon vor lan. ger Beit barauf verfallen, fie burd) bie אunft zu erjeugen, unb ju vermethren. Die vortheilhafte: fie Methove bicfer Sultur beftelgt im folgenden: Man mact) in einem eigenos baju gewiometen Reller fo viel ba Maum ift, Miftbeeten jurect)t, bie mit Brettern eingefaft marben. Diefe Beeten

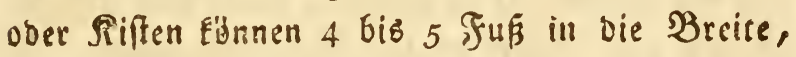

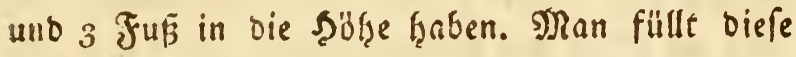
Riften im Spätfommer oder in serbfte mit balbverfaulten, jeood noch nitit ganj ausgetüblo tem $\supseteqq$ ferbemift mit untermengtem Stroh bis an Dell obern Pano, uno giebt von Zsit ju Zeit, wie fid) ber Mift feszet, wieber fo viel nach, daf vie Riften immer ibervoll bleiben. Diefer smift roirb bann nur gan\} wenig jufammengetreten, und nach 14 Tagen ober 3 WBothen, wann bie größs.

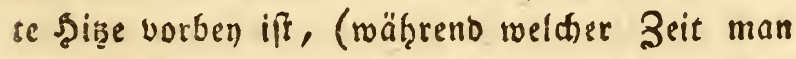
itgn gang menig mit $23 a$ fefer befprif̧et) mit einer 3 ober 4 Zoll bicken Schidte fein geflebter fitwar. jer 刃niftbeeterbe jugebecft. ఇun nimmt man Stü. 
de von ber mohlaufberoabren Ehampigmon ER. be, ffecte fie 1 bis 2 Fub weit von cimander in bie obere Sthid)te ein, begiefit fie ein renig nit ber Braufe, uno bebecte balo barauf bas ganje Beet mit Robrmaten, oder mit Brettert, vie aber nid) aufliegen bïrfen. Gielst man nun nad einigen Tagen, onfis bie Ehanmignons hervor. fommen, fo nimmt man bie Dece weg, unb bes giefit von 3eit gu Beit bas Beet, jeboch nut gans fparfam mit cinem abgefanbenen xaffer, bas mit Salpeter ober Miftjauche vermengt woroen. Die Shampignone welde grof genug fino um verbrauct) ju werden, mußs man borfict. tig mit bem Meffer an Der Eroc abfanciben, um nicht die 5Brut mit heraugjureiffen. Sört nun eir. mal bie \{öfe auf, fo läft man bas Bect trocfen rerben, nimmt bie vergröberte neue Brut heraus unb verwahret fie an einem troctenen Suftigen, gegen Sonnenfliein unb Froft gefdügten Drte guin ferneren Sebrauthe auf.

Znoere bebienen fich wohl aud irgend einer folattigen Stelle im Serwäh) bhaufe und man fant auch im frenen ranbe Ehampignonbceten anlegen, both müfen biele fowohl zur פRachtzeit als wie

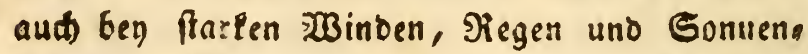
fhein mit Hrettern zugedecte werben, jeood (0, bas bie Bester nid)t auflegen, fonbern immer 


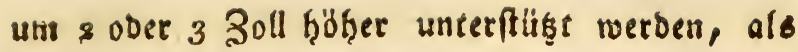
bie fläthe des Beetes fid) erbebt. Die vortheile

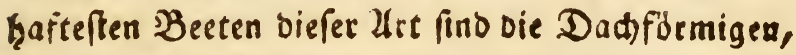
weil fie metse Fläthe haben, uno die überflüfige Feuchtigleit Eefferen ablauf geroinnet. 
XII. Der Reizfer. (Agaricus, Lactifluus, deliciosus. Pers.)

5. \$Bap̉sprip. M, und qubild, Tab, M.

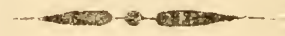

D. bgleich diefer Schwamm nicht reḩr empfoh. len ju merben verbient, fo gelg̈rt er bow ju Den widftiglen, bie hier abgehandelt soerben milffen, weil er an mehreren Drten im Bre. braudie ift, ja fogat in Rärntten, Rernin und Tyrol auf eine äţulidfe 2 rt roie ber Eham= pignon cultivirt wiro, ba er bod mit einiçen frhr giftigen Sthwammarten to viele 2fehnlich.

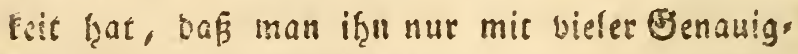
Eeit Davon unterfheiben Eann.

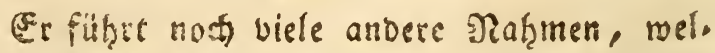

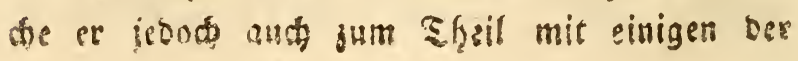



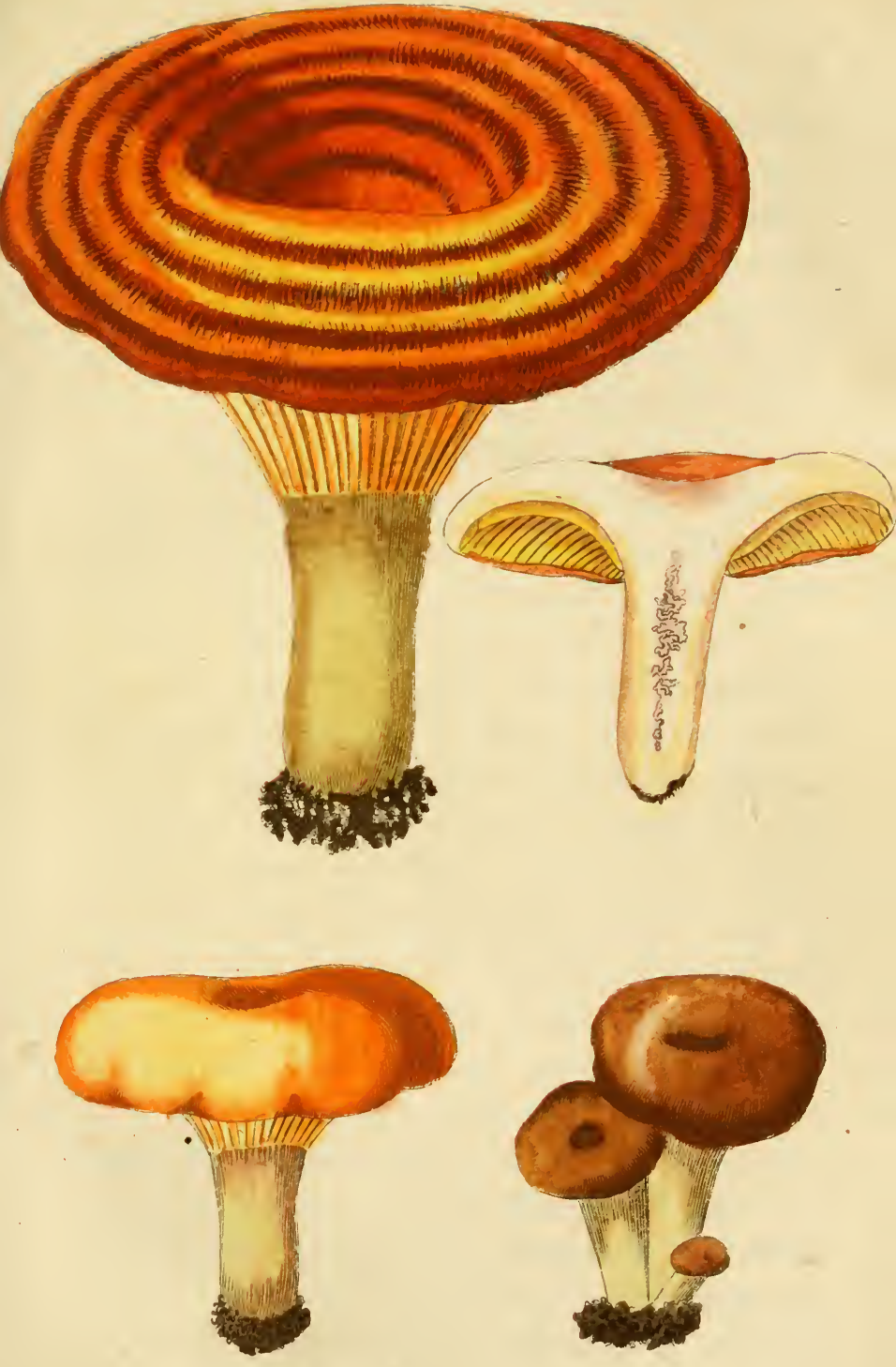

Mer Reither.

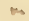

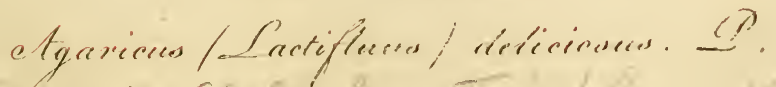



giftigen Ifrten gemein hat. Ee heifft nähmlid bie und ba: der belifate ober lefere Şlätters

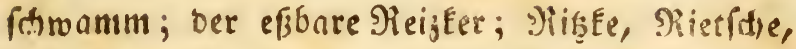
Reiţiter, Salarriezhen; Reifale; Röoling; Rä̋ling; Reißigel ; Ëgesla ; Reibling; Rips pen; Tanneling, Tännling; Förling, Fördf. ling; Şirfoling, Şerbftling; Blutting; Brüt. ling, Brietling, Brüttäubling ; f̧errenidqwamm,

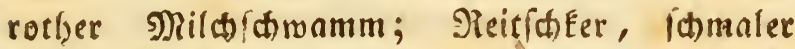
Eleiner Milchftramm; ferner im Şolländ. Lekkere Kampernoelje; Reitscher; Dän. Den lekkere Bladsvamp; eller Riska; Engl. The Orange $\Lambda$ garic; Franz. l'A manite sanguine, L'Agaric delicieux; Gtal. Agarico delizioso; Uovolo, fungo lapacendro; Epan. Agarico delicioso; ’ुortug. Agarico délicioso; Ruf. Ryschik, Royschik; Woln. Ryzik; Bǫ̈̋m. Rizek; Ungar. Rizik; Rrain. Petschenitze; Frinul. Ihmisensiene; \{ett. Sehnes; Efftn. Sened; Siner. Hiàm chuen; Eodhindin. Nam Dec. u. r. w.

Der Reizerer ift, wie man aus ber Mans nigfaltigkeit biefar Benennungen von feloft ges maf̧e wirb, ein wafgrer $2 \mathfrak{B e l t b i t r g e r}$ uno reine

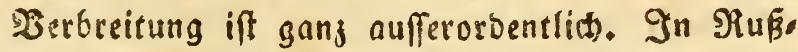
land, wo man überţaupt bie meiften Sthwämo me zu geniefen peflegt, miro or am frärffisen 
$8 \%$

verbrautiot, aufgetrofnet uno logar ju cinter Urt bon Sandelsurtifel verwendet, inoem er in Sals eingematit, oft in ferne Drovinjen be:s fendet wird. Er liebt vorjüglict bectigelegene boe Radelwälber, belonbers Tannennäloer, und fönnt vom 2lugult bis in Den शrovember

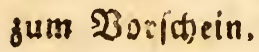

St)on die Römer follen biefen Stwamm

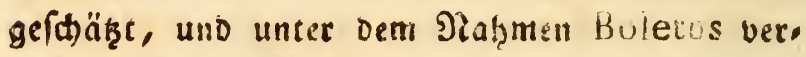
fpeifet haben. Julein ciefe Hehauptung möhte bey genauer llnterfuthung wohl nidt Stano halten. Uno idh glaube bielmeḩe, oaßz der hies ber bejohene Bolecus blof die Amanita caesarea gewefen, von rold)er ficon oben im 3 . Zrtifel ift gehanoelt rorben, uno rooon Plinius L. XXII, cap. 10 fagt: , Inter ea, quae temere manduntur, boletos merito posu. erim, optimi quidem hos cibi, sed immenso exemplo in crimen adductos, veneno Tiberio Claudio principi per hanc occasionem a conjuge Agrippina dato : quo facto illa terris venenum alterum, sibique ante omnes, Ncronem suum dedit.

Volvam enim terra ob hoc prius gignit, ipsum postea in volva, cell in ovo est luteum. Nec eunicae minor gratia in cibo infantis boleti. Rumpitur haec primo nas. 
cente: mox in pediculn corpus absumitur, raroque unquam geminis ex uno pede." Tun iff frolich roht Der פlabme Boletus ben ben alten Loteinern bon ciner fefer auggedefino ten Bebeutung, uno brüct faft eben fo viel aub, als roas wir in Deuticten überhaupe Ourd Daz Woort: Sthamm verftehen: allein Die angegebene Stelle enthä!t eben fo menig eio uen Sruno, moraus man g,ernce fothliểen tönn. te, daf bie Römer den Reizfer gefannt, uno genoffen hätten. Er ift übrigens in Ttalien febr gemein, und roird vorzinglict im Senuefifthen fum Sandel vermender, uno anftatt dafj if̧n die Ruffen in Salj legen, fo roiro er bort in Baumǘl cingemact uno verfenoct.

Sein Sefthmact ift Dem des Bolobrätling jiemlid äbntid). शiur ift er erroas fdärfer, und nod mebr aromatif( D) Defferartig.

Sum : Berfipeifen nimmt man vorzüglich nur junge Etücte, beren Fleifch now berb und olgne sMaden iff. Man fallt Dbertaut uno Blätter ab, setfonneibet iţn in Gtüten, brüht biefe mit fiedenbem raffer $a b$, uno focht ithn nad, ber mit Rutter, Melsl, Salz, uno allerley Serwirisen. 2luch pflegt man inn als Sallat mis Eleinen sieerficitien, Rreblen, Zluifern uno 2 n. tifcorbutif(t)en Rräutern zu berfpeifen. 
Der Pieizer wirb 2 bis 3 30ll ḩoक uno pein Sut bat ungefähr ben näḅmlidien Durch. meffer. Sein Errunt fecti nid)t felten bis jum Anfong Der ramellen in Der Eroe. Sat, \{amellen und Strune fino von gleider Farbe, näbmliç. Dunêl gologelb, bodi fällt er zureilen metrr ins - Biegelrothe. In oer Gugeno ift er etras bläffer ofne Bänoer, und daben etmas feuctit und Eles brig. Im alter geht er inz SBronzfarbene über, befömmt zahlreich jierliche conzentrifale Singe ober gänder über bem scute, bie mit belleren uno suneleren garben abrechfeln, und fpielt bie uno sa, brfonders am Strunt und an ben ramellen ins Orünliab. Die \{amellen uno ber Strunt fino immer um etrons bläffer als ber §ut. Gene, menn fie mit bea Fingern jerrieben werben, pffegen igre Solbgelbe Sarbe in Selbgrün zu verman. beli. Auferocm triefen fie ben jeber Derlę̧ung son siner gelfen Mild), welde nidbt unangenehm

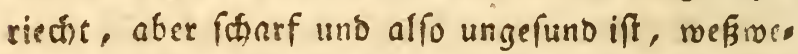
gen man aud bie ramélen jebesmal meglaneidet. Das freifh ift weif unb nur gegen auffen zu ein wenig bon ber allgemeinen farbe deb Schrammes burchorungen. Er Darf nicht fehr reif meroen, fo

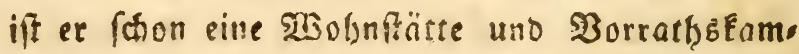
mer ber Maben. Man triffi Dafber felten einen an beffen Gtrunt now boll, und nicht won Snfet.

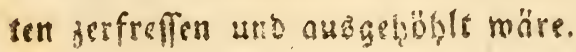


Der Strunf des Reizęers ift Wonlzenfärmig ober berfef̧rt Regelförmig ganz glatt unlo ermeio tert fich nach oben zu allmährlig in die Eubftang Des f̧utes. Die \{amellen find Sichelfürmig fef̧r fomal uno georängt uno wedfeln urregelmäfitg in 2 uno 3 Reilsen von ungleidier \{änge. Der Infange slatte feudite und etwas flebricite, nad). ber aber filzig gebänderte şut if ftion in oer Juts geno im Mittelpunfte vertieft, uno am Ranoe eingerollt, wiro nber juleşt ganj Tridterförmig mit cinem fiarf gerölbten llmfange, an bem man nidte felten allerlen $\mathfrak{3 e r t i e f u n g e n ~ u n d ~ R i f f e ~ b s s ~}$ merfet.

Diefer Shwamm bat einige Ueţntihkeit mit Dein Pïthlinge (Merulius Chantarellus) noch methr aber mit berfdicoenen Zlrten ber Brätlinge oser mildfowämme, mit teran er auth wirflia in einerley Famillie getëret.

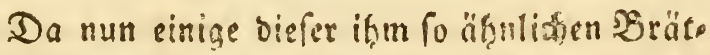

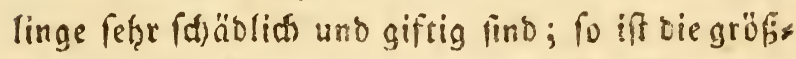
te Beleutfamécit röthig, wern man beum Eins famineln bes Reizfers verhüten will, daß Eeir unächter ober giftiger barunter gerathe.

Man muß́ fich bemnah) Fethr genau an bir fier vorgelegte Befatreibung falten, unb alle jens serwerfen; 
38

a) beren Şauptfarbenidht ein bunfles Fothe gelb, fonbern Srau, Miaun, Meí̈ ober Roth ift;

b) bie eine Sour bon Dorhang, Tulft ober Ringbaut baben;

c) Deren Mild nictit rothgelf, fonbern weif ober bläulich if?

d) relche in Birfen uno nich: in Nabels soäibern rachifen;

e) an meldien Sout, Rlätter uns StrunE methe ale einetei Srunofatbe haben.

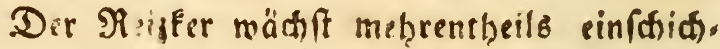
tia; Doch tiff! man ith zumeilen nuch gefellig,

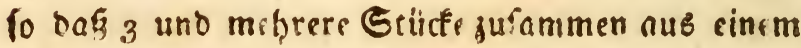
IIninte berbormadifen. Im alter verliert fid fein Fleif( , er mirt trocfen, grünliti uno gegen ben Rand hin faff ourchfichtig. Sein Strunt bes fommt Branoflecten und pin Faferiater 2 Burs selgemebe verbreitet fich aus demfelben in bie an.

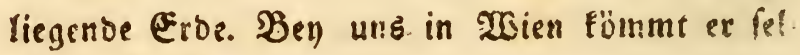
ten o sharte. 


XIII. Der B̧oldopråtling. (Agaricus, Lactifluus, ruber. Pers.)

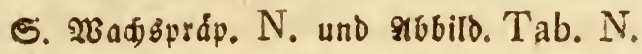

Diefer Bolbbrätling, ben man fonft wobl auct)

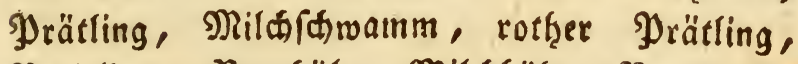

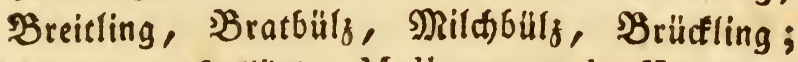
Süßling; Şolläno. Melkgeevende Kampernoelje; Dän. Stegeswampen; Stiwed. Stekswampen; Engl. The milky Agarie; granz. L'Amanit laiteuse, L'Agaric à suc blanc; Jtal. Agarico latticinoso; Span. Agarico que arroja leche; ஒortug. Agarico que tem hum succo branco semelhante ao Leite etc.

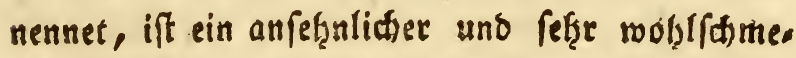
cenber Stiwamm, ber "faft in allen Begenben bes gemäifigten Erogürtels zu \$̧aufe iff, und ben

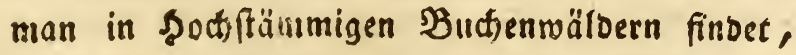
auf maäfingen Sebirgen, wo feudter Boden uns. 
$9^{\circ}$

ţäufigez MHoos feine Decke ift. Man bringt ihn vom halben Iuliub bis in ben Drtober ju Mare. te, oot) gef̧brt er inmer zu ben etwas feltneren uno foftbaren SMarftfdroämmen. E゙r wäh)t gern gefellig uno man finbet ofters ganze Saufen von gröfern und Eleinern burd) einanber.

Man erfennt il̨n ans dem bicken bollen 2 bis 3 3oll langen, uno melir als Bolloicken un ten Meiftens Rnollichten, bon aú̈en glatten ober nur wenig bereiften weiflicton ober blapbräunli. When Strunte, aus ben blajgelben, gebrängten, 59ilatriefenden, am Strunt lecrabiaufenben, ein wenig breiten 3reifigen \{amellen, uno aus bem febr fieifhig gepolfterten rothbrounen oder faft

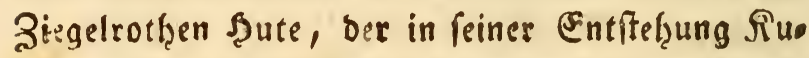
gelruito, im 2llter aber fab̧r ausgebreitet iff, mit eirer ffarfen Bertizfung in oor Mitte uno ver fhiedenen Rrümmungen uno ßuđten im Umfang. Diefer Şut hat gar Éeine Ringbänber, fonbern if einfärbig, stwas bereift uno jureilell meht ober weniger bunfelgeflect. Im Durd)/nnitt hat er ein blaffes etroas bräunliches Fleifa), uno biefes pflegt mitten im StrunE fpäterḩin locker und jellidit zu werben. Der Rano bes Şutes iff feltht im alter noch eingerollt. Sat ein Sctramm nicht alle diefe Merfmable, fo ift ex für teinent äcten Soloprätling zu balten. Uno alle die übri. gen गrätlinge fino entweber verdähtig, oder bact 


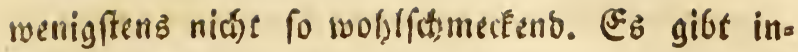
beffen manderley 2lbarten und গaturfpiele. $\mathfrak{B o r}$ allen ift bie Brö̈ze biefes Stwammes fef̨r ver's änderliá, benn er wire zuweilen nod) beträめts

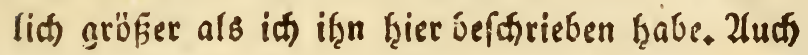
gibt es $D a$, wo viele zufammenmod) (en, allerley Monftrofitäten und Berwadfungen. Sein Oees formack if gelinbe Dfefferartig, fïflid, unb fein OSeruch hat cinige Zlef̧nlict)êt mit ben Dämpfen

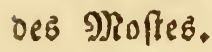

Man pflegt ifnn nad vorheriger 3ubereitung, in Stücfe zu zerfthneiben, in Butter zu röften, mit Sals, Zwoiebeln, Mildrahm uno Deterfilgen zu wïrsen, ober wohl auth in einer Fleifdbrühe, mit etwas Metrl uns gyfeffer zu bünften.

Die Mild oce Goloprätling ift weig̈lich. 
XIV. Der Drebling. (Agaricus Pleuropus ostreatus. Pers).

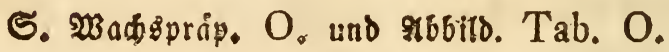

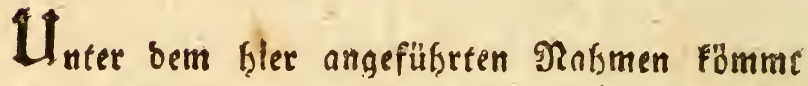
Diefer Stiwamm im Detober und November auf Den Marlt in sien und in Den nafegelegenent Drtf(haften. Ben verfhiebenen Sdififtfellern fübrt er inbeffen aud noch melfreylen anbere Benennungen; 3. B. ber_Zlufferfifwamm, ber 2lufternförmige B̉ätterfकimamm; man nennt if̧n auch wohl yaftetenformamm, in Engl. The oyster agaric ứ. T. w.

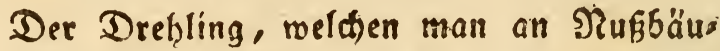
men, Buthen und Fidten, nafie an ber 2 surjel Diefer Bäume in feuditen Begenben finbel, iff ein gefunber, jebod nicht gar befonbers formacts bafter Sthamm, ber viel Fleifh hat und in feiner Gugeno auch fehr faftia iff. Man gebrauds ihn fo wie ben Sallimaj(h, SDilfling u. b. gl. 


2us einer anfebnlichen berben Fleifamaffe erheben fich im färtfiten (S)oränge meţrere, oft etreldie f̧unbert feţr fleifdig gepolfterte, an Bes fralt uno Şrößze äußzerft mannigfaltige, fef̧r Eurs ober gar unmertlids gefrielte, Dachjiegelförmig übereinanber gelegte oberlalb glatte uno erroas feuchte am Şrunbe weiflichte, übrigenz fahle, graubraune ober Raftanienfärbige J̧üte, welche an if̧er Unterfeite mit georängten fotmalen, gan. jen, berablaufenden, am 2lnfang uno am Enoe jugefpişen rosifen samelles bebect finb.

Die şüte fino, wie faton gemeldet rorben, fefcr verftieben, uno diefes Ëommt theils von Dem Druck ber anliegenten einzelnen Säte, theils von ber Etellung ber ganjen Sruppen her. Mand. maf̨l find biele Eruppen nur faum ein paar Boll lang, aflein id habe aud ungef̧euer grofie ges rehen, bie über einen Fuf im Durdmeffer hato ten. Diejenigen Süte, cie bann am meiften eina geflemme find, exhalten oft bie allerfeltfamiten Figuren, fie werben föpfig, Reulen ux Rräu. felförmig, uno find balo jertheilt balo verwati. ren. Da bie Bruppen bes Drehlinges nidt im. mer feitwärtz fonbern öfters aut) borizontal aufs fikgen, fo bemerft man im lestern Falle eine ges wiffe conjentrifote Stellung ber Sä̈te, fo, daßj sie Lamellen alle naw bem s) ittelpuncte feţen, welther gervöhnlith in einer flaffenden Spalte beo 
94

fteht, unb bie zu nädift baber befindliden şüte fino balb auffitenenb balo mit einem, zuweilen fo= gar mit einem centralen Etrunfe verfelsen. Die gröften Şüte fino immer jene, weld)e fid zwifhen Dem Sittel unb bem Ranbe befinsen, fie meffen

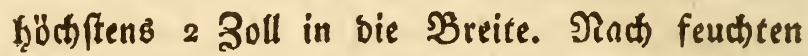
Sommern unb ben fortwähreeno feudfem \$erbfte findet man fie fehre bäufig in alten Dbfigärten und in liđiten 20 nloungen, zumeilen bis zum Ende

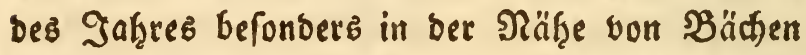
unb in engen Thälern, wo bie $\mathfrak{B}$ inbe wenig 3utritt haben. 

XV. Der Rótbling. (Merulius Chantarellus. Per's.)

5. ※ađşprớp. P. und 2l6bild. Tab. P.

Sa ber Piöthling ju benjenigen Sớtwämmen getgöret, bie am meiffen berbreiter fint und allges mein genoffen werben; fo ift fich nidite zu verwuns Dern, vał er aud fo wie ber Shampignon u. o. gl. Fethr vielerten Benennungen erhalten. Go heigét

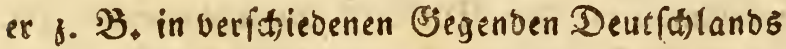
Y) fiffer, YJffferling, Dyfefferling, gelber Gham, pignon, Ehantareille, Pietggeift, Enyergelber Ŗläto terfawamu, Enerfdiwamm, gelber WDefferling,

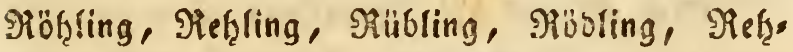

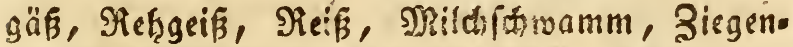

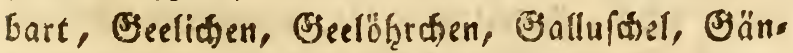
fel, Şimling, Sïnlich. Sodmänoel u. f. w. fers ner im şollänbifâen: Zeemleere Kampernoelje, Chanterelle, greele champiguon, Staa 
$9^{6}$

zenoor, Zaffrankampernoelje, het Merg der Aarde, hemelsch-Manna; im Dänifden Den guule Champignon eller Chantarelle, Scfroeb. Chandarelle; Engl. the yellow agaric or Chantarelle; Słlottlänb. Parddock-stool, Devonfhire: Picksevstool; Fram. Chantarelle jaunâtre, I’ágaric Chanterelie, gerille, Brigoule; Ital. Gallinaccio; నapolit. Galluccio; Gpan. uno Portug. Agarico cantarillo, Rrain. Lesitohe; rett. Gailenes etc. etc.

Der Ribthling findet fich burch gang Europa in Bebirgägegenden vorzügliđ in Fidtenwölibern, jebod) fino nud Sెud)enwälter und Jaibeplätze niche felten fein Stansort. Ex fömmt in manden

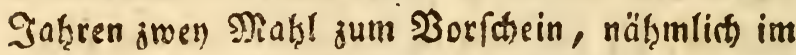
Mai) uno in Epätfommer. Die Siöthlinge bes Frühlings fino etwas Eleiner, Glaffer von Farbe und milber als jene bie in ber froenten a dernde gefammelt uerben. Diefe iff hingegen ergiebiger und benbe dernoten fallen gerabe in bie Monathe, in welthen fid), $b_{81}$ uns jum wenigften, bie

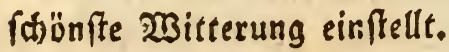

Der Struné bes röthlings ift umgefeţrt Regelförmig $1 / 4$ bis $1 / 2$ 3oll biff, voll, fleio ífig, berb, äuferlich glatt, rüthliçbraun unb bereift. Er ift gewöbnlid fef̧r Furg unb geht balb unmerElich in ben şut über. Diefer ift Dotter. gel6, bod geht er aut balo mef̧r bald weniger 
ins Pethfarbene über unb mande Indiviouen pran, gen vollents mit ber einlabenden Sd, minfe ber

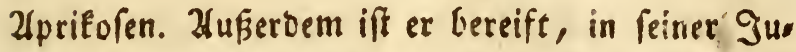
genb Rugelrurt und cingeroilt, Dann auegebrei= tet und flach gebrückt, endlich aber aufgeridtet uno Tridterförmig. Seine Dberfläure iff meis ftens uneben unb befonders am Ravibe wellens förmig ober ausgefकweift. Sein vollez berbes Fleifh ift weis ober fahl. Man Eann diefen şut für nidt)ts weiter als für eine fortfétung uno

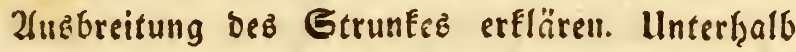
iff er ganz mit gleidfärbigen Lamellenartigen Falten bebecft, weldhe in geträngter Reiţe vom Rande bez f̧utes bnlo feidter bald tiefer in ben StrunE herablaufen unb fin unmerÉbar verliehren. Diefe fins ebenfalls bereift, sertheilen fid ohne alle Dronung und wathen öfters über bie Duere jufammen.

Mall triffe zwar ba, mo ber Rötbling zu şaufe ift, meiffenz eine großje SRenge bavon an, Dennod) rädift er, ber Regel nact), nur einjeln,

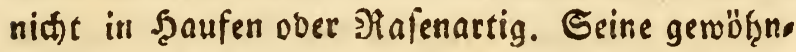
liabe Erö̈ße befteht in einem szölligen Durdmef. fer und einer gleiden Şöh̨e. 2lužnaf̧men gibe es inbeffen auch hier fo wie bey benen Schwämmen überḩaupt. Es gibt Mlonftrofitäten ber Maaje uno ber $\mathfrak{B e r w a d j u n g e n . ~ E b e n ~ f o ~ g i b t ~ e f ~ a u d ~}$ 
Fälle, in welden man iţn it şaufen zufammen. machiens beobahten Eann.

Man plegt diefen Sthwamm nller Drten

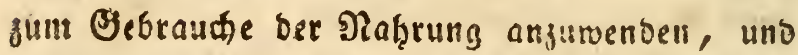
es ift nod) tein Benfpiel befannt, baß je sin Menich burd ifin ober auch nur burd eine Bers wed) slung mit bemfelben wäre vergiftet morden. Iogeţärtete Subjecte (qui temperamento et digestione bœotica gaudent!) verzeţren if̧n rol unb of̧ne alle Borbereitung. 2lllein Den zärts (id)eren Stäbtebewofinern möbte eine foldhe Roft wohl mandife llnannelgmlichét von einer İnoiges fition, Eolide, Erbredien u. b. gl. verurfacten. Man plegt baţer in unfern Rühen bie Oberhaut ume ben ganzen Saltenrocé abzuziefien, bie nach. her zerfonittenen Stücfe mit heißzem waffer ab. subrüfen, unb bann bas ganje Eerüct) mit einer Sugabe von Butter, Saf̨ne, geriebenen $\mathfrak{X a}$ aizen brog u. o. gl. zu oünften. 2fls $20 u ̈ r g e$ ninmt man nack Belieben, Salz, Iyfaffer, Earbellen, Zmies

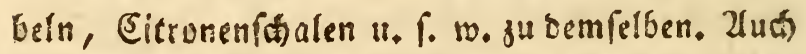
möbien geffoffere Mandeln ben Beffimast davon

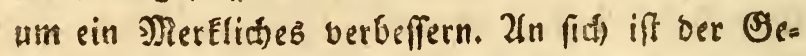

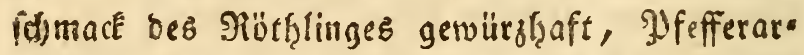
tig, weß̈megen er aud Dfefferling gerannt wirb, sutb fein Geruch erinnert an ein Semengfel bou Begärbtem Leder uno Earamom. 
Ulm biefen Shwamm mit feinem anbern ju verwedfeln, mußs man näd)fit ber Einheit ber Farbe nod) vornähimlich bie STlätte bes Şutes unb bie umgefeetret Éegelförmige Beftalt bes vol. ren StrunEes im Sesädtnifise beţalten. 
XV1. Der אubfirgling. (Boletus sub. tomentosus. L).

5. 23aథspråp. Q, und 26bitl. Tab, Q.

\section{S}

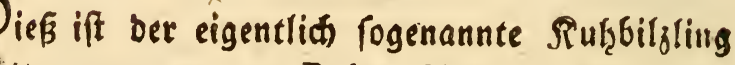
Bolet commun unb Bolet Chrysenthere Des Bulliard. Man nennt if̧n aud wob̨l Biflling allein. Dow finb bie $\mathfrak{B}$ enennungen der $\mathfrak{B} i z_{z}$ linge

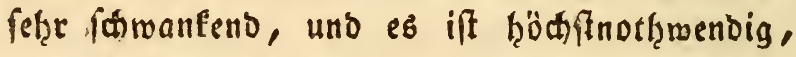
audf im Teutfiten bie Sdimammbenennungen fefto oufegenen, weil burd) jene Unoronung uno Bero

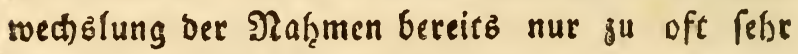
bösartige Zufälle veranlap̧t morben.

Er wädhft aud) bey uns feţe ţäufig und ift

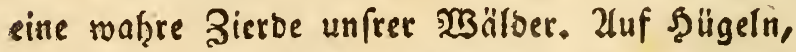
bie mit altent Fiden, Budfen, Lllmen, uno

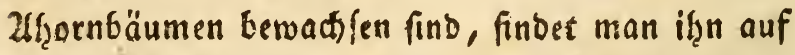
3er Eablen Eroe im Mober ber Banumabfälle, falo einjeln fald gefellig vom Juniue bis in ben 



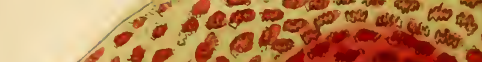

50.008 ioritow ar +1 1. 15.150

(1)

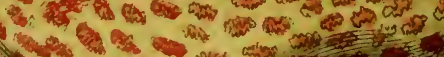

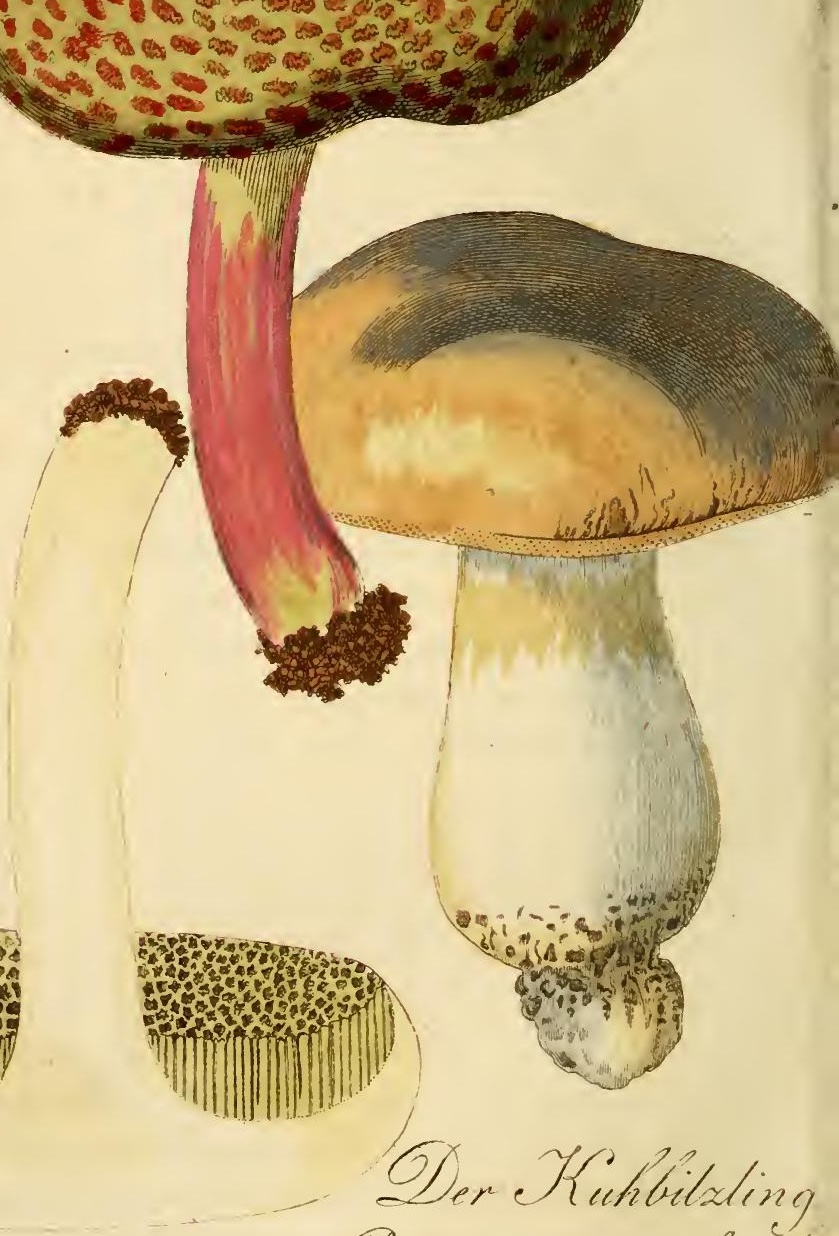

का

$30 y^{2}$ b

Ho d $\mathrm{C}^{3} \mathrm{H}$

- pa +50

$6 \%$ 언

करे कीजा

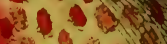

(c) 40 is

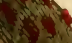

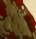

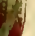


November. Seine jieriide BSeftalt unb feine bun= te Färbung belebt und erf̧eitert bie ernfte Dun. Eelfeit ber Saine, fo wie bie bellen Beftirne die mitternäd)tliche Salle des Şimmels. Man muß fich aber wohl in 2(d)t nef̧men, if̧n mit anbern giftigen 2rten $\mathfrak{B i l}$ linge, bie oft nicht reniget fđ̆ön fino, uno mit ilsm an einer Stelle burch pinander machfen, wie z. B. nit bem פy fefferbils, Blutbilz, Schweinbilz u. o. gl. zu verwech) feln.

Die Rennjeichen Des Rub̨bilslinges beftehen im folgenden :

Der StrunE ift voll, bon aufen meiftens gelblich und im reifen Ulter geroühnlich mit einem Blutrothen 2loergefledte Nejartig bemahlt. Das Sfleifh ift reidffaferig, weif́, im fpätern 2llter gelblich, unb läuft mandimahl, jeooch nur langs fam an ber \{uft grün ober bläulid an, wenn man ben Shivamm in Stücfe zerfaneibet. Der Şut ift fieifdig, gerölbt, uneben, bon Farbe graubraun uno feinfilgig. Niabt felten mildt fith aud), befonbers am Sipfel eine Blutrothe Sins te in feine Srarte. $\mathfrak{B}$ en trocfener 2 Bitterung zer. foringt feine Dberhaut oft in fefor viele fleine Iäppden und ba bann bas nakte Fleild gelblid herbor fieft, fo geminnt ber Şut baburd ein fef̧e artig getäfeltes 2 nfeţen. Seine untere fläde ift mit verwachfenen jiemlid langen R̈bhrchen bes fotst, beren SRünoungen offen fteben, uns eir 
mit SRabeln burdffochenes SRähtetifen vorftellen.

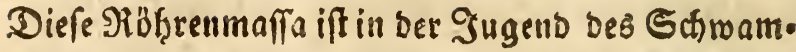
mes überans fein, ungemzin lebhaft Shroefel. geit uno faft gefwilofien. 21m Enbe ringegen wirb fie gelbgrünlic : vie Mänoungen werben beträ๘t Yich, jeooch ferre ungleid) an Seftalt und an Srös Ee. Die meiften find ecfig vom Seitenoruct der anliegenden. Indere ragen weiter hervor und die dem Strunf an näciften fuld, pflegen wie aufo gefchlizt an benreloen ringeum hernb ju raufen, uno fich gans unvermertet in cin b!ofes 20ernes zu verlieren.

(ङ) gibt auperorbentlich vitle Barietäten unb פaturfpiele unter viefer Saimammfpeciez. Jene Der Farbe find bereits bezei⿱nnet worben. Der Fils ift in ber gugend am Deutlidften fu bemers Een. Dow gitit es ant) grofie bie now filzig fino. Die erbeblidffen 26 änderungen erleibet vie Ş: fralt! Der Strunk :ft 子. $\mathfrak{B}$. zuweilen bitf, Enols lig, Eräufelförmig, ober bünn, 2刃alzenförmig, gernice ober auffeigend. Der Sut if Rugeiruno, oder flach georückt, regelmälíg ober aubgefdrweift, zumeilen efcentsifi, ja ez gift, fogar verwathfene Şüte u. for.

sman verbraudit ten Rufleifjing eben fo wie ben serrenbilfling, won melden in oem näd)fffolgenoen Irtife! cie Siebe fenn wirb. Er iff

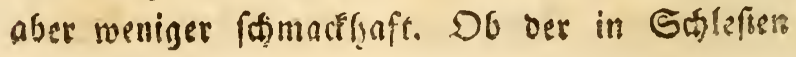


fo beliebte Samalgling hiether geftöre? ift nod burdi) 2lugenzeugen zu entideiben *).

*) Эă fübre diefen Bilgling blok darum biec an, weil er wirflid) allgentein verbeaudt wird. Dennod bale te id ben Serrenbilgling allein für empfeblungs.

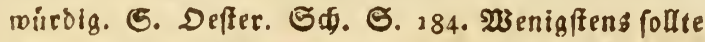
man von bem Rubbilglinge nue junge Studfe gum Genußs ertwáblen, denn tie áltern find f̧on mand. mabt ber Befundigit nadtheilig befunden toorden. Anf der bengefingten $\mathfrak{S}_{a f e l}$ ift definegen ein jün. geres Excmplac neben dem álteren vorgeftentes soorsen. 


\section{Der Serrenbilgling. (Boletus edulis Pers).}

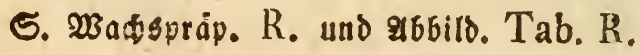

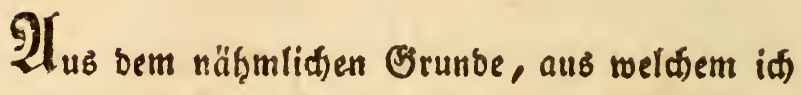
bey ber vorigen Stwammart bie verfdiebenen Drobinzial, uns Rationalbenennungen hinwegge, laffen babe, muf audf hier ein Strides gefde. hen. Dinn bie übrigen Raţmen bez Serrenbilfe

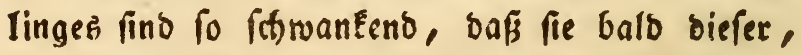
balb aud mehreren anbern 2 arten von Bilflingen beygelegt werben. Sęrenbilzling hingegen nennt

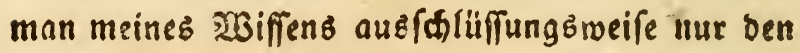
gegenwärtigen. Zuf ben MarÉtplägen :Biens wennt man ihn indefien meiftens nur (d)leditweg Bilfling.

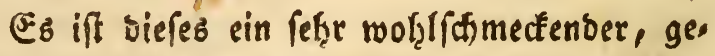
funber und empfeftlungżwïrbiger Sđtwamm, bel weldarm es nur allein, fo wie bey allen Sđwäme 


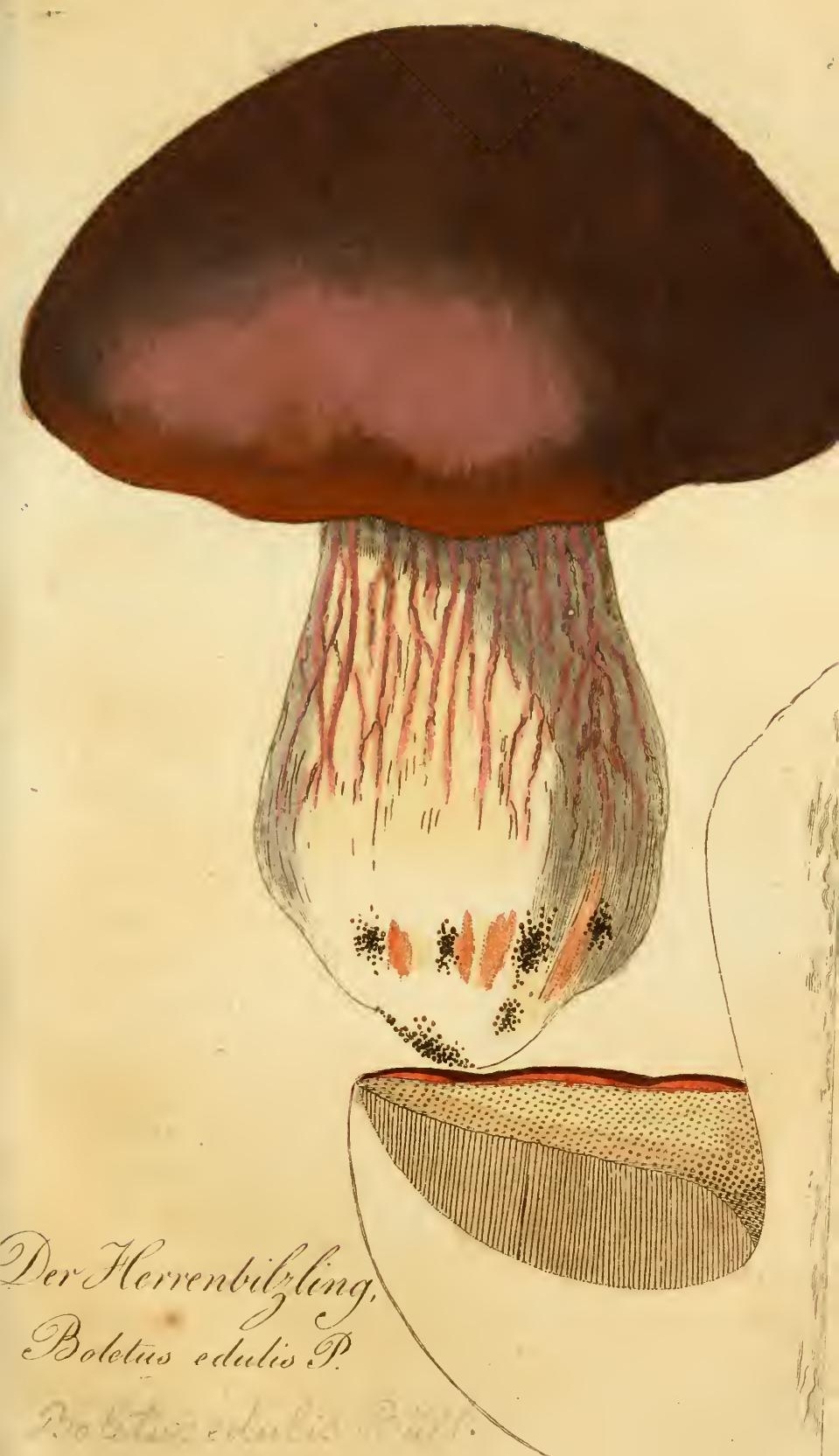



men auf cire fetge genaue llaterfheibung bon ben

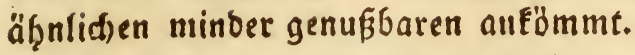

Er räh) am liebften in Thälern unb auf şügeln, in Nabelwälbern und im sceibelanb, zuo weilen aud $\delta a$, wo pelse alte Eidhen in weiten Zrwifhentäumen, bie el̨rwürbigen Zeugen ber reidbegüterten Borwelt, zerfitreut und gleidfam verifheud)t ourch bie Errfáütterungen einez fiüs mif कen Beitalters bas Mütterlide Eroreid) in weiten Rreifen bej(hatten.

Seine Erfheinungszeit ift wom Enbe bes Julius bis in ben Detober, $j a$, bey rehre gelins

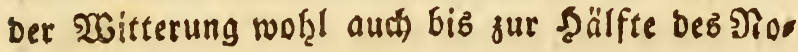
nember.

Der secrenbiljling ift wie ber vorige ein

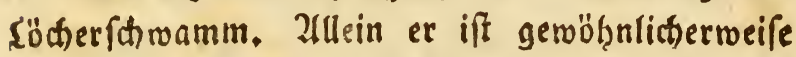
viel größser, bicker und freifwiger. Es gibt Stüs rfe voll ber Sröże eines Menfdenhauptes, obs

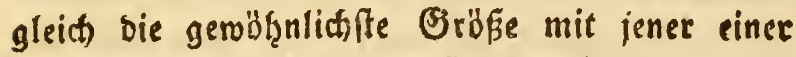
Raffeetaßje zu vergleidjen iff. Er wäd)ft nur felo ten regelmäfíg, fonbern ift vielmef̧r meiftens feb̧r Buctelig, verwadfen und unförmlia).

Es gibt auth) von biefem fo wie von mefre andern Bilzlingen fterile 26 arten, bie swar volle

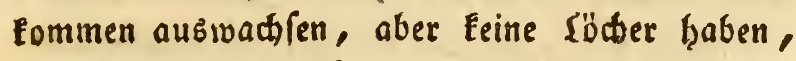
und bie bann feţr fówer zu erkennen find, ba ihnen ber Battungę)arafter mangelt. 2lllein bee 
Sabitur und ber Standort verrathen ihre 20 . Eunft.

Der Setrenbilgling hat einen bicken vollen fleifdrigen 2 big 3 3oll hohen untsn meiftens vers bidten StrunE, einen gepolftrten feţr frifhigen Şut und überangfeine barmadifene bom StrunE abgefonberte Röbraben, bie fich in verbälmif. mäfig sarte, osm blok̉en Zuge faum bemerfbure röher münoen. Die Farbe bes Jutes" if Paftas nienbraun mit einem fefir ounflen Bipfst und lidferent Ranoe. Der StrunE hat eine weibliche Srunofarbe, ift joodh befonbers im obern Thoile mit einem jerfoffenen bem Şute gleidifürbigen Nef̧e bemaḅlt. Sut uno Strune fino überbisf febr zart bereift, unb beybe geben burch bas 2fle ter uno bie Bitterung fureilen in 2 frofgraue

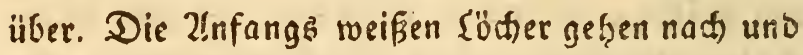
nach ins gelflicte über, fo wie fie älter werocn. Das berbe fileifd ift weif̈, feb̧r fein uno nur ganz unten am StrunEe und unter ber Dberfähe bemerft man cine forrache bräunlid)e Sthattio rung. Das Fleifh Dieles Schrommes läuft nicht ari, uno beränoert niemahle feine Faibe, fondern bleibe immer meiff bis es vertroctnet.

Man mare nur auf bie übraus feinen weifo

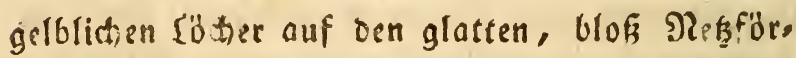
mig bemahiten EtrunE, unb auf die Unwandels barfait bes Derben meifen Flaifdis, und man: 


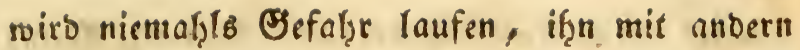
giftigen zu verwedfeln.

If bingegen ein Bilfling Bolbgelb, B̉luts roth, Ziegelroth, Biclet, Blenfarben u. o. gl. bat ber Strunf ein gitterartiges शిę Doer ber. mantelt fith benm Inbruch over benm Durd. fonnitt bas meife oder gelbe glleifh in sBlaue, Slabongrüne ober $\mathfrak{B l e n f a r b e r e ~ u n d ~} \mathrm{S}$ wär lide; fo mus man ifn als giftig ober twenigiten als berbädhrig berwerfen. SM̈̈hte bod bas himm.

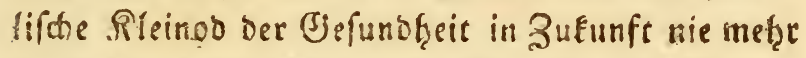
Durd) Ulnvorfictigtert ober Mangel ber Nintur= Eenntnifie verfaleubert werben, o! daf eire un,

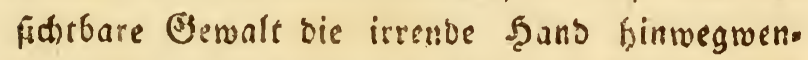
Den möge, die in V̧agriffe ftehet einen Schmamu

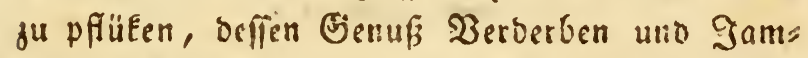
mer im Sthoofe forglofer Familien anrithten müßs. te. Ein harmlofes zllter lobne vem Menfthen= freundicten SRaturforidyer feine Sregenvolle $\mathfrak{B}$ e, müḩung, ber fith mit ganger (Energie uno mit

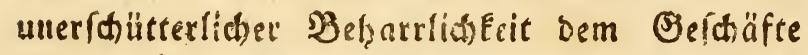
mibunet, bie gefahruolle Dunkelfecit ber SterbliWen aufjubeitern, uno fer mit oer roblwollenden

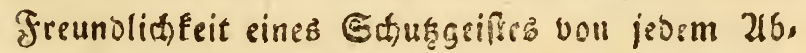
grunde zurück zu byalten, in Dess fie fid whne ihn aus Ilnmiffentzeit fiürzten.

Man nimmt ben fecrernbiffling als Buface

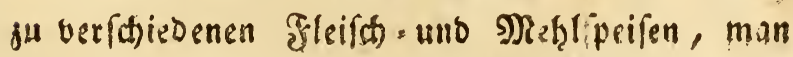


Sünftet ifn aud insbefonbere mit Samalz, Methi, und Fleifd)brühe. In $\mathfrak{B}$ erbinoung mit Sarbellen, Eleines SMufheln und SRildrahtm fann man vors treffliche Serichte bavon bereiten. Man pflegt ihn auch) in Spältthen zerfdnitten aufjutrocinen, und fo für ben 2 Binter an trockenen luftigen Orten

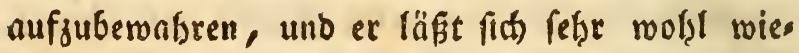
ber aufweiden, ohne viel von feinem $250 h$ lgeo fommack zu yerlieren. 



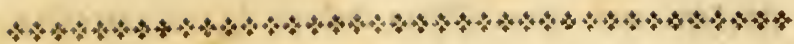

XVIII. Das Schafeiter. (Boletus albidus. Pers).

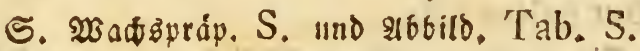

Die gegenmärtige 2lrt ę̧Garer Eđinämme

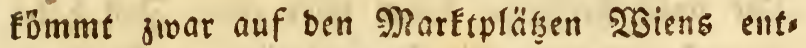
weber gar nictt, ober nur äuferfí felten zum fácin. Da fie jeboct in EtenermarE, Salyburg,

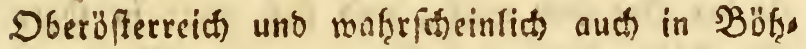
men allgemein verfpeifet riro; fo würbe es eine tabelnzwärbige llnterlaffung fonn, wenn idf) fie

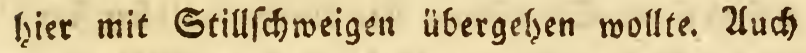
in ben গicberungen ber öfterreichiftien alfpen hao be idf auf meinen botanifhen $23 a n d e r u n g e n$ bies fen Shiramm bäufig genug angetroffen. Man finbet į̂n im Spätiommer in शabelwäloern bes fonbers unter Tannen unb Fidten auf ber Erbe uno man glaubt vom weiten einen Blätter fक wamm (Agaricus) zu erblicken. Err wäh) Rafenartig, aber bie Stücfe felbit find msiftens mit einander, wenigftens ganz unten in einen Sörpar zufammen verwath fen. 2(uth fino biefe äuserft ungleid unt auf allerlen Weife verunftaltet. Es wad) fen näh̆m lich folde Sțrömme von allen Sraben ber Ob̈r̈ße oure einanber; zumeilen fitheinen gleidfam bie 
ftärÉern nub if̧rer s) Ritte andere berborgutreiben, und ein andersmabl biloet fith ein fold)es Shaos bon unvoliendeten Trieben, baf man anfteht, $O b$ man eg für émell Rnollichten Doer Şb̈errid)ten Strunk oder vielmefir für einen eingigen monftrö. fen Şut galten folle.

Fin ausgewaकfenes Etüc mifit ungefähr 2 Soll in ber Sëb̨e, uno 3 3oll im Durd(d)nitt. Der volle fieifthige faum über cinen 3oll bohs EtrunE geḅt oben mit einer allmäḩligen 2lubbreis tung unmerffich in ben 5out über. Sowohl biefer als ber şur fino weißs, und falt glatt in ihrer

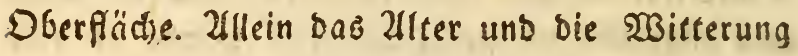
haben darauf vielen Einflü, und man findet zu.

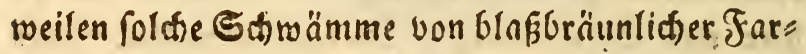
be mit filgigen ober wohl gar mit getäfelten und (c) uppiditen Şüter.

Der Şut, welder in ber Jugeno fal̨r gewölbt,

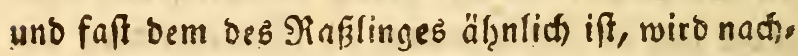
ber in ber Mitte vertieft, faft wie ben einem $P$ fefo ferlinge ober Reizéer. Er ift alsoann nuch ziemlid uneben und am Ranbe ausgeftrorift. Die \{̈̈her an ber llnterfeite bes ફ̧utes find äuferff fart, bem blofen 2luge Eaum fichtbar, feicht uno siemlich weit in ben Strune herab varbreitet. Sie find Ans fangs Sdneewrifi, gehan nber mit sem alter ime mer melor ins gelbliche über. Man bereitet iha mie ben Bilfling, Drehling u. o. gl. 

XIX. Der Eidfyafe (Boletus polycephalus. P.)

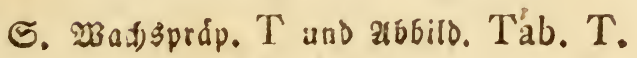

Siefer uno oer nädfifiolgende Sdimamra, melo when man aud) Eidfigafe nerrint, fino von fo mancten übrigens nime unbebeutenden Esdrifts ffellern mit einander verveedfelt morben. $D 6$ num

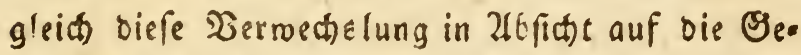
runbfeit Der Menffien von aller Befafir entfernt iff, indens bende eşbar find, fo bleibt es boct immer ein Grtthum, uno Grtthähner müffên nies mable vernawiläîigt, fondern aufgeflärt uno bee ridtiget merben! Denn wenn fie aud an uno

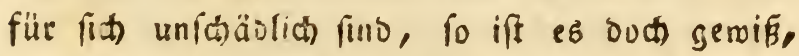
Dafs fie nach uno nach immer mefrece an fich zielgen, und enditi den ganjen Berfitand Derjes 
nigen, bie ifnen frenwillig anfängen, virwirs ren und varbetben.

Dor Eichefe erreidt Érineswege bie Brös

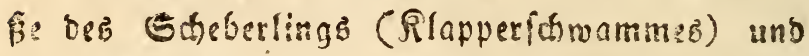
feine Gruneftengel unterftügen bie Şüte in Eers trum, feine Shite finb oofie: gand und nidits wes niger alz Dadjigigelförnig üter einander liegerio, und aufgethürmt.

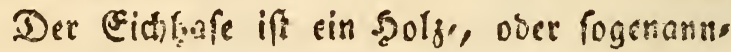

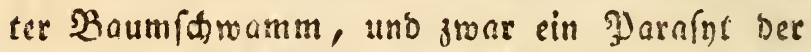

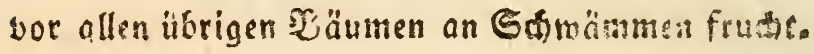
Baren (sid)e. Io fab ifin in ben unermeflichen

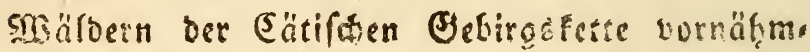
ridg grifden Maurbad uno stseiting. Esine Erfochenungajeit fält in ben Eeptember. Er ift baher auth bierinn ton Dem Rlapperformamme unterfohieben, fo wie er tibrigens aud viel fels tener ale viefer if: Man firbet iffu vorzüg.

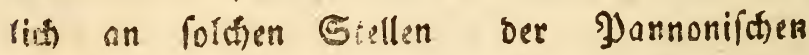
Earreite (Quercus Auftriaca Willd:), mo dies fe burch Stumminge ober Menfenen verlest mos, sen und beträbtliche defte verlohren baben, fo

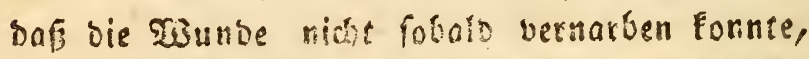
bevor now Regen, froft und Sommerbitige eine Föulnifs in terfiben ergeugen mub, bie nur alls zubalo von Snfelten gerwittert, who von Den ib. nen nawfellenben Specten nod reiter befös bert wirb. 


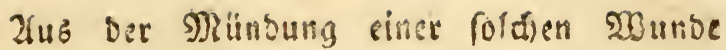
bringt eit monfröler Gtmammfötper petoor, welder zumsilen melerere Y) funbe ftwer und falt

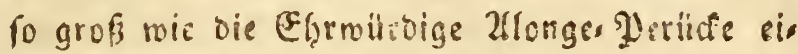
nes altfrianfichen Doftors wirt.

Diefe harborrajende meifie und berbe, finsts

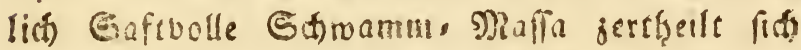
obne allar Dronung, tow meifiencheils nad aufo

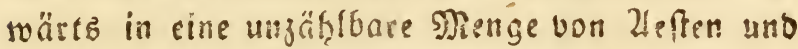
Zeftcticn, und die Siatur zeigt hier gewiffermafs fen einen lupuriojen und faft aueformeifenten Şang zur Drolififation uno ßervielfälrigung oet fpecifien Formen. Sleish bem Fabelíaften Une

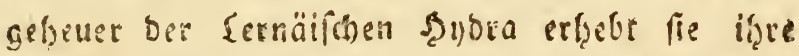
Sकlanganbäligen Säupter im sidten Georänge, uno mann (dion bie Sy:nge Derfelten fith wed). felfeitig im 2lufridten binocrt, fo bomerft man

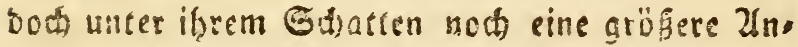

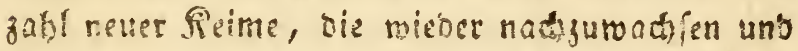

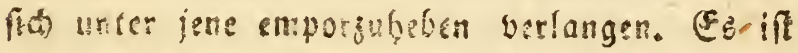

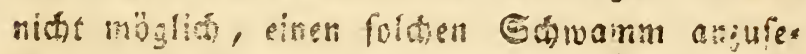

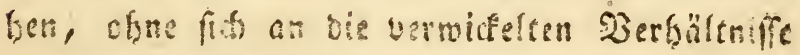

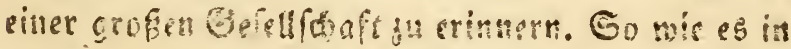

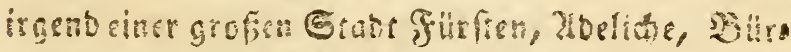

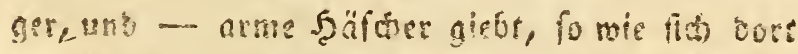
bie Grofen mit ibren 20pithen umo 20 irfungen

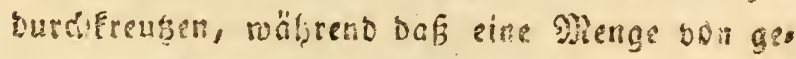

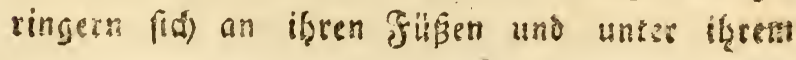


Sharten emporzuerbeiten trantet, und fo wie Dirfe vergुeblich nach Sröpre un' Sicicithümern ringen, weil oow unmöglich alle Menfísen Für fon fenn Ë̈nnan, uno meil es toeder Ef̧ren noch Reichthümer gäbe, wenn biefe einem jeoweben su gleichen Theilen auggemeffen wären; eben fo zzigt uns nuth hier Das Bilo biejes Echmam.

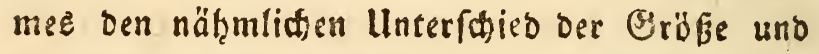
ein ganz gleiches ßerfältnif́ ber Niebrigen zu ơn Errhabenen, unter Deren Sthuse unb von beren Uleberflufi fie zwar fpärlid und berbor gen, ober bock glüclich und nidt menigar bauers byofe als bie übrigen alle vegetiren, obgleich ifs Sauptfanm es sicht vermag, jebea einzelnen Reim bis zu jener 20 dlpänoigfeit ber Erffges bobrnen zu entmicfeln.

Der Scauptfamm theilt fict bemnat) in fo viele gröpere uno Eleinere 2fofe, als nur immer neban sinander YInt baben, und biefe mieber in fíeinere u. f. w. Ifm Sauptín amme fowohl, als an oen Veften und Zweigen fiebt man unjäblig viele Triebe uno gleidfam Rnofpen von Schwäm, men. Sie fino ütrgens, wie fon gemelet worben, ofne aller Dronuig oer Sröfe, Form, uno Ritung ourheinanber geflochten.

Jeber 3reig enoigt fich in einen regelmäf. figen, anfangs gewölbten, nachber faft Trich: terförmigen fahien sut, weldor in feinem Een. 
trum aufikgt, und aus einer 6lofen Erweiterung ses Stieles entifanden zu fenn foreinet.

Oft mifrätl) bas ganje Degetabil (ver. muthlich wegen übermäfiger 2lustreibung ber Fructiörper ober Şüte?" uno in biefem Falle ift es fich nint zu wunbern, wenn je irgend ein Dotanograph gezueifelt bat, gu welcher 2 ret et Diefe Sanmammähnliche Mifgeburt bringen foll te. Dir Seüte find bann faft ton \esizen ähn.

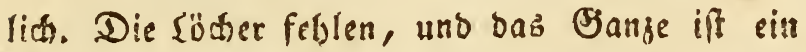
Reulenfomnmm (Clavaria) mit ben Extremitäs

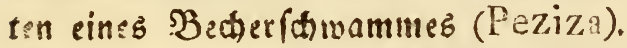

Sslange jeboct) Der Sdruamm zu feiner Rei, fe, fo erbalten bie oberften fäte ben Durdimefo fer eines Bolles, uno biefe fino in ber Mitte vers tieft unb foft genabelt, am Fanbe hingegen ets was aufgebunfen uno eingerollt. Die meifie Un, terfläche aller site ift mit febr feinen, bem frenen luge faum fichtbaren, nidit feldr tiefen

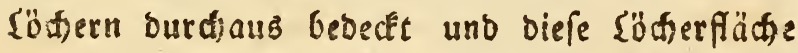
zicht fith weit, ia faft bis auf bie Sälfte ber Stiele berunter.

Man bringt ifin nur fefre felten ou Marts te, ex ift gefuno uno angenefim. Man brreitet ifn in cen Sïchen wie ben Bilgling, Drehling. u. D. gl. 


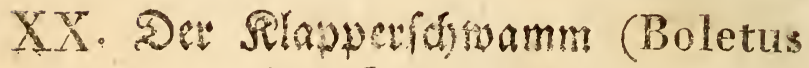 frondosus P.)}

S. SBadgurap. U und asbilo. Tah, U.

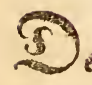

er Rlapperfitmanm, Ehoberling, Etrepers ling, Eishafe ift zwar dem Boleeus imbricatus oez Bulliard feter äbutioh, neer bennoch von ihn burd Siandort, Jafrejeit und Eigens

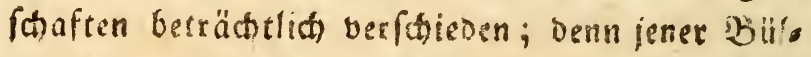
liarofthe föhtherforam fömmt fhon im Man in einer Söjge von 40 , Fuß an Den SBäumen zum शorfiein, ift bitter, ungeniefbar und frat ben Serúa voa ist Enjianmurgel.

llifer Rlapperfoway Ë̈mmt aufer Des fierrein, fovid bisfer bavon befannt gewotben, auth in Bancen, Ungarn uns in Englano vor. (E) it efbar wie bar borige, Ëomint aber nur felten fu Marfte? 

Den 9ufomen Stheperling muf man von bem Dberbeutftien Beitworte Schepern ableiteal, welthe fo viel heifit ale Rlappern, ein Sagels regen äbnititise Ocräufich barvorbringen, ver muthlich weil bie vielen Şite benm SBinde ober wenn man den Gaframm mit der Sand folit: telt, auf eine ähnliate 23 eife Elappern coer fhee pern, inbem fie an einander froffen, und zitserno zuriicfprallen!

Dafí Elufus diefon und nitht ben borigen Eđroamin beabfichtigt habe, exf̧ellet baraus, weil fein Saroamm im Spätherbfte an Den 2Burgela Der Eichen hetbortrsibt, DunEstrotf. braun ift, uno Dadfienelförmig übersinanber

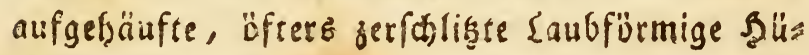
te bat.

) Man nannte ifgn zu feiner Beit in llugarn Bokros gomba und hatte oen 2 Bahn, onf er, wenn ein Reifenber vorubergienge, und if̧n bes rounberte, plösclich - aus Eiteítrit - ju einer ungef̧euern Sröbe anroahfe, ja man fagte fos gar, ez müfiten ganje Şefellifhaften Zugroeife zu if̧m in ben $\mathfrak{M a l b}$ manbern, wenn man if̧n rectit grof haben wollte, benn er würos immer befto gröfier, jemafir er Bewunberung erfïlcre, im Begentheile begnügte or fid mit feiner ges

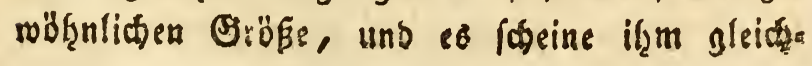


fom bart gu fallen, menn er fin won ben Men, folen vernachiäf gt fähe! Sgan Eann aus biefer Fabel mit treffender Serwifheit oen Geniub der Damabliyen Gaten, und Die Nationalanlage iḩ rer E. firo:er erfennen!

\section{Clufue fagt ferner, man babe ifm erzäblt,} Doh man bon diffm Stromm gumeilen fo gros

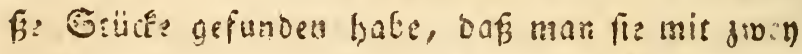

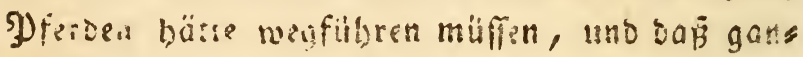
se fomilien oon Mnfetophagen bavon fith fïts ten lait ffen törnen; toch fen Der grögte, ben er in liraarn gofeten babe, nidet fo grof geo mefen, obglict aibfer, nle er is einen anbern

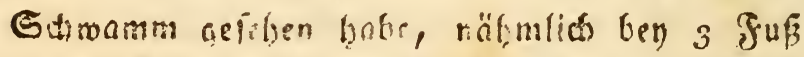
in oar seöbe, uno es rätten mobl 3 ober 4

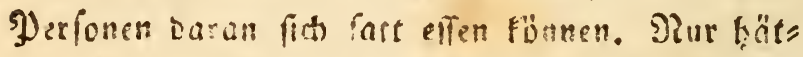

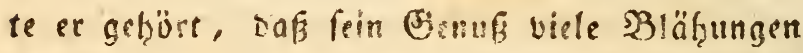
veturfactret bätte 1 . ?. m. In der That bate aud id nos Erinen foldin Rriren von Sthwamm entlictet, obigleid tie gröbren Stücfe vom Boletus citrinus, dic iá) zu melt habe, ihm sienlict natse famers, unb eine von mir beobatototz Daetalea Quercina cine länge son mebe bainn 7 . Slaftern errsichte, bie jeoch) nirgende mehre al 2 3oll aus dem hol. zo hesvorragte, uno bafier nur gleichiam eine lange Binde, nidit aber einen groken ftarfen Sỉiper batftilte. 
Der Rlapperformamm gleichst ram Eid). bofen, ben er an Gröbe noch übertriffs, in oer Sielfältigfeit ser 3eräftelung. Zus feinem oidfen, faft fuffangen Etrunfe entpringen melprere bun

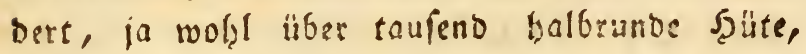
Die a if lauter aufgerithteten Stielen mit ibrem Siande feffingen uno in girider Yidrans ubers cinander aufgethürmt, ietod nur locfer Dadio ziegelformig fio einalsber bedefen uno befhato

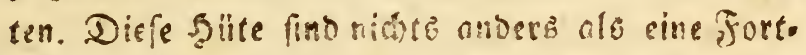
fersung und Ervalicrung Deb Gtrunfez, uno

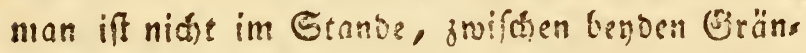
den anjugeb:n. Sie fint bon Sefralt halbrumb, unregelmäbig jertheilt, ober Manfotettenartig gefalten, uno zumeilen faf bem sïrren Einens laú ähnlin. mit welcicm fie auth bie broune Farbe acmein haben. Shr Durrimeffer iff ill 1

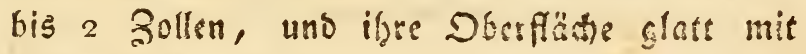
corcentrifitan Fibern.

Die 〔ö an oer weiffen llaterfeite oxtels ben find eben fo fein wie ben bem borigen, uno reides eben fo weit an in Stielen herab, ja faft bis an bie Zertheilung ser Brorige.

Ere wädift in niebriz gelegenen fauditen maloungen an ben $\mathfrak{B}$ uryeln ber Fichen ober nahe on ber esoe, uns wirb ba von ben Enmammfammlern in DFefer aufgefuchet. Er iff trog foins: berufonen Eitelest ritht gecignet, 


\section{2}

als ein eoles Berüdt alif dis Tafeln ber Bors neţmeil ertroben zu werben, woţl aber Eann man if̧n als eine शabrungefpende für bie aro

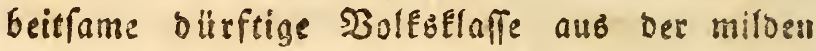
Jano ber Müterliden Ratur erÉnnen, ber, gleiden fie fo viele zur 2sushilfe in ifrem 2 ors rathe aufbebält, un unfere Nïhfeligfeiten im פothfalle zu rinoern, uno aut im falimmften Falle einer verunglüten arnote ben Sthmad). tenden now ein \{abfal für if̨re Bebürfniffe an

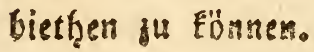





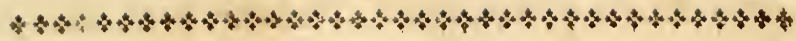

XXI. Der Sleforfinamm (Boletus (Fistuliua) hepaticus P.)

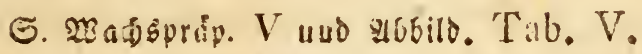

\section{9) P}

an hat in Detutfen für biefen Sofromm axd nod) folgenoe mir bsfannce Benennungen:

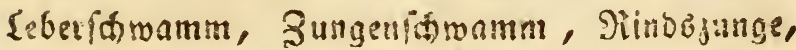

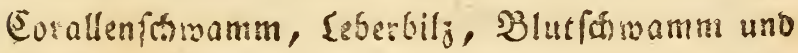

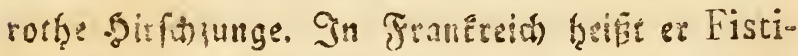
line Langue-de-boenf.

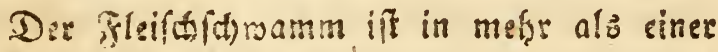

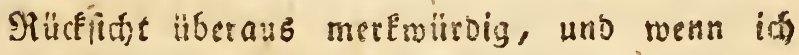

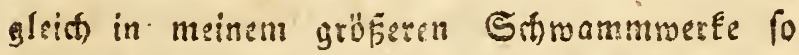
marthe $\mathfrak{B}$ argleithung angeftell habe, wm feime Infínten zu verelären, fo blieb mir bennod now einig und anberes ju bemerEen ïb:ig, weldises

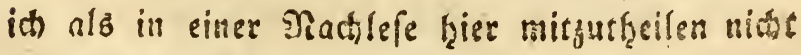
fiir llaberfu! balte. 
Mander lebt vielleint in bem eiteln 20 abn, bafi unfere Saturfyftene uno unfere Genera et specie: p?antarum wobl zum minteffer auf eben to fefren mo raustgafter Furbamenten wie bie tatifenciänrigen Eoloffen legyptens ruben, uno nur böbftens in ber Fraçade nod) bie uno ba eimpr 2lustefferung bebüten. 216 a a , wie farne finb wir nod bon ber Wirflidfeit riefer

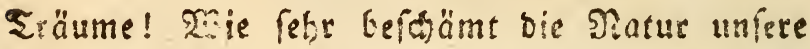
Sirngefpinnfiz! stie oft verfpotet fie unfere tiefinnigften SpeEulationen burch bie unermar, tetfen Erftisinungen! Srabrlich! die Methodi ften Der Saturgefdidte würoen meit befier ges than haben, wenn fie anfott ber Mavortifchen Sathination lieber sem Senfipiele ber Sternfuno bigen gefolgt wären, uno nur getreu nahges griçnet uno eingef́laltet bätten! Denn wenn auth in ber Sternchate noh Sterne fehten, bie nod) Fin Eefrrohr erreidte, fo find boh bie beobactiteten alle an ifrem rectiten Drte und in ibren riditigen Berb̨ältriffen! Zllein weit anbers verf̧ält es fich mit unfern fnftematifden Nature gemäbloen. Einfeitige Beobadtungen weroen zu aflgeneinen Regeln exboben, unb boch giebt es in ber ganjen Siatur Eein Berbaältnif, Eein 2 t,

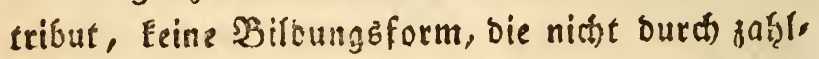
reithe Stufen in anbers übergienge, unb fith wie Etrab̨len aus cinem snittelpunte nath allen. 
Sithtungen mit ien 26fufungen oor andern Durdireuste. MRar barf fich alfo gar nidit wun. Dern, wenn heute neue Esattungen surch Zers theilung der ältarn erfíaffen werben, die mors gen ein neuerer Botanift verwirft und wieber bereinigt! menn biefer jene, und ein anderer wien ber andere slaffen hervorbringt oder reftingirt.

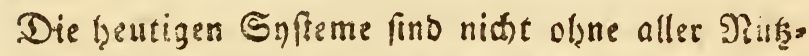
lickett; fie entipretien einem Theil unferer $\mathfrak{Z}_{\text {es }}$ Dürfniffe, aber fie tragen aum alle das Seprä. fe Der Denfolid)en Unvollfommentzeit, uno if̨. re 23orzitge fint nur relotio zu oen Bseifesfä. bigfeiten ifirer litheber, aber in Sginficht auf bie originelle Natur fino fie alle glcion binfüllige Suftgebäube!

Der fleifofformamm bient un treffliden Exampel über bie Teffigéteit unierer Sattungstaraftere. Er if ein L̈biterformamm, benn feine Unterfeite if wie bie der übrigen $\{0$

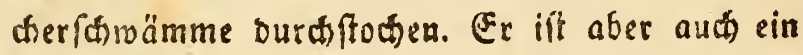
Stanelforramm, benn er treibt allentbalben

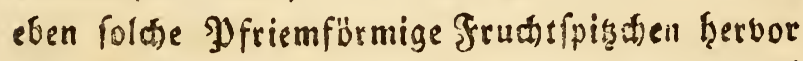
roie bie Stathelforämme, uno nue ein Theil berfelben üffnet fith an ingrer Epiţe. Er ift eine eigsne Sffmammgattung, Denn reine

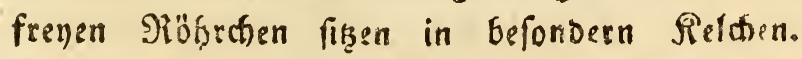
aber biefe Relde verforminden mit bem reifen Alter! in oer Jugeno find fratt oer Rïhrden 
nur Srübonen bolf̧anden. Es ift saher cime an. bere Sattung in Der Jugend, eine anbere im 2liter, sine antere auf oer Unter. und rieber cine andere ouf ber Dberfeite! Toe! ఉer bon bic. fen bieren wollen wir ben Jु)eifi fuertennen?

$J$ (h) Fünte fefre leids, nithr allein aus ser Familie der Sobrömme, fonbern wohl gar aus Den Dhoenerogamifán (Slaffen eime nid ganz unbedutero? Gumme ähnlicher Renfpiele ouf. fillen, allein eb iff bick niát oer Ort bazu! Nur fen es mir erlautet, meine sefer für biefis mal an bie bereits ziemliti) of bamerten गुes lorien, an bie fortwährenten 23 cränocrungen in Den Fnmifien ber Farren, Der Moofe, der Z2Baf. feralgen, Der Flodten, ber Sonnämme, uns an bie facale llnftätigerit ber Gattungen: Alyr sum, Andropogon, Astragalus, Calamagrostis, Chrylanthemum, Cucubalus, Diosma, Diospyros, Epipactis, Euphorbia, Gentiana, Geum, Hydrangea, Ixia, Ligusticum, Limodorum, Mimosa, Myagrum, O, hr s, Orchis, Paffiflora, Satyrium, Selinum, Se. rapias, Swertia, Tormentilla u. b. gl. zu erinnern!

Ez ift cine fincre 2ufgabe, ben Fleifds formamm zu beforeiben. Er if fo regellos, Daf

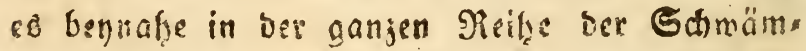
me Eeine Geftalt giebt, bie er nicht anjunet? 


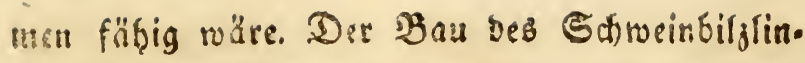
ges if ihm fo wetig zurbiber, als ber bes brähtigen Stammpilzes (Boletus citrinus). Jandimal ift es ein förmlider Reulenfónamm (Clivaria) uno wieber ein anoeresmal gleidt er einem faltenfinmamme ober einer Siorde! Er erreict sie Gröfie eines Ropfes, uno bat 3nuelisu Eaum iber einen Boll im Durdmeffer. Wontier unteftimmt iff feine Farbe, benn dies fe ift meifentipeils im jugenoliden Ulter cin balls bräunlides Rothgelb. Er miro nadher Blutroth, who gefte mit ber Berrofung immer meti: ins Edrocirgliobe uber.

Eein fioper mag, nun aber was immer für eine Seffalt annelimen, fo bemertet man boch allemal bey bem ?iafwerten Deffelben cine rauhe warjichte Dbeifiäte, unb biefe 2 sär wen, genauer betraditet, fint Eleins bohts Ens linber, ein isoweopr anfangs ucn eirem häutis gen Szlumen. Doer Straformigen Sielore ums fangen. Gis watifn nac) unt nath fort bis ju eincr länge von bren ober vier \{inisn, und fres hen bann auf ber llnterfeite gebrängt aneinan. ber, uno dis ficlde verforwirben. Zuf ber Dbers feite hingegen forinen manditerien Sinderniffe die Entwithung biefer Röhroben zu verwebren. Jä ne öffinen fich an ifier Epiz̨s, und berfanfin

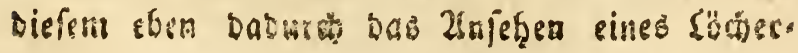




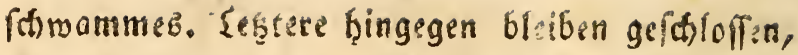
formupfen kufamman und geben oem Stimamm auf reiner Dberfeite ein Filzigez Anfehen.

Sein Fleifin ift rebre feinfarericht und eto was meich, jeoosh oidt, und giebt, im frifhen Suftante rurchgrftnittet, ein felcr reijentos Eaquficl. Die Grundarós davon ift eill reis nez belles grseif mit mancherlen Rofenfarbanen 23 cllen, vicleten Sügen uno gelbröthlithen 200 l. Een. Zilleia won bim exfen Zugenblicte an, ba ro Der frenen \{uft ausgeftellet ift, vermanbelt ce Diefe 5)ableren in cine Dunflere gleitfartige Färbumg. Ditht nur diefe angegebenen Zeichnun. sen, fonbern bie ganje Dberfähthe miro nach uno nath benfarten oore formarzroth, fo bafi

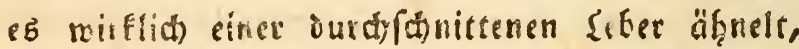
und alfo ven Sabmen des Lebarfánammez gan? uno gar rewistfertiget.

Man findet diefen Gofruamm in feuchen aber bennod) rarmen Sommern an unfern (E) chen, uno zroar fowohl an frifolen, jungen und gefunben Böumen, als an alten uno abgefter. Eenen, ja fogar nuf oen abgefägten 23 urzelfiö. ofen ber gefülisen Eidsen. Mran firbet ihn in bes trärbtictier Söbe won 3 tis 4 Silaftern, uno oft wieber faft gariz bey dem Boden. Der 24 .

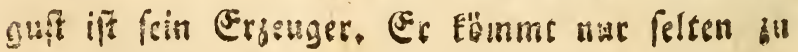


Marfte. Man hat Ben!piele (đäbliđer WBirfuns

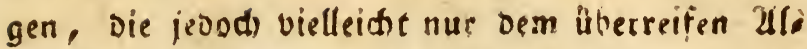
ter, und bem parafncticten Ungegiefer zujufthreis ben waren.

Man getrieft ifn als Sallat unter Rräus tern in Spältchen gefdtnitten, Dod 'Eodts man itzn aud wie ben Sillzling. 
XXII. Der Shabittfdinamm (Hydnum imbricatum $L$ ).

๔. Bađspråp. X und \{tbbitb. Tab. X.

D) iefer Stachelfómamm, meldien bie B̉ayern braune Sirfäjunge, bie Floriffen aber ziegelar. tigen Stahelifiramm oder foruppigen Stanel, fatwamm nennen, ift mir nod niemafis zu S $e^{\circ}$ fitit gefommen. Da er aber in ber Saljburgero

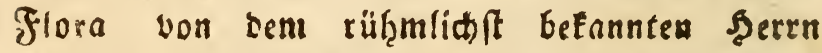
Braune aufgefüfreet morben, und biefes \{and nunmegre ben Defterreidis = Raiferlidien Errb= ftaaten if eiriberleibt worben, fo barf er auch hier als ein effGarer Sdwamm nift mit Stilld. dweigen übergangen reerben.

Gein Gtrunk ober Etengel ift feft, boll, in ber Dberfäat)e faferidst, un rein meíf, unn 

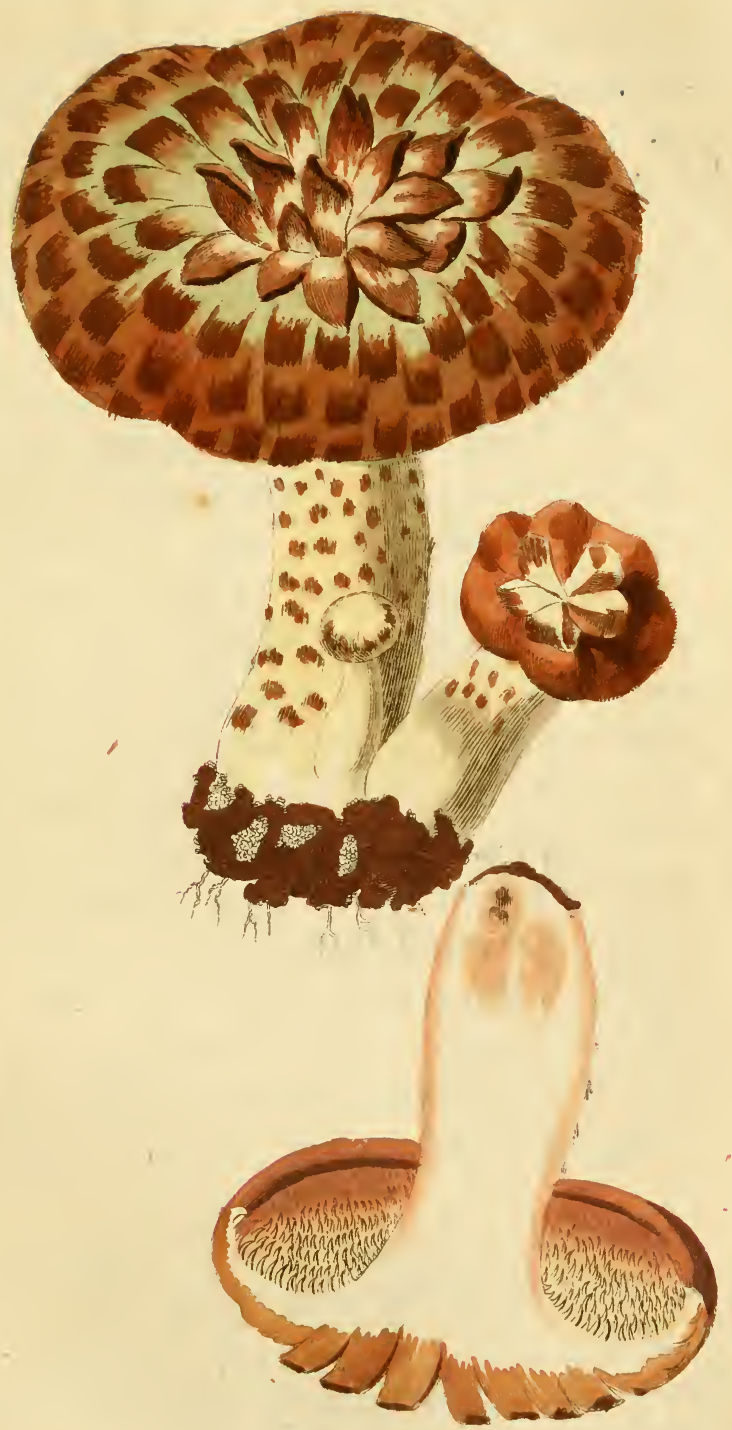

Der Ftabichto chwamm.

Hifdnum imbriacutum $P$.... 
132

mandimal fo georängt beyfammen figzn, bas Daraus eine 2lut won Dach entfelit, invem fict die Säte felbit Dachjiegelförmig bebecten. Ilro es ift fidmer ju entfheiden, ob die Senennurg Des Dadziegelförmigen Stadelfdrammes mehre von diefem llmftande, ober von jener SBiloung ber S(t)uppen herguleiten fey, die id furg vore ber befarieben babe.

Der Sabidtictroamm roirb einftimmig als ein belifater, effbarer uno gefurder Schramm gerüfsmt und er foll vorzüglic in Jtalien frare genoffen merben. Er ift in verfobiebenen Begen. Den Deutid)lande, in DänemarE und Enaland zu Saufe. $O b$ aud in Frantreich, ift nod die Frage! bena Bülliards 36 billoung forzint einen ganj andern Stachelftroamm vorzuftellen. Fr fönmt im September in शabelröloern zum Borfónein, und ift ein Erofdromam. 




\section{Der $\mathfrak{g} g e l$ (d) Erinaceus P.)}

5. פEađgpråp. Y und rubild. Tab. Y.

\section{(9.3.} egenrärrtige Sdimammart verbient es, untee Die ếbaren aufgenommen zu werben, wenn fie irgentroo, wie id vermuthe, häufiger vorfom. men follte. In Defterreich felbft getjört fiz gware ou ben gröBeren Seltenbeiten, uns id habe fie nur einmal lebeno zu Beficht befonmen; ; allein in Rärnthen, Eroatien, llngarn und Siebens bürgen wiro fie wabiffdeinlid viel bäufizer er. 俰einen, weil es ba viel mehre sidtenwälber giebt, als in Defterreid. Sollte fie bemmath bort nod) nicht zum Sinu占 angewendet werben, fo Eann idi) Den Einnofnern iener Staaten bies fen Sdwamm als sin neuse Naf̧rungesmittel 
empfehlen. S2ac) mener Unterfúdung bat er

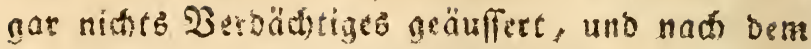
3 unniffe cinse Bulliaro wiro er miteli in Frane. reich vornähmíd in Sorbringen böufig genofien. Man hat oen Jgetionuamm biş⿻er auffer Diferreith un' Frantreich nuch in Englanto ges funben. Bupbaum fans if̧n in ber Europäifden Türey, in Thracien ober Romarien bey dem Dorf Bolgrab an einem Raftanienbaume, uno Ecopoli hat isn aus bem Sápoz ber Erebe her.

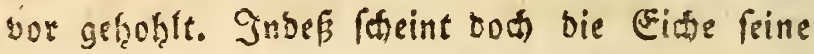
rahre uno eigenthümlide Seburtzfätte zu fenn,

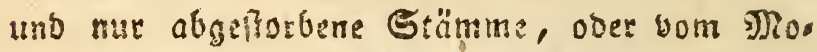

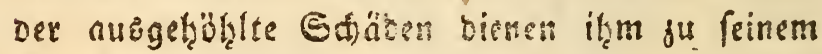
Stantorte.

(F) befteht aub einct Sthuemeiffell berben Shaffa eines jiemlich faftigen, meiften secriför. migen Sditonmmförpere, befien Seruct ref̣r (t) wad Sdroammartig, aber gar nidt unanges thtsm iff. 9Mandimal if e: mit etras tieferen 2ushöh̨!ungen verfeben, uno hat baber fürmlis the Serjobren uno Serjigigein. (Ex ift ourch uno Durth von gleidier Befhaffenteeit in oer Gub.

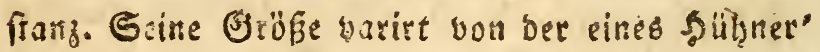

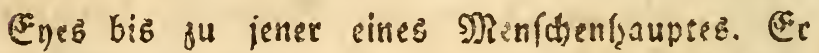

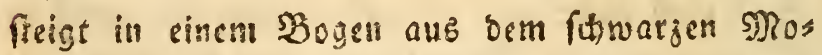
ber feinte Standortes fietsor, if oberf̧alb am meiften breit und sh habca, uns verfatmälert fich 
nach abwärts in eine fentrechte frumple Spitze. Zumeilen, (menn ex näḩmlich) aub einer beträ由t. lichen Tiefe emporjufteigen geribthiget ifi) billozt biefe feine Stüf̧z eine 1 rt von StrunE ober Sals, bie lith jebont, fobalo ale fie bie Deffe nung erreidhet, alfoglecíl in bie gewöhnlidie frorm bes Sthammes aubbreitet. Der ganje Rörper biefez Sw wammes, fo weit er ofien uno fren ftht, ift mit einer gebrängten Menge

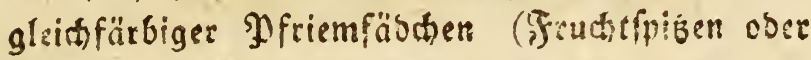

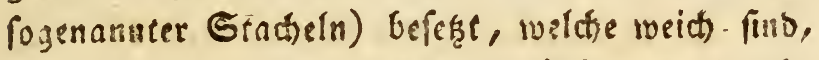
und theite gerabe, theils verfotungen herabs bängen. Siad ber 2lingabe bor Ethrififteller frino biefe fänden hohl, unt obgleich ich baz Ber gentheil beobachet habe, fo fineint es bow, baf mrin Ex mp!ar vielleidft nur zu jung war, und baf fie erfi im fpätern alter biefe $\mathfrak{B}$ ?

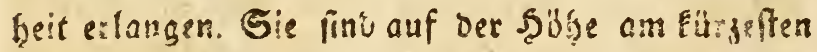
unb viel foltiterer, auth meifentheile aufredit. Ie weiter man fie nad abroärte verfolgt, beffolänger und befto oebrängter fint fie zu Gemerfen, fo, Da B bie unterften faft ric \{änge sinzb Bolls erres then. Der gange Sotramm fectl batier von wetrats einen reifien Bart vor, walder wie angebeftet won bem buneln Orunbe feines Stanborté for frappant an ber Eict)e herabtzängt. 


\section{6}

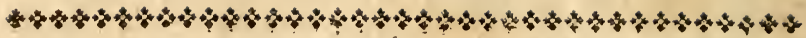

XXIV. Der Corallenformamm (Hydnum Coralloides Schaeif)

5. Wađłpråp, $Z$ und 21bbild, Tab. $Z$.

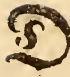

ar Eorallerifónamm giebt ans ein neues Penípiel jur Belztrung über bie Unficherbeit Der bisher angenommenen Sattungömaratere. Jrenlid wobl nähert er fith nad bem erfen

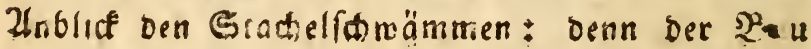
reiner Grudtefpitaen if yon jenem ber übrigen Gtachelfdrwänme gar nicht verfotieden, uno ber eben vorher befhriebene Igelfiframm bils bet einen youtreffliden llebergang von ven Sta. कelidfrämmen mit einem Şute ju ben unbehุus teten Straudartigen. SEenn wir nun aber ben gegcumärtigen Coraflenifitwamm genauer betracto

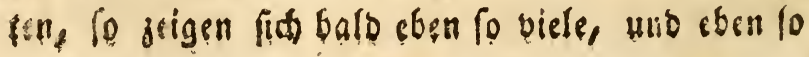





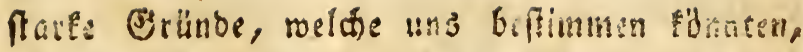
inn ben Elavarien beasujählen. Dean auffertem, Baß̧ ber gange Bau mit Diefen vollommen iber,

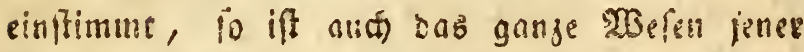
Stadi)eln nidt to weiter alo cine Beräfllutig uno Berthellung bes Enmammez. Dis Stache!n an

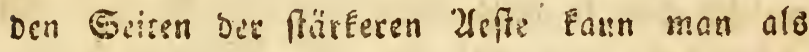
unvollenmene Irebcntrite wie isne (3)ro, 19)

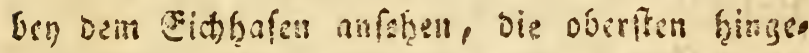
gen an oen Ertrcimisüten ork 3rocige, als die

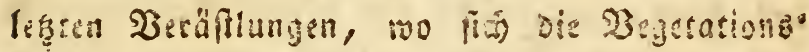
Erafe Der gy/fanje bersics erföbpft bat, uno bas

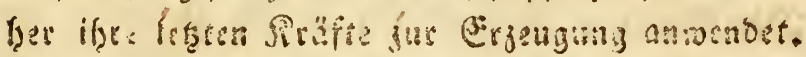
Solle man aber ous diefera Grunde Den fene lenformantn (Clavmia) mit bem Etanelfarmante me (Hydnumi) vetsinigen rollen, fo würoen

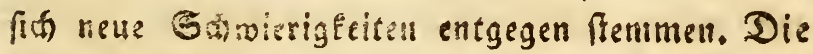
Mittelgefthopfe, bie ben Eorallenifwamm mit Den übrigen Stachelfwrwämmen verbinoen, ver, Eesten biefe Mefen fo feft untereinarber, bas man enblich auci) ein Hydnum repandum mit Der Clavaria piftillaris in eine Sattung verbin. ben müßzte, uno wenn man bisfes eingeten molls te, fo räre zu bejorgen, oafiz am Enbe vielleiche nod) gar alle Sthwammgattungen in eine sinj:

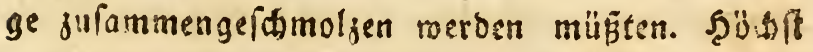
merzioürbig uns widtig fins in ber Familie ber 
S丸ruämme bie zahllofen Mitteloings, wie 3. lene, weld)e bie Blätter (herfátwämmen verbind:1!. Oewiffe Urten von Daedalea, Siftot:ema, Merulius und Thelaephora fheinen beynatze nichto reeiter als un. vollemmen gebliebere Sdfmammaren zu fenn, welche eben fo viele 2 nfprithe auf bie Sattung Agaricus, als auf jene bes Boletus haben. Helvella, Morchella, Spatularia uno Clavaria haben ifice Iften, bon benen man niabt entfhei Den Eann, in weldfe von biefen CHattungen fie mit größerem Rechte gejäḩlt merben bürften. Die Bjattung Phallus verfïatt fi zu biefen ges nall, wie Amanita ou Agaricus, Mit einem Worte: es fheint uns now ganz und gat an jenen Beobact)tungen zu feţlen, auf beren Ẻrunb wir unfere Battungsharaktere bey ber Familie ber Strömme flühen bürften, und bie ange. nommenen fenen onher blos willEührlich) und nue sufs Serntherwohl erguiffen, um bod unterbef, fen nidit ganz unbeholfen zu fenn, biz unz ber

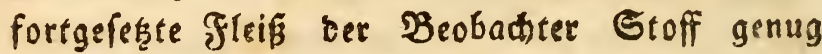
liefern wirb, aus weldent fit wahre und in ber Natur feloft gegrünbete Sattungen bilben laf. fer. So lange wir aber now) in jener grefien Unmiffenţeit verweilen, welde uns verhinoert,

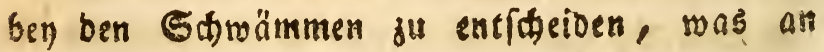


if̣nen refentliche Billoung fer, uнb was bem Einfü bez Stanoortź, ber IDitterung, bem Elima unb anbern zufälligen Ilmitänoen juges fhrieben merben müfe, fo lange werben auch unfere Sdruammgattumgsn Glofie Inmafinugen und grundofe Berfuche fenn, und ein Enfem

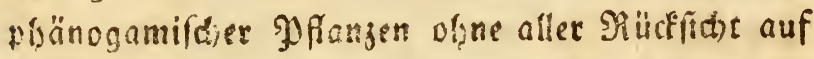

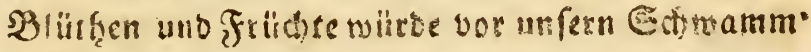

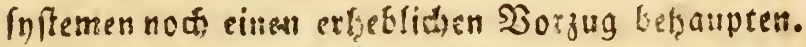

Der Eorallenformamm ift unfreitig eireb ter fönften Dुrobufte Hon Dem Gebiethe ber Garuanme. Bennafe toie cill Loranthus ober Viscum wädif an ben Stämmen, aber nod p̈fter in bem Mrober befhäbigter, ober an ben

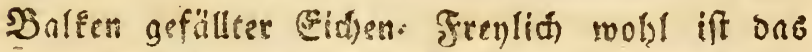
sigentlite Begetabil bes Eđmammes in ber ver, vefrnben Maffa ses SBaumez aubzegofien, unb alio gang ein anberer sharafyt als jue genann. sen Pुgänerogamen, aber Der fervortrsibenbe fidtbare, jur Fortpflangung beffimmte Theil bef folsisn, oen man in gemeinen \{aben oen Strwamm nennt, biefer forint allering fait eben fo rie

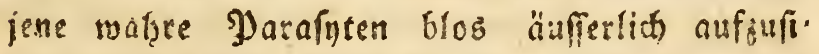
ten, und fid von ben Säften beg Saumes ernäbren. Sein runcer fisiffiger Souprftamm bot zumeilen einen Durdimefer bon mefreren \$ollen, fertheilet fit fiboth gleirb nath oem Urs 


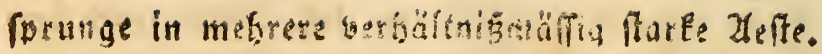
Gromm und Iteft bobes wor Ilufen bie blaffe

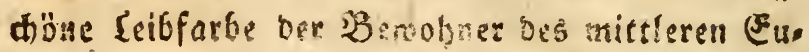
ropa. Gn bent umgetebren Sistraltmifle, wie bie Jefte an ber 3abl zurefhmen, nefrmen fie an ber Dicie ab. Sie erbjben fin in aufo freigender Richtung, utb filden eine folde STenge von duariomofen, bofín wir baburch an bas Berippe bes gemeinea Gitterfohmammes (Clathrus cancellatus) erinuert meroen. Zuturn Die Eptremitäten find fren, unb bilden and in

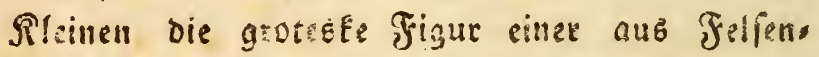
wano frief Gervorgerwadienen Dahfürmig vero Grettetsi Tögre von weitjem Elfentein mit gols Denen Padein. Er mädift bemmä, bio auf bie 2lnafomofen, ganj Straudhartig und biloet eine föone, in matgere Dyarthien abgetheilte frone,

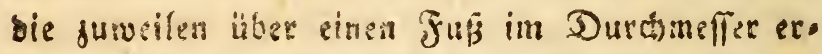
rsictet. In der Unterfsite Der 3rueige bängen erwaz fothtta, blapfarbige 1 bis 3 Sinien lan. g: Jifrimfäbsen herab, welche meiffentbeils gami einfach Inn. Ezegen die Exrremitäten bin werben bite fräbon ojer Gradolla intmer län. ger und bäufiger. 2lut find fis bie: meiffentheile äfig oouc fonit auf vielerlen 2Irt julammen vers warhfen. Gie fithen hier rund um bie Bameige herum, biegen fing aber bennow in artiger Eteio lung naú) sbowärtö, und formiren fehre reisendes 


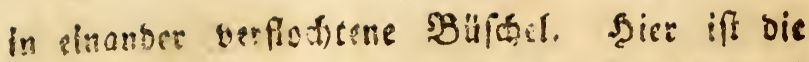
garte ber Scoweln etrab metre gefärsigt, und fowoly sicie, als bre 3 veige feloft weroen gelb, und beflo lebtiofer, is beffic dis Entroictung Des Stamame von Gatter gegangin.

Sjot Difien Es momme hat noth ein ano berer (ser Biegenbart, Hydnum abietinum P.)

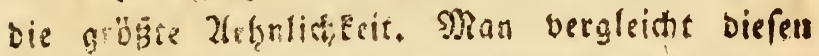
in feirer Gugend mit bem fogenamnten Fauli ober Blumenfoht (Brassicd oleracea Botrytis) weif er ganz meifi, unb eben fo voll uno eben fo geftäupelt ift. Ere hat maniger zonaftomofer, if meiftentheils aufredit, und vertheilt fith-oon unten suf in breite, Gemeihfürmige 2lefte, uns ter welden eirige fo flein lino. Dap man in Greifel fiethe, of man fie mehe zu bent IP fäc:n ooer zu den Leften jäfzlen folle. Im zalter wird et uncein blafgelb, und feine fäbsn ober Stacheln mereen niat felten über cinen balben Boll lang. Die an ben 2 effen find etwag aufs rectit, jene bingegen an ben Enden ter 3weige hängen lang und fenErecht wie naffe şaare hers unter. Hebrigens if er bem borigen fo ätznlich, Daf man jweifelt, ob er für eine beionbere 2ure ober nur für eine $\mathfrak{B a r i e t a ̈ t ~ b e z f e l b e n ~ j u ~ h a l t e n ~}$

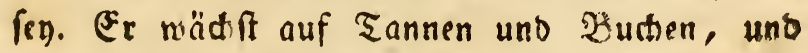
wirb felbft unter ber Erie gefuniben. Die Ex 
242

Theinungeseit von beiben fint bie Monate Sep.

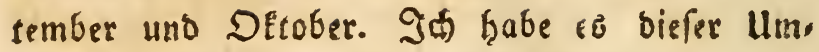
frïnbe halber hier für überflüffig geţalten, ben Blegarbart in biefem Werte Durd 26bileung und Bifonertunft Darzuftellen.

Sie find ubrigens alle bende efone, und merben wie bie Şärentaşsı Eereitet. 



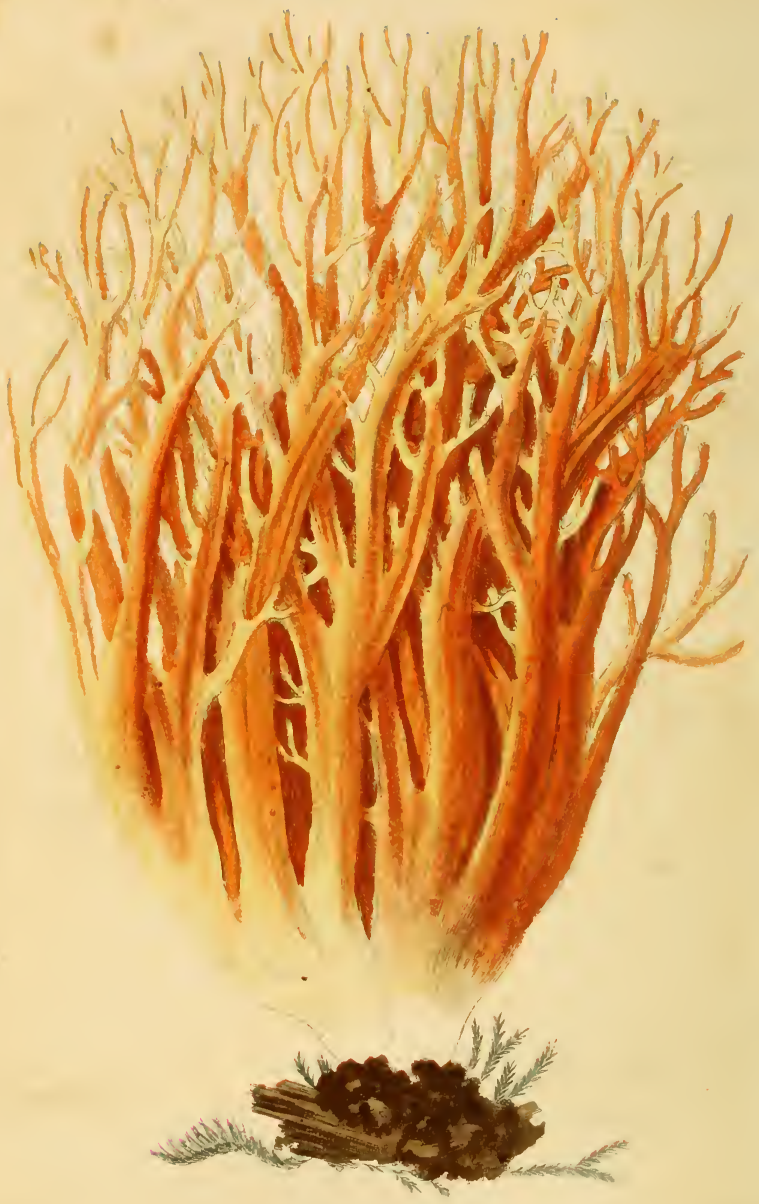

Die qemsene Prörenlaye.

Clažraria fluva Peis. 


\section{Die semetne Sairentade. (Cla-} varia Flava $P$ ).

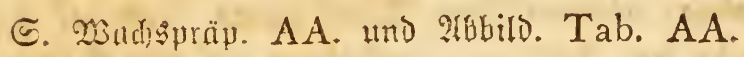

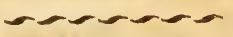

(5) ie beutifiten Benennungen ber (Serwächle finb trobs aller dieffälligen 2ermirtelungen ber Bes lebrten ood) immerbin faranfend, froeybeutig und verführerift. Sie weroen ce auch emig bers bleiben, oder unfere Mutterfpradie mífite nur, ich meif nidit Durd mas für cin Estchal, die allein herrftiende bes ganjen Erobodens mers ben. Mand PI flanze whirbe vielleidit fünfig und

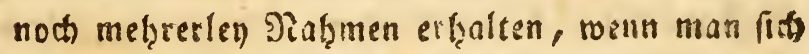
recht eigens bafür vermemben roclle, fie aufjus fammeln und aller Orten ju erforfhen. Zllein

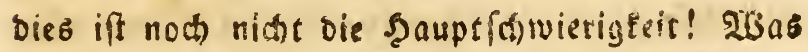
Diefe I ab̨men am meiffen untauglich macht, allges mein aufgenohmen zu merben, ift ber vage Mig̈.

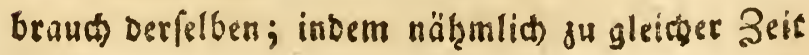


eine unb bicjelte Benennung verfdiebenen, in ofo ters im häd)ften Brabe heterogenen Serräd) burd) Denfelben zu Theil geworben. So heißßt z. (E. bie gegenwärtige Safwammart unter anbern aud 3odfebart: allein nidit genub, bafi man auch now methe anbere Zrten von Clavaria to benennet, felbft einige Irten bon Hydnum, wie wir sereits ben Dem vorlergethenden Irtifel Lit. Z. gefefen haben, nethenen if̧ren Sheil baran. Zlus ber Fa milie ber flectiten, befonbers in ber Sattung Usnea, Cornicularia, Stereocaulon etc. rürben wir leidt ein Daar Duzeno Zurten zufammen bringen, bie fo genannt werben, unb um bie Ber, roirrung von Babnlon vollemmen berzuftellen; fo finden wir noch ein ganjes seer vegetabilifwer Boctabärte in ben Familien ber Jyh̨önogamen, bes fonbers in ben Ënttungen Spirea, Eriophorum, Tragopogon, Anemone, Aira, Festuca, Di-

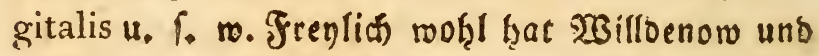
mentr andere ein Sittel verfudt, bie Eintieit ber beutichen Benennungen herzuftellen, indem fie bie

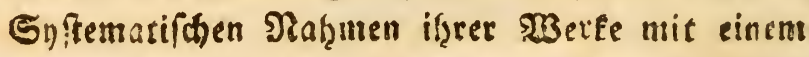
Bartungs, uno Tribialnaf̧men ins Deutfhe übers

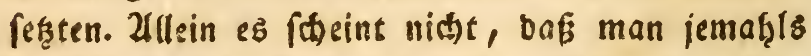

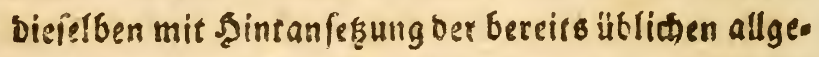

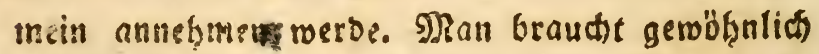
bie beutf the $\mathcal{B}$ enennung nur baju, umim Baterlante you don lingelefgeten verfanden ju warben: und Da 
soirb bann bet Bauer uno ber Sdrwammlräm. mer, ber fein rebetag Eeine Species plantarum in bis sand nimms, faton fortan ben Den Sris vialnalgmen feiner Seimath verbleiben, ohne fid uı bas nomen genericum zu befiimmern. Heber. bies gerathen auth viale biefer liberleşten Naḩ. men fo fonoerbar, daff fie ifrer Deutfofecit un geadter nict) um ben minbeften Theil berftänb. licher fino, als wenn man ben lateiniftien $\mathfrak{R a f}=$ men unberänbert gelaffen ţätte. Şat man ood fo vielen Taufent anbern auslänbifa) शnalsmen bas Bürgerreकt vergönnet: trarum nicht aut Denen Der פaturprobutte, da biefe ben Borgug bor allen übrigen haben, mit ber g:ößjten गुe"̌̆ rifion aller Orten uno bon allen Nationen ver. fanben zu werben? Doer idmeidtelt es vielleidis Dem Reidthum unferer Mutierfpracte, wenn

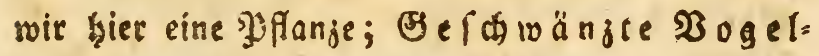
mild, cine anoere Ropfförmiger $\mathfrak{R}$ ats terE opf utb wieber eine anbere Exiedender Froftlöffel nemmen? Eher wollte ich es nod hingeb̨en laffen: geftoränztes Ornitho: galum, Ropfförmigez Echium Erie. d)endes Alisma zu lagen! Ullein, wenn wir fhon ben lateinifhen Battungenabmen ais. neţmen, fo wirb waţrlic Der Trivialnabme auch nitht ment to viele Beidtrerlid) Feit haben, um bem Sebädeniffe aufgebürbet rostien ju tön. 
nen, zumafst ba getwiffe Trisinlmakmen in fo vielen Battungen vortommen, onf fie aud bem fowerfen Ropfe enolich geläufig merben müffer, mie bie 2hesoulufe: officinale, vulgare, Europaeum, vernum, aestivum, autumnale, sempervirens, semperforens, giganteum, nanum, multicaule, cernum, dichotomum, rectum, repens, volubile, hirtum, lanatum, acaule, alpinum, montanum, sylvaticum, arvense, palustre, spicatum, paniculatum, multiflcrum, lineare, ovatum u. ๖. gl.

Der Efimamm, won bem bize bie fiebe

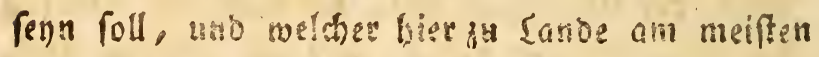
unter Dem Sahmen: Bä ärentaze befaunt iff,

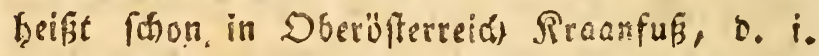
Rräbenfü uno SalnnenEanm, fo wir un ?Re genढ़burg Sennenfamp, in Etemermarft: $\mathfrak{B} \ddot{a}=$ renpraţe, im eoleren Styl $\mathfrak{R}$ ärenpfote: in Sadt= Fat 3iegenbart; am Sarz Betskart, Bockbbart;

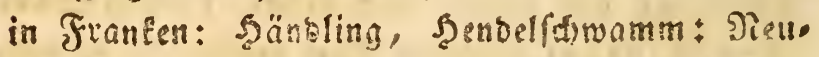
mase in ber Oberpforg: Shirfardinamin; bey

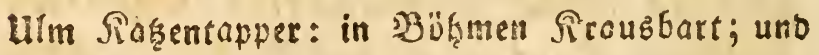
nod) über bies kie uns da Eorallenfatmanm,

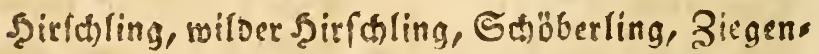
Gärtgen; auf Şolläntif Koraalachtige Knodszwam, Handkampernoeljes, Vingerkamperneljes, Geitenbaard: Dänifch. Koralsvamp. Schwed. Koralklubban: Engl. The coral cla- 
varia; Frang. Clavaire Coralloide, Gallinole; Barbe de ch rre, Mienottes, gantellines, barbede-bouc, bonquinbarbe, tripette, Chereline, pied-de-cog in Der Zausmipracts: Dzenellie: Span. Munerillas, 1 mai. Kuratsa, Kozi-brada u. $\{$. w.

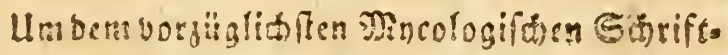
fiefler, en Dr. Merfoca zu folgen, nenne ith Diefen Esmamm Clavaria flava, obgleich er Lubrigens in ven meiften floren unter oer Benene nung Clav. Coralloides vorf̈ommt, unter welo tor jeoob mchrere vorhin nicte hiulänglich beob.

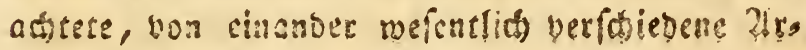
ten bigriffen wurben.

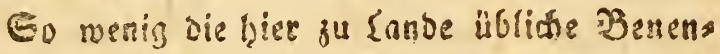
nung diefes Gomammes an ctwas Särtitheb ju erinnern geeignet int: fo wirb man mir bennod willig Jenfallew, wenm in befanpte, bafs ex un ter bie artigiten, nieoliofinan und liebensmituigs flen פaturprodufte gejoblet zu rerben verbiene.

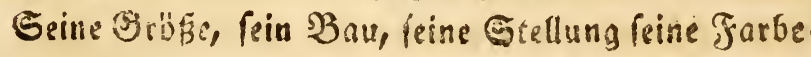
alle iff in bie befte Syarmonie vercinigt, um the auf

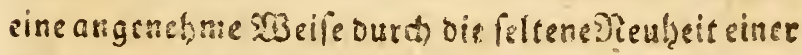

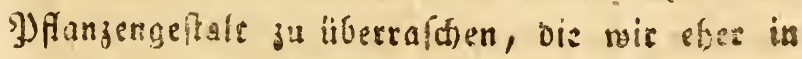
oer Fanitie oer Eorallen oser an geroiffen sRines ralförperu gefuct) bätten, als in bem sibietise De: Flora. Mandmalgl follte man fuff glastst,

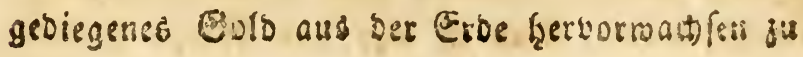


148

felyen, befonoers, roent er in ratwarzel Moors Erbe zrwifhen \{aubmooren und If fällen heraus. brint im Dideidfe bes Butsentaines, wo keine anbern Bewähfe, ale nur jene ber heteroboxen Familien der Eryptogamen geopihen. Sheint es boch, ba官 uns Mutter Patur in biefen Son. Derlingen lauter Einnbilber und geţeime $59 \mathrm{M}(\mathrm{y})$ frerien habe mittheilen wollen! 200 weip aud wie reidlid fie uns now bie Shublse lolsnen wers be, mana mir einft in biefem Stubium igrer Beiohenfurad) grübere Fortfaritte gemadit und eine gerwife reelle Fertigfeit erlangt haben mers ben? I由) mainer feitz finte bie Biloung biefez Sđ) wammes zu intereffant, als ba Ioeenfolge ban feiner Betrawing in meiner Seele

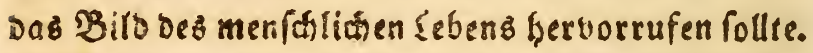

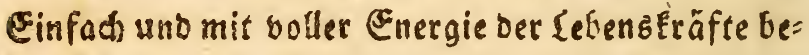
treten wir bie Bühne, balo aber fuchen frwir

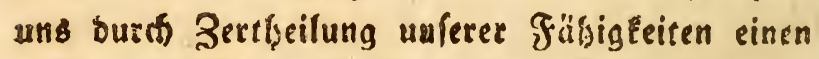
gröBeren Wirtungefreia zu erringen; und jese biefer Fähigkeiten erweitert fid burch ncue fort. gefeste Entwicklung. zaber insem mir auf tiefe 20Beife unz inmer meffe unb melse im limfange exweitern, fo entrerben mir zugleid unfer urs fpringlidies SBermögen, uno fielie! mit einem Mable find fie an ihrem Ende bie verwickelten Zmeige unferer Tenbenz nach fremben Berüh

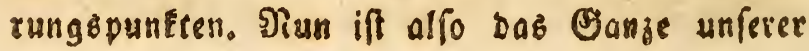




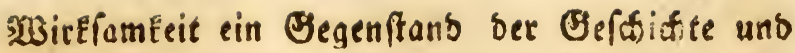
ber Beurtbeilung! Bollftänbig entwickelt, soie zah/reich fino nidit bie feinern Rüancen unferer 2luzgeburten. Dod tragen fie alle bas Bepcäge unferes Uriprunges und nie bürfen fie bas SRaß unferer orginăllen Znnlage überfकreiten!. Wुenis ger Entroidlung - weniger Berluft bee Siräf. se! Das Leben eines meníden ift ein indivis suctles Sanze; ez Eann Modifitazionen, abee Eeinen 266rub erleiben! Der Trieb zur Ents wieflung ift ein 2 sere ber פlatur, bie Entwick lang felbft bängt von ben Umftänden ab.

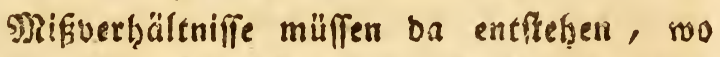
geroiffe einzelne Trähigkeiten auf Roften ber übrio

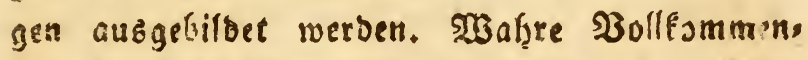
beit fest ein Ebenman afler Thille bes Sans gen vorats. Darum fino Dienithen voa vielar

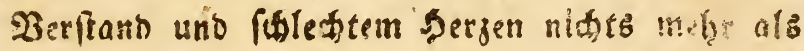

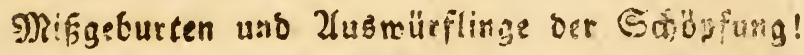
unb ein rotigefialtes 2 egetabit is von einem

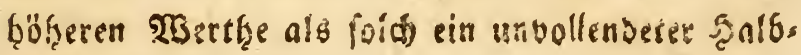
menfth!

Die fyorbe ber Somämme wath guar fron

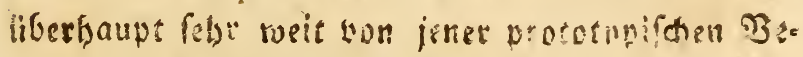
getaticusform aú, bie wir in oe" büdifen bes

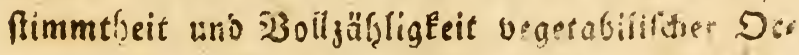
gane, rvie d. $\mathfrak{B}$. in ber Familte der Relenge

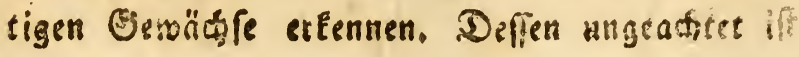


Doth bie Frenheit ber Patur, mit melder fie Dis verfofisbenen Gattungen ber Stwänme ento worfen, nod fo uneingefobränt, dafi fie untes= einanoer felbft eille nich minoer teträdotlithe Derfobiedenlazit barffellen. Eine Amanita uno ein Jgelichmamm - weld ein ungetgeurer 266 . fano! llno bennod blcibt ifse für bie meiftens fefgr zahlreichen 2lrten zut Zuffeflung verfotes bener Biloungeformen roch ein reiter, und wie es fheint, faft grängenlofer Spislraum. Ja feloft in einer uตb ter näbmlithen 2lrt bemeifst fie uns not Die Unerfकüpflitfeit Deb Erfinoungsgeifres, welcher ben grofien Sylan bes Weltalle entror. fen uno bis auf die leşten minutiellen Details entfaltet hat. Die झannigfaltigteit dec Epizlar. teit unb doänberungen ift $\}$. (5. ben ber gegen. rärtigen Stroammart in $\mathfrak{W a b ̧ r b e i t ~ s i n ~ S e g e n . ~}$ ftand ber Bewunderung. „So grof auch immer, fagt Holmskiold: *) bie Berfdiebenfreit Der abropichungen in oer Billoung uns $5: 0 ̈ \beta 3$ Der Sictorallen bematfe mirs; fo if fie oennoth in ben insiviouellen Muftern biefer affidmammes nitht minber erheblith; und unter Taufend Erem.

F S. Defien Beata ruris olia : Havniae 2799 fol. p. 114. Tom. I. 
plaren mith man Eaum suen auffiaben, die

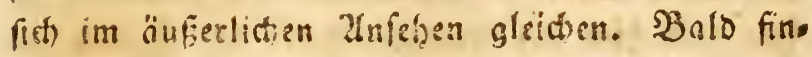
Den wir frinen EtrunE geffrectet, aufredt, breit uno frach gedrücti; ein amoeres Mahl iagegen Eurg, Darniedergebogen, dick uno runo. Der eine

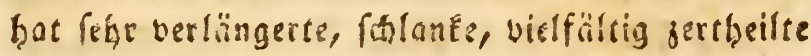
Zefe, ber andere absu Eurze, bicfe und einfadis. Ein gleidhes Bervantnif hat es mit ber Eröß̈e und mit ber frarbs: derns einige erfeben fich fu einer söbe bon zețen Bollen, wö̈reno bak an. Dere Eaum die Sälfte diefer Erößse erreichen. Ibre (3:undfa:be if meiftentheils reib̆lia), bot fallen andere wieder melir in Die Farte ces Plurfur und Des Boldes. Ëinige mentige findet man aud von

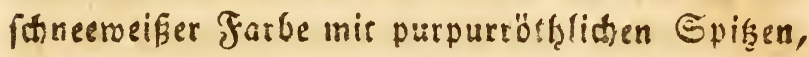
anbere geltlich und Eunt geffreift oser mit Yur. purfarben Flecten bezsichnet. Jugkefondere pran. gen die jugenolichen Inoivituen nit ben lethafte.

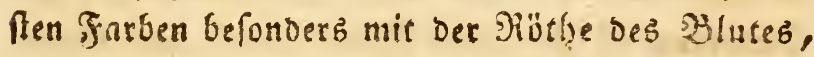
Doer mit ber blendenben Siothe Der Teuerflammen: u. F. w."

2utsings entfernt fif bie Etruftur ber

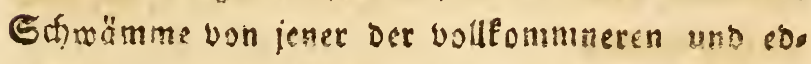
leren syfarzenfamilien fo fetre bos mas brn eio ner füdrigen lleberfict Diefer Naturgebsipfs faft Linftano nefimen follte, fie in bas fervöbercith aufjurefomen: allein, auser ozm, sof fis miete!f

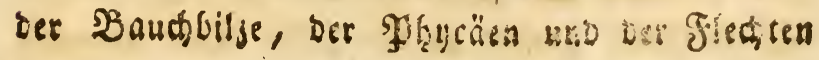


mit jenen wieber in Bufammenfiang fortmen; fo hat nuth hier bie bilberibe Sand ber פlatur ben näl̨mliden Eseift ber Ifnalogie entroickelt, relchen wir to oft bey ber genaueren Betradtung iffrer YorobuEte zu betwunbern Belegenheit uns Serane laflung finben. In einjelnen Irten ber Eđ̆räm: me finden fith nod immer gewiffe Spuren ber prototypifaen formen: nur find biefe bier ifolirt und nur felten treffen 2 ober 3 in einer und ber= relben Biloung sufammen. So finben wir in bem

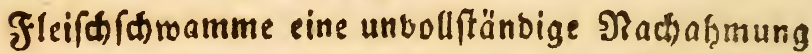
von bem Blumenbaue ber Dorstenia uno noch mel̨r ber Cynomorium und ber Balanophor.. So gleistet bie Trüffel ben Rnollen ber Jalappa. So erinnert uns ber Eorallenifhramm an bie F̈bren, fo ber Epatelidiramin (Spathularia flavida) an gewiffe Tangarten ober an bas Blatt ber Sarracenia flava uno bie f̧ier vorgeffellte Bärentaje iff überf̧aupt Strauchartig, uno fönnte allenfalls mit Anthyllis Erinacea, Salicornia Caspia, Statice reticulata, Anastatica Hierochuntica, Baeomyces rangiferinus u. f, w. ver gliden werben.

Der Strune diefes Sdrotmmes, melder meiftens nod unter bar Erbe ober in bem locfern Dünger ber verfaulten saubmoofe, unb abfälle von Bäumen uno Rräutern verffecfe iff, rid)tet fic meiffens gerabe in bie söfte. Ssin Umfang 


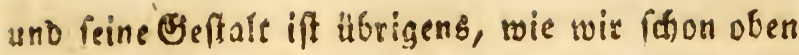
in ber aus Solmefiolo überfeţten Stelle gefefren

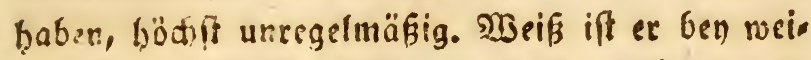
tem in sen meiften fällen, menigftens fo weit ex in ser Eros verborgen fiest, uno ber llebergang in bire Farbe ber 3meig: ift ganj unmerflid). Seis ne Eucoratebene (im Durdictinitt) ift jenet ber ers ften Bertheilung, fo roie jebes Mabl bie eines 3reiges jesser ber \$eräflung und baber bie er, frere aut orm Sehalte aller Ertremitäcen jufams mengenofimen gleid. Rönute man Dzn ganjen Sthromm mit Bermeioung aller Swiftienräume zúammen fomelien; fo witroe man alfo einen regelmäbigen Eylinoer erfralten. Die Uefte fino balo metgr balo reniger in einanber berfoctiten; mifitens flad gebrudt uno der \{änge nach, f(t)rach gefurdit. Die Bertheilung felbft hat gar - Eeine Sefetse. Manchmal wahfen bie und on cis nige 3reige ourch sab Berühren zufammen. Die Extremitäten, weithe ing gefammt eine gan glei. de ग-oportion ber Diffeoarfiten, fins and an als len 3reigen von gleicher Beftaffenbeit, fie thes

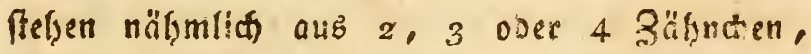
welde gleidjom roie Snofpen sines Bemädifeo ausfeben, unb allezeit sine röthlithe farbe has ben, ber Sdinamin mag nun fhon golbaelb wie bier, oder weis, fleifhfarben, braun, röth. lich ober wie immer gefärbt fenn. Der fenérect). 
te Durhifuitt entoeft uns ein feires, weifsz, sichtes Fleifh, wobon forohl ber Sauprfamm als auth alle feime Beräfllungen voll fino. Die blithano: Farbe feiner Sugend geht mit ocm Ulter in die Trauerfarbe übar $o$. $i$, in eire fhmuşige Erofarbe, bergleicien wir \&. E. an ben BI Uutien der Hesperis tristis, Hyacinthus Muscari, Pelargonium triste, Verbascum ferrugineum, Orobanche major, Epipactis Nidus avis, Silene gigantea, Cyperus longus u. o. g! bemerEen.

Der Gtantort biefes Reulenfarmammes find bie aus Budbr, Eichen, Serchenbäumen u. o. gl.

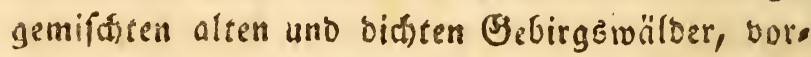
ditglich bie ber finlEgebirge, in beren reidhen Enaten bie Saorbe ber Saubmoofe ben fatwars zen Boben mit nieblidjen grünen Teppiaen und bequemen Sुolfern ju tapejieren geroohut ift.

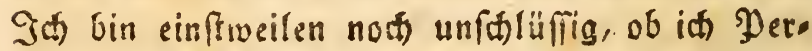
foonz Clavaria formosa mit jener für einerlen 2irt, ober für eine eigene balten folle. Sie lebt bay ure in oen llmgebungen ber alpen, und wirb im Spraude gar nicht unterfficben. Banz Europa ift bas 2 aterlano Der Clavaria flava. Man findet lie in ben fïblimeren Behirgen foton früfer, nämlioh im Junius, in Dell פorolän. bern foume fie erft im 2luguft und Siptember , zum Borfobrin. Sie räd) fefar fotnell, uno 
Solmekiolo maldet, er habe nach 9 Sagen, als

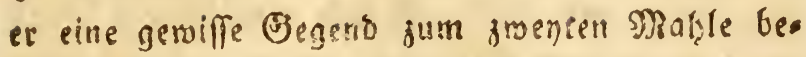
fuchte, in melcher er das erfte Mabl keine Spur Daton gewahe wurbe, eine grofie Menge ruton ganz entwickelter Bärentagen gefunden, welte

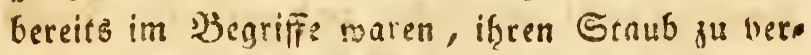
freuen, ienes faine Dulber, dab allenthalben aus De: ganjen Dberfäne ibrer Zreige hervor quillt, und meldes man 10 , mie an ben ütrigen $\mathrm{Sth}_{\text {wäm }}$ men fïr eine 2lrt son Saamen ereläret, moburdh fith bie 2rren ber Stroümmz anbauen und forts pfianzen.

Man pfrot viefan Strwamm aller Drten bäufig zu verfpeifen, und es ift im eigentlidfften

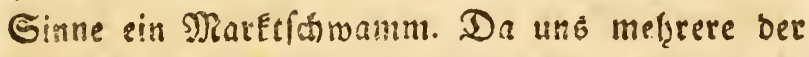

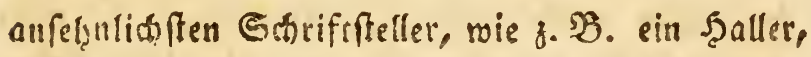
Bitlliaro, Solmefiols u. b. gl. für faine lln.

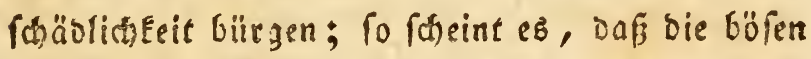
Bufälle, welde angeblin nath Souttunn auf reis nen Esenus erfolgt fenn follen, und wovon auti id bie und ba auf bem \{ande $\mathfrak{R a t h r i t h e n ~ e i n g e s ~}$

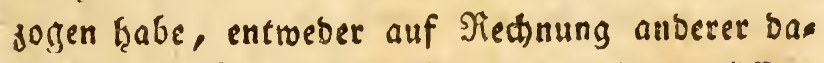
mit verwedffelter 2 frten bezohen werben müffen, Doer baj vielleidft verborbene mit Infef́tenlarbent bebölEerte Stücke aufgenofimen rurben, beren Benus frenlid) wohl ungefund und mit Ractive. hen vergefellfwaftet fenn muf. Iक rathe batier 1.) genau in 2ldte zu nef̧men, oaś man ja nur 
$15^{\circ}$

ädte Bärentagell aufreţnien inögr, 2.) oafi man nur junge uno frifche Stücfe erroähle; 3 ) oaf man fie vor bem $\mathbb{S}_{e}$ braudio waithe uno bon allen

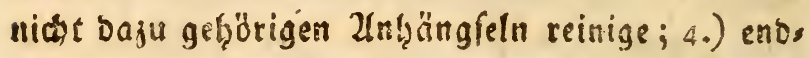

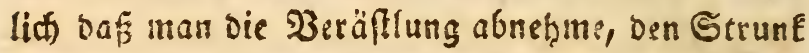
inwenbig mohl unterfuche, unb wenn fein Fleifh intentig anders, ale weif ift, ifnn lieber hinnege werfe, als fith ber Befahr ausleţe, Bauchgrim, men unb Erorectien zu beftelen.

Man ifft diefan Sthwamm als Sallat in Bermifáung mit Bswärgen, Tifhen, Rräutern u. o. gl. nachoem er vorier mit beiffm 23 affer abgzorübet worden. In Franfreich pfleat man ihn meifteng frifafirt, o. i. Elsin gethack mit" Şühnerfeift in Der weifgen Eatte fu verifei. fen. Man inacht auch Röbthen navon, hi man wie bie vom aehadfen Fleich gemathen Frica = Dellen verfperf. Dir beffe Bereitung olt rarin hefte hen, wenn man bas gut atgerwaidserefficif bies

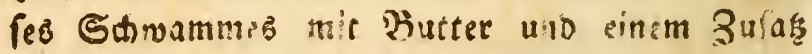
bon Salt, Seterfilistitraut, Majoran 1. 5. al.

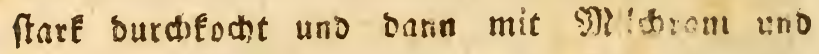
Energelb noth etmas eintoht.

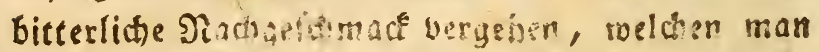

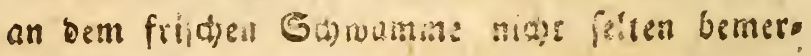
ten mag. 



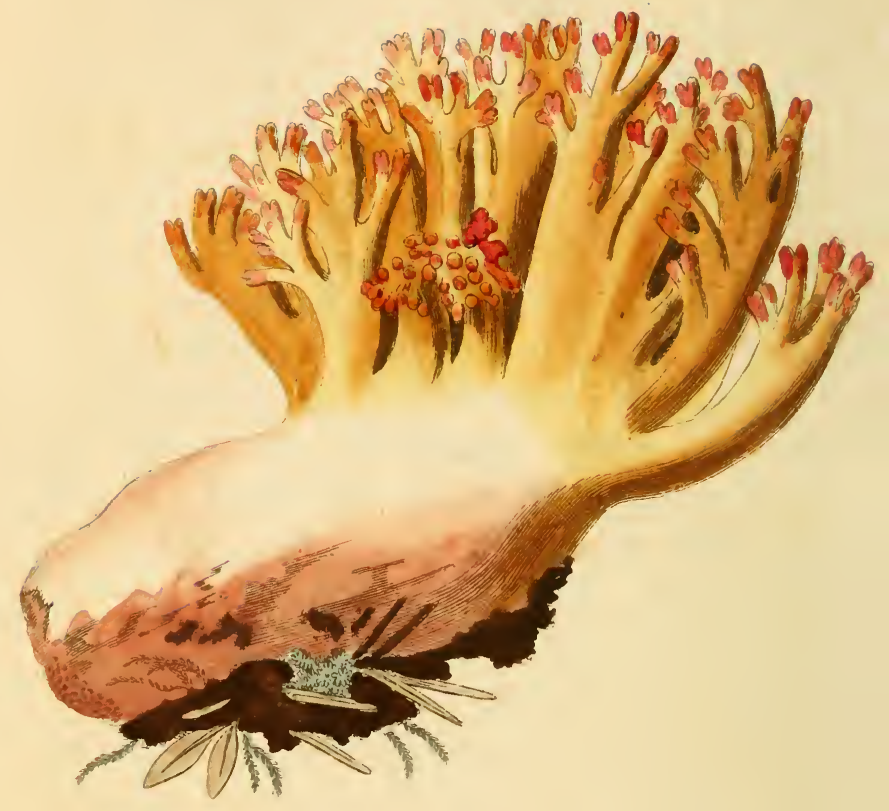

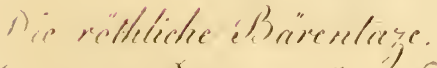

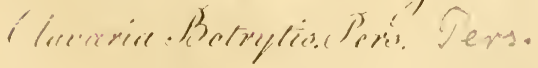


XXVI. Die rơthltấ ふ̧årentage. (Clavaria Botrytis. P.)

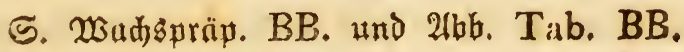

9)

Ran Eann biefen Sthiwamm nut burf foldere Mertemahle von bem vorigen unterfaciben, wels We zwar an fich auffallent genug, in ber That aber blof zufällig und von bem Ëinflü ber 2 Bit, terung, Des Stanbortes und ber Jaf̧reszeit ab. bängig finb. Ith fand ifin oft in ben Difterreis wifthen alpengegenoen, uno id getraue mir nidit, if̨n für etras mef̧r, als eine blope abart bes vorigen zu erflären. Er mirb von bemlelben blof Daburch unter/fieben, Daß er barniebrr'iegend, zäher, innerlid zum Theil von feiner Färbung Dur(t)brungell, uno mit einem fehte engen Sebrän. ge biffer Hefte geterönt ift, bie fich entroeder un. mittelbar oder both nur bermittelft fefir Rurger uno zarter $\mathfrak{B e c a ̈ f f e l u n g e n ~ i n ~ r o t h e ~ o b e r ~ g e l b e ~ f t u m p f e , ~}$ bfters feţr zufammengeţäafte 3adthnden endigen. 


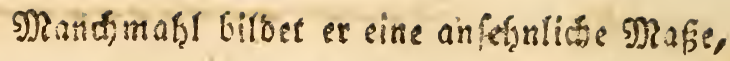
wohl von anderthalb Fuf im lliniange. Ein an. Der Mathl fingegen ift sas ganje Bezetabil faum

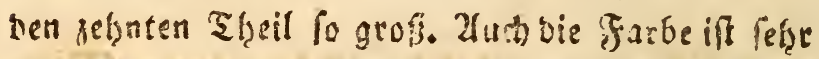
veränderlich, wik ben dem vorigan ifff(t)ramme. Daf̈ es nitht immer ein 3roerg fen, fonsern ben feudtar 2 Bitterung und in eimer forttigen lage manctmahl in fafre lange und vilfabe Baräfe, lungen aubwathe, betratsptet fefte jerfoon. Sier

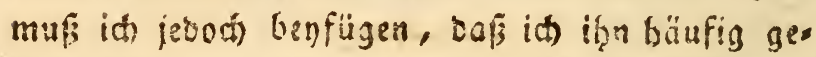
nug mit dem gemeinen gelben 50̈.nerfomamme in Befellidint nuf einerlen Gtandorte unb in ber

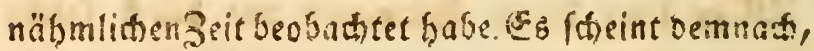
onß noth manderlet anozre llmände riefe rbän.

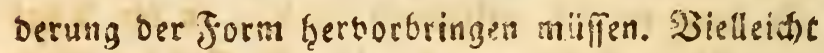
geht ez bier eben fo ju, reis ben bain oben uns ter Lit. T. ermäbnten siabafon (Boletus poly= cephalus) uns er Enrn vor llebermaß́ Der Dyro= lifiéajionsanfänge nidht zu feiner Bollenbung ges langen. So entmannt fribjeitiger Sejolectibs. trieb aut bic animaifínen Inciviousu; fo ers ficter ber überfpannte Eifer maitieg elgracigigen

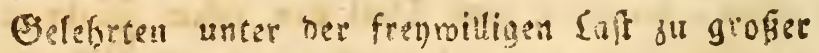
und gu zahtreither Unternefimungen.

Stanbore, Gabrzjeit us) Melraud fint mit bem bosigen, fo wie Seftalt uno Färbung ciner.

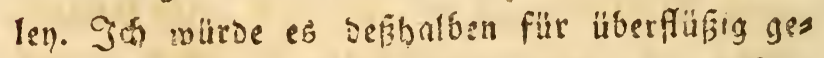
halten haban, dielen Sofromm in einembefons 
beren Zlrtilel abjuf̧andeln, menn nid)t bas $2 \mathrm{~m}$. fetgen fo vieler 2lutijoren bie ifgn für eine eigene 2lrt erflären, mid biefu gleid)fam gejmungen bätte. Uno in ber That, bie llnterfheibung bies

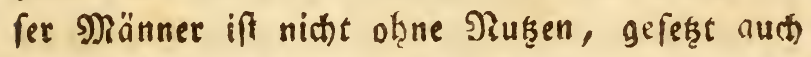
Daßj bie angegebenen Unterítecioungemertimable

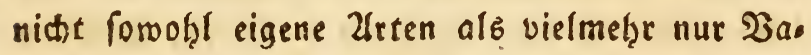

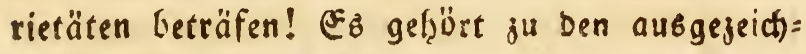
netften Merfwürbigteiten ber Strwämme, DaF in iheer Şorbe auth bie geringeren unftäten Mos bifiézionen auf eine fehe wefentlictie Berfaties Dentheit ber innerlichen Eigenfidaft reuten, uno Dafis es in einer und ber näfonlidien 2lrt efsbare und giftige Safrämme gibt, bie fit aber bens now Durh gemiffe habituelle Rennzeiten mie

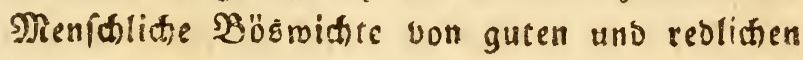
Seuten in ber $\mathfrak{g h y f l o g n o m i e}$ uncerfheiden *). Es wäre baher feh̨r zu wünfthen, daf man ges

") (E) gibt jesob and in ter Fanilien ber Pobriogas

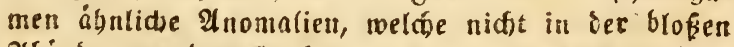
Abánerung ber Sarbe, Bildung n. F. w. fondern and in einer beftimmten 3erf́biebenbeit Der inner: lidben Cigenfduaften 3. 3. Des Beftimactes, ber Entrituflutrg, Der Dauer u. D. gl. beftefent. Die

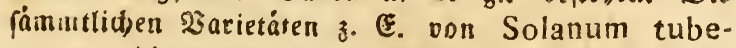

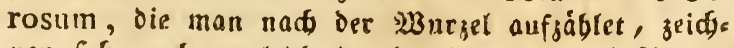

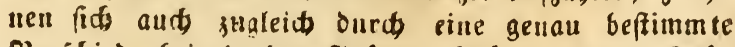

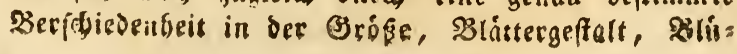


nau bey vorfallenden Boglegenfeiten unterfutien

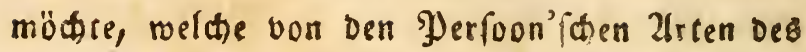
Reulenitwammes, ber erfien zatheilung, bie Solmsfiolo ju einer eigenen $\mathfrak{B a t t u n g ~ ( R a m a r i a ~}$ \$örnerforwamm) erhoben, bie oben erroähnten bebenElichen 3ufälle ju verurfacten pflege. 21. lein bisher ift man bierüber - leiber! noch im Ilngenviffen! Für bir unfäblichffen und am mindeften gefährtiben balte ich einftreilen bie benoen hier aufgeftellten Şörnerfónömme, weil Dor allyemeine Ssebraud aller Pationen feit Gabrfunderten ithre llnfalulo vertheibigt: nit mönte Jerfoong Clavaria formosa biefen zu= nämif ben mindeften Berbacht verbienen, weil fie befonders ber erften bon den birs vorgeftelle ten Zrten fo äbnlich ift, das fie fich fait ein.

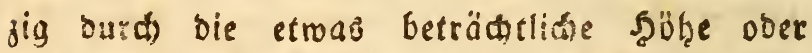
Swlanfleit iffer lefte unterfdeibet, uns baher gercoe das Eegentheil von Der 2 rt bes gegen. wärtigen ZlrtiÉls darfellet.

thezeit, Shlumenforte 11. f. w. aus, fo bos man jeve Eorte ans dem Bewédice fith erfenuen und unterfectioen fanr. Eben bies gilt and voll Vitis vinifera, Fragaria vesca, Pisum sativum, $\mathrm{Py}_{\mathrm{y}}$ rus communis, Juglans regia, Brassica oleracea, Ficus Carica, Dianthus Cariophyllus, Primula Auricula, Cucurbita Yepo w. f. m. 

C Die stockmorechel.

-Habella esculenta. Pers. 


\section{Die Stodimordiel. Helvella esculenta. P.}

ธ. Wsudspräp. CC. und abbilo. Tab. CC.

So merentitroig alld immer ber Ulebergang fenn mag, welden wir in bem gelben ફ̧örnets formamme ober ber Bärentaje von ben feörners (đ)rämmen zu Den Stastelfdrömmen beobad. ten: fo ift bod jener von den Faltenfichuäm. men ju ben Mordieln mittelfi ber Stocthord,el noth viel auffallender und einleudstender. Bers gleidien wir fie endich mit ber Baffarbmordel, ober mit Yentenats Morchella Tremelloides: fo follte man fin faft geneigt füfllen, oiefe bende Sattungen in eine einzige jufammen zu formel. jen unto bie 2rten voll beyben in ununterbroches ner Reif̧e aufeinanber folgen zu laffen.

Tुa habe mid bief̧er von bem Dafeinn ber eigentlichen Helvella mitra in Defterreich noch nimt ïberseugt, uns übergetse fie baf̧er bierorts mit Stillfwreigen: follte fie fich jebow irgenomd vorfinden; fo Eann fie ofine Bebenfen unter Borausfeţung ber allgemeinen Boricthtzregelu fo gut, wie alle bie übrigen geffielten 2trten von Helvella; die man im Deutforn ofne Unters

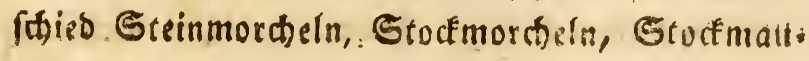




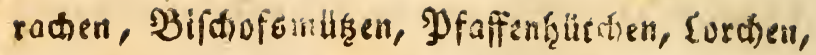

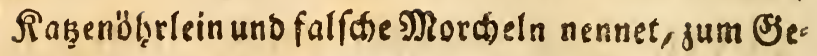
nuß̧e angewendet werben, und es wärbe von if̧nen allez dab gelten, was id hiervon ber vo:liegen= Den bunkelbraunen Stockmordel zu fagen habe.

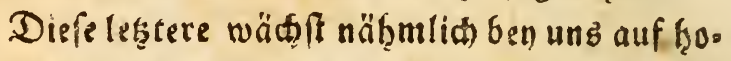
hen Bergwiefen, $\mathfrak{j}$. B. in ber Eegeno von silienfelo - fömmt alloort im $\mathfrak{M a y ~ z u m ~} \mathfrak{B}$ orfwein uno wiro efen fo wie bie gemeine Morchel zubereitet uno ge=

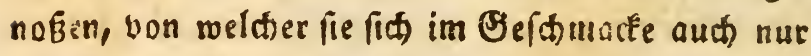
feţr renig unterftheibet.

Diefe unfere Stockmordiel, weldie audh am Sarb und in B̧anern zu Şaufe iff, bat einen glat. ten (Furdienlofen) unterha!b etwas Rnollichten un= etenen, ungefähr 1 Boll bicfen uno 2 Boll hohen, inn= werbia Zellenartig auछgeţ̈blten reeiffen mit bem feinen Fruchtpulver graubräunlich bereiften StrunE, auf beffen ftumpfer Spitze ein oberb̧alb fow war forau= ner unterhalb weißlicter scut feft fiţet, melther, nicht fehre tief in metgrere jiemlidh grofe, wollen:

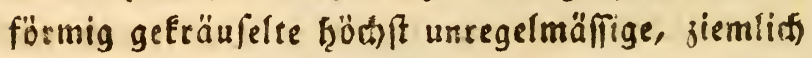
ausgebreitete \{appen zertbeilt ifs, wel bise auf if̧rer Dberfähe mittelft iffrer, jeood nur feichien uno frumpf geraltoten faft Rreisförmig georbneten Bellen mit ber gemeinen Mordiel viele 2leţnlith. feit faben und gu ibe eine fetie nabe Bermanoto fhaft Eerweifen. Diefe Lappen fino jiemlich bict und yon einer faftigen Trondsähnliden Subftand. 



\section{XXVIII, Die Serbitmurchel. Helvella Leucophaea. P.}

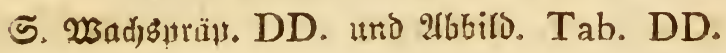

$\Re$

od eine Urt bon Faltenfarwamm, die zrat bey uns nidht in Menge vorfinoig, bie aber als lerbinge effor ift und baben now bas ßorgügs liche an fith hat, im foäten Sarbfte zu exfdeis nen, too wir nicht gerrotent fino, Mordieln uns Mortbelartige Sdwnämme zu verfpeifen.

Perfoon, reltbem meine 266̧andlung bie. fes Shrammes in bem größeren Sdwammiverte (Drfterreido Sdwïmme T. I. p. 197) ju Bsefidte gefommen, lyat fich bierüber in einem Bricfe geäulfert, bof ex ungewif fen, 06 bie bort vorgeftellt Serbftmord)el nicht vielmehre eis ne sigene neue drt a:trmade. Allein, ba id bie Urfathe entoefft habe, welde it̨n zu biefer 
164

Dexnuthung verfüfret hat; fo liegt 8 inir $o b$, biefe Sache aufjuflären, und bie 2 Bahrheit in ciir beller rīint gu Gringen.

Da ber Berr Berleger Der Defterreichiften

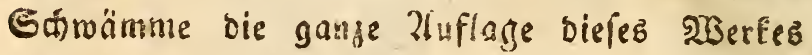
belorgt; fo gufbals cb, oafs cinige junge reute ifm iluminirte Tafrin ubarbradten, mit bem 3orgeben, id bätte fie fobon burctigelehen uno gut gebrefen. Da nun aber diefes 2 orgeben falla war, uns fer. Oseiftinger, ber if̧m feinen Blau= ben (t)ente, Dobutch bintergangen rutbe; fo geforoh es, dafi sinige eremplare in ber Sullu. mination nidft ganz rictitig ausfielen; und ids

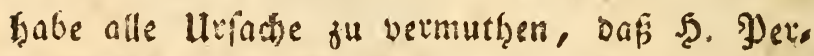
foon eben ein foldes Exemplat exhafien habe.

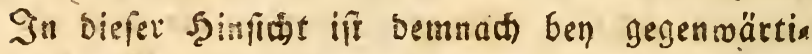
get 2ten Borftellung um fo viel mef̧t Fleifí und Biţuthfamét angeroendet rotben, und

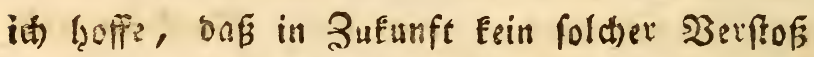
fint wieder eleignen wexbe: Denn rass bie gllus mination betrifft, fo überniţmt Sr. Betleget meiter' Exine Exentalate, alo foldhe, morüber

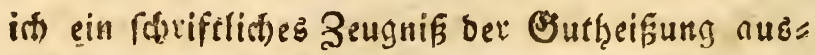

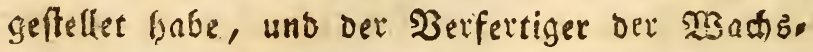

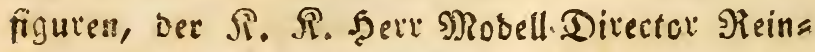

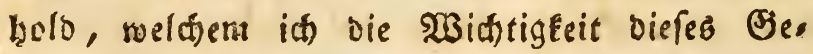

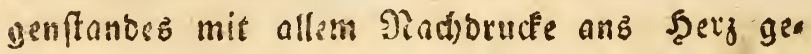
fegt babe, madit fich folbft füL die rictigtet 
Der gefertigten Exemplate verantwortlid, aufser: bem, bof ex mix rie beiliglte 3ufage getban, Erin Stück abjuliefern, roeld)es ich nitht angepes hen uns gutgebeisen bätte, und bnher aud alle unn mir gerügten Unvollfommenbeiten mit bet pünÉlichften (Senauigfe:t ju berichtigen. Uno fo hoffe ich Denn für die Zutunft das Beffe!

Uim nun wieder ju unferer \$erbfimotchel fus ribf zu ecmmer, fo fann ich betheuern, oafi ich biefen Sd) ramm mit aller bet nötbigen Sorgfalt gaprüft und forohl mit den Befdreibungen, als mit ben 2lbbiloungen bet in meinem gröfseren Sकrammrerfe citirten Sकriftfteller nohmentlid mit Battarra, Bulliard, Schaeffer, Schrader, Scopoli uno Sowerby genau verglian habe. Insbefondere finde ich bie zrbbiloung bes Sowerby fetre anpaffend und aller linterfated zrois fhen biefer uno unferem Sthwamme befiünbe föttfens in ber etrong färferen Färbung deb Sutes uno in ben etwas tieferen und sableiches ven গiungeln. Zllein bergleithen Dinge änbern nath

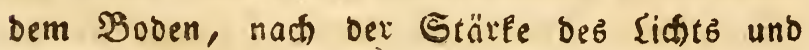
nach ber Berfhicoenbeit ber 2 Bitterung zu leicht $a b$, und bie gemeldeten Ilnterfaiebe find iberbies

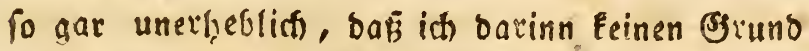
finden Fann, unfere Solbftmorhel fiir eine eigere Xrt zu erElären. 
Der Etrune ber Serbftmorchel ift weis, faft Regelförmig uno auf feiner Dberfläche ber \{änge nach mit berablaufenden, gebogenen, theils ans gewachenen, theils frenen Surd Dueerberbinburs gen vereinigten Sehnen bergefralt umgeben, dafi er auf cen erften 2lnblick einem SSitterfarramm (Clathrus) natjuarten forinen binfte. Seine in. rerliche Subftang ift bon gand gleider B̉efhaf. fenheit. Die herablaufenden Gef̨nen bilben alfo mittelft ber Quecrberbinbungen ḩäufige Bellen, bon reltien jeboh faum zwo einander gleichen. Durdifdneibet man ben StrunE; fo fiefit man lauter burd)fonnittene Sädfe obel (Sanäle von liers fhiebener Beite und Tiefe.

Der Şut, bet wie bey der Stodfmordel auffişt, aber nialt fo bunfel von Farbe, fonbern

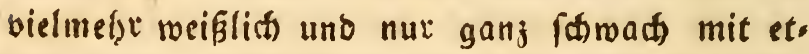
was renigem Selbbraun befhmut ift, hat noch mebr ben Bau der cigentliden Mordeln, als bie übrigen Biffofsmüţenartigen Sđfrämme. Won biefen gleidten einige gemiffermnfen ben Des sisen, ober ßBetherfatwämmen, inbem fie ihre Sappen in bie sobhe zurükf flagen uno wohl gar 3urveilen dem modernen Sønitte ber jef̧t genöhn= fichen Şüte nahe tommen, bie freylich aud Im. pfe!artig genug ausfelpen, uno auf ben galanten Rümpfen unferer Stutzer nid)t weniger grotezE

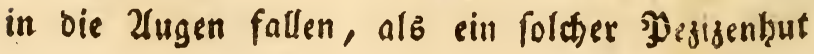


auf bem Stheitel einer Mordiel ober einez Sits terfithammes. Der Şut ber Şerbftmorthel legt fich vielmefre nach abrörts, wie ben ben wahren Mordielr. Dod fino feine Unebertieiten von jes nen Der Mordeln gerabe das Eegentbeil : Denn fie beftetien in Bellen von ber untern uno Blafen yon bet obern Seite: auth finb bie lappen ihres Şutes auf ber Unterfeite ni由t meiß fondern bräun. lich, uno roirtlid) nod bunEler, als auf ber DGern.

Die Zubereitung if mit ben Mordeln einer. (ey) bie Büte mittelmäffig; bie Errfheinungszeit ber Detober uno ber Stanbort gemifate laub. wälber, bie lange grünen, allwo er unter Bes büiden aus ber fimmargen 2 alderbe hervor fproft. 
XXIX. Die gemeine Mordel. Morchella esculenta, $P$.

ธ. 23udłspräp. EE. นnঠ 24b. Tab. EE.

Sie gemeine Morchel beifit in Defferreith Maus racte, auch Doppelte Maurache, fonft Mordie,

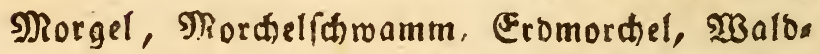
mordel, Sartenmorhel, Spismordel, solläno. Morilje, Dän. Morkler, Sđirod. Murkla, Engl. Morel, Fran\}. Morille, Stal. Spugnola,

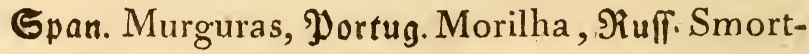

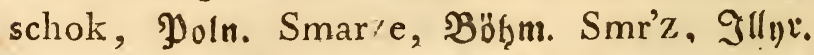
Smortschok, Rrain. Mauroche, Ungr. Kutsma. gomba, \{ett. Kehwupuppas, Effthn. Lemna nissed, u. .. w.

In meinem größ̈̈eren Sđwammwerfe (T. I. p. 67) babe i由 bie gegenwärtige Mordbelart für 

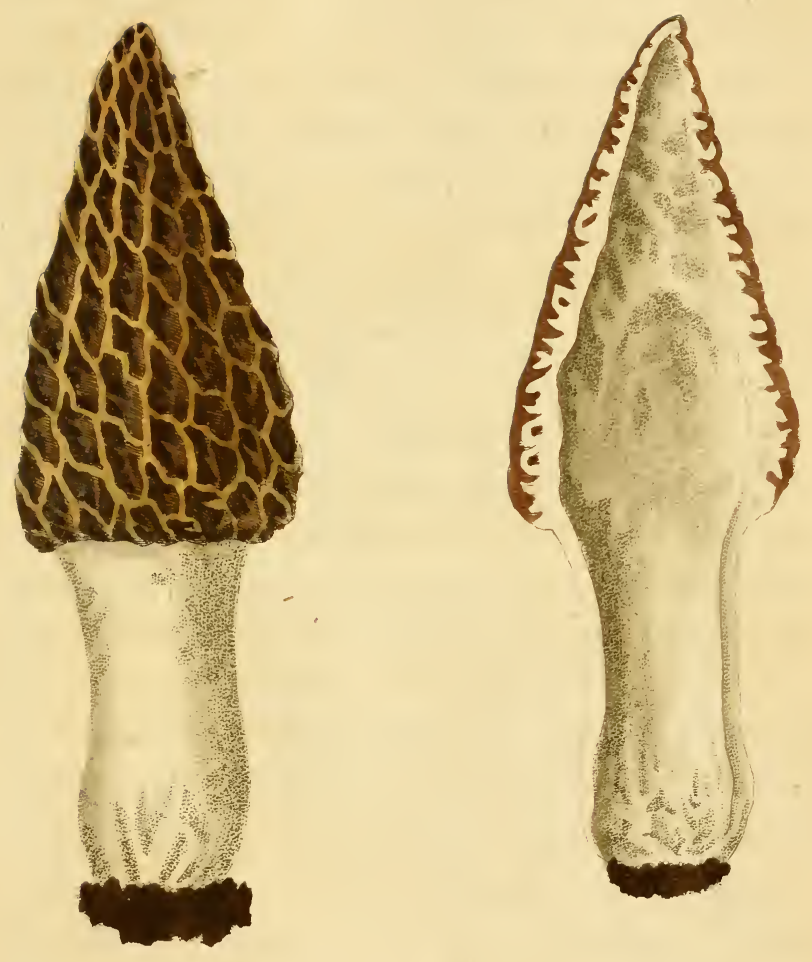

- DX ie qemeine e thorchet.

- Norchellor escudenda. Ders. 

cine befonbere Species erElärt und if̨r ben $\mathfrak{R}$ af̧a men : Morchella continua bengelegt, weil गुers foon in feincr Synopsis fungorum auborüclich fagt, Daf bie Morch. esculenta einen vollen Etrun habe, Da bod die mir befannte abjus handelnbe Sordial allezeit in StrunEe ganz

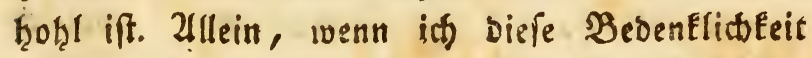
recht exnfthaft in B̧etractung nelgme: fo fdoeint es nit nun, baf entweder Ylerfoon fich geirre

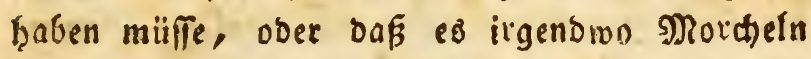
mit vollen Strünf́en geben möge; vie aber im Uebrizan von ber gemeinen Miorthel in gar nitta verffieben find. Denn bie eben von Perfoon augeführte Synonimie zeugt flar, onj er eben Denfelben Stimamm gemeint habe, notfen id a. a. D. vorgeftellet und befffrieben habe.

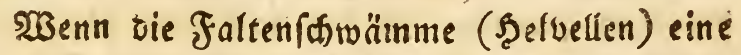
flarfe Berwandthaft zu ben Mortigelin uno Đes zizen vertathen: fo gibt uns hier bie Bietrach= tung eine Unalogie ber Morweln mit ben Udbers fđouammen (Merulius) zi enträtḩfeln. Man Dilrfte fid nur ben Oipfel einer Niorael, bet obnebem ziemlia Runfelloz, ja zumeilen woḩ gar burdfochen ift, als expeitert, geebnet uno Rippenlos denfen, fo fehlet nicht metre gar Bieles, onßj wix unz einen Merulius clavatus. vorgemahlt hätten, uno bie fogenannten Meru= lii resupinati, wie o. 
ens, wenn gleid ighre Seffalt gang verfhieben ift, haben bow faft eben folde Bellen, wie bie Mordieln. 2lber vor allen übrigen mú Merulius Pezizoides (aub ber Befdreibung зu urtḩeilen!) ben Morchellen fich nähern. Uno was fino woht biefe Exhabentgeiten um bie zellen herum anbers, als ein 2loergeflectit, obgleicts eE fich bey den Miordiellen etras regelmäffiger uns bäufiger als bey) ben 2loerforrämmen burd. Ereujet? I由 fübre viefe und äţnlithe Bemers Eungen nidbt ofne lliracte an. Misine 2lbfictis hiebey gehtet bahin, ben sefer auf bie zahllofen Uebergänge in ben Familien ber Garämme aufmerffam zu maden; unb iţn biemit ju ges miffen Betrauptungen b: rzubereiten, bie id in Der Folge ubber bie Ratur ber Sdrö̈mme vore zutragen gebenfe, unb bie aufferdem benm erfter Iuffitoflen parabor fheinen bürften. So aber barf ich es wagen, vorläufig als cine Erfaţs rungefache ben Sas aufzuftellen: ba reits angen of menen Sattungen ber Sकrömme nidt biel beffer als bie Genera im Mineralreict fino, uno

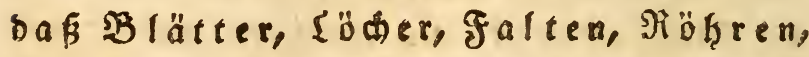
Stadeln, গiunjeln, Rn̈̈pfe u. D. gl. teine fo fanohaften Drgane find, a u

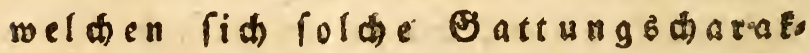
tere entlefren liésen; es räre allet. 
bings mägli angenolimenen $S$ drammgattungen, cine und biefelbe Ulteininal als Agaricus, ein andermal als Merulius, ein andermal als Daedalea, ober als Sistotrema, erffeinen bürfte; oaß alfo

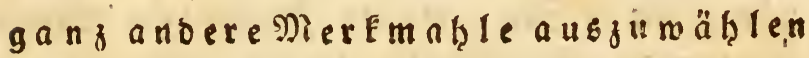
feyen, wenn man unter ben $S_{\text {dröm }}$ men eben fo fefte uno fidere os at tune gen einzuführen wünfhet, als wir bes renbereits in bem Sebiethe der गुbुo noganen a uf $z$ useifen haben.

Die gemeine Morthel hat einen unregelmäffigs

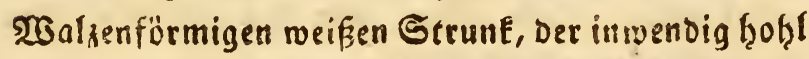
und oberbalb mit einem Şute geftönt ift, welcher der Regel naw eben fo lang als ber Strunt iff, uno ber mit feinem Ranbe auf beln obern Ende Des Strunles rings um of̣ne Brwifá)enraum felt fitct. Ex ift bon Tarbe graubraun, uno theilt fith an reiner ganzen Dberiä(f) in nidis ganz regelmäfige zieraliá) großje Zzillen, beren erhas

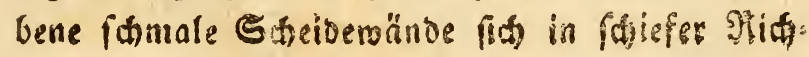
tung ructifreuzen.

Sie erfheint ben ung za Unfang oge Mane monaths in Saubwäloern ober aud) in Doftgär. ten, wenn ber Rafenbuden einige Jabre vorher mit 2ffole Berberlotie uno Baumlaube geoingt worben, who reenn die lage fo befdaffen iff, 


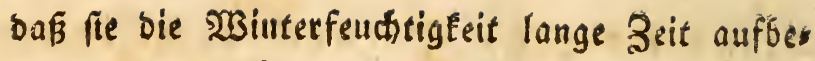
halten Eann. Man hat vor Beiten, ju grefenen

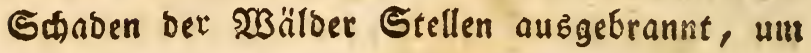
Den Wachstf̧um biefer Mordineln zu bigünftigen

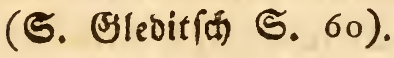

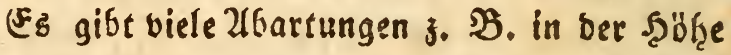
von 1 bis 3 Foll, in ber Farbe vom Eraus Graunen ins Belbbraune, in der Jigur mit eio nem (pişigen ober frumpfen Şute, weld) zureilen länger oder Eürzer ole Der Gtrune iff. Nan geniefft if̧n als Semüle man fámort ihn in Butter, mit cinem 3ufats von Zucter und Bein, man füllt ihn mit fricaffirten ses ferbiecten. 2luch pflegt man iţn getrodfnet für ben Insinter aufzubetzalten. 



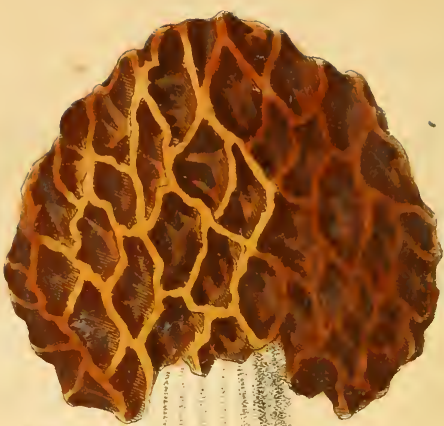

¿.

I

Der Bativamorchel.

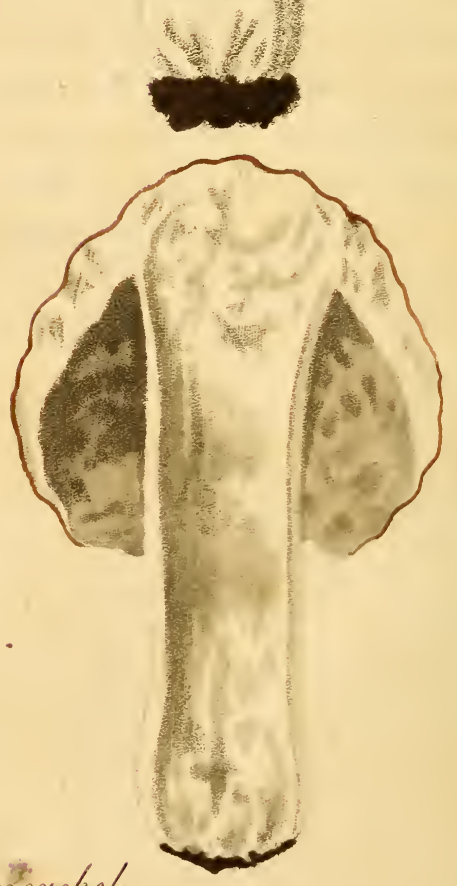

- Horchella patula. Pess. 


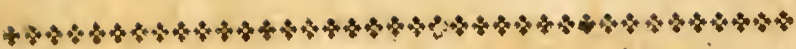

XXX. Die Saftaromordel. Morchella patula. $\mathrm{P}$.

ธ. 23adjaprăp. FF, 2(bbilo. Tab, FF.

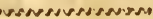

$\mathscr{A}$ verftiebene andere Zrten bon Morcteln ange. troffen, als \%. crassipes u. o. gl. Allein, ba ith diefe niemahle in ihrer Beburtbfïtte feloft gefunden hate, und mid baber bon ibrer Etättigfeit nicht gen Ennnte, fo mage id es nidht, berfelben hierorte als eigener einţeimilober Irten Erroüh, nung zu mactent.

Die Baftartmordiel habe ich bfters in sen Donauinfeln beobaditet. Sis iff ber vorigen felge äbnlich, uno unterfiteibet fich von if̧r vornälms

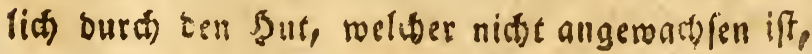


fonbern ringe um fren uber ben Gtrune herab. hängt. Seine Beflen fino etraas größ̈er uno roe: niger regelmäfrig, als bey ber gemeinen Nor, कel. Uebrigens varirt biefe Mordel eben fo wie bie gemeine.

Erfoteinunggzeit und Sebraud fino mit ber

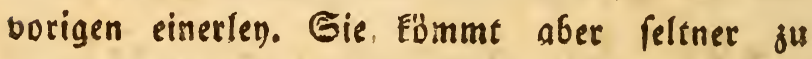
MarEte, unb ift überhaupt nicht fo bäufig zu finden. Uub pflegt fie if̧ren Stanoort, wie alle Y) fanjen Der überformemmten Begenben, offers zu bermechleln. Sie geţe leicht in Fäu!ung ïber, wenn fie einmaht zu ftäuben angefangen, uno alsbann ift if̨r Breftane unerträglid. Man muf́ bie Mlorcheln pflüfen, ba fie now jung finb: benn bie ausgerwahenen fino meiftens fápn weich, von Maben bewohnt unb eben befinegen giftig. 


York Botanical Garden Library

QK617.T7 C.2

Trattinnick, Leopol/Die essbaren schwamm

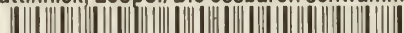

|||||||||||||||||||||||||||||||||||||||||||||||||||||||

3 5185000019941 


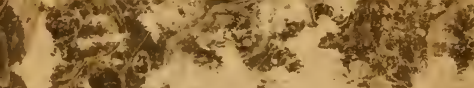

1.

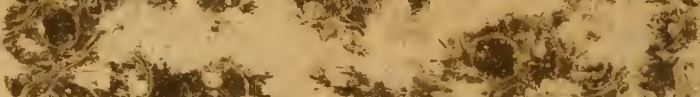

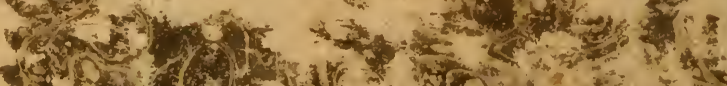

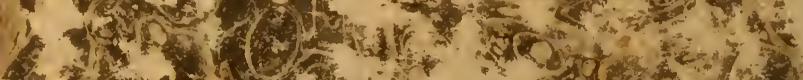

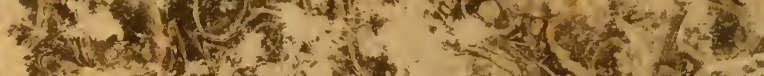

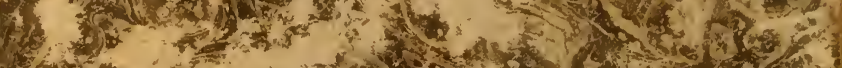

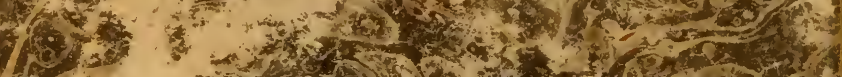

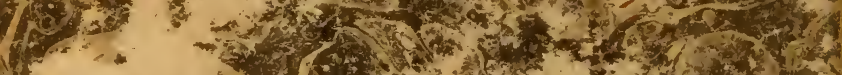
$1 \%$

(i)

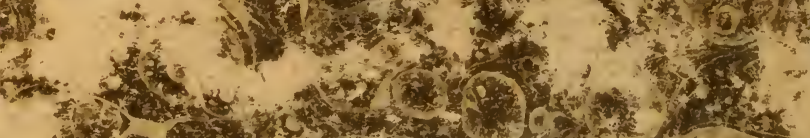

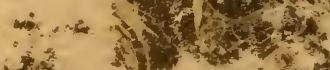

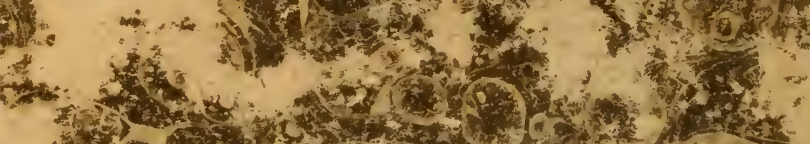

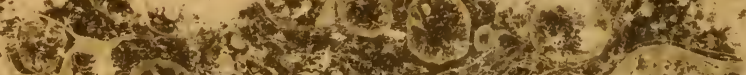

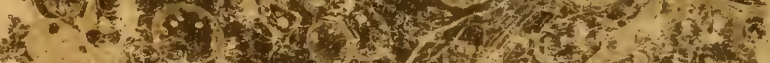

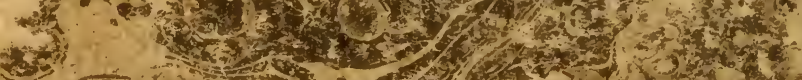

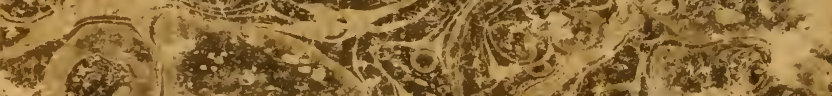

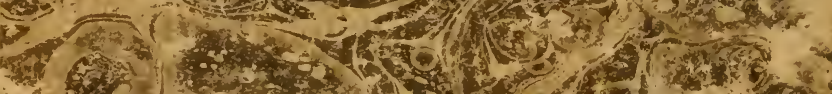

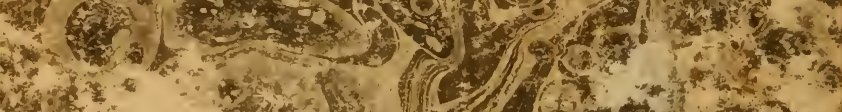

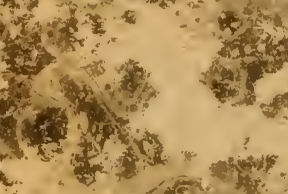
1. 130

- $+3 \%$

$+4$

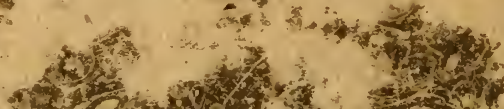

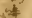

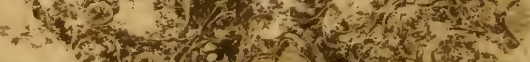

3 Nhor

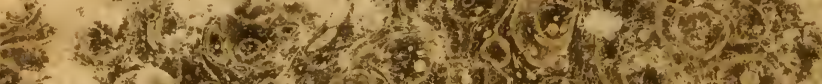

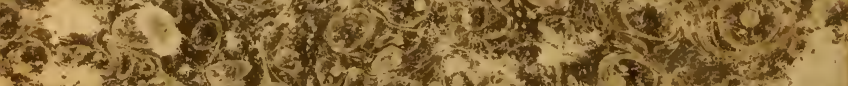

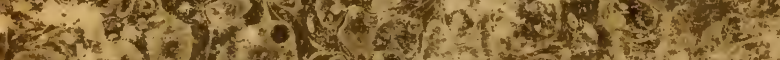
(2)

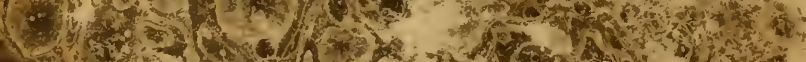

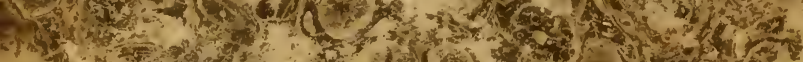

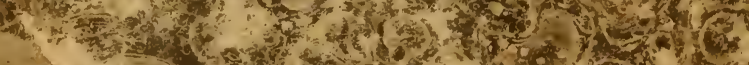

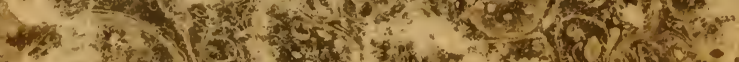

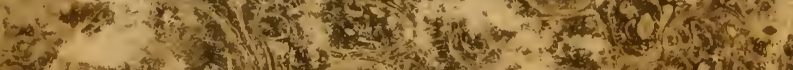

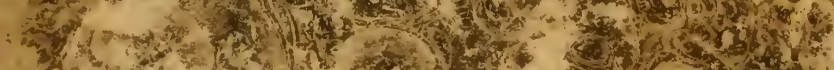

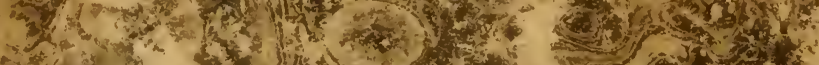
t.

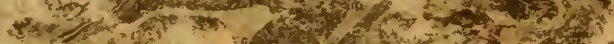

